\title{
MicroRNAs and epigenetics in chemical carcinogenesis : an integrative toxicogenomics-based approach
}

Citation for published version (APA):

Rieswijk, L. (2016). MicroRNAs and epigenetics in chemical carcinogenesis : an integrative toxicogenomics-based approach. [Doctoral Thesis, Maastricht University]. Uitgeverij BOXPress. https://doi.org/10.26481/dis.20160120lr

Document status and date:

Published: 01/01/2016

DOI:

10.26481/dis.20160120Ir

Document Version:

Publisher's PDF, also known as Version of record

\section{Please check the document version of this publication:}

- A submitted manuscript is the version of the article upon submission and before peer-review. There can be important differences between the submitted version and the official published version of record. People interested in the research are advised to contact the author for the final version of the publication, or visit the DOI to the publisher's website.

- The final author version and the galley proof are versions of the publication after peer review.

- The final published version features the final layout of the paper including the volume, issue and page numbers.

Link to publication

\footnotetext{
General rights rights.

- You may freely distribute the URL identifying the publication in the public portal. please follow below link for the End User Agreement:

www.umlib.nl/taverne-license

Take down policy

If you believe that this document breaches copyright please contact us at:

repository@maastrichtuniversity.nl

providing details and we will investigate your claim.
}

Copyright and moral rights for the publications made accessible in the public portal are retained by the authors and/or other copyright owners and it is a condition of accessing publications that users recognise and abide by the legal requirements associated with these

- Users may download and print one copy of any publication from the public portal for the purpose of private study or research.

- You may not further distribute the material or use it for any profit-making activity or commercial gain

If the publication is distributed under the terms of Article $25 \mathrm{fa}$ of the Dutch Copyright Act, indicated by the "Taverne" license above, 


\section{MicroRNAs and epigenetics in chemical carcinogenesis}

An integrative toxicogenomics-based approach 
MicroRNAs and epigenetics in chemical carcinogenesis - An integrative toxicogenomics-based approach

Thesis, Maastricht University, Maastricht, the Netherlands

ISBN: 978-94-6295-406-9

(C) Copyright 2016, Linda Rieswijk, Maastricht, the Netherlands

Cover design and layout: Linda Rieswijk

Printed by: Proefschriftmaken.nl || Uitgeverij BOXPress

All rights reserved. No part of this thesis may be reproduced or transmitted in any form or by any means, electronic or mechanical, including photocopying, recording or any information storage or retrieval system without permission in writing from the author, or, when appropriate, from the publisher of the publications. 


\section{MicroRNAs and epigenetics in chemical carcinogenesis}

An integrative toxicogenomics-based approach

\section{PROEFSCHRIFT}

Ter verkrijging van de graad van doctor aan de Universiteit Maastricht, op gezag van de Rector Magnificus, Prof. Dr. L.L.G. Soete, volgens het besluit van het College van Decanen, in het openbaar te verdedigen

op woensdag 20 januari 2016 om 12:00 uur

door

\section{Linda Rieswijk}

Geboren te Amsterdam op 7 januari 1986 


\section{Promotor}

Prof. dr. J.C.S. Kleinjans

\section{Copromotor}

Dr. S.G.J. van Breda

\section{Beoordelingscommissie}

Prof. dr. E.C.M. Mariman (voorzitter)

Prof. dr. H. van Loveren

Dr. H. Vrieling (Leids Universitair Medisch Centrum)

Prof. dr. B. van de Water (Universiteit Leiden)

The studies presented in this thesis were performed at the School for Oncology and Developmental Biology (GROW), accredited by the Royal Netherlands Academy of Arts and Sciences (KNAW). The research described in this thesis was funded by the Netherlands Genomics Initiative (NGI), the Netherlands Organization for Scientific Research (NWO), the Netherlands Toxicogenomic Center (NTC) [grant number 05006-510], and the FP7 project DETECTIVE [grant number 266838].

Financial support by Exiqon for the publication of this thesis is gratefully acknowledged. 


\section{Contents}

Chapter 1

General Introduction

Chapter 2

Characterization of cisplatin-induced transcriptomics responses in primary mouse hepatocytes, HepG2 cells and mouse embryonic stem cells shows conservation of regulating transcription factor networks.

\section{Chapter 3}

Evaluating microRNA profiles reveals discriminative responses following genotoxic or non-genotoxic carcinogen exposure in primary mouse hepatocytes.

\section{Chapter 4}

Improving the predictability of in vivo genotoxicity and carcinogenicity by exploiting microRNA and mRNA profiles generated in vitro from exposed primary mouse hepatocytes.

\section{Chapter 5}

Aflatoxin B1 exposure induces epigenetic mechanisms in primary human hepatocytes revealing novel biological processes associated with hepatocellular carcinoma.

\section{Chapter 6}

Persistent microRNA-gene networks in primary human hepatocytes upon withdrawal of aflatoxin B1 exposure are related to hepatocellular carcinoma.

\section{Chapter 7}

Aflatoxin B1 induces persistent epigenomic effects in primary human hepatocytes associated with hepatocellular carcinoma.

\section{Chapter 8}

Summary and General discussion

\section{Addendum}

I. Samenvatting en Algemene discussie

II. Valorization

III. Dankwoord 
C

00

0

O

0

O

0

Chapter 1

$00=0$

O

General Introduction

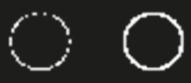

O

O

○

00

() 0

○

$\bigcirc$

O<smiles>O</smiles>

00

○

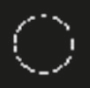

0

()

0

0

C

○

$\mathrm{O}$

00

00

(j)

000

O

0

() 


\section{General Introduction}

\section{Cancer incidence worldwide}

According to the World Health Organization (WHO), cancer is the leading cause of death worldwide accounting for 8.2 million deaths in 2012. In that same year approximately 14 million new cases of cancer were being diagnosed and it is expected to increase with $70 \%$ in the next twenty years ${ }^{1,2}$. With 782,000 of new cases per year in 2012, liver cancer (e.g. hepatocellular carcinoma (HCC) ) is, worldwide, not the most common type of cancer ${ }^{2}$. It is, however, due to its poor prognosis, the second most lethal type of cancer, and was in 2012 responsible for more than 745,000 deaths ${ }^{2}$. Many of these new cases and deaths occur in Africa, Asia and Central and South America ${ }^{1}$. In Asian countries, HCC is mainly caused by high rates of hepatitis B and C, as well as widespread alcohol use. In Western Africa, the most contributing factor is exposure to high levels of aflatoxins through the food chain ${ }^{2}$. The onset of HCC is a multistage process characterized by the progression from a pre-cancerous state to the occurrence of malignant tumors. In most HCC cases this is the result of genetic aspects on one hand and external factors (e.g. unhealthy diet or physical inactivity) or exposure to chemical agents on the other.

\section{Environmental chemicals and cancer prevalence}

External or environmental factors which may contribute to the initiation of HCC are physical carcinogens (e.g. by ultraviolet radiation), chemical carcinogens (e.g. by the food contaminant aflatoxin B1) or biological carcinogens (e.g. by hepatitis B or C viruses) ${ }^{1,3}$. Chemical carcinogens are traditionally divided, based on their mode of action (MOA), into genotoxic (GTX) and non-genotoxic (NGTX) carcinogens. GTX carcinogens (e.g. cisplatin, benzo[a]pyrene and aflatoxin B1) initiate cancer directly by damaging or reacting with the DNA thereby causing mutations ultimately leading to cancer-related events. The mechanisms by which NGTX carcinogens (e.g. 2,3,7,8-tetrachloordibenzo-p-dioxine or WY-14643) promote cancer are fairly diverse e.g. via oxidative stress initiated by binding to the transcription factors arylhydrocarbon receptor (AhR) or peroxisome proliferator-activated receptor alpha (PPAR- $\alpha)^{4,5}$. By avoiding and controlling exposure to these external agents (e.g. occupational hazards) approximately $30 \%$ of all new cases may be prevented ${ }^{3}$. Proper carcinogenicity testing and risk assessment, of new and existing chemicals, is required in order to effectively avoid and control human exposure to carcinogenic agents.

\section{Chemical carcinogenicity testing}

\section{Legislation on carcinogenicity testing}

In order to reduce the exposure to carcinogens which could initiate HCC, preliminary carcinogenicity testing of toxic agents (e.g. chemicals, drugs or nutrients) is necessary. Testing of new chemicals, drugs or nutrients for their carcinogenic potential should nowadays occur according to so called OECD Test Guidelines ${ }^{6}$. The Organization for Economic Co-operation and Development (OECD), a worldwide governmental organization, provides these internationally agreed test methods to achieve harmonization between governments, industry and independent laboratories. Within the European Union (EU), the regulatory European Chemical Association (ECHA) is the responsible organ for the registration, evaluation, authorization and restriction processes of chemical substances under the REACH (Registration, Evaluation, Authorization and restrictions of Chemicals) regulation to ensure 
consistency in the management of chemicals across the EU ${ }^{7}$. It encompasses the European legislation on chemicals with the purpose of improving protection of human health and the environment from the risks that can be posed by chemicals, while also enhancing the competitiveness of the EU chemicals industry. REACH recommends that chemicals which are marketed at more than one ton per year need to be registered by presenting an extensive dossier to ECHA.

In addition, it also encourages the development of alternative methods for the hazard assessment of substances in order to reduce the number of tests on animals. Similarly, the European Food Safety Authority (EFSA) ${ }^{8}$ is the organization in charge assessing toxicity or carcinogenicity risks from long-term consumption of nutrients or whole food by humans. In parallel, the European Medicines Agency (EMA) 9 exists, which performs equivalent testing for drugs, often in collaboration with the Food and Drug Administration (FDA) ${ }^{10}$ situated in the United States. Both EFSA as well as EMA/FDA have their own guidance documents for carcinogenicity testing but these are, like the work done by ECHA, also in agreement with the OECD guidelines.

Positive results for toxicity and carcinogenicity testing might prevent a chemical, drug or nutrient from entering the clinic or public market ${ }^{11}$. In case of a positive result, additional in vitro as well as in vivo tests are required, to determine whether the potential carcinogenic risk is genuine ${ }^{11}$. These, sometimes unnecessary, additional tests require a lot of time and money. Furthermore, clinical trials which are essential for several phases of drug development, need to be put on hold. More importantly, in case of a genuine carcinogenic hazard, new candidate chemicals, drugs or nutrients need to be developed ${ }^{11}$.

\section{Battery of in vitro genotoxicity assays}

The initial genotoxicity assays are performed by means of short-term in vitro systems which are applied individually or in a battery. The tests which are most frequently used within in vitro genotoxicity testing to predict in vivo carcinogenicity are: the bacterial Ames mutagenicity assay ${ }^{12}$, mouse lymphoma mutagen assay (MLA) ${ }^{13}$, micronucleus (MN) ${ }^{14}$ and chromosomal aberrations (CA) 15 test. The sensitivity (correctness of positive results) of these assays is relatively high when applied individually and increases when assay results are combined ${ }^{16}$. The specificity (correctness of negative results) is however fairly low and thus these in vitro based alternative genotoxicity tests are frequently associated with a high faIse positive rate ${ }^{16-18}$. A false positive result in this case indicates that a compound is genotoxic in the in vitro setting but not in the in vivo situation. This is quite problematic since in case of a positive result, further in vivo confirmation in the carcinogenicity-based 2 -year rodent bioassay is required. In order to limit the number of animal-based experiments, for false positive chemicals, it is decided on a case-by-case basis whether this is really necessary. In addition to the high degree of false positives generated with these in vitro genotoxicity tests, another discrepancy arises which is the inability to detect non-genotoxic carcinogens. Better in vitro models for accurately predicting genotoxicity and (non-genotoxic) carcinogenicity are thus required. The European Union Reference Laboratory for Alternatives to Animal Testing (EURL-ECVAM) 19 is the responsible organ within the EU to validate these alternative non-animal based test systems.

\section{Lifetime cancer bioassays}

Traditionally chemical carcinogens are classified by means of a lifetime rodent bioassay. This 2year bioassay is referred to as "lifetime" cancer bioassays as they are intended to simulate 
lifetime human exposure from a chemical compound ( $\sim 70$ years $)^{20}$. The concept of these assays is to expose the rodents to fairly high doses of a test compound, and then by applying straight-line extrapolation in combination with uncertainty factors, acceptable exposure levels in the human population are estimated. These assays are however, in general, fairly cost and time ineffective since they require the use of a high number of animals. In addition to all this, the cancer bioassay is limited by its relatively low predictive value of the results coming mainly from rodents (e.g. mice, rats and hamsters) ${ }^{21}$. The sensitivity of the lifetime rodent assay for prediction of human carcinogens is only $50 \% 21$.

\section{Development of alternative test systems for human risk assessment}

As already stated by Russell and Burch in 195922 as being the 3Rs, alternative toxicological tests are methods which should focus on the reduction of the numbers of animals used for a particular endpoint, refining a test by decreasing the pain and suffering endured by test animals, or replacing the animal test by not using any animals at all. Furthermore, development of alternative assays should be focused on generating a more mechanistic biological understanding of the nature of toxicity at the molecular, cellular, and organ level ${ }^{23}$. This might also help in understanding the results in a better way when focusing on extrapolation to humans, thereby improving risk assessment. Robust human-based alternative assays would avoid the need for interspecies extrapolation ${ }^{23}$. Nowadays the paradigm in human risk assessment has switched towards a more systems biology approach. Thereby applying pathway-based analyses to characterize the processes by which toxic agents induce adverse health effects. These pathways are described as adverse outcome pathways (AOP) ${ }^{23}$. Pathwaybased approaches will result in a better interpretation of high-throughput molecular assays, and will possibly advance intensely our understanding of the mechanisms or modes of action (MOA) underlying genotoxicity and carcinogenicity.

\section{Frequently applied in vitro liver models for genotoxicity and carcinogenicity testing}

Besides the fact that current in vitro models, for genotoxicity and carcinogenicity, are characterized by a high false positive rate and are not applicable for the detection of nongenotoxic carcinogens, they also are not representative for target organ-specific carcinogenesis. The liver, where biotransformation of many xenobiotics takes place, is the main target organ of many carcinogens. Therefore appropriate in vitro liver models should be identified which could be representative for in vivo HCC development. Biotransformation is the process in which xenobiotics are metabolically converted in the body by so called cytochrome P450 enzymes 24,25 . All xenobiotics which are taken up by the intestine are transported to the liver via the vena porta. Inhaled xenobiotics will also end up in the liver via the general circulation 24,25. Biotransformation mainly occurs in organisms in order to protect them from toxicity by preventing accumulation of harmful substances in the body. Reactive intermediate metabolites may however be formed via metabolic activation which may potentially be harmful. In the phase I reaction, hepatic cytochrome P450 enzymes initiate the conversion of xenobiotic compounds via an oxidation, reduction or hydrolysis reaction in order to assign a more hydrophilic character to the parent compound 24,25 to encourage excretion. However, often these conversion reactions result in an even more reactive byproduct. Many xenobiotics are subsequently further metabolized via the phase II reaction. Converted, intermediate metabolites that do not pass to the phase II reaction can bind with and damage cellular structures such as the DNA. The primary goal of phase II conjugation is generating more water-soluble active substrates from the phase I reaction to more inactive products which may 
be excreted or further metabolized in phase III reactions. In phase III, further modifications take place followed by excretion of metabolites from the body via the kidneys or the bile 24,25 .

\section{Primary human hepatocytes}

The best in vitro alternative test system for human risk assessment consists of primary human hepatocytes $\left(\mathrm{PHH}{ }^{26-28}\right.$. PHH, cultured in vitro between collagen sandwich layers, present an in vivo-like configuration and therefore also maintain sufficient levels of cytochrome P450 enzymes needed for biotransformation of xenobiotic compounds $26-28$. Fresh human liver samples from patients are scarce; however cryopreserved hepatocytes might be used as an alternative ${ }^{29}$. The culturing of cryopreserved PHH is however laborious and purchasing is quite costly. In addition, inter-individual differences need to be taken into account when analyzing toxicological effects used for human risk assessment.

\section{Primary mouse hepatocytes}

As an alternative for $\mathrm{PHH}$, primary mouse hepatocytes (PMH) might be used, thereby also considering their connection with the lifetime bioassays which are frequently performed in intact mice. As is the case for $\mathrm{PHH}$, also $\mathrm{PMH}$ are metabolically competent and show an in vivo-like configuration when cultured between sandwich layers of collagen. Another advantage of PMH over PHH is that they are easily available and, in general, show lesser inter-individual differences. In addition, PMH also are more preferred over primary hepatocytes originating from rats since they are metabolically more stable ${ }^{30}$. Although animals still need to be sacrificed for in vitro culturing of PMH in toxicological risk assessment, they require considerably fewer numbers of mice. With the liver of only one mouse, multiple chemical compounds might be tested for their toxic potential ${ }^{31}$, making this a good candidate for an alternative in vitro test system. The extrapolation of in vitro data originating from mice, to the in vivo human situation might be however quite complex, and might require extensive comparison with human in vivo data.

\section{Immortalized cell lines}

Two examples of immortalized cell lines which are frequently used within genotoxicity and carcinogenicity testing are the commercially available human hepatocellular carcinoma cell line (HepG2) and the cell line HepaRG. HepaRG cells are terminally differentiated hepatic cells derived from a human hepatic progenitor cell line ${ }^{32}$. In this way HepaRG cells strongly resembles primary human hepatocytes mainly due to their high cytochrome P450 enzyme activity and complete expression of all nuclear receptors. Jennen et al. ${ }^{32}$ compared HepG2, HepaRG and PHH by whole genome gene expression analysis and concluded that HepaRG is a more suitable in vitro liver model for biological understanding of chemically-induced toxic effects, while HepG2 is a more useful in vitro liver model for toxicogenomics-based classification studies. The human cell line HepG2 originates from a 15-year old Caucasian male who suffered from well differentiated HCC and may be purchased via the non-profit American Type Culture Collection (ATCC) organization. HepG2 is a well-established and easy to culture in vitro liver model which has been widely accepted as an alternative test system. Due to relatively lower levels of specific cytochrome P450 enzymes HepG2 cells are less metabolically competent in comparison to primary hepatocytes ${ }^{33}$. Nevertheless a lot of toxicological studies are still performed with HepG2 cells in which metabolic activation leading to toxic effects was observed ${ }^{32,34-41}$. 
Embryonic stem cells

Another alternative in vitro test system which is frequently applied within toxicological studies is presented by embryonic stem cells (ESCs). In general, the advantage of ESCs is that they are able to undergo self-renewal and due to their pluripotent characteristic, differentiate into any type of specialized cells (e.g. hepatocytes) ${ }^{42-45}$. Furthermore, ESCs have a normal diploid karyotype and, especially the ones from murine origin, are widely available for high-throughput assays. An additional advantage of human ESC (hESCs) for human risk assessment is that inter-species extrapolation is not necessary ${ }^{46}$. Due to some ethical constraints however, hESCs specifically, are not largely available and therefore mouse ESC (mESCs) might be used instead within toxicological studies ${ }^{44,47}$. Another disadvantage of ESC is that they are not as metabolically competent as primary hepatocytes and therefore their use within toxicological studies investigating xenobiotics which require metabolic activation by cytochrome P450 enzymes might be questioned. The combined use of ESC with more metabolically competent cells has already been proposed as a better system for improving the detection of toxicants which require biotransformation ${ }^{48}$.

\section{Systems biology based approaches within the field of toxicogenomics}

Within the field of toxicogenomics, several high-throughput omics-based platforms may be applied to study the genome, transcriptome, proteome, metabolome and even the microRNAome and epigenome simultaneously within in vitro liver models. By the application of toxicogenomics, insight might be obtained with regards to mechanisms underlying genotoxicity and (non-genotoxic) carcinogenicity ${ }^{49}$. A better understanding of these toxic mechanisms, may lead to an improved and more accurate and sensitive prediction analysis of toxicity for the human in vivo situation than is currently achieved with the battery of in vitro genotoxicity assays ${ }^{49}$.

Studying thousands of genes and their expression and regulation allows an extensive and integrative analysis of the potential mechanisms of toxicity of chemical compounds. Furthermore, toxicogenomics may offer understanding of the gene environment interactions and the response of biologic pathways and networks to chemical stresses. These types of analyses would not be possible with the outcomes of solely traditional in vitro or in vivo toxicity assays ${ }^{49}$.

\section{Studying the transcriptome for human risk assessment}

Transcriptomics encompasses the monitoring of gene expression or $\mathrm{mRNA}$ profiles of thousands of genes simultaneously, within a cell or tissue ${ }^{50}$. Over the last decade, gene expression changes have usually been measured by means of robust microarrays, however, nowadays there is a shift towards applying next generation RNA sequencing approaches. A majority of the toxicogenomics studies is, however, still conducted by using whole genome gene expression microarrays ${ }^{49}$. On a microarray, complementary DNA is present as high density probes. One single probe or a set of probes represent one particular gene. Studying the transcriptome by means of microarrays is frequently applied in the field of toxicogenomics in order to better understand the mode of action of chemicals and therefore, to more accurately predict for example chemical carcinogenicity or genotoxicity 31,32,36,51-58. 
Evaluating the microRNAome for human risk assessment

Another, relatively new field in the chemical risk assessment domain is the study of small RNA molecules via the microRNAome ${ }^{59,60}$.

\section{The function and biogenesis of microRNAs}

MicroRNAs are a dominating class of small, single-stranded, non-coding RNA molecules $(\sim 22$ nucleotides long) which are able to regulate gene expression at the post-transcriptional level ${ }^{61}$. An important aspect of microRNAs is that they are highly conserved among species, for example between humans and mice ${ }^{62}$. The biogenesis of microRNAs and their functions are depicted in Figure 1.1. MicroRNAs may be transcribed by RNA polymerase II (Pol II) from three different genomic locations; 1) as polycistronic clusters which are cleaved into multiple microRNAs, 2) as independent transcriptional units located within an intergenic region, or 3) from intronic sequences of protein-coding and non-coding transcriptional units or exonic sequences of non-coding genes ${ }^{63,64}$.

MicroRNAs are produced by two RNase III proteins, namely Drosha and Dicer, in a multistep approach ${ }^{61,63,65,66}$ (see Figure 1.1).

\section{Biogenesis of microRNAs}

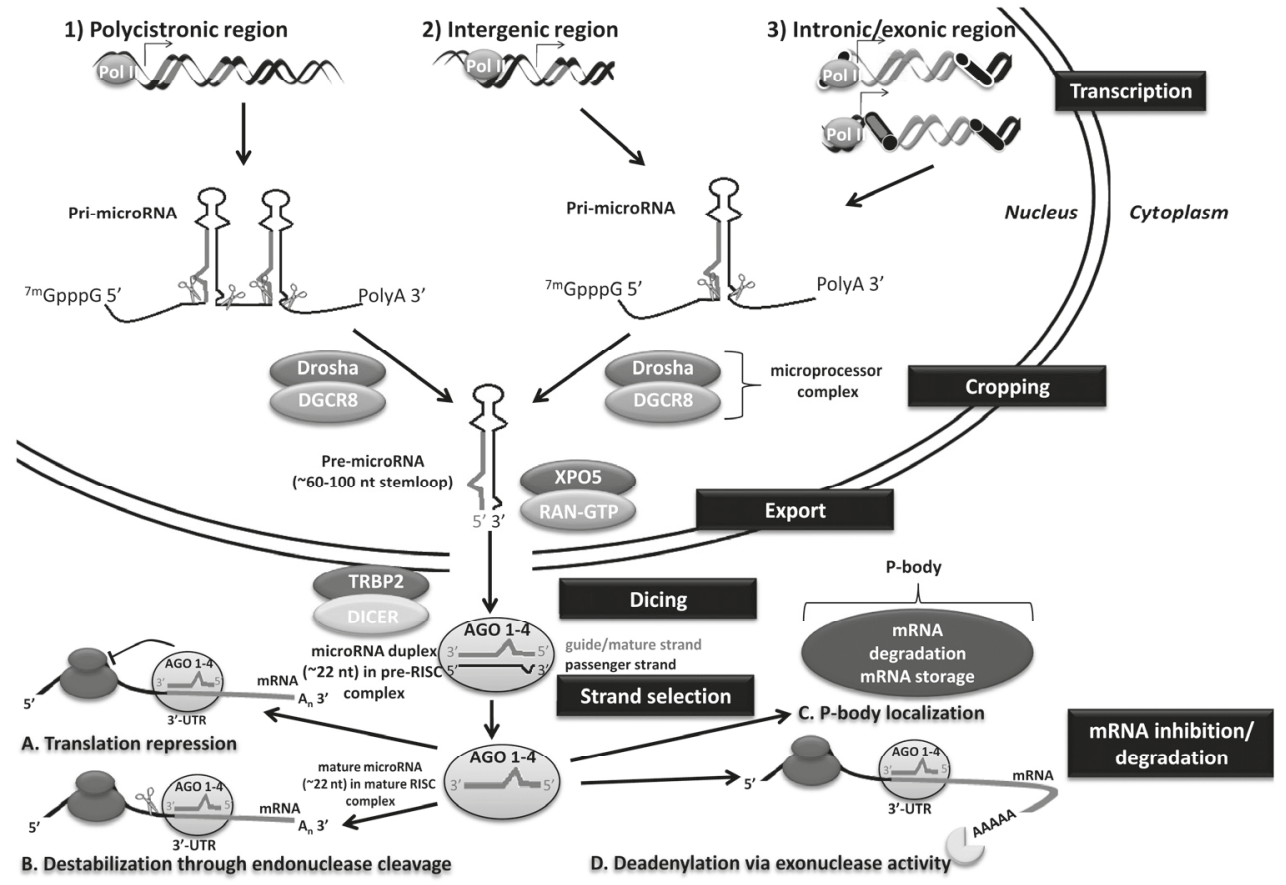

Figure 1.1: The function and biogenesis of microRNAs (adapted from Fazi et al., 2008) ${ }^{63}$ (3'=three prime end, 3'$\mathrm{UTR}=$ three prime untranslated region, 5 ' $=$ five prime end, $7 \mathrm{~m}$ GpppG $=7$-methylguanosine, AAAAA and $\mathrm{A}_{\mathrm{n}}=$ poly adenine tail, AGO 1-4 = Argonaute protein 1,2,3 and 4, DGCR8= DGCR8 microprocessor complex subunit, DICER=dicer 1, ribonuclease type III, Drosha $=$ drosha, ribonuclease type III, mRNA=messenger RNA, $\mathrm{nt}=$ nucleotides, $\mathrm{P}$-body $=$ processing body, Pol II=RNA polymerase II, TRBP2=RISC-loading complex subunit 2 , RISC $=$ RNA-induced silencing complex, XPO5=exportin 5) 
First, microRNA genes are transcribed within the nucleus as long RNA primary transcripts by RNA polymerase II (Pol II) molecules named the pri-microRNA ( 60 nucleotides long). After transcription, a 7-methylguanosine $(7 \mathrm{mGpppG})$ cap and a poly $(\mathrm{A})$ tail are attached to the primicroRNA ${ }^{63}$. Then, the pri-microRNA is cleaved by the microprocessor complex consisting of Drosha and a double-stranded RNA binding protein Pasha (also named DGCR8) to form a characteristic stem-loop or hairpin structure named the pre-microRNA ${ }^{61}$.

For maturation purposes, the pre-microRNA is then exported out of the cell nucleus via exportin 5 (XPO5) in complex with GTP-binding nuclear protein RAN.GTP to the cell cytoplasm (see Figure 1.1). In the cytoplasm, the pre-microRNA is cleaved by DICER and TRBP2 forming a RNA duplex (containing a mature or guide strand arising from 5 arm (-5p) of the hairpin and a passenger strand arising from the 3'arm of the hairpin(-3p)) $61,63,65-67$. The RNA duplex is then loaded onto one of the AGO proteins (type 1-4) thus forming the preliminary RNA-induced silencing complex (pre-RISC) ${ }^{65}$. Quickly thereafter, the passenger strand $(-3 p)$ of the RNA duplex is removed leaving the mature or guide strand $(-5 p)$ attached to the AGO protein ${ }^{61,63,65-67}$. This effector complex is called the mature-RISC complex. The less abundant passenger strand is also active in silencing, although typically less effectively than the more abundant mature strand ${ }^{65}$. MicroRNAs are highly stable once they enter RISC, because both ends are protected by AGO proteins. Therefore, for microRNA decay to occur, microRNAs may need to be unloaded first so that exonucleases can access their terminal ends $61,63,65-67$.

MicroRNAs are able to silence RNA by base pairing with its mRNA target's 3'untranslated region (UTR). The domain at the 5'end of the microRNA (mainly nucleotide position 2 to 7), also called the microRNA seed, is essential for target recognition ${ }^{61,63,65-67}$. After the formation of the mature RISC complex, gene expression may be affected in four different ways; A) via translation repression, B) via mRNA destabilization upon endonuclease cleavage, C) via localization to processing bodies (P-bodies) where mRNA degradation can take place and untranslated mRNA can be stored (recycling of $\mathrm{mRNA}$ ) or D) via mRNA deadenylation in which the 3'poly-A-tail is cut off by an exonuclease leading to mRNA degradation (see Figure 1.1) $61,63,65-67$.

Sixty percent of all protein-coding genes contain at least one conserved microRNA-binding site ${ }^{61,63,65-67}$. Therefore, microRNAs are able to target almost all protein-coding genes and are thus involved in crucial developmental and pathological processes. The biogenesis of microRNAs is tightly controlled and their expression is greatly time and tissue-specific. Dysregulation of microRNA expression is often associated with many human diseases, including cancer ${ }^{61,63,65-67}$.

\section{MicroRNA expression levels associated with hepatocellular carcinoma}

Since microRNAs play a crucial role in gene regulation, dysregulation has been observed in disease including cancer such as HCC. Several deregulated microRNAs important in cell proliferation, survival, angiogenesis and metastasis have been reported in HCC ${ }^{68-76}$. Even several polymorphisms within the sequence of the microRNAs or the 3'UTR-region of their target gene have been reported to be related with risk for developing HCC ${ }^{68}$. A number of deregulated microRNAs has been proven to serve as prognostic or diagnostic markers for HCC for example in serum ${ }^{68}$. These are for example: downregulated microRNAs microRNA122, microRNA-124, microRNA-139, microRNA-145, microRNA-199b, microRNA-22, microRNA-26, microRNA-29, microRNA-99a and let-7g associated with poor prognosis and 
survival ${ }^{68}$. Deregulated upregulated microRNAs associated with decreased prognosis and survival are; microRNA-10b, microRNA-135a, microRNA -17-5p, microRNA -155, microRNA-182, microRNA-21, microRNA-221 and microRNA-222 ${ }^{68}$. It is even suggested that delivery of microRNAs that are highly expressed in normal tissues but lost in disease cells may be used as a general strategy for microRNA replacement therapies without significant toxicity ${ }^{68}$. In addition, restoration of microRNA-122 levels ${ }^{77}$ and inhibition of microRNA-21 78 and microRNA-181b expression ${ }^{79}$, is suggested as a way to make cancer cells more sensitive to treatment with chemotherapy drugs ${ }^{68}$.

\section{MicroRN A expression levels associated with environmental carcinogen exposure}

MicroRNA dysregulation in tissue from HCC patients has well been established ${ }^{68}$; however microRNA expression changes may already occur far before the onset of HCC in healthy organisms. In this way, altered microRNA expression profiles may serve as biomarkers for early signs of HCC and may even have a predictive value in human risk assessment. A new paradigm in risks assessment has thus emerged focusing on altered microRNA expression patterns, due to environmental carcinogen exposure, occurring in healthy organisms or cells, and this could be predictive for the future development of HCC. One requirement here is that these microRNA HCC expression patterns are persistent and thus irreversible 59,60,80. The dose and the exposure time used in cell models should, in this case, be representative for long term exposure ${ }^{81}$. MicroRNA expression patterns are altered after exposure to large set of environmental carcinogens. Short term exposure might trigger a more adaptive response of the cell to the xenobiotic exposure thereby activating all kinds of microRNAs important in defensive mechanisms which are for example related with metabolic detoxification, DNA and protein repair, and apoptosis activation ${ }^{82}$. Following long term exposure, the behavior of these microRNAs may change to a more tumor-suppressive or oncogenic nature. Oncogenic microRNAs have been identified and are frequently upregulated by environmental carcinogens (e.g. cigarette smoke, silica dust, radon, 2-AAF, arsenic, TPA, benzene, toluene or ethanol): microRNA-17, microRNA-19, microRNA-21, microRNA-155, microRNA-211 and microRNA-222, which mainly target E2F1, PTEN, PDCD4, and SOCS1. Suppression of these transcripts results in increased cell proliferation, decreased apoptosis, invasion and metastasis 80 thereby initiating cancer-related events important for the development of HCC. MicroRNAs with a tumor suppressive function, e.g. let-7, microRNA-34, microRNA-125, microRNA146a, microRNA-205, microRNA-506, microRNA-22 are frequently downregulated after exposure to the before mentioned environmental carcinogens 80 thereby resulting in upregulated mRNA target genes important in many cancer-associated processes. Especially the bio-monitoring of healthy individuals or cells may therefore be very useful for detecting early events before the onset of HCC.

\section{Analyzing the epigenome within the human risk assessment domain}

In addition to the transcriptome and the microRNAome, the epigenome might also reveal interesting regulatory information which may be applied within the field of chemical risk assessment.

\section{Chromatin remodeling}

Genomic DNA is tightly packed together with histones within chromosomes present in the nucleus of the cell (see Figure 1.2). The DNA helix is wrapped around core histones to form the 'beads on a string' structure that is then folded into nucleosomes and higher-order 
chromatin ${ }^{83}$. In order for gene expression to occur, chromatin remodeling needs to take place which involves the dynamic modification of the chromatin architecture in order to open up or hide the genomic DNA for transcription ${ }^{83}$. Therefore, chromatin is not uniform with respect to gene distribution and transcriptional activity. Chromatin can be organized in two different domains; euchromatin (loose or open chromatin) and heterochromatin (tight or closed chromatin) ${ }^{83}$. The chromatin thus contains several DNA regulatory regions, not allowing them to interact with transcriptional machinery proteins and regulate gene expression ${ }^{83}$.

Chromatin modification and remodeling are involved in the maintenance of so called epigenetic gene regulation which plays a role in tissue-specific gene expression patterns and global gene silencing ${ }^{83}$.

\section{Epigenetics}

Within the field of epigenetics, the heritable changes in gene function (specifically in chromatin architecture) are studied which are not caused by changes in the primary DNA sequence. These dynamic epigenetic changes are maintained during cell division, however can change in response to environmental stimuli (e.g. nutrients and chemicals). In contrary to homogenous genetic information, epigenetic modifications are rather cell-type specific ${ }^{84}$. These epigenetic changes define the transcriptome, which determines the characteristics of each cell type. Epigenetic alterations play an important role in development and evolution but also in disease, and include: cell differentiation, post-translational histone modifications (acetylation, methylation, phosphorylation, ubiquitylation, glycosylation, ADP-ribosylation, and sumoylation) and DNA methylation (see Figure 1.2).

These all involve chromatin remodeling or modification and they can influence transcriptional activity. The epigenome is defined as the collection of all epigenetic characteristics, in parallel to the genome which represents the collection of all genetic features of an organism ${ }^{85}$.

One of the most important epigenetic marks is DNA methylation which is responsible for the regulation of genomic programming and imprinting during embryogenesis. Examples of phenomena which involve epigenetic silencing during early development include $\mathrm{X}$ chromosome inactivation and parental imprinting ${ }^{86}$. In addition, DNA methylation changes are known to occur in response to all kinds of environmental stimuli and are also altered in several diseases including cancer ${ }^{87}$. 


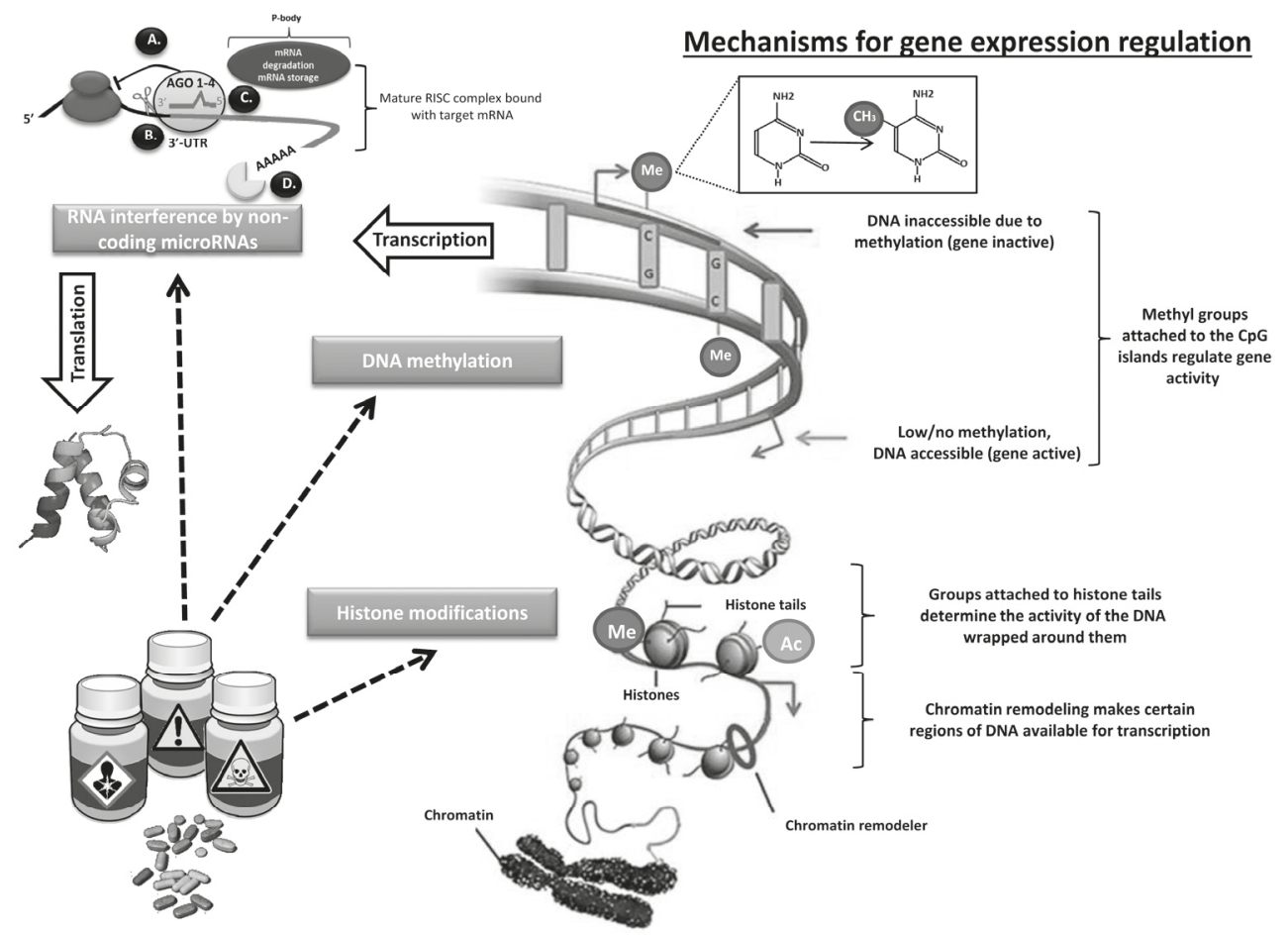

Figure 1.2: The different mechanisms for gene expression regulation (adapted from Rajender et al., $2011{ }^{88}$ ). The three main mechanisms by which the expression of genes are regulated; epigenetic mechanisms including DNA methylation and histone modifications ( $\mathrm{Me}=$ methylated cytosine or histone, $\mathrm{Ac}=$ acetylated histone, $\mathrm{CH}_{3}=$ methylated cytosine) and RNA interference by non-coding microRNAs $(A .=$ Translational repression, $B .=$ Destabilization through endonuclease cleavage, C.=P-body localization and D.=Deadenylation via exonuclease activity)

\section{$D N A$ methylation}

DNA methylation occurs at the cytosine bases that are followed by a guanine. These sites of DNA methylation are called (5'-) CpG (-3') sites. During methylation the cytosine base within the DNA is converted into 5-methylcytosine (see Figure 1.2) ${ }^{89}$. In order to maintain the correct patterns of methylation through cell division, new methyl groups are added onto freshly copied DNA, a reaction which is catalyzed by DNA methyl transferases (DMNTs) ${ }^{90}$.

In mammals, most CpG sites in DNA are methylated (90-98\%) ${ }^{91}$. This includes exons, intergenic or 'junk' DNA, and transposons. Methylation may be the default state for genes, and although the purpose of the methylation of intragenic DNA, or 'junk DNA', is unclear at this time, it may protect against expression of unwanted genes ${ }^{91}$.

There are however specific CpG-rich areas of DNA in which most CpGs are not methylated. These areas are described as CpG islands ${ }^{92,93}$. CpG islands are mainly present at the 5' end of the promoter region of about $50 \%$ of human genes, and when these islands are methylated, gene expression is usually suppressed or silenced ${ }^{92,93}$. They therefore serve as a critical switch that regulates gene expression. As a result, it is possible for a gene to be hypermethylated overall, yet have CpG islands in its promoter that are unmethylated, which results in expression of that gene. Conversely, the exons in a gene may be hypomethylated and the $\mathrm{CpG}$ islands hypermethylated, which results in a suppressed gene ${ }^{94}$. A CpG island is defined as a 
region of DNA with more than 200 base pairs, a high $\mathrm{G}$ and $\mathrm{C}$ base content and an observed/ expected ratio of $\mathrm{CpGs}$ greater than 0.6. The methylation status of $\mathrm{CpG}$ islands is believed to regulate gene transcription through the inhibition of transcription factor binding either directly or via altered histone acetylation ${ }^{95}$. The pattern of DNA methylation in CpG islands varies by tissue type and offers an explanation why genes are differentially expressed among tissues ${ }^{91}$.

The mechanism responsible for DNA methylation-associated gene silencing, seems to involve the link of specific methylated DNA binding proteins with a variety of proteins such as transcription factors, DNA methyltransferases (DNMT1, 2, 3L, 3A, and 3B), methyl-binding proteins (MeCP1 and 2, MBD1, 2, 3, and 4, and KAISO), histone-modifying enzymes (acetylases, deacetylases, methylases), chromatin remodeling factors (SWI2/SNF2, ISW1, and Mi-2) and their multi-molecular complexes ${ }^{96}$. In general, these protein/DNA interactions alter the DNA conformation, changing it to a more compact and condensed chromatin configuration (=heterochromatin), which may results in a stable inhibition of messenger RNA and protein production ${ }^{97,98}$.

Methyl groups are attached to the DNA via two different pathways, namely; the maintenance versus de novo methylation pathway. Within the early phase of mammalian development, DNA methylation patterns are largely erased and re-established just after implantation in a wave of de novo methylation 83,85,99. Two DNA methyltransferase (DMNTs) responsible for de novo methylation in embryonic stem cells and early embryos are DNMT3a and DNMT3b. After each round of replication these newly established methylation patterns are copied onto the newly synthesized DNA strand ${ }^{100}$. In contrast, genomic imprints, which are largely dependent on DNA methylation, are mostly established in germ cells and preserved throughout embryogenesis. The maintenance of DNA methylation marks, primarily by DNMT1, is found in all somatic cells while most of the de novo methylation is solely found in embryonic cell lines, germ cells, or in post implantation embryos ${ }^{100}$.

In general, a constant supply of new methyl groups is required for DNA methylation to occur. These methyl groups may be directly be provided via the food, including methionine, betaine and choline. Alternatively, mammals can make methyl groups from precursor chemicals, including folic acid ${ }^{101}$.

DNA methylation changes not only occur during development but also during certain disease states, such as cancer. Aberrant methylation of DNA, specifically global hypomethylation accompanied by promoter region-specific hypermethylation of tumor-suppressor genes, is frequently reported in tumor cells ${ }^{102}$. Global hypomethylation of the coding and the noncoding regions and demethylation of repetitive DNA sequences contribute to the development of cancer, such as HCC. This occurs mainly through chromosomal instability and increased mutations. These changes could disrupt normal cellular genes, mitotic recombination leading to loss of heterozygosity and promotion of rearrangements, aneuploidy, loss of imprinting, and up-regulation of proto-oncogenes ${ }^{103}$.

\section{DNA methylation changes associated with hepatocellular carcinoma}

Besides the association of genetic instability and mutations with HCC, also a clear relation has been established between HCC and epigenetic modifications. Alterations in DNA methylation patterns, including global hypomethylation and promoter-specific hypermethylation, are thought to be early events in hepatocarcinogenesis ${ }^{104}$. Excessive promotor hypermethylation can lead to the transcriptional silencing of genes harboring a tumor suppressive function. 
Almost two decades ago, Knudson ${ }^{105}$ developed a model, also known as the "two-hit" model, which indicates that tissue-specific epigenetic alterations play a role as one of the first hits in the development of cancer. Ten years later, Feinberg and colleagues 106 expanded this model suggesting that early epigenetic alterations are the beginning of tumor development in a three step "epigenetic progenitor model". When these epigenetic alterations occur in tissue progenitor or stem cells, this may result in either increased susceptibility to neoplastic changes or increased growth of this specific population. In the second step, genetic mutations within this cell will occur, resulting in cancer. When a cancer has occurred, the genetic and epigenetic plasticity of a cell seems to be enhanced. DNA and chromatin instability encourage the cell to further induce phenotype changes. This strongly contributes to tumor heterogeneity and the development of metastatic and drug-resistant phenotypes ${ }^{104}$. For the development of HCC, a specific mathematical model, developed by Kopp-Schneider ${ }^{107}$, suggests that epigenetic changes resulting in cancer follow a specific pattern called a 'color shift'. During this shift an entire colony of cells shifts to cancer, contrasting to the clonal model, in which cancer begins from a single cell transformation. An explanation for this phenomenon is that epigenetic changes "prime" a group of cells prior to the onset of cancer development, thereby creating predisposed "epigenetic fields" of cells ${ }^{108}$. For example hypermethylation of MGMT, an important DNA repair gene has been defined as such an epigenetic field defect ${ }^{109}$. This altered epigenetic state of the gene might induce pre-neoplastic lesions within the liver 108,109. Other epigenetic modifications were identified specifically for HCC, and these include TP53, Bcatenin, ErbB receptors, MET, hepatocyte growth factor (HGF), p16 ${ }^{\mathrm{INK} 4 \mathrm{a}}$, E-cadherin and cyclooxygenase 2 (COX-2) 110,111. Especially hypermethylation of p16 ${ }^{\text {INK4a }}$ was observed in the early phases of HCC ${ }^{112}$. In a study by Lee and colleagues ${ }^{113}$, HCC samples were paired with non-HCC samples. They identified frequently (between $80-30 \%$ of all cases) methylated genes in HCC, namely: APC, GTP1, RASSF1a, p16, COX-2 and E-cadherin. Hypermethylation of p16 and RASSF1A were also reported in similar studies by others ${ }^{111,114}$. Besides its role in the prognosis of HCC ${ }^{115}$, alterations in promoter methylation may already occur very early during carcinogenesis and can even be detected in the normal epithelia of healthy individuals.

\section{DNA methylation changes associated with environmental carcinogen exposure}

Exposure to several environmental carcinogens has already been shown to induce epigenetic modifications, specifically DNA methylation changes, in individuals and in several in vitro cell models. Examples of such chemical exposures are the genotoxic carcinogen benzo[a]pyrene $(\mathrm{BaP})$ exposure in coke-oven workers associated with global and gene-specific promoter methylation changes of p53, p16, HIC1 and IL-6 as measured in peripheral blood cells. Chromosomal instability, as was observed by micronuclei measurements, was associated with changes in DNA methylation levels ${ }^{116}$. Other examples of genotoxic carcinogens that induce methylation changes are the heavy metals arsenic, nickel, cadmium, lead and mercury whose exposure leads to global hypomethylation via S-adenosyl methionine (SAM) insufficiency which is a universal methyl donor for methyltransferases including DNA methyltransferases (DNMTs) that determine DNA methylation ${ }^{117,118}$. SAM is needed to detoxify the effects of inorganic metals entering the body. Arsenic exposure has been shown to induce dosedependent promoter hypermethylation of several tumor suppressor genes, such as p15, p16, p53, RASSF1A, PRSS3 and DAPK. Another heavy metal which induces DNA methylation changes is nickel ${ }^{119}$. Nickel has been proposed to increase chromatin condensation and trigger de novo DNA methylation of critical tumor suppressor or senescence genes. Hypermethylation of p16 and p53 was observed in mice following exposure to nickel ${ }^{117}$. Experiments with pesticides such as vinclozolin, methoxyclor and dieldrin have also identified alterations in epigenetic mechanisms ${ }^{117}$. In addition, exposure to benzene, bisphenol A, dioxin, 
RDX, diethylstilbestrol, chloroform, dichloroacetic acid and trichloroacetic acid has been proven to affect epigenetic traits as found within both in vitro as well as in vivo models ${ }^{117}$. Thus epigenetic profiling might be specific for a particular exposure and might even be predictive for the future development of a disease including HCC. One of the earliest examples to support the hypothesis, that persistent changes within the epigenome may predispose for disease, refers to individuals who prenatally suffered from famine, during the Dutch Hunger Winter between 1944 and $1945{ }^{120}$. Mainly, the DNA methylation of the imprinted gene IGF2 was reduced in comparison to equivalent of their well-nourished, same-sex siblings ${ }^{120}$. Lower methylation levels of the IGF2 gene have been associated with an increased risk of cardiovascular diseases ${ }^{120}$. This research clearly showed that early-life environmental circumstances can initiate epigenetic alterations in humans that persist throughout life 120 . These adverse environmental conditions early in development are also strongly associated with adult disease risk such as cancer. Research is however needed which focusses on the effectiveness of these changes following exposure to environmental carcinogens. Also it needs to be investigated whether these epigenetic changes accumulate following repeated or continuous exposure. Persistence of these epigenetic effects after the exposure is terminated, is hereby very crucial 117 .

\section{Integrating different regulatory layers in human risk assessment}

Until now, the majority of 'omics-related studies on environmental carcinogen exposure, has mainly focused on analyzing the transcriptome, microRNAome and epigenome separately. All of these regulatory layers, however, collaborate with each other and therefore, integrated systems biology approaches are necessary. Integration of multiple regulatory levels induced by a chemical carcinogen provides more insight in the series of events that eventually determine the expression of a gene. Pathway-based approaches may help in creating unique adverse outcome pathways (AOPs) which represent characteristic processes by which toxic agents induce adverse health effects leading to diseases such as HCC. More specifically, these integrative pathway-based analyses will contribute to a better understanding of mechanisms or MOA underlying genotoxicity and (non-genotoxic) carcinogenicity. With a better understanding of these toxicological mechanisms, predictions of chemically-induced carcinogenesis, important in human risk assessment, might be improved 23,53,59,87.

\section{Use of model compounds}

In order to further explore the role of transcriptomic, microRNA and epigenetic changes in chemical carcinogenesis, and in carcinogen-induced HCC in particular, for our studies, a group of prototypical genotoxic (GTX) and non-genotoxic (NGTX) model compounds was selected for which in vitro as well as in vivo genotoxicity and carcinogenicity data were available. This information was retrieved from in vitro and in vivo study results collected by the International Agency for Research on Cancer (IARC) ${ }^{121}$, the National Toxicology Program (NTP) ${ }^{20}$ and the Environmental Protection Agency $(\mathrm{EPA})^{122}$. For distinguishing GTX from NGTX compounds, chemicals were selected based on their genotoxicity. In order to discriminate NGTX carcinogens from non-carcinogens compound were chosen according to their nongenotoxic and carcinogenic characteristics. These prototypical model compounds are discussed in the following paragraphs. 
$\underline{\text { Genotoxic carcinogens }}$

\section{Aflatoxin B1}

Aflatoxin B1 (AFB1) is a potent hepatocarcinogenic mycotoxin produced by Aspergillus flavus and $A$. parasiticus fungi ${ }^{123}$. It is classified as a genotoxic human carcinogen ${ }^{20,121,122}$. This food contaminant causes high prevalence of HCC in developing countries ${ }^{124,125}$. AFB1 needs to be bio-transformed by cytochrome P450 enzymes in order to become active. The intermediate product of AFB1 metabolization, exo-8,9-epoxide, mainly causes irreversible DNA adducts and induces specific mutations in the p53 gene ${ }^{126-128}$.

\section{Cisplatin}

Cisplatin (cisPt) is a widely used chemotherapy agent for treating a variety of solid tumors. CisPt does not require metabolic activation for exerting its genotoxic and carcinogenic activity. CisPt is a classified as a probable human carcinogen $20,121,122$. These are mainly a consequence of the formation, especially 1,2-intrastrand $\mathrm{d}(\mathrm{GpG})$ cross-linked DNA adducts. CisPt may enter the cell by passive diffusion and facilitated active transport. Once in the cell, it transforms in a highly reactive metabolite, which may bind to the N7 of guanosine and adenine in the DNA, causing mutations and cell death ${ }^{129,130}$. CisPt treatment is characterized by a high occurrence of side effects. Especially hepatotoxicity forms a dose-limiting factor of CisPt treatment ${ }^{131}$.

\section{Benzo[a]pyrene}

Benzo[a]pyrene $(\mathrm{BaP})$ is a member of the polycyclic hydrocarbon (PAHs) family. The metabolite benzo[a]pyrene diol epoxide (BPDE), resulting from $\mathrm{BaP}$ biotransformation, is highly mutagenic and carcinogenic and $\mathrm{BaP}$ is therefore classified as a human carcinogen $20,121,122$. It is mainly found as a byproduct of incomplete combustion of organic materials (e.g. cigarette smoke and diesel exhaust) or charbroiled food ${ }^{122}$. BPDE binds with the DNA forming DNA adducts and may induce mutation in the p53 gene leading to cancer ${ }^{122}$. Furthermore $\mathrm{BaP}$ may induce an increase in one of the cytochrome P450 enzymes, CYP1A1, via binding to the aryl hydrocarbon receptor (AhR) and aryl hydrocarbon receptor nuclear translocator (ARNT) ${ }^{122}$.

\section{7,12-Dimethylbenzanthracene}

7,12-dimethylbenz[a]anthracene (DMBA) is another member of the polycyclic hydrocarbon (PAHs) family ${ }^{132}$. Although, DMBA specifically is not classified according to its carcinogenic risk in humans by IARC, NTP or EPA, PAHs are classified as human carcinogens. DMBA is an immunosuppressive agent and also a strong human carcinogen which is frequently used within cancer-related studies (mainly focused on skin cancer) 20,121,122. DMBA, similar to BaP, also requires biotransformation in order to form highly carcinogenic metabolites that initiate the formation of DNA adducts and p53 mutations ${ }^{133,134}$. Also the binding to AhR and ARNT has been observed for DMBA ${ }^{135}$.

\section{Para-Cresidine}

Para-Cresidine (paracres), a member of the aromatic amines family, is a white crystal-like compound, which functions as a chemical intermediate for the manufacturing of dyes and pigments ${ }^{136}$. It has been reported by the EPA that paracres contamination was identified in 
red dye number 40 used within gelatins, puddings, dairy products, confections and beverages 137. Exposure mainly occurs in the industrial setting via occupational dermal contact or inhalation. It has been classified as a possible human carcinogen by IARC and the NTP 20,121. Not a lot of studies have focused on the exact mechanism by which paracres induces genotoxicity as well as carcinogenicity.

\section{$\underline{\text { Genotoxic non-carcinogens }}$}

\section{Tributyltinoxide}

Tributyltin oxide (TBTO) is an effective biocidal preservative for wood, cotton textiles, paper, and paints and stains for residential homes ${ }^{122}$. TBTO is added as a so called antifouling agent in numerous formulations of marine paints. TBTO possesses an immunosuppressive function. Paints containing up to $20 \%$ tributyltin prevent the attachment and growth of barnacles, plankton, algae, and other organisms to ship hulls ${ }^{122}$. TBTO mainly induces genotoxic effects within the thymus thereby initiating apoptosis ${ }^{138,139}$. Remarkably, TBTO gave a negative result for genotoxicity within the in vitro Ames mutagenicity assay as well as the MLA assay. Positive in vitro and in vivo results were however obtained with the combination of CA/MN genotoxicity test as well as with the transgenic rodent assay (TRA) ${ }^{140}$. Though TBTO is genotoxic, results from in vivo bioassays from rats are described as questionable to humans and therefore TBTO has been assigned by the EPA as not classifiable as to human carcinogenicity ${ }^{122,136}$.

\section{Non-genotoxic carcinogens}

\section{2,3,7,8-Tetrachlorodibenzo-para-dioxin}

2,3,7,8-tetrachlorodibenzo-para-dioxin (TCDD) is a member of the polychlorinated dibenzodioxins (PCDD) or dioxin family, which is mainly formed as a byproduct of organic synthesis and incineration of organic materials ${ }^{141}$. It is the most potent human liver carcinogen of the family of PCDDs 20,121,122, which is highly biopersistent and therefore accumulates within the environment. The mode of action of TCDD is mostly initiated by its binding to the AhR receptor thereby affecting a whole spectrum of genes involved in cancer-related processes 141 .

\section{$17 \beta$-Estradiol}

$17 \beta$-estradiol (E2), is a naturally occurring primary human female sex hormone and steroid. E2 is especially important in the regulation of the estrous and menstrual female reproductive cycles ${ }^{142}$. For this reason E2 is frequently used, in combination with progestin or individually, in hormone replacement therapy in menopausal women or within infertility treatment ${ }^{142}$. Although in vivo this compound is non-genotoxic, it showed positive results for the in vitro combination of the chromosomal aberration and micronucleus test $(\mathrm{CA} / \mathrm{MN}){ }^{136}$. E2 is classified as a non-genotoxic human carcinogen by IARC and NTP 20,121 and it mainly acts via binding to two nuclear hormone receptors; estrogen receptor $\alpha(\mathrm{ER} \alpha)$ and estrogen receptor $\beta$ $(E R \beta){ }^{143}$. Activation of these receptors might enhance the expression of cancer-related genes e.g. related with cell cycle ultimately leading to cancer ${ }^{144}$. 
Di(2-ethylhexyl)phthalate (DEHP) is a manufactured chemical that is commonly added to plastics to make them more flexible. It is present in many plastics, especially polyvinyl chloride (PVC) or vinyl materials, which may contain up to $40 \%$ DEHP. DEHP is present in plastic products such as tablecloths, shower curtains, garden hoses, rainwear, dolls, shoes, packaging film and sheets, medical tubing, and blood storage bags ${ }^{20,136}$. DEHP has been reported as a principal plasticizer residue contaminant of blood storage ${ }^{145}$ since it leaks from the PVC tube into the blood. DEHP also accumulates in the environment e.g. within the groundwater and may therefore end up in the food chain as well. DEHP has been classified by IARC, NTP and EPA $20,121,122$ as a possible human carcinogen. It has been observed to be metabolized within the liver to mono(2-ethylhexyl) phthalate (MEHP) ${ }^{145}$. The potential mode of action of DEHP exposure in rodents has been attributed to its role as a peroxisome proliferator, thereby activating the nuclear receptor peroxisome proliferator-activated receptor alpha (PPAR- $\alpha$ ), leading to increased cell proliferation, decreased apoptosis, oxidative DNA damage, and selective clonal expansion of the initiated cells ${ }^{146}$. For humans this mechanism is less clear.

\section{Cyclosporine A}

Cyclosporine A (CsA), a cyclic polypeptide of 11 amino acids, is produced by the fungus Beauveria nivea ${ }^{147}$. CsA, an immunosuppressive agent, is frequently used to study humoral immunity and $\mathrm{T}$ cell-dependent immune mechanisms. For example those affected within transplant rejection and some forms of autoimmunity ${ }^{148}$. CsA binds with cyclophilin, a cytoplasmic receptor protein present in target cells, to form a complex, and subsequently to calcineurin to inhibit antigen-triggered signal transduction in T lymphocytes, thus reducing the expression of many lymphokines, such as interleukin-2 (IL-2), as well as expression of antiapoptotic proteins ${ }^{136,149}$. CsA has been classified by both IARC ${ }^{121}$ and NTP 20 as a human carcinogen.

\section{Phenobarbital}

Phenobarbital $(\mathrm{PhB})$ belongs to the class of organic compounds known as barbituric acid derivatives. $\mathrm{PhB}$, an agent with anticonvulsant and sedative-hypnotic properties, affects the nonselective central nervous system thereby managing all seizure disorders (e.g. epilepsy). $\mathrm{PhB}$ acts via binding to inhibitory gamma-aminobutyric acid (GABAA) subtype receptors, and modulates chloride currents through receptor channels. It also inhibits glutamate induced depolarizations 150,151 . Two out of three in vitro genotoxicity assays gave an equivocal results (positive or negative, weak response) and the in vitro mouse lymphoma assay (MLA) gave a positive result. In vivo genotoxicity assays were however negative. Although limited evidence exists for humans, $\mathrm{PhB}$ is classified as a non-genotoxic and possible human carcinogen by IARC ${ }^{121}$.

\section{Wy 14,643}

Wy-14,643 (Wy), a synthetic thiacetic acid derivative also named pirinixic acid or wyeth-14,643, was initially developed by the pharmaceutical industry to lower serum cholesterol; however it is not used within clinical applications ${ }^{20}$. Wy, a potent (rodent) hepatocarcinogen, promotes the production of peroxisomes, which are organelles that contain a variety of enzymes involved in metabolism of lipids and cholesterol. Wy mainly act via activation of the peroxisome proliferator-activated receptor alpha (PPAR- $\alpha$ ). Long-term exposure of rodents to peroxisome 
proliferators leads to increases in peroxisomes, hepatocellular proliferation, oxidative damage, suppressed apoptosis, and ultimately results in the development of hepatocellular carcinoma 5,136,152. Conclusive hepatocarcinogenesis results exist for rodents and although Wy causes toxicity in in vitro human cells the carcinogenic effect in humans is not clear. Wy is often described to act via a similar mechanism as, the before mentioned hepatocarcinogenic peroxisome proliferator, DEHP ${ }^{153,154}$. Due to its resemblance with the mode of action of DEHP (classified as possible human carcinogen) and sufficient evidence from rodent studies, this chemical is considered a non-genotoxic carcinogen ${ }^{4}$.

\section{Reserpine}

Reserpine (Res), an alkaloid produced from the roots of certain species of the Rauwolfia plant (R. serpintina or R. vomitoria), is frequently used for the treatment of hypertension. Res inhibits normal sympathetic activity in both the central and peripheral nervous system by binding to catecholamine storage vesicles. This prevents the normal storage of catecholamines and serotonin in the nerve cell, with the result being catecholamine depletion. Res is classified a human carcinogen by the NTP 20,136 .

\section{Phenacetin}

Phenacetin (Phen), an analgesic pain-relieving and fever-reducing drug, is mainly metabolized in the liver by cytochrome P450 enzymes to acetaminophen. The analgesic and antiinflammatory effect of acetaminophen is induced by inhibition of mainly cyclooxygenase 2 (COX2) ${ }^{155}$. A small amount of the acetaminophen is further metabolized via the glucuronide pathway to the extremely hepatotoxic and bioactive N-acetyl-p-benzoquinone imine (NAPQI) which is inactivated by conjugation with glutathione. High intakes of Phen or acetaminophen, may lead to accumulation of large amounts of NAPQI and depletion of glutathione leading to increased susceptibility to oxidative stress ${ }^{136,149,156,157}$. Phen is classified by IARC, NTP and EPA as a human carcinogen ${ }^{20,121,122}$.

\section{$\underline{\text { Non-genotoxic non-carcinogens }}$}

\section{Curcumin}

Curcumin (Cur), a yellow pigment from the Curcuma longa plant, has been considered a phytochemical or nutraceutical due to its strong anti-inflammatory, anti-tumor and anti-oxidant properties. Although it has not been proven to genotoxic in vivo, it gave positive results for the combination of chromosomal aberration and the micro nucleus test (CA/MN). Cur has no reported results on human carcinogenesis and is therefore regarded as non-genotoxic and noncarcinogenic in the in vivo human situation 136,158 .

\section{4-Acetylaminofluorene}

4-Acetylaminofluorene (4-AAF), is an isomer of the very potent hepatocarcinogen 2-AAF, which is biotransformed by cytochrome P450 enzymes within the liver. 4-AAF has been proven positive for the in vitro Ames mutagenicity assay and showed equivocal results for the in vitro MLA test. In vivo no genotoxicity has been shown and therefore this compound is classified as a non-genotoxic non-carcinogen ${ }^{136}$. 


\section{Quercetin}

Quercetin (Que), a polyphenolic flavonoid ubiquitously present in plant food source, has a potential chemo-preventive, anti-oxidant and anti-proliferative activity. The chemo-preventive effect of Que is potentially via the modulation of the EGFR or estrogen-receptor mediated signal transduction pathways. The main effects have been related with decreased expression of mutant p53 protein and p21-ras oncogene, induction of cell cycle arrest at the G1 phase and inhibition of heat shock protein synthesis ${ }^{159}$. Further Que has been observed epigenetic events e.g. DNA methylation ${ }^{160}$. Although Que has been found in vitro genotoxic by means of the positive Ames mutagenicity assay and a combination of $\mathrm{CA} / \mathrm{MN}$ assay, it is not genotoxic in vivo. In addition, as indicated by IARC Que is not classifiable as to its carcinogenicity to humans. Therefore Que is regarded as a non-genotoxic and non-carcinogen in humans ${ }^{121,136}$.

\section{8-bydroxyquinoline}

8-hydroxyquinoline or 8-quinolinol (8HQ), a quinoline derivative which originates in plants as well as from industrial synthesis, has been frequently used as a fungicide in agriculture and a preservative in the textile, wood, and paper industries. 8HQ possesses lipophilic and metalchelating characteristics which play a role in various biological processes, and therefore acts as an anti-neurodegenerative, anti-cancer, anti-oxidant, anti-microbial, anti-inflammatory, and anti-diabetic agent ${ }^{161}$. Even though $8 \mathrm{HQ}$ has been proven in vitro genotoxic based on positive results within the Ames mutagenicity assay, MLA and the combined CA/MN tests, it has no genotoxic properties in vivo. Furthermore, according to the IARC 8HQ is not classifiable as to its carcinogenicity to humans ${ }^{121,136}$.

\section{Resorcinol}

Resorcinol (RR) or 1,3-benzenediol, a natural occurring phenol in argan oil and a synthetically produced agent, has anti-septic and disinfectant properties and is therefore used within all kinds of ointments as a treatment against chronic skin diseases such as acne, psoriasis and eczema. Also RR may be included in hair dyes and sun tan lotions ${ }^{136}$. RR may be converted in the liver by cytochrome P450 enzymes to a glucuronide conjugate form ${ }^{162}$. Even though RR has been proven positive for genotoxicity in vitro within the MLA and the combination of $\mathrm{CA} / \mathrm{MN}$ test, it does not seem to possess any genotoxic characteristics in vivo ${ }^{136}$. According to IARC, RR is not classifiable as to its carcinogenicity to humans ${ }^{121}$.

\section{Ampicillin tribydrate}

Ampicillin trihydrate (AmpC), a prototypic drug which is part of the aminopenicillins family, is mainly applied as an anti-bacterial agent against both gram-positive and gram-negative bacteria. AmpC is non-genotoxic and non-carcinogenic ${ }^{136,149}$.

\section{Diclofenac}

Diclofenac (Diclo), a highly potent member of the class of nonsteroidal anti-inflammatory drugs (NSAIDs), is frequently used as a COX1 and COX 2 inhibiting anti-inflammatory and analgesic pain-reducing agent. Diclo is metabolized in the liver by the cytochrome P450 enzymes into its primary metabolite; 4-hydroxydiclofenac. The drug has not been classified as a potential human carcinogen and is therefore regarded as a non-genotoxic and non-carcinogen 136,149 . 


\section{Aim, objectives and outline of the thesis}

The role of environmental factors, such as chemical carcinogens, in the development of HCC should be investigated in more detail to better understand the underlying mechanisms leading to this disease and to predict hepatic cancer risks in relation to (foreseen) exposure to such carcinogens. In the past few years, microRNAs have started a revolution in molecular biology and emerged as key players in (chemical) carcinogenesis. For these reasons, it is extremely important to understand the physiological and disease-associated mechanisms underlying the regulation of these small, single-stranded RNAs. Some microRNAs are downregulated in cancer and act as tumor suppressor genes which led to the hypothesis that microRNAs could be involved in complicated regulatory networks. Furthermore, in cancers such as HCC, aberrant DNA hypermethylation of tumor suppressor genes, global genomic DNA hypomethylation, and disruption of the histone modification patterns such as acetylation are the main epigenetic alterations, and have consequently been widely studied. Evidently, complex interplay between epigenetic factors and microRNAs has been suggested, and this may be deregulated upon exposure to carcinogenic chemicals.

The aim of this research project therefore is to elucidate the participation of microRNA and epigenetic changes in transcriptomic responses induced by genotoxic and non-genotoxic compounds in in vitro liver models. The liver models comprise a human cell line (HepG2 cells) and human and mouse primary hepatocytes (PHH and PMH). The unraveling of the mRNAmicroRNA complexes and epigenetic events involved in carcinogen response at the molecular and biological pathway and network level is expected to improve the mechanistic understanding of chemical carcinogenicity, in particular with regard to the onset of HCC. Furthermore, results from this thesis may advance chemical risk assessment and chemical safety predictions concerning genotoxic and (non-genotoxic) carcinogenic exposures in humans.

Consequently, the objectives of the present thesis are:

To identify (a) suitable hepatic model(s) for studying effects of (non-) genotoxic compounds and to explore the robust toxic response across these models.

To determine in these liver models discriminative and compound-specific responses following genotoxic or non-genotoxic carcinogen exposure.

To gain more insight into the role of microRNAs upon exposure to different classes of carcinogens and to test the usefulness of microRNA-based prediction analysis for genotoxicity and (non-genotoxic) carcinogenicity in vivo, by investigating a large set of compounds.

To combine knowledge on post-transcriptional and transcriptional networks involved in genotoxicity, evaluated on the microRNA and mRNA level, with information on epigenetic changes (DNA methylation).

To evaluate the persistent effects of genotoxic carcinogen treatment on the microRNA and mRNA expression level and the DNA methylation status.

In order to meet these objectives, the outline of this thesis is as follows:

In order to gain insight into the robustness of the genotoxic response and the mechanisms involved, three frequently used in vitro models (HepG2, PMH and $\mathrm{mESC}$ ) treated with the model compound cisplatin, were compared based on their transcriptomics responses obtained after several exposure periods as described in Chapter 2. This chapter highlights the conserved patterns in response to a model genotoxic agent on different regulatory levels, 
across all models but also describes the differences in response patterns between the respective in vitro cell models.

Chapter 3 focusses on evaluating microRNA and mRNA expression profiles following exposure to three genotoxic and three non-genotoxic carcinogens in the mouse in vitro model PMH. Both discriminative responses between the two groups of compounds as well as compound-specific responses are studied.

In Chapter 4, the set of microRNA and mRNA data, obtained following exposure to any of the six compounds, has been extended by another fifteen compounds. Each of these additional compounds belongs to either of the two classes investigated in Chapter 3. Since PMH have previously been proven successful for predicting genotoxicity in vivo, mRNA and microRNA data derived from carcinogen-exposed PMH were used to improve the current prediction outcomes for both genotoxicity as well as (non-genotoxic) carcinogenicity in vivo.

In order to expand our knowledge on the complex post-transcriptional and transcriptional networks involved in genotoxicity, in Chapter $\mathbf{5}$ also epigenetic changes in combination with microRNA and mRNA levels were investigated by analyzing a pool of primary human hepatocytes $(\mathrm{PHH})$ following a low and moderate toxic challenge of 5 days with aflatoxin $\mathrm{B} 1$ (AFB1).

Chapter 6 describes, in addition to the effects in $\mathrm{PHH}$ after a 5-day exposure period to a moderate dose of AFB1 exposure, the persistent changes in the transcriptome and the microRNAome after a 3-day washout period. This chapter describes microRNA-mRNA networks which are relevant for various HCC-related events.

In Chapter 7, the results obtained after a 5-day challenge with a low dose of AFB1, are extended with transcriptomic results and methylation changes measured after a 3-day washout period during which the carcinogen exposure was withdrawn. This chapter describes persistently expressed genes with a change in their methylation status which are relevant for the early onset of HCC.

This thesis ends with Chapter $\mathbf{8}$ in which the work presented in the previous chapters is summarized and the results of this work and the future perspectives are discussed. 


\section{References}

1.

Stewart, B.W., Wild, C., International Agency for Research on Cancer \& World Health Organization. World cancer report 2014, xiv, 630 pages (International Agency for Research on Cancer; WHO Press, Lyon, France; Geneva, Switzerland, 2014).

Ferlay, J. et al. Cancer incidence and mortality worldwide: sources, methods and major patterns in GLOBOCAN 2012. Int J Cancer 136, E359-86 (2015).

World Health Organization. National cancer control programmes : policies and managerial guidelines, xxiii, $180 \mathrm{p}$. (World Health Organization, Geneva, 2002).

Hernandez, L.G., van Steeg, H., Luijten, M. \& van Benthem, J. Mechanisms of non-genotoxic carcinogens and importance of a weight of evidence approach. Mutat Res 682, 94-109 (2009).

Gonzalez, F.J. The peroxisome proliferator-activated receptor alpha (PPARalpha): role in hepatocarcinogenesis. Mol Cell Endocrinol 193, 71-9 (2002).

Organisation for Economic Co-operation and Development (OECD). OECD Guidelines for the Testing of Chemicals http://www.oecd.org/chemicalsafety/testing/oecdguidelinesforthetestingofchemicals.htm, (accessed on July 15, 2015) (2015).

European Chemicals Agency (ECHA). Registration, Evaluation, Authorisation and Restriction of Chemicals (REACH). http://echa.europa.eu/regulations/reach, (accessed on July 15, 2015) (2015).

European Food Safety Authority (EFSA). http://www.efsa.europa.eu/, (accessed on July 15, 2015) (2015). European Medicines Agency (EMA). http://www.ema.europa.eu/ema/, (accessed on July 15, 2015) (2015).

U.S. Food and Drug Administration (FDA). http://www.fda.gov/, (accessed on July 15, 2015) (2015). Walmsley, R.M. \& Billinton, N. How accurate is in vitro prediction of carcinogenicity? British Journal of Pharmacology 162, 1250-1258 (2011).

Ames, B.N., Lee, F.D. \& Durston, W.E. An improved bacterial test system for the detection and classification of mutagens and carcinogens. Proc Natl Acad Sci US A 70, 782-6 (1973).

Hozier, J., Sawyer, J., Moore, M., Howard, B. \& Clive, D. Cytogenetic analysis of the L5178Y/TK+/- leads to TK-/- mouse lymphoma mutagenesis assay system. Mutat Res 84, 169-81 (1981).

Muller-Tegethoff, K., Kasper, P. \& Muller, L. Evaluation studies on the in vitro rat hepatocyte micronucleus assay. Mutat Res 335, 293-307 (1995).

Shelby, M.D. \& Witt, K.L. Comparison of results from mouse bone marrow chromosome aberration and micronucleus tests. Environ Mol Mutagen 25, 302-13 (1995).

Kirkland, D. \& Speit, G. Evaluation of the ability of a battery of three in vitro genotoxicity tests to discriminate rodent carcinogens and non-carcinogens III. Appropriate follow-up testing in vivo. Mutat Res 654, 114-32 (2008).

Kirkland, D. et al. How to reduce false positive results when undertaking in vitro genotoxicity testing and thus avoid unnecessary follow-up animal tests: Report of an ECVAM Workshop. Mutat Res 628, 31-55 (2007).

Kirkland, D., Kasper, P., Muller, L., Corvi, R. \& Speit, G. Recommended lists of genotoxic and nongenotoxic chemicals for assessment of the performance of new or improved genotoxicity tests: a follow-up to an ECVAM workshop. Mutat Res 653, 99-108 (2008).

. European Union Reference Laboratory for Alternatives to Animal Testing (EURL-ECVAM). ecvam.jrc.ec.europa.eu/, (accessed on July 15, 2015) (2015).

US Department of Health and Human Services. Public Health Service. National Toxicology Program (NTP). Report on Carcinogens. http://ntp.niehs.nih.gov/pubhealth/roc/roc13/index.html, (accessed on November 14, 2014) (2014).

Ennever, F.K. \& Lave, L.B. Implications of the lack of accuracy of the lifetime rodent bioassay for predicting human carcinogenicity. Regul Toxicol Pharmacol 38, 52-7 (2003).

Russell, W.M.S. \& Burch, R.L. The principles of humane experimental technique, 238 p. (Methuen, London,, 1959). Piersma, A.H. et al. A critical appraisal of the process of regulatory implementation of novel in vivo and in vitro methods for chemical hazard and risk assessment. Crit Rev Toxicol 44, 876-94 (2014).

Commandeur, J.N., Stijntjes, G.J. \& Vermeulen, N.P. Enzymes and transport systems involved in the formation and disposition of glutathione S-conjugates. Role in bioactivation and detoxication mechanisms of xenobiotics. Pharmacol Rev 47, 271-330 (1995).

\section{Parkinson, A. Biotransformation of xenobiotics, (McGraw-Hill New York, 2001).}

Castell, J.V., Jover, R., Martinez-Jimenez, C.P. \& Gomez-Lechon, M.J. Hepatocyte cell lines: their use, scope and limitations in drug metabolism studies. Expert Opin Drug Metab Toxicol 2, 183-212 (2006). Gomez-Lechon, M.J., Donato, M.T., Castell, J.V. \& Jover, R. Human hepatocytes in primary culture: the choice to investigate drug metabolism in man. Curr Drug Metab 5, 443-62 (2004). 
28.

29.

30.

31.

32.

33.

34.

35.

36.

37.

38.

39.

40.

41.

43.

44.

45.

46.

47.

48.

49.

50.

51.

53.

54.

55.

Hewitt, N.J. et al. Primary hepatocytes: current understanding of the regulation of metabolic enzymes and transporter proteins, and pharmaceutical practice for the use of hepatocytes in metabolism, enzyme induction, transporter, clearance, and hepatotoxicity studies. Drug Metab Rev 39, 159-234 (2007).

Jetten, M.J., Kleinjans, J.C., Claessen, S.M., Chesne, C. \& van Delft, J.H. Baseline and genotoxic compound induced gene expression profiles in HepG2 and HepaRG compared to primary human hepatocytes. Toxicol In Vitro 27, 2031-40 (2013).

Mathijs, K. et al. Assessing the metabolic competence of sandwich-cultured mouse primary hepatocytes. Drug Metab Dispos 37, 1305-11 (2009).

Mathijs, K. et al. Discrimination for genotoxic and nongenotoxic carcinogens by gene expression profiling in primary mouse hepatocytes improves with exposure time. Toxicol Sci 112, 374-84 (2009).

Jennen, D.G. et al. Comparison of HepG2 and HepaRG by whole-genome gene expression analysis for the purpose of chemical hazard identification. Toxicol Sci 115, 66-79 (2010).

Westerink, W.M. \& Schoonen, W.G. Phase II enzyme levels in HepG2 cells and cryopreserved primary human hepatocytes and their induction in HepG2 cells. Toxicol In Vitro 21, 1592-602 (2007).

Magkoufopoulou, C. et al. A transcriptomics-based in vitro assay for predicting chemical genotoxicity in vivo. Carcinogenesis (2012).

. Jennen, D. et al. Integrating transcriptomics and metabonomics to unravel modes-of-action of 2,3,7,8tetrachlorodibenzo-p-dioxin (TCDD) in HepG2 cells. BMC Syst Biol 5, 139 (2011).

Burczynski, M.E. et al. Toxicogenomics-based discrimination of toxic mechanism in HepG2 human hepatoma cells. Toxicol Sci 58, 399-415 (2000).

Hockley, S.L. et al. Interlaboratory and interplatform comparison of microarray gene expression analysis of HepG2 cells exposed to benzo(a)pyrene. OMICS 13, 115-25 (2009).

Lizarraga, D. et al. Benzo[a]pyrene-induced changes in microRNA-mRNA networks. Chem Res Toxicol25, 838-49 (2012).

Staal, Y.C., van Herwijnen, M.H., van Schooten, F.J. \& van Delft, J.H. Modulation of gene expression and DNA adduct formation in HepG2 cells by polycyclic aromatic hydrocarbons with different carcinogenic potencies. Carcinogenesis 27, 646-55 (2006).

van Delft, J.H. et al. Discrimination of genotoxic from non-genotoxic carcinogens by gene expression profiling. Carcinogenesis 25, 1265-76 (2004).

Van Summeren, A., Renes, J., Mariman, E.C.M., Kleinjans, J.C.S. \& van Delft, J.H.M. Response to Pathophysiological Relevance of Proteomics Investigations of Drug-Induced Hepatotoxicity in HepG2 Cells. Toxicological Sciences 121, 431-433 (2011).

Stummann, T.C. \& Bremer, S. Embryonic stem cells in safety pharmacology and toxicology. Adv Exp Med Biol 745, 14-25 (2012).

Davila, J.C. et al. Use and application of stem cells in toxicology. Toxicol Sci 79, 214-23 (2004).

Hendriks, G. et al. The ToxTracker assay: novel GFP reporter systems that provide mechanistic insight into the genotoxic properties of chemicals. Toxicol Sci (2011).

Wobus, A.M. \& Loser, P. Present state and future perspectives of using pluripotent stem cells in toxicology research. Arch Toxicol 85, 79-117 (2011).

Yildirimman, R. et al. Human embryonic stem cell derived hepatocyte-like cells as a tool for in vitro hazard assessment of chemical carcinogenicity. Toxicol Sci 124, 278-90 (2011).

Pines, A. et al. Global phosphoproteome profiling reveals unanticipated networks responsive to cisplatin treatment of embryonic stem cells. Mol Cell Biol 31, 4964-77 (2011).

Bremer, S., Pellizzer, C., Coecke, S., Paparella, M. \& Catalani, P. Detection of the embryotoxic potential of cyclophosphamide by using a combined system of metabolic competent cells and embryonic stem cells. Altern Lab Anim 30, 77-85 (2002).

- National Research Council (U.S.). Committee on Applications of Toxicogenomic Technologies to Predictive Toxicology. Applications of toxicogenomic technologies to predictive toxicology and risk assessment, xxii, 275 p. (National Academies Press, Washington, D.C., 2007).

Schena, M., Shalon, D., Davis, R.W. \& Brown, P.O. Quantitative monitoring of gene expression patterns with a complementary DNA microarray. Science 270, 467-70 (1995).

Aubrecht, J. \& Caba, E. Gene expression profile analysis: an emerging approach to investigate mechanisms of genotoxicity. Pharmacogenomics 6, 419-28 (2005).

Ellinger-Ziegelbauer, H., Aubrecht, J., Kleinjans, J.C. \& Ahr, H.J. Application of toxicogenomics to study mechanisms of genotoxicity and carcinogenicity. Toxicol Lett 186, 36-44 (2009).

Heijne, W.H., Kienhuis, A.S., van Ommen, B., Stierum, R.H. \& Groten, J.P. Systems toxicology: applications of toxicogenomics, transcriptomics, proteomics and metabolomics in toxicology. Expert Rev Proteomics 2, 767-80 (2005).

Hochstenbach, K. et al. Transcriptomic fingerprints in human peripheral blood mononuclear cells indicative of genotoxic and non-genotoxic carcinogenic exposure. Mutat Res 746, 124-34 (2012).

vivo. Carcinogenesis 33, 1421-9 (2012). 
56. Nie, A.Y. et al. Predictive toxicogenomics approaches reveal underlying molecular mechanisms of nongenotoxic carcinogenicity. Mol Carcinog 45, 914-33 (2006).

57. Fielden, M.R., Brennan, R. \& Gollub, J. A gene expression biomarker provides early prediction and mechanistic assessment of hepatic tumor induction by nongenotoxic chemicals. Toxicol Sci 99, 90-100 (2007).

58. Holsapple, M.P. et al. Mode of action in relevance of rodent liver tumors to human cancer risk. Toxicol $S_{c i}$ 89, 51-6 (2006).

59. Goodman, J.I. et al. What do we need to know prior to thinking about incorporating an epigenetic evaluation into safety assessments? Toxicol Sci 116, 375-81 (2010).

60. Mirbahai, L. \& Chipman, J.K. Epigenetic memory of environmental organisms: a reflection of lifetime stressor exposures. Mutat Res Genet Toxicol Environ Mutagen 764-765, 10-7 (2014).

61. Bartel, D. MicroRNAs: genomics, biogenesis, mechanism, and function. Cell 116, 281 - 297 (2004).

62. Roux, J., Gonzalez-Porta, M. \& Robinson-Rechavi, M. Comparative analysis of human and mouse expression data illuminates tissue-specific evolutionary patterns of miRNAs. Nucleic Acids Res 40, 5890-900 (2012).

63. Fazi, F. \& Nervi, C. MicroRNA: basic mechanisms and transcriptional regulatory networks for cell fate determination. Cardiovasc Res 79, 553-61 (2008).

64. Winter, J., Jung, S., Keller, S., Gregory, R.I. \& Diederichs, S. Many roads to maturity: microRNA biogenesis pathways and their regulation. Nat Cell Biol 11, 228-34 (2009).

65. Ha, M. \& Kim, V.N. Regulation of microRNA biogenesis. Nat Rev Mol Cell Biol 15, 509-24 (2014).

66. Jakymiw, A. et al. The role of GW/P-bodies in RNA processing and silencing. J Cell Sci 120, 1317-23 (2007).

67. Wienholds, E. \& Plasterk, R.H.A. MicroRNA function in animal development. FEBS Letters 579, 59115922 (2005).

68. Sun, J., Lu, H., Wang, X. \& Jin, H. MicroRNAs in hepatocellular carcinoma: regulation, function, and clinical implications. ScientificW orldJournal 2013, 924206 (2013).

69. Gramantieri, L. et al. MicroRNA involvement in hepatocellular carcinoma. J Cell Mol Med 12, 2189-204 (2008).

70. Chen, R.X., Xia, Y.H., Xue, T.C. \& Ye, S.L. Suppression of microRNA-96 expression inhibits the invasion of hepatocellular carcinoma cells. Mol Med Rep 5, 800-4 (2012).

71. Fornari, F. et al. MiR-221 controls CDKN1C/p57 and CDKN1B/p27 expression in human hepatocellular carcinoma. Oncogene 27, 5651-61 (2008).

72. Giordano, S. \& Columbano, A. MicroRNAs: new tools for diagnosis, prognosis, and therapy in hepatocellular carcinoma? Hepatology 57, 840-7 (2013).

73. Hou, J. et al. Identification of miRNomes in human liver and hepatocellular carcinoma reveals miR-199a/b$3 p$ as therapeutic target for hepatocellular carcinoma. Cancer Cell 19, 232-43 (2011).

74. Huang, X.Y. et al. MicroRNA-429 Modulates Hepatocellular Carcinoma Prognosis and Tumorigenesis. Gastroenterol Res Pract 2013, 804128 (2013).

75. Pineau, P. et al. miR-221 overexpression contributes to liver tumorigenesis. Proc Natl Acad Sci U S A 107, 264-9 (2010).

76. Yamamoto, Y. et al. MicroRNA-500 as a potential diagnostic marker for hepatocellular carcinoma. Biomarkers 14, 529-38 (2009).

77. Bai, S. et al. MicroRNA-122 Inhibits Tumorigenic Properties of Hepatocellular Carcinoma Cells and Sensitizes These Cells to Sorafenib. The Journal of Biological Chemistry 284, 32015-32027 (2009).

78.

79. Tomimaru, Y. et al. MicroRNA-21 induces resistance to the anti-tumour effect of interferon- $\alpha / 5$ fluorouracil in hepatocellular carcinoma cells. British Journal of Cancer 103, 1617-1626 (2010).

80. Izzotti, A. \& Pulliero, A. The effects of environmental chemical carcinogens on the microRNA machinery. International Journal of Hygiene and Environmental Health 217, 601-627 (2014).

81. Izzotti, A. et al. Dose-responsiveness and persistence of microRNA expression alterations induced by cigarette smoke in mouse lung. Mutat Res 717, 9-16 (2011).

82. Izzotti, A. et al. Downregulation of microRNA expression in the lungs of rats exposed to cigarette smoke. FASEB J 23, 806-12 (2009).

83. Li, E. Chromatin modification and epigenetic reprogramming in mammalian development. Nat Rev Genet 3 , 662-73 (2002).

84. Fisher, A.G. Cellular identity and lineage choice. Nat Rev Immunol 2, 977-82 (2002).

85. Bird, A. DNA methylation patterns and epigenetic memory. Genes Dev 16, 6-21 (2002).

86. Ehrlich, M. Expression of various genes is controlled by DNA methylation during mammalian development. J Cell Biochem 88, 899-910 (2003).

87. Legler, J. Epigenetics: an emerging field in environmental toxicology. Integr Environ Assess Manag 6, 314-5 (2010). 
88.

89.

90.

91.

92.

93.

94.

95.

96.

97.

98.

99.

100.

101.

102.

103.

104.

105.

106.

107.

108.

109

110.

111.

114.

Rajender, S., Avery, K. \& Agarwal, A. Epigenetics, spermatogenesis and male infertility. Mutat Res 727, 6271 (2011).
Holliday, R. \& Grigg, G.W. DNA methylation and mutation. Mutat Res 285, $61-7$ (1993).

Jin, B., Li, Y. \& Robertson, K.D. DNA Methylation: Superior or Subordinate in the Epigenetic Hierarchy? Genes \& Cancer 2, 607-617 (2011).

Suzuki, M.M. \& Bird, A. DNA methylation landscapes: provocative insights from epigenomics. Nat Rev Genet 9, 465-76 (2008).

Bird, A.P. CpG-rich islands and the function of DNA methylation. Nature 321, 209-13 (1986).

Jeltsch, A. Beyond Watson and Crick: DNA methylation and molecular enzymology of DNA methyltransferases. Chembiochem 3, 274-93 (2002).

Zeisel, S.H. Epigenetic mechanisms for nutrition determinants of later health outcomes. Am J Clin Nutr 89, 1488S-1493S (2009).

McKay, J.A., Williams, E.A. \& Mathers, J.C. Folate and DNA methylation during in utero development and aging. Biochem Soc Trans 32, 1006-7 (2004).

Brown, R. \& Strathdee, G. Epigenomics and epigenetic therapy of cancer. Trends in Molecular Medicine 8 , S43-S48 (2002).

Singh, S.M., Murphy, B. \& O'Reilly, R.L. Involvement of gene-diet/drug interaction in DNA methylation and its contribution to complex diseases: from cancer to schizophrenia. Clin Genet 64, 451-60 (2003).

Santini, V., Kantarjian, H.M. \& Issa, J.P. Changes in DNA methylation in neoplasia: pathophysiology and therapeutic implications. Ann Intern Med 134, 573-86 (2001).

Reik, W., Dean, W. \& Walter, J. Epigenetic reprogramming in mammalian development. Science 293, 108993 (2001).

Zhu, H. et al. Lsh is involved in de novo methylation of DNA. EMBOJ 25, 335-45 (2006).

Crider, K.S., Yang, T.P., Berry, R.J. \& Bailey, L.B. Folate and DNA methylation: a review of molecular mechanisms and the evidence for folate's role. Adv Nutr 3, 21-38 (2012).

Baylin, S.B., Herman, J.G., Graff, J.R., Vertino, P.M. \& Issa, J.P. Alterations in DNA methylation: a fundamental aspect of neoplasia. Adv Cancer Res 72, 141-96 (1998).

Kim, Y.I. Nutritional epigenetics: impact of folate deficiency on DNA methylation and colon cancer susceptibility. J Nutr 135, 2703-9 (2005).

Sceusi, E.L., Loose, D.S. \& Wray, C.J. Clinical implications of DNA methylation in hepatocellular carcinoma. HPB : The Official Journal of the International Hepato Pancreato Biliary Association 13, 369-376 (2011). Knudson, A.G. Hereditary cancer: two hits revisited. J Cancer Res Clin Oncol 122, 135-40 (1996).

Feinberg, A.P., Ohlsson, R. \& Henikoff, S. The epigenetic progenitor origin of human cancer. Nat Rev Genet 7, 21-33 (2006).

Kopp-Schneider, A., Portier, C. \& Bannasch, P. A model for hepatocarcinogenesis treating phenotypical changes in focal hepatocellular lesions as epigenetic events. Math Biosci 148, 181-204 (1998).

Ushijima, T. Epigenetic field for cancerization. J Biochem Mol Biol 40, 142-50 (2007).

Kondo, Y. et al. Genetic instability and aberrant DNA methylation in chronic hepatitis and cirrhosis--A comprehensive study of loss of heterozygosity and microsatellite instability at 39 loci and DNA hypermethylation on $8 \mathrm{CpG}$ islands in microdissected specimens from patients with hepatocellular carcinoma. Hepatology 32, 970-9 (2000).

0. Farazi, P.A. \& DePinho, R.A. Hepatocellular carcinoma pathogenesis: from genes to environment. Nat Rev Cancer 6, 674-87 (2006).

1. Zhang, Y.J. et al. High frequency of promoter hypermethylation of RASSF1A and p16 and its relationship to aflatoxin B1-DNA adduct levels in human hepatocellular carcinoma. Mol Carcinog 35, 85-92 (2002). Maeta, Y., Shiota, G., Okano, J. \& Murawaki, Y. Effect of promoter methylation of the p16 gene on phosphorylation of retinoblastoma gene product and growth of hepatocellular carcinoma cells. Tumour Biol 26, 300-5 (2005).

Lee, S. et al. Aberrant CpG island hypermethylation along multistep hepatocarcinogenesis. Am J Pathol 163, 1371-8 (2003).

4. Hernandez-Vargas, H. et al. Hepatocellular Carcinoma Displays Distinct DNA Methylation Signatures with Potential as Clinical Predictors. PLoS ONE 5, e9749 (2010).

Cheng, Y. et al. Correlation of CpG island methylator phenotype with poor prognosis in hepatocellular carcinoma. Exp Mol Pathol 88, 112-7 (2010).

6. Pavanello, S. et al. Global and gene-specific promoter methylation changes are related to anti-B[a]PDEDNA adduct levels and influence micronuclei levels in polycyclic aromatic hydrocarbon-exposed individuals. Int J Cancer 125, 1692-7 (2009).

7. Hou, L., Zhang, X., Wang, D. \& Baccarelli, A. Environmental chemical exposures and human epigenetics. Int J Epidemiol 41, 79-105 (2012).

van Breda, S.G. et al. Epigenetic mechanisms underlying arsenic-associated lung carcinogenesis. Arch Toxicol (2014). 
119. Lee, Y.W. et al. Carcinogenic nickel silences gene expression by chromatin condensation and DNA methylation: a new model for epigenetic carcinogens. Mol Cell Biol 15, 2547-57 (1995).

120. Heijmans, B.T. et al. Persistent epigenetic differences associated with prenatal exposure to famine in humans. Proc Natl Acad Sci US A 105, 17046-9 (2008).

121. International Agency for Research on Cancer (IARC). IARC Monographs on the Evaluation of Carcinogenic Risks to Humans.

http://monographs.iarc.fr/ENG/Classification/ClassificationsGroupOrder.pdf, (accessed on November 14, 2014) (2014). Environmental Protection Agency, U.S. Integrated Risk Information System (IRIS) http://www.epa.gov/IRIS/(2015).

123. Liu, Y. \& Wu, F. Global burden of aflatoxin-induced hepatocellular carcinoma: a risk assessment. Environ Health Perspect 118, 818-24 (2010). Kew, M.C. Aflatoxins as a cause of hepatocellular carcinoma. J Gastrointestin Liver Dis 22, 305-10 (2013). Wu, H.C. \& Santella, R. The Role of Aflatoxins in Hepatocellular Carcinoma. Hepat Mon 12, e7238 (2012). Bedard, L.L. \& Massey, T.E. Aflatoxin B1-induced DNA damage and its repair. Cancer Lett 241, 174-83 (2006).

127. Guengerich, F.P. et al. Activation and detoxication of aflatoxin B1. Mutation Research/Fundamental and Molecular Mechanisms of Mutagenesis 402, 121-128 (1998). Gursoy-Yuzugullu, O., Yuzugullu, H., Yilmaz, M. \& Ozturk, M. Aflatoxin genotoxicity is associated with a defective DNA damage response bypassing p53 activation. Liver Int 31, 561-71 (2011).

129. Hall, M.D., Okabe, M., Shen, D.W., Liang, X.J. \& Gottesman, M.M. The role of cellular accumulation in determining sensitivity to platinum-based chemotherapy. Annu Rev Pharmacol Toxicol 48, 495-535 (2008). Galluzzi, L. et al. Molecular mechanisms of cisplatin resistance. Oncogene (2011).

131. Zicca, A. et al. Reduction of cisplatin hepatotoxicity by procainamide hydrochloride in rats. European journal of pharmacology 442, 265-72 (2002).

132. Miyata, M., Furukawa, M., Takahashi, K., Gonzalez, F.J. \& Yamazoe, Y. Mechanism of 7,12dimethylbenz[a]anthracene-induced immunotoxicity: role of metabolic activation at the target organ. Jpn J Pharmacol 86, 302-9 (2001).

133. Weimer, T.L. et al. Influence of beta-naphthoflavone on 7,12-dimethylbenz(a)anthracene metabolism, DNA adduction, and tumorigenicity in rainbow trout. Toxicol Sci 57, 217-28 (2000).

134. DiGiovanni, J., Sina, J.F., Ashurst, S.W., Singer, J.M. \& Diamond, L. Benzo(a)pyrene and 7,12dimethylbenz(a)anthracene metabolism and DNA adduct formation in primary cultures of hamster epidermal cells. Cancer Res 43, 163-70 (1983).

135. Singhal, R., Badger, T.M. \& Ronis, M.J. Reduction in 7,12-dimethylbenz[a]anthracene-induced hepatic cytochrome-P450 $1 \mathrm{~A} 1$ expression following soy consumption in female rats is mediated by degradation of the aryl hydrocarbon receptor. J Nutr 137, 19-24 (2007).

136. U.S. National Library of Medicine. Toxnet Toxicological Data Network (CCRIS,HSDB, ChemIDplus and Genetox). http://toxnet.nlm.nih.gov/, (accessed on November 14, 2014) (2014).

137. p-Cresidine. Rep Carcinog 12, 122-3 (2011).

138. Katika, M.R., Hendriksen, P.J., de Ruijter, N.C., van Loveren, H. \& Peijnenburg, A. Immunocytological and biochemical analysis of the mode of action of bis (tri-n-butyltin) tri-oxide (TBTO) in Jurkat cells. Toxicol Lett 212, 126-36 (2012).

139. van Kol, S.W., Hendriksen, P.J., van Loveren, H. \& Peijnenburg, A. Transcriptomics analysis of primary mouse thymocytes exposed to bis(tri-n-butyltin)dioxide (TBTO). Toxicology 296, 37-47 (2012).

140. Davis, A. et al. Evaluation of the genetic and embryotoxic effects of bis(tri-n-butyltin)oxide (TBTO), a broad-spectrum pesticide, in multiple in vivo and in vitro short-term tests. Mutat Res 188, 65-95 (1987).

141. Schecter, A., Birnbaum, L., Ryan, J.J. \& Constable, J.D. Dioxins: an overview. Environ Res 101, 419-28 (2006).

142. Nichols, K.C., Schenkel, L. \& Benson, H. 17 beta-estradiol for postmenopausal estrogen replacement therapy. Obstet Gynecol Surv 39, 230-45 (1984).

143. Safe, S. Transcriptional activation of genes by 17 beta-estradiol through estrogen receptor-Sp1 interactions. Vitam Horm 62, 231-52 (2001).

144. Thomas, C.G., Strom, A., Lindberg, K. \& Gustafsson, J.A. Estrogen receptor beta decreases survival of p53-defective cancer cells after DNA damage by impairing G(2)/M checkpoint signaling. Breast Cancer Res Treat 127, 417-27 (2011).

145. Peck, C.C. \& Albro, P.W. Toxic potential of the plasticizer Di(2-ethylhexyl) phthalate in the context of its disposition and metabolism in primates and man. Environ Health Perspect 45, 11-7 (1982).

146. Rusyn, I., Peters, J.M. \& Cunningham, M.L. Modes of action and species-specific effects of di-(2ethylhexyl)phthalate in the liver. Crit Rev Toxicol36, 459-79 (2006).

147. Thomson, A.W., Smith, S.W. \& Chappell, L.H. Cyclosporin A: immune suppressant and antiparasitic agent. Parasitol Today 2, 288-90 (1986). 
148. Charan, S., Huegin, A.W., Cerny, A., Hengartner, H. \& Zinkernagel, R.M. Effects of cyclosporin A on humoral immune response and resistance against vesicular stomatitis virus in mice. Journal of Virology 57 , 1139-1144 (1986).

149. Goodman, L.S., Brunton, L.L., Chabner, B. \& Knollmann, B.r.C. Goodman \& Gilman's pharmacological basis of therapentics, 2084 p. (McGraw-Hill, New York, 2011).

150. Law, V. et al. DrugBank 4.0: shedding new light on drug metabolism. Nucleic Acids Res 42, D1091-7 (2014).

151. Kwan, P. \& Brodie, M.J. Phenobarbital for the treatment of epilepsy in the 21st century: a critical review. Epilepsia 45, 1141-9 (2004).

152. Woods, C.G. et al. WY-14,643-Induced Cell Proliferation and Oxidative Stress in Mouse Liver are Independent of NADPH Oxidase. Toxicological Sciences 98, 366-374 (2007).

153. Guyton, K.Z. et al. A reexamination of the PPAR-alpha activation mode of action as a basis for assessing human cancer risks of environmental contaminants. Environ Health Perspect 117, 1664-72 (2009).

154. Guyton, K.Z. et al. Improving prediction of chemical carcinogenicity by considering multiple mechanisms and applying toxicogenomic approaches. Mutat Res 681, 230-40 (2009).

155. Hinz, B., Cheremina, O. \& Brune, K. Acetaminophen (paracetamol) is a selective cyclooxygenase-2 inhibitor in man. FASEB J 22, 383-90 (2008).

156. Hinson, J.A., Roberts, D.W. \& James, L.P. Mechanisms of Acetaminophen-Induced Liver Necrosis. Handbook of experimental pharmacology, 369-405 (2010).

157. Tan, G.H., Rabbino, M.D. \& Hopper, J., Jr. IS PHENACETIN A NEPHROTOXIN?A REPORT ON TWENTY-THREE USERS OF THE DRUG. Calif Med 101, $73-7$ (1964).

158. Hazzard, W.R. \& Halter, J.B. Hazzard's geriatric medicine and gerontology, xxviii, 1,634 p., 12 p. of plates (McGraw-Hill Medical, New York, 2009).

159. Sharmila, G. et al. Chemopreventive effect of quercetin, a natural dietary flavonoid on prostate cancer in in vivo model. Clin Nutr 33, 718-26 (2014).

160. Gibellini, L. et al. Quercetin and cancer chemoprevention. Evid Based Complement Alternat Med 2011, 591356 (2011).

161. Prachayasittikul, V., Prachayasittikul, S., Ruchirawat, S. \& Prachayasittikul, V. 8-Hydroxyquinolines: a review of their metal chelating properties and medicinal applications. Drug Design, Development and Therapy 7 , 1157-1178 (2013).

162. Kim, Y.C. \& Matthews, H.B. Comparative metabolism and excretion of resorcinol in male and female F344 rats. Fundam Appl Toxicol 9, 409-14 (1987). 


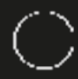

O

0

0
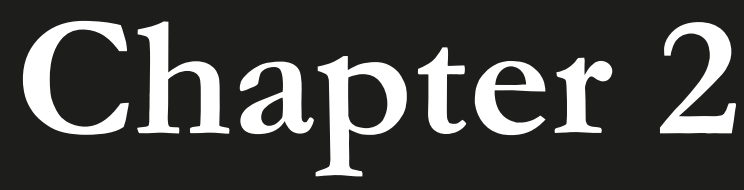

00



()

Characterization of cisplatin-induced transcriptomics responses in primary

() mouse hepatocytes, HepG 2 cells and mouse embryonic stem cells shows conservation of regulating transeription factor networks.

0
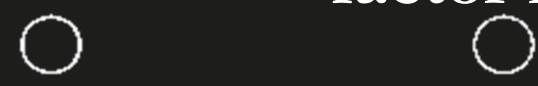

Mytagenegis. 2014 January Volume 29; Issue 1; Pages 17-26

is
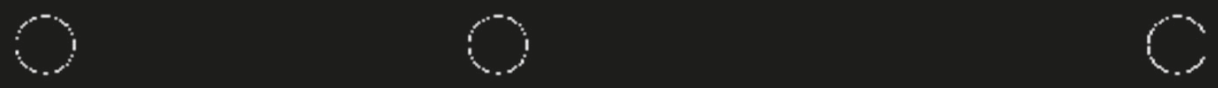

$\mathrm{O}$

00

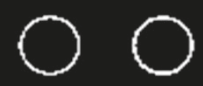

6i
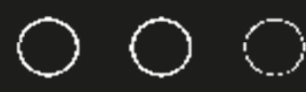

Rieswijk L.

Lizarraga D.

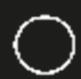

Brauers K.J.J.

Kleinjans J.C.S.

van Delft J.H.M. 


\section{$\underline{\text { Abstract }}$}

The toxic mechanisms of cisplatin have been frequently studied in many species and in vitro cell models. The Netherlands Toxicogenomics Centre focuses on developing in vitro alternatives using genomics technologies for animal-based assays on e.g. genotoxic hazards. Models such as HepG2 cells, mouse primary hepatocytes $(\mathrm{PMH})$ and mouse embryonic stem cells (mESC) are used. Our aim was to identify possibly robust conserved mechanisms between these models using cisplatin as model genotoxic agent. Transcriptomic data newly generated from HepG2 cells and PMH exposed to $7 \mu \mathrm{M}$ cisplatin for $12,24,48 \mathrm{~h}$ and $24,48 \mathrm{~h}$, respectively, were compared with published data from mESC exposed to $5 \mu \mathrm{M}$ cisplatin for $2-24 \mathrm{~h}$. Due to differences in response-time between models and marginal changes after shorter exposureperiods, we focused on 24 and 48h. At gene-level, 44 conserved differentially expressed genes (DEG) were found, involved in processes such as apoptosis, cell cycle, DNA damage response and DNA repair. Functional analysis shows that limited numbers of pathways are conserved. Transcription factor (TF) network analysis indicates 12 common TF-networks responding among all models and time-points. Four TF, HNF4- $\alpha$, SP-1, c-MYC and p53, capable of regulating $\pm 50 \%$ of all DEG, seem of equal importance in all models and exposure-periods. Here we showed that transcriptomic responses across several in vitro cell models following exposure to cisplatin is mainly determined by a conserved complex network of 4 TFs. These conserved responses are hypothesized to provide most relevant information for human toxicity prediction and may form the basis for new in vitro alternatives of risk-assessment. 


\section{Introduction}

Continuously, new chemicals and pharmaceuticals are being developed. All of these compounds first need to be tested for their toxic potential, such as possible genotoxic (GTX) properties, before they are allowed to enter the public market. Genotoxicity tests are routinely used for predicting some genotoxic properties of chemicals ${ }^{1}$. There are multiple in vitro genotoxicity assays available which are characterized by low specificity and as a result high false-positive rate ${ }^{2}$. Nowadays new tests are developed, which are aimed at being more accurate and enabling a faster toxicity testing of novel compounds. Toxicogenomics approaches, based on tools such as DNA microarrays, are used to study GTX effects of compounds by focusing on their gene expression profiles. This enables investigating mechanistic relations between these genes by studying affected molecular networks and pathways ${ }^{3}$. The Netherlands Toxicogenomics Centre focuses on developing better in vitro assays for assessing GTX hazards using genomics technologies. Several cell models are used for this, i.e. HepG2 cells, mouse primary hepatocytes (PMH) and mouse embryonic stem cells (mESC).

Many in vitro systems that are used for toxicity studies are derived from the liver, since this is an important organ for the biotransformation of many compounds into their reactive metabolites and thus it is a main target for genotoxins ${ }^{4}$. The hepatocyte systems include among others precision-cut liver slices, primary hepatocytes, hepatoma derived, immortalized or transgenic animal derived hepatic cell lines ${ }^{5}$. Primary human cells would be ideal from the perspective of human health. Donors of liver cells, however, are scarce, the degree of variability between individuals is high and the ability of differentiated cells to grow in vitro remains limited 4 . Primary hepatocytes from rodents are also frequently used, where those of mice are more metabolically stable than those of rats ${ }^{6}$. Alternatives for primary hepatocytes would be hepatic cell lines, such as the widely-used hepatoma derived HepG2 ${ }^{7-14}$, BC2 ${ }^{15}$ or the HepaRG cell line ${ }^{16}$. The advantage of hepatic cell lines is that they can continuously grow, have an almost unlimited lifespan and retain a quite stable phenotype ${ }^{5}$. Furthermore, there is less biological variation compared to primary hepatocytes. Both human HepaRG as well as HepG2 cell lines are used for genotoxicity and toxicogenomics studies ${ }^{7-14,17}$. Human HepaRG cells have the benefit that they are metabolically competent. HepG2 metabolic competence is less than that in human liver. HepG2 are known for their relatively low expression of cytochrome P450 enzymes, however they are still capable of metabolizing precarcinogenic compounds as is demonstrated for instance by their capacity to form DNA adducts ${ }^{7,9-14}$. Furthermore, their value for classifying genotoxic compounds base on global gene expression analysis, has also been shown ${ }^{8}$.

In addition, undifferentiated cell lines like mammalian embryonic stem cells (ESCs) are becoming more popular for genotoxicity studies ${ }^{18,19}$, since they are untransformed and share characteristics with cancer stem cells ${ }^{20}$. ESCs while not representing specific target organs such as the liver do not require immortalization for maintaining proliferation capacity and ESC derivatives are functional in vitro and after transplantation in vivo. Another advantage of mouse ESCs is that they can easily be genetically modified and used to generate transgenic mice ${ }^{21}$. Hence there is a wide range of in vitro systems available that all have their advantages and disadvantages in toxicology research.

Risk assessment assumes that there is a conserved mode of action and comparable toxic responses between species. Despite the fact that species differences exist in xenobiotic responses, e.g. due to metabolism differences, many biological systems act in an evolutionarily conserved way across a large number of species. With the use of more improved computational bioinformatic tools, microarray expression datasets may offer the opportunity 
to combine expression experiments from multiple species to identify genes that are not only conserved in sequence but also regulated in a similar manner across species. Orthologs are genes from different genomes that originate from a common ancestor gene by organism speciation. They are most similar by the structure of encoded proteins (compared with other genes in these genomes) and therefore should have a similar function ${ }^{22}$. Orthologs are used for comparative transcriptomic analyses within this study.

We therefore hypothesize that adverse response patterns conserved across in vitro models from different species including human and cell types, may provide the most relevant mechanistic information for toxicity in humans. The main aim of this current study is to gain insight into such conserved transcriptomic response by comparing the orthologous gene expression profiles of the three in vitro models that are used within the Netherlands Toxicogenomics Centre for developing in vitro assays for classifying GTX compounds, i.e. HepG2 cells, PMH and mESC, following exposure to a model GTX agent, namely cisplatin. The objective of our study thus is to investigate the robustness of the three in vitro cell models considered for 'omics-based classification of GTX compounds, and to study potential conserved response mechanisms between them. These results may help in identifying conserved responses at the level of biological and signal transduction pathways and of transcription factor networks, and thereby in understanding specific modes of action of genotoxins which in the long run could lead to better assays for human hazard identification.

The model GTX agent, cisplatin, which we here use, is a widely used chemotherapy drug for treating a variety of solid tumors, which does not require metabolic activation for exerting its genotoxic activity. Therefore this compound can also be used in systems of limited metabolic competence such as mouse embryonic stem cells or HepG2 cells. The cytotoxic and GTX effects of cisplatin are a consequence of the formation of DNA adducts, especially 1,2intrastrand $\mathrm{d}(\mathrm{GpG})$ cross-links . Cisplatin may enter the cell by passive diffusion and facilitated active transport. Spontaneous hydrolysis inside the cell, results in a highly reactive metabolite , which may bind to the N7 of guanosine and adenine in the DNA, causing mutations and cell death ${ }^{23}$. In many tissues and cell systems, such as HepG2 ${ }^{24}$, cisplatin leads to inhibition of replication and transcription, to the activation of the ATM/ATR- and p53-regulated DNA damage response resulting in cell growth arrest, DNA repair, and apoptosis ${ }^{25}$. Cisplatin is also known for its high occurrence of side effects, i.e. leading to nephro-, neuro- and ototoxicity and secondary cancers at multiple organs ${ }^{25}$. Hepatotoxicity has been established as one of the dose-limiting factors of cisplatin treatment ${ }^{26}$, however the mechanisms underlying this type of toxicity has not well been characterized.

In this study cisplatin-induced transcriptomic profiles of PMH, HepG2 cells and mESC were investigated. By functional analyses of modulated biochemical pathway maps, insights into the conserved mechanisms were obtained which provides information that could not be obtained when analyzing data from a single species. In particular, changes of transcription factor (TF) regulatory networks were studied, since they are thought to play a key role in evolution due to their direct impact on gene-expression levels ${ }^{17,27}$. 


\section{Material and methods}

\section{Cell culture and treatment}

\section{Human hepatocellular carcinoma cell line (HepG2)}

HepG2 cells were cultered as previously described by Staal and colleagues ${ }^{28}$. A minimallycytotoxic dose of cis-Diamineplatinum(II) dichloride (cisplatin; $\geq 99.9 \%$ purity, CAS no. 15663-27-1; Sigma-Aldrich Chemie B.V., Zwijndrecht, The Netherlands) was determined by the MTT assay ${ }^{29}$ with $72 \mathrm{~h}$ exposure, resulting in $\pm 80 \%$ viability (results are shown in Supplementary Figure 2.1A). No further doses were investigated, as based on our previous studies in HepG2 cells we are aware that time has a much larger effect on gene expression profiles than dose ${ }^{28}$. Studies of cisplatin on mESC confirmed that ${ }^{30}$. Cell cultures were harvested at $80 \%$ confluency and seeded into six-well plates $\left(1.4 \times 10^{5}\right.$ cells/well). The medium was refreshed one day before exposure. The HepG2 cells were exposed to $7 \mu \mathrm{M}$ of cisplatin (the IC20 at $72 \mathrm{~h}$ based on MTT assay) or to a vehicle control (0.5\% of PBS). This dose was selected since mostly death responses would be observed when choosing a higher dose. The cells were exposed for $12 \mathrm{~h}, 24 \mathrm{~h}$ and $48 \mathrm{~h}$ in three independent experiments. These exposure periods were selected based on the fact that HepG2 cells have a cell cycle of $\sim 20 \mathrm{~h}$ and would therefore respond more slowly than mESC, as the latter have a cell cycle of $\sim 12 \mathrm{~h}$.

\section{Primary mouse hepatocytes $(\mathrm{PMH})$}

PMH were isolated from adult male C57BL/6 mice using a perfusion method and cultured in a collagen-collagen sandwich conformation in six wells plates as previously described ${ }^{6}$. After a recovery period of 40-42h, the Dulbecco's Modified Eagle's (Gibco BRL, Breda, The Netherlands) culture medium was replaced by culture medium containing cisplatin or a vehicle control $(0.5 \%$ of PBS). A minimally-cytotoxic dose of cisplatin was established by the MTT assay ${ }^{29}$ with $48 \mathrm{~h}$ exposure, resulting in $\pm 80 \%$ viability (results are shown in Supplementary Figure 2.1A). Finally, cells were treated with $7 \mu \mathrm{M}$ cisplatin (the IC20 at $48 \mathrm{~h}$ based on MTT assay) for 24 or $48 \mathrm{~h}$ before being harvested. Independent triplicate biological experiments with hepatocytes from different mice were obtained for every time point.

\section{Mouse embryonic stem cells (mESC)}

Microarray data from a previous experiment by Kruse et al were used here ( ${ }^{30}$; ArrayExpress ETABM-89). In this experiment wild type mESC (cell line B4418 of C57BL/6 mice) were cultured on mouse embryonic fibroblast feeder layers by Kruse et al ${ }^{30}$ (Department of Toxicogenetics, LUMC, Leiden, The Netherlands). Subconfluent cultures were treated with different concentrations of cisplatin $(0,1,2,5$ and $10 \mu \mathrm{M})$ for $0,2,4,8,24 \mathrm{~h}$. As measure for cytotoxicity, apoptosis was determined by annexin $\mathrm{V} /$ propidium iodide (AV/PI) staining. Triplicate experiments were performed for all concentrations and time course experiments. The study from Kruse et al again showed that time-related effects on gene expression are more pronounced than dose-related effects. The $5 \mu \mathrm{M}$ dose at $24 \mathrm{~h}$ found in $\mathrm{mESC}$ causes a $20 \%$ increase in apoptosis (in comparison to exposure with the control). This would in theory be comparable with a $20 \%$ decrease in cell viability using MT'T. The others doses used in this study seem to cause a lower and higher increase of apoptosis $(2 \mu \mathrm{M}: 10 \%$ increase of apoptosis, $10 \mu \mathrm{M}$ : $40 \%$ increase of apoptosis) (for all apoptosis measurements see 
Supplementary Figure 2.1B). Therefore, we believe that $5 \mu \mathrm{M}$ in $\mathrm{mESC}$ is the appropriate dose for comparative transcriptomics studies.

\section{RNA isolation and quality control}

At the end of the treatment, the medium was removed and the HepG2 and PMH were harvested in Qiazol (QIAGEN Benelux B.V., Venlo, The Netherlands). Total RNA was isolated using a miRNeasy Mini Kit (QIAGEN Benelux B.V., Venlo, The Netherlands) according to the manufacturer's protocol and followed by DNase I (QIAGEN Benelux B.V., Venlo, The Netherlands) treatment. Following purification, RNA concentrations were measured by means of a NanoDrop ${ }^{\circledR}$ ND-1000 spectrophotometer (Thermo Scientific, Wilmington, USA) at 260 and $280 \mathrm{~nm}$. RNA quality and integrity were assessed by using automated gel electrophoresis on an Agilent 2100 Bioanalyzer system (Agilent Technologies Netherlands B.V., Amstelveen, The Netherlands). Only RNA samples which showed clear 18S and $28 \mathrm{~S}$ peaks and with a RNA integrity number (RIN) higher than 7.5 were used. Samples were stored at $-80^{\circ} \mathrm{C}$ until RNA hybridization.

\section{Gene expression analyses using Affymetrix arrays}

\section{Labelling and hybridization}

High-density oligonucleotide GeneChips from Affymetrix (with a large number of spotted probes) were used to measure gene expression; Human Genome U133 Plus 2.0 array for HepG2 (54675 probes), Mouse Genome 4302.0 array for PMH (45101 probes) and Mouse Genome 430A array for mESC (22621 probes). Targets for these arrays are prepared from 250 ng of total RNA by means of the GeneChip 3' IVT Express Kit according to the Affymetrix protocol (Affymetrix UK Ltd, High Wycombe, UK). Amplified, biotinylated and fragmented targets were then hybridized on to the arrays. After hybridization, arrays were washed and stained using an Affymetrix fluidics station and scanned by use of an Affymetrix GeneArray scanner.

A total of eighteen, twelve and twenty-four RNA samples, from HepG2, PMH and mESC respectively, was prepared and analyzed on GeneChip arrays (treated and control samples from each time point were in triplicate).

Normalization quality controls, including scaling factors, average intensities, present calls, background intensities, noise, and raw $Q$ values, were within acceptable limits for all chips. Hybridization controls BioB, BioC, BioD, and CreX were called present on all chips and yielded the expected increases in intensities. Data obtained in this study from HepG2 cells and PMH can be accessed through GEO Series accession number GSE38124.

\section{Data analysis}

\section{$\underline{\text { Re-annotation, normalization, and data filtering }}$}

All raw datasets from generated CEL files were first imported into R2.10.1 and run through an in-house quality control pipeline (available through ArrayAnalysis.org ${ }^{31}$ ) and then used for statistical analysis. $\mathrm{R}$ package is freely available for academic use from the Comprehensive $\mathrm{R}$ Archive Network (http://www.cran.r-project.org/). After the quality control 3 samples of the 
mESC experiments, which were a control sample of $2 \mathrm{~h}$ and $24 \mathrm{~h}$ and one replicate of $8 \mathrm{~h}$ with $5 \mu \mathrm{M}$ of cisplatin, were excluded from further analysis since they did not pass the criteria. Since two of these deviating arrays were control samples, also the matching treated samples were excluded for further analysis (11 arrays in total). For the remaining arrays probe sets were reannotated by use of custom Cell Definition Files (CDF) (version 12.1.0. http://brainarray.mbni.med.umich.edu/Brainarray/Database/CustomCDF/ 12.1.0/entrezg. asp). This resulted in a list of 16331, 17726 and 12045 unique gene IDs for PMH, HepG2 cells and mESC respectively. After re-annotation, data were normalized by Robust Multichip Average (RMA) normalization and $\log 2$ transformed using the R package "affy". Low expression genes were filtered out by using a $\log [$ intensity cut-off $]>5$. This cut-off was based on the $1^{\text {st }}$ quartile of the distribution of average intensities of all reporters. Filtering out low expressed genes reduces the amount of noise being measured and thus excludes noninformative genes ${ }^{32}{ }^{33}$. After the low expression genes were filtered out, a list of 11870, 13173 and 10705 unique gene IDs for PMH, HepG2 cells and mESC respectively were remained for further analysis.

\section{Selection of Differentially Expressed Genes (DEG)}

After filtering, the respective $\log 2$ ratios were calculated between the average intensities of the treated and control samples and significance was determined by performing a paired Student's t-test using R. Absolute fold changes of 1.5 and a $p$-value $<0.05$, unadjusted, were used for selection of DEG. No FDR was used since it has been reported within the MicroArray Quality Control (MAQC) project, that reproducibility of microarray data is higher when criteria such as fold change are used ${ }^{34}$.

\section{Gene Orthology Mapping}

The use of different species and microarray platforms may result in differences in gene numbers. In order to overcome this problem and to make an equal comparison between these different data sets, orthologs are used within these comparative analyses performed in this study. Orthologs are genes in different species that evolved from a common ancestral gene by speciation. Normally, orthologs retain the same function in the course of evolution. Therefore, in order to compare genes conserved across the two species, the Orthologene Library in Array'Track ${ }^{\mathrm{TM}} 35$ (version 3.4.5., U.S. Food and Drug Administration, Silver Spring, USA) was used for obtaining Homologene IDs for the human and mouse arrays. The content of the Orthologene Library is mainly composed of data from the NCBI HomoloGene database. A list of 7344 Homologene IDs could be obtained (combined with human and mouse EntrezGene ID), from which the human EntrezGene IDs were used for further analyses. ArrayTrack allows rapid matching of a large number of genes across human, mouse and rat for the gene orthology analysis. The coverage of orthologs between human and mouse represented $62 \%$, $56 \%$ and $67 \%$ of the measurable genes, in PMH, HepG 2 and mESC, respectively. These numbers of retained orthologous genes were consistent with previous studies ${ }^{36}$. In the remainder part of this manuscript the word gene lists will refer to the orthologous gene list.

\section{Hierarchical Clustering}

ArrayTrack ${ }^{\mathrm{TM}}$ was used for hierarchical clustering analysis (HCA) of the tested models and time points by using the list of 7344 orthologue genes. For HCA a two-way method is applied 
to investigate the grouping of samples in terms of their similarities in gene expression profiles, as well as the grouping of genes in terms of their similarities of samples. A HCA was produced using Pearson correlation, Euclidean distance with pair wise complete distance in GenePattern v.3.2.1 (http://genepattern.broadinstitute.org).

\section{Gene-based, pathway maps and transcription factor analysis}

To investigate the mutual gene expression changes on the single gene level, individual genes were compared between the DEG lists from all three cell models. To gain an initial insight into the function of the overlapping genes, they were categorized using the KEGG database subcategories. To further map and explore the transcriptional changes across in vitro models and to understand their biological significance into more depth, the DEG lists were further examined with MetaCore ${ }^{\mathrm{TM}}$ (GeneGo, San Diego, USA). MetaCore ${ }^{\mathrm{TM}}$ is an integrated knowledge database and software suite for pathway analysis of experimental data and gene lists. MetaCore ${ }^{\mathrm{TM}}$ is based on a proprietary manually curated database of human proteinprotein, protein-DNA and protein compound interactions, metabolic and signaling pathways for human, mouse and rat, supported by proprietary ontologies and controlled vocabulary. Functional enrichment analysis was used to identify significantly modulated pathway maps. The program scores and ranks the most relevant pathway maps, which can be categorized into cellular processes. Each cellular process category contains pathway maps related to the same biological process, such as e.g. Apoptosis and Survival. In the analysis, the filtered data sets were used as background list (7344 genes). Pathway maps with a $p$ value $<0.05$ and the involvement of at least 2 genes that were significantly modulated ${ }^{37}$. In Metacore ${ }^{\mathrm{TM}}$ both large and small pathways are annotated, the smallest consisting of indeed no more than 2 genes. Where pathways are supposed to describe interactions between genes, it stands to reason that minimally 2 genes are required. In the analysis a background list of the 7344 filtered genes was used. For the overlapping pathways a common p-value was calculated.

In order to explore the complexity of the transcriptional regulatory networks also transcription factor networks were studied. The Transcription Factor Target Modeling workflow in Metacore $^{\mathrm{TM}}$ was used to explore the role of transcription factors in the different gene sets. This workflow generates networks using the Analyze network (transcription factor) algorithm. The algorithm looks for shortest paths from transcription factors to genes. The TF networks are based on literature stored within Metacore and the direct connections between genes and TF are validated with experiments which makes them thrustworthy (see Metacore Manual for more information

https://portal.genego.com/help/MetaCore_Advanced_Training_Manual_5.0.pdf).

Transcription factor networks with a $p$ value $<0.05$ and the involvement of at least 5 seed nodes were regarded as significantly involved. Per model a TF network analysis was performed which resulted in a number of genes associated with each TF. Per model it is then possible to extract all genes associated with these TFs. This was done for a limited set of TFs. These TFassociated genes were then compared with the total number of DEG. The genes that are related to the respective transcription factors were extracted and used for an additional pathway map analysis, using the total list of DEG as a background list. Also these pathway maps were divided over several cellular process categories. 


\section{$\underline{\text { Results }}$}

\section{Effects of cisplatin at the gene level}

Modulation of gene expression profiles by cisplatin in three cell models was determined by use of DNA microarrays. Only genes measurable in all three cell models and for which humanmouse orthologues are described were taken into account. To obtain insight into the comparability of the gene expression profiles from the different models, time points and biological replicates, first a hierarchical clustering analysis (HCA) was performed (Supplementary Figure 2.2). The clustering dendogram shows a distinction between the three models although the two liver models, PMH and HepG2, cluster together. Furthermore the dendogram also depicts a clustering of the biological replicates.

Identification of the DEG revealed that in PMH the highest number was modulated (1987 and 2448 at $24 \mathrm{~h}$ and $48 \mathrm{~h}$, respectively) (Table 2.1). HepG2 cells mainly showed a high response at $24 \mathrm{~h}$ and $48 \mathrm{~h}$ (898 and 1423 , respectively). In mESC, however, the responses were smallest for all time points, with few DEGs at 2-4h and more at $8 \mathrm{~h}$ and $24 \mathrm{~h}$ (3, 5, 53 and 184, respectively). The overlap in responses between two models or between time points within a particular model is summarized in Table 2.1. The overlap in DEGs between time points within a cell model and between the models is relatively high. The largest overlap is observed when comparing the $24 \mathrm{~h}$ and $48 \mathrm{~h}$ incubation periods; less overlap is seen when analysing earlier time points. Furthermore, the overlap between all three models improves when comparing more closely related time points rather than more distant ones. For HepG2 cells and PMH a large part of DEG are unique for that model, indicating that cell type-specific modulations are considerable. The mESC have more DEG in common with PMH than with HepG2 cells, obviously because both are mouse in origin.

Table 2.1 Matrix representing number of DEGa and overlaps between exposure periods and/or cell models

\begin{tabular}{|c|c|c|c|c|c|c|c|c|c|c|c|}
\hline \multirow{3}{*}{ PMH } & & & \multirow{2}{*}{\multicolumn{2}{|c|}{$\begin{array}{r}\text { PMH } \\
\\
48 \mathrm{~h}\end{array}$}} & \multicolumn{2}{|c|}{ HepG2 } & \multicolumn{5}{|c|}{ mESC } \\
\hline & \multicolumn{2}{|c|}{ Total \# DEG } & & & $12 \mathrm{~h}$ & $24 \mathrm{~h}$ & $48 \mathrm{~h}$ & $2 \mathrm{~h}$ & $4 h$ & $8 h$ & $24 \mathrm{~h}$ \\
\hline & 1987 & $24 \mathrm{~h}$ & & 1543 & 170 & 402 & 438 & 0 & 3 & 29 & 92 \\
\hline & 2448 & $48 \mathrm{~h}$ & 1543 & & 177 & 531 & 505 & 0 & 3 & 29 & 107 \\
\hline \multirow[t]{3}{*}{ HepG2 } & 268 & $12 \mathrm{~h}$ & 170 & 177 & & 240 & 143 & 0 & 4 & 9 & 18 \\
\hline & 898 & $24 \mathrm{~h}$ & 402 & 531 & 240 & & 531 & 0 & 4 & 16 & 49 \\
\hline & 1423 & $48 h$ & 438 & 505 & 143 & 531 & & 0 & 3 & 17 & 60 \\
\hline \multirow[t]{4}{*}{ mESC } & 3 & $2 \mathrm{~h}$ & 0 & 0 & 0 & 0 & 0 & & 0 & 0 & 0 \\
\hline & 5 & $4 h$ & 3 & 3 & 4 & 4 & 3 & 0 & & 3 & 1 \\
\hline & 53 & $8 h$ & 29 & 29 & 9 & 16 & 17 & 0 & 3 & & 19 \\
\hline & 183 & $24 \mathrm{~h}$ & 92 & 107 & 18 & 49 & 60 & 0 & 1 & 19 & \\
\hline
\end{tabular}

a significance is based on an absolute $\mathrm{FC}$ of 1.5 and $p$ value $<0.05$ 
Based on the limited number of gene expression changes after short incubation periods, gene expression modifications after incubation periods of the $24 \mathrm{~h}$ and $48 \mathrm{~h}$ for HepG2 and PMH and $24 \mathrm{~h}$ for mESC, were chosen for in depth comparisons. Since mESC are rapidly replicating compared to HepG2 and PMH (the latter model does not replicate at all), we assumed that gene expression changes in HepG2 and PMH might be slower. Indeed, this agrees with the larger overlap of the $48 \mathrm{~h}$ data than of the $24 \mathrm{~h}$ data with the $24 \mathrm{~h}$ mESC data (Table 2.1). Therefore, we combined the gene expression data of both exposure periods for the liver models and compared these with the $24 \mathrm{~h}$ gene expression data of mESC. When analysing the DEG modulated by all models, 52 are in common between the three models (part A of Figure 2.1). For 44 of these the change was in the same direction for all 3 cell models. The expression changes (presented by a heatmap) and functional annotations of the 52 common genes are provided in part B of Figure 2.1. Examples of DEG found at both time points are MDM2, PHLDA3, PPM1D, BTG2 and PERP, which are important in the regulation of apoptosis, cellcycle, cell growth and death and DNA repair. All these KEGG subcategories are related to toxic responsive processes which are frequently affected after a genotoxic insult. Other overlapping genes are involved in the KEGG subcategories of cell signaling or metabolism.

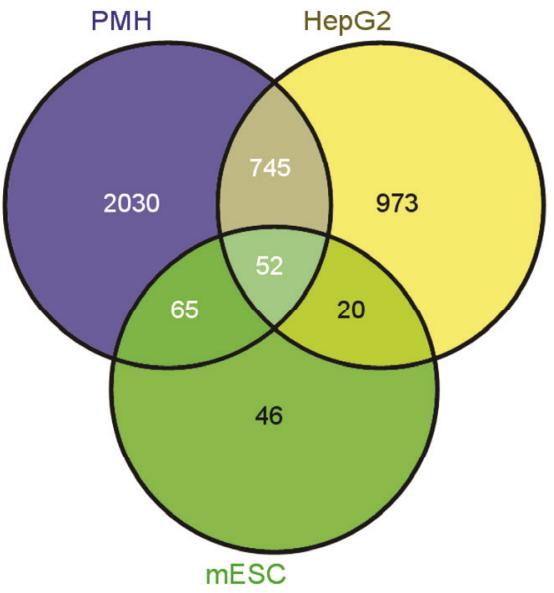

A.
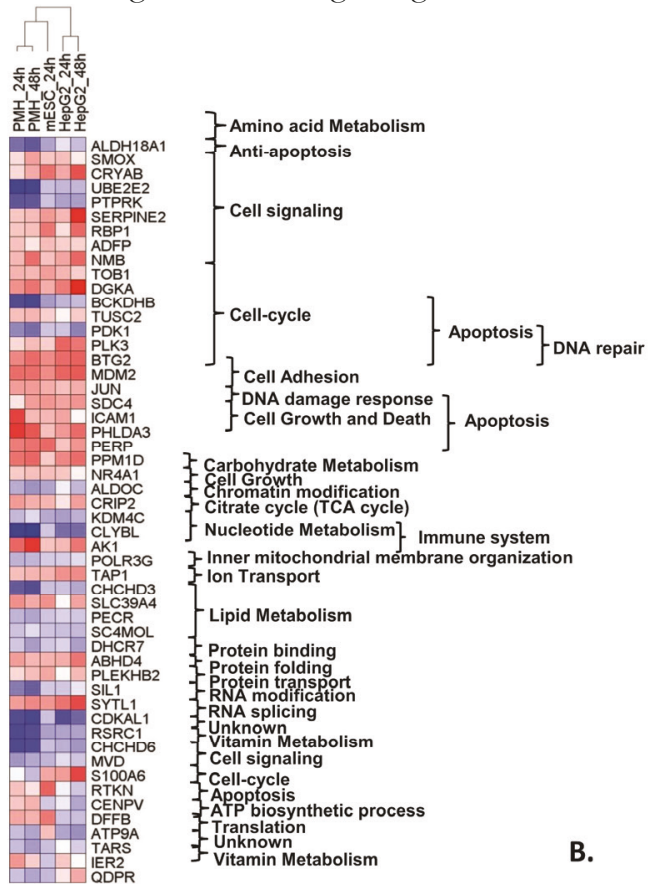

Figure 2.1: (A) The venn diagram depicts the 52 common genes regulated by all models after exposure to cisplatin and (B) the heat map shows the expression changes and functional annotations of the 52 common genes.

\section{Effects on the 44 conserved genes by other GTX compounds}

In order to further investigate whether the conservation of these found genes stretches beyond cisplatin and could also be valid for other genotoxic compounds, expression patterns of these 44 conserved genes were compared with data from our own group on HepG2 and PMH. For these cell models, data for several of the same GTX agents were available. With transcriptomic results from another study in HepG2 cells ${ }^{8}$, we observed a good correlation between the expression changes after cisplatin exposure and the expression changes following exposure to 
several other GTX compounds (e.g. aflatoxin B1, benzo[a]pyrene, chlorambuciland and 1ethyl-1-nitrosourea) (Table 2.2). Additionally expression data from this set of conserved genes were also compared with available data on PMH exposed to a number of GTX compounds (Lizarraga et al., unpublished), which showed an even stronger correlation (aflatoxin B1, benzo(a)pyrene, Diethylnitrosamine, 7,12-Dimethyl benzantracene and Mitomycine C) (Table 2.2). To further support the hypothesis that these conserved genes are also important in the human situation we compared the average expression values from these genes with data available from primary human hepatocytes $(\mathrm{PHH})$ extracted from a large data base (TGGATEs, http://toxico.nibio.go.jp/). From the GTX compounds used in HepG2 and PMH only data on PHH treated with aflatoxin B1 and benzo[a]pyrene were present in this data base. Expression patterns of PHH exposed to aflatoxin B1 showed a good correlation with our set of conserved genes as presented in Table 2.2. Many correlations are very high which might indicate the importance of these genes within the genotoxic response in other models, especially the human models.

Table 2.2: Correlation coefficients of gene expression $\log 2$ ratios of the conserved set of 44 genes in comparison with $\log 2$ ratios from Magkoufopoulou et al. in HepG2 cells, Lizarraga et al. in PMH and TG-GATEs in PHH following exposure to several GTX compounds as calculated with Microsoft Excel (N/A = not available)

\begin{tabular}{|l|l|l|l|l|l|l|}
\hline \multicolumn{2}{|l|}{} & \multicolumn{2}{l}{ HepG2 } & \multicolumn{2}{l|}{ PMH } & PHH \\
\hline Compound & Abbreviation & $24 \boldsymbol{h}$ & $\mathbf{4 8 h}$ & $\mathbf{2 4 h}$ & $\mathbf{4 8 h}$ & $\mathbf{2 4 h}$ \\
\hline Aflatoxin B1 & AFB1 & 0.78 & 0.82 & 0.82 & 0.82 & 0.82 \\
\hline Benzo[a]pyrene & BaP & 0.81 & 0.81 & 0.77 & 0.77 & N/A \\
\hline Diethylnitrosamine & DEN & 0.07 & -0.21 & 0.84 & 0.84 & 0.57 \\
\hline 7,12-Dimethyl benzantracene & DMBA & 0.35 & 0.11 & 0.77 & 0.77 & N/A \\
\hline Mitomycine C & MMC & 0.37 & 0.47 & 0.90 & 0.90 & N/A \\
\hline Cisplatin & cisPt & 0.92 & 0.91 & N/A & N/A & N/A \\
\hline 1-Ethyl-1-nitrosourea & ENU & 0.76 & 0.65 & N/A & N/A & N/A \\
\hline Chlorambucil & Cb & 0.68 & 0.79 & N/A & N/A & N/A \\
\hline
\end{tabular}

\section{Pathway map analysis}

With functional enrichment analysis using Metacore ${ }^{\mathrm{TM}}$ significantly modulated pathway maps were identified for each model for the $24 \mathrm{~h}$ and $48 \mathrm{~h}$ exposure periods, using all DEG per separate model (Supplementary Table 2.1). Pathway maps in which more than 2 genes in total were involved and of which the $p$ value was $<0.05$ were considered significant. For this approach pathway map results from $24 \mathrm{~h}$ and $48 \mathrm{~h}$ were combined. Each significant pathway map was categorized according to the cellular process to which it relates. Pathways related to "Aminoacid metabolism and its regulation_Glycine, serine, cysteine and threonine metabolism" and "Cholesterol and bile acid homeostasis_Cholesterol Biosynthesis" were identified as being present in all models as can be seen in Supplementary Table 2.1. Figure 2.2 presents a Venn diagram with the overlapping and unique pathways between the three models. For the limited set of overlapping pathways a common p-value has been calculated. 


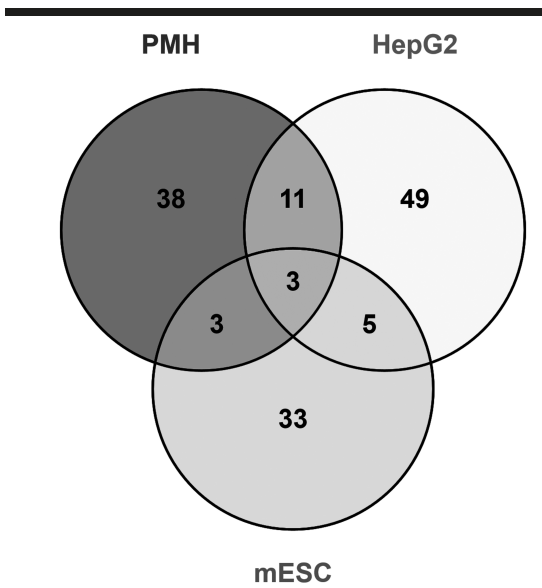

Figure 2.2: The venn diagram depicts the overlapping and unique pathways between the three models when comparing profiles at 24 and $48 \mathrm{~h}$.

\section{Transcription factor analysis}

Finally, transcription factor analysis was performed to investigate whether comparable transcription regulation networks are modulated. With the transcription factor enrichment analysis tool of MetaCore ${ }^{\mathrm{TM}}$ we found $20 \mathrm{TFs}$ per cell model per time point that could potentially regulate the DEG. When comparing the expression of these transcription factor networks between the cell models upon cisplatin challenge, the same 12 TFs were observed for all models, irrespective of exposure time, $24 \mathrm{~h}$ or $48 \mathrm{~h}$ (see Figure 2.3; Supplementary Table 2.2). For the two liver models, additionally AP-1, HNF1- $\alpha$, E2F1, C/EBP- $\beta$ and GCR- $\alpha$ were found, both at $24 \mathrm{~h}$ as well as $48 \mathrm{~h}$, thus these TFs appear tissue-specific. Since we were mainly interested in the conserved response across models we focussed on the top four of TFs that were most enriched among the modulated genes in all models and at all exposure periods, as represented by the largest number of connections (nodes) within one TF network. These were HNF4-alpha, SP-1, c-MYC and p53 (see Supplementary Table 2.2). These four TFs together potentially regulate 911 genes (46\% of the total \# of DEG) in $\mathrm{PMH}, 435$ genes $(48 \%)$ in HepG2 cells and 103 genes (56\%) in mESC after 24h exposure. After 48h of exposure 1107 genes $(45 \%)$ in PMH and 710 genes $(50 \%)$ in HepG2 cells were potentially regulated by these four TFs.

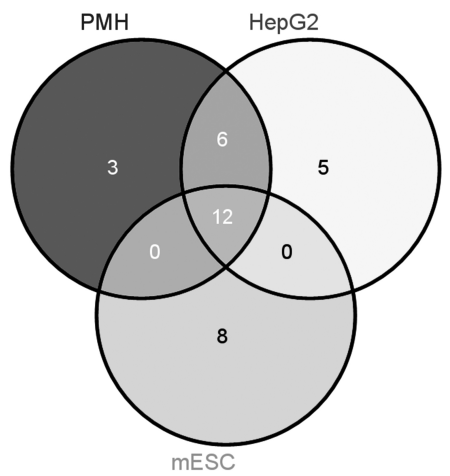

12 Common TF
•HNF4-alpha
-SP1
•c-Myc
-p53
-CREB1
-ESR1 (nuclear)
-NF-kB
-EGR1
-Androgen receptor
•HIF1A
- GATA-1
-ETS1

Figure 2.3: The venn diagram depicts the 12 overlapping conserved TFs (mentioned on right side of the figure) and unique TFs between the three cell models when comparing profiles at 24 and $48 \mathrm{~h}$. 
In order to investigate the processes to be controlled by these TF networks, pathway analysis of the genes regulated by the top four TF for the three liver models was performed. The obtained pathway maps were assigned to several cellular process categories and then compared between the models. These cellular process categories revealed a slightly stronger overlap (see Table 2.3) than when using all DEG for this categorization.

Table 2.3: Overlapping cellular processes and significantly modulated pathway maps by TF-regulated (c-Myc, HNF4- $\alpha$, p53, SP1) genes between the models and at all exposure periods

\begin{tabular}{|c|c|c|c|c|c|}
\hline \multirow[t]{2}{*}{$\begin{array}{l}\text { Cellular } \\
\text { process }\end{array}$} & \multirow[t]{2}{*}{$\begin{array}{l}\text { Modulated pathway } \\
\text { maps }\end{array}$} & \multirow[t]{2}{*}{ Cell modela } & \multirow[t]{2}{*}{ Contributing TF } & \multicolumn{2}{|c|}{$\begin{array}{l}\text { Genes in pathway } \\
\text { regulated by specific TF }\end{array}$} \\
\hline & & & & Significant & Total b \\
\hline \multirow[t]{2}{*}{$\begin{array}{l}\text { Growth and } \\
\text { differentiation }\end{array}$} & $\begin{array}{l}\text { Glucocorticoid } \\
\text { receptor signaling }\end{array}$ & $\begin{array}{l}\text { A } \\
\text { B } \\
\text { C } \\
\text { D } \\
\text { E }\end{array}$ & $\begin{array}{l}\text { c-Myc/HNF4- } \alpha \\
\text { HNF4- } \alpha \\
\text { c-Myc/HNF4- } \alpha / \text { p } 53 / \text { SP-1 } \\
\text { c-Myc/HNF4- } \alpha / \text { p53/SP-1 } \\
\text { c-Myc }\end{array}$ & $\begin{array}{l}4 / 4 \\
4 \\
4 / 4 / 4 / 5 \\
5 / 6 / 4 / 6 \\
4\end{array}$ & $\begin{array}{l}4 \\
4 \\
5 \\
6 \\
4\end{array}$ \\
\hline & $\begin{array}{l}\text { Thrombopoietin- } \\
\text { regulated cell } \\
\text { processes }\end{array}$ & $\begin{array}{l}\text { A } \\
B \\
C \\
D \\
\text { E }\end{array}$ & $\begin{array}{l}\text { HNF4- } \alpha / \text { p53/SP-1 } \\
\text { p53/SP-1 } \\
\text { p53/SP-1 } \\
\text { p53 } \\
\text { HNF4- } \alpha / \text { p53 }\end{array}$ & $\begin{array}{l}7 / 6 / 9 \\
6 / 8 \\
4 / 4 \\
5 \\
3 / 3\end{array}$ & $\begin{array}{l}10 \\
9 \\
4 \\
6 \\
3\end{array}$ \\
\hline \multirow[t]{2}{*}{ Cell cycle } & $\begin{array}{l}\text { ESR1 regulation of } \\
\text { G1/S transition }\end{array}$ & $\begin{array}{l}\text { A } \\
\text { B } \\
\text { C } \\
\text { D } \\
\text { E }\end{array}$ & $\begin{array}{l}\text { c-Myc/HNF4- } \alpha / \text { p53/SP-1 } \\
\text { c-Myc/p53/SP-1 } \\
\text { c-Myc/HNF4- } \alpha / \text { p53/SP-1 } \\
\text { c-Myc/HNF4- } \alpha / \text { SP-1 } \\
\text { HNF4- } \alpha / \text { p53 }\end{array}$ & $\begin{array}{l}5 / 4 / 3 / 5 \\
4 / 3 / 4 \\
5 / 4 / 4 / 5 \\
7 / 7 / 6 \\
3 / 3\end{array}$ & $\begin{array}{l}5 \\
4 \\
5 \\
7 \\
3\end{array}$ \\
\hline & $\begin{array}{l}\text { Influence of Ras and } \\
\text { Rho proteins on } \\
\text { G1/S Transition }\end{array}$ & $\begin{array}{l}\text { A } \\
\text { B } \\
\text { C } \\
\text { D } \\
\text { E }\end{array}$ & $\begin{array}{l}\text { HNF4- } \alpha / \mathrm{p} 53 / \mathrm{SP}-1 \text { p53/ } \\
\text { SP-1 } \\
\text { c-Myc/HNF4- } \alpha / \text { p } 53 / \text { SP-1 } \\
\text { c-Myc/HNF4- } \alpha / \text { p } 53 / \text { SP-1 } \\
\text { HNF4- } \alpha / \text { p } 53\end{array}$ & $\begin{array}{l}6 / 5 / 8 \\
7 / 8 \\
7 / 7 / 8 / 10 \\
7 / 6 / 7 / 7 \\
3 / 3\end{array}$ & $\begin{array}{l}10 \\
9 \\
10 \\
9 \\
3\end{array}$ \\
\hline \multirow[t]{2}{*}{ Transcription } & $\begin{array}{l}\text { P53 signaling } \\
\text { pathway }\end{array}$ & $\begin{array}{l}\text { A } \\
\text { B } \\
\text { C } \\
\text { D } \\
\text { E }\end{array}$ & $\begin{array}{l}\text { c-Myc/HNF4- } \alpha / \mathrm{p} 53 \\
\mathrm{c}-\mathrm{Myc} / \mathrm{p} 53 \\
\text { p } 53 \\
\text { p } 53 / \mathrm{SP}-1 \\
\mathrm{HNF} 4-\alpha / \mathrm{p} 53\end{array}$ & $\begin{array}{l}7 / 6 / 8 \\
5 / 7 \\
5 \\
4 / 4 \\
3 / 3 \\
\end{array}$ & $\begin{array}{l}9 \\
8 \\
5 \\
4 \\
3\end{array}$ \\
\hline & $\begin{array}{l}\text { Ligand-dependent } \\
\text { activation of the } \\
\text { ESR1/SP pathway }\end{array}$ & $\begin{array}{l}\text { A } \\
\text { B } \\
\text { C } \\
\text { D } \\
\text { E }\end{array}$ & $\begin{array}{l}\text { p53 } \\
\text { p53 } \\
\text { HNF4- } \alpha / \text { p53 } \\
\text { c-Myc/HNF4- } \alpha \\
\text { HNF4- } \alpha / \text { p53 }\end{array}$ & $\begin{array}{l}7 / 6 / 5 / 7 \\
4 \\
3 / 3 \\
4 / 5 \\
3 / 3\end{array}$ & $\begin{array}{l}9 \\
7 \\
3 \\
5 \\
3\end{array}$ \\
\hline Proteolysis & $\begin{array}{l}\text { Putative SUMO-1 } \\
\text { pathway }\end{array}$ & $\begin{array}{l}\text { A } \\
\text { B } \\
\text { C } \\
\text { D } \\
\text { E }\end{array}$ & $\begin{array}{l}\mathrm{p} 53 / \mathrm{SP}-1 \\
\mathrm{p} 53 / \mathrm{SP}-1 \\
\mathrm{p} 53 \\
\mathrm{p} 53 / \mathrm{SP}-1 \\
\mathrm{HNF} 4-\alpha / \mathrm{p} 53\end{array}$ & $\begin{array}{l}5 / 6 \\
6 / 7 \\
3 \\
4 / 4 \\
3 / 3 \\
\end{array}$ & $\begin{array}{l}7 \\
8 \\
3 \\
4 \\
3\end{array}$ \\
\hline
\end{tabular}

a A = PMH 24h; B = PMH 48h; C = HepG2 24h; D = HepG2 48h; E = mESC 24h.

$\mathrm{b}$ Total number of genes in background list represented by all DEG.

The cellular process categories that were significantly modulated were involved in "Cell growth and differentiation", "Cell cycle", "Transcription" and "Proteolysis" (4). In many of these pathways a number of the same genes appear to be involved, such as Ak1, PKC, GCR-alpha, c-Jun, c-Fos and some MAPKs (such as ERK, JNK, and p38 MAPK). The "p53 signalling pathway map" and the "Ligand-dependent activation of the ESR1/SP pathway map", both belonging to the cellular process category of "Transcription" (presented in Supplementary Figure 2.3) visualizes the differences and the similarities between the three cell models with respect to p53 and SP1. 
Additionally the interplay between these crucial TFs in the conserved cisplatin response is further supported with a network (presented in Figure 2.4) depicting the regulation of the set of 44 conserved genes found within this study by HNF4-alpha, SP-1, c-MYC and p53.

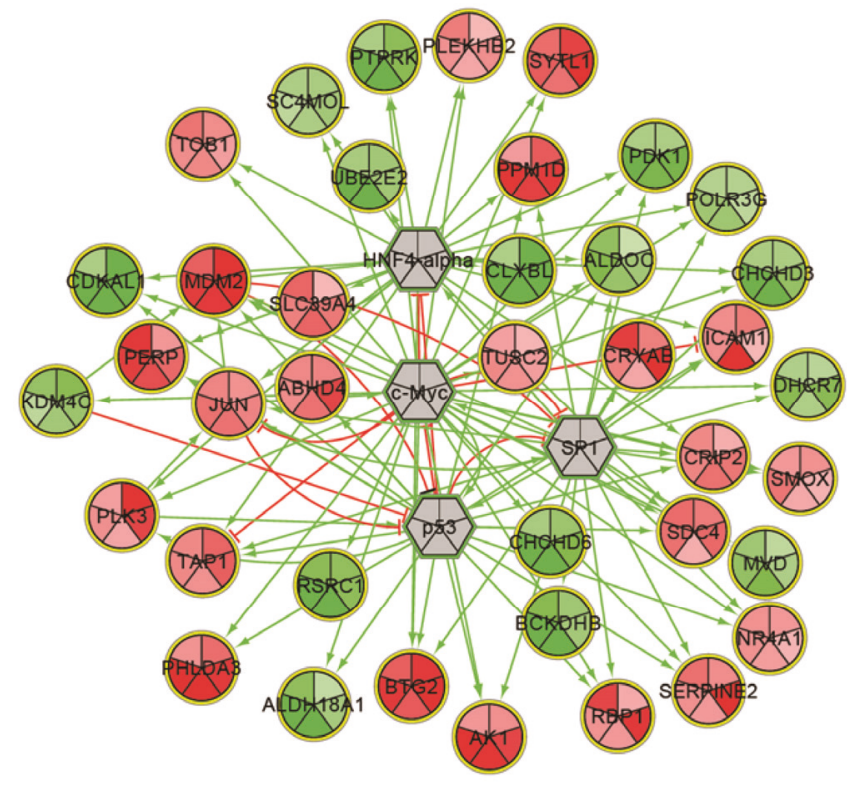

Figure 2.4: Forty-four conserved genes regulated by top four of most important TFs: SP1, p53, c-Myc and HNF4-alpha. Green and red edges between nodes mean stimulation and inhibition, respectively. Ellipse (genes) and hexagon (TFs). Gene expression values are depicted through the green and red nodes (between -4 and +4$)$ in a clockwise direction (HepG2 24h $\rightarrow$ HepG2 48h $\rightarrow$ PMH 24h $\rightarrow$ PMH 48h $\rightarrow$ mESC 24h).

\section{Discussion}

In this study cisplatin-induced transcriptomic profiles of PMH, HepG2 cells and mESC were investigated to gain insight into the conserved cross in vitro model responses to a direct acting DNA damaging agent. By using orthologous genes, the transcriptomic responses of all models were compared on three levels: gene, pathway maps and TF networks.

The 44 conserved genes, altered in the same direction in all three models, are involved in processes such as apoptosis, cell cycle, cell growth and death and DNA repair. Some of these genes have already been reported in the paper from Kruse et al. in mESC. Furthermore, according to the Comparative Toxicogenomics Database (CTD), MDM2 ${ }^{38}$, SERPINE2, BTG2 ${ }^{39}$ and TAP1 ${ }^{40}$ have been associated before with cisplatin exposure in other cell models. Some of the other genes found in this study have also been shown to be affected after exposure to other GTX chemicals such as for example benzo(a)pyrene and aflatoxin B1 in $\mathrm{PMH}{ }^{41}$ or diethylnitrosamine in mice ${ }^{42}$. Moreover when comparing the expression patterns of these conserved genes, found after cisplatin exposure, with data from other studies in HepG2 and PMH, exposed to several GTX compounds, we find a strong correlation thereby indicating the importance of these genes within the conserved response to a GTX insult. The relevance of these genes for the human situation is further supported by the fact that the expression values of these conserved genes found in this study also show a good correlation to data from PHH exposed to at least another GTX compound. From the GTX compounds used 
in HepG2 and PMH, unfortunately only data on PHH treated with aflatoxin B1 and benzo[a]pyrene were present in this data base. However, by our approach we were able to identify a set of genes with conserved transcriptomics responses in several mouse and human cell models, indicating that these genes are an important part of the conserved GTX response in mammalian cells. These results further substantiate that the models used are robust since the conserved genes show promising correlation with other GTX compounds and other in vitro models.

Functional analyses in MetaCore ${ }^{\mathrm{TM}}$ were performed for each model separately compared between the models. This revealed to a small extent common effects related to "Aminoacid metabolism and its regulation_Glycine, serine, cysteine and threonine metabolism" and "Cholesterol and bile acid homeostasis_Cholesterol Biosynthesis", thus indicating a contribution of regulated metabolism-related pathways or processes, which seem to be downregulated by cisplatin. These findings are in line with a previous study performed in rat hepatocytes in which both on the genomic as well as the proteomic level these processes seemed to be affected by cisplatin exposure ${ }^{43}$. Remarkable, we were not able to detect any effect of cisplatin exposure on common significant pathway maps related with DNA damage response. This agrees with a study in which the phosphoproteome, the proteome as well as the transcriptome were analyzed in mESC after cisplatin exposure. That study suggested that the impact of cisplatin on the DNA damage response pathways was only manifested in the phosphoproteome analysis, indicating that phosphorylation events are key in activating DNA response pathways after GTX stress induced by cisplatin ${ }^{18}$.

Changes in TF regulatory networks were also studied, as they are thought to be conserved during evolution 27 and have been shown before to play an important role in the transcriptomic response of HepG2 cells to exposure to the GTX compound benzo[a]pyrene ${ }^{17}$. Comparisons based on TF networks revealed a higher degree of conservation between the models than based on analyses on gene or pathway map level. Twelve TFs were overall found to be connected to the different sets of DEG for the three models. The top four of these TFs (c-MYC, HNF4- $\alpha$, p53 and SP1) are hypothesized to be responsible for up to $50 \%$ of the DEG in the different models. Three of these TFs, namely c-MYC, p53 and SP1 have been described before in literature to be involved in the cellular response to cisplatin exposure ${ }^{44}$. HNF4-alpha and SP1 have also been reported to play a role within the classification of compounds based on their GTX or NGTX properties using a large set of chemicals using HepG2 cells as a model ${ }^{8}$. In order to shed light on the possible functions of these TFs, pathway map analysis was subsequently performed with a subset of these TF regulated DEG per model separately. This indicated a strong overlap in cellular processes within these three cell models, such as cellular processes related to "Glucocorticoid receptor signalling", "Thrombopoietin-regulated cell processes ", "ESR1 regulation of G1/S transition", "Influence of Ras and Rho proteins on G1/S Transition", "P53 signaling pathway", "Ligand-dependent activation of the ESR1/SP pathway" and "Thrombopoietin-regulated cell processes". An overlapping subset of DEG within these categories could be observed between the models to be regulated by the same TF. However, also differences between the models remain. This indicates that the precise regulation of DEG by the same TFs can be conserved as well as cell model specific and therefore suggest that a complex network of a conserved subset of TFs is responsible for the changes in genes after cisplatin exposure. In agreement with this, several genome-wide transcription factor binding studies have shown that the patterns of some transcription factors can depend considerably on cell type, stage of the cell cycle or environmental conditions ${ }^{45}$.

This cell type and differentiation state specific role of for example p53 is illustrated in the visualization of the "p53 signaling pathway map" (depicted in Supplementary Figure 2.3). In this pathway map both a subset of conserved DEG can be observed as well as cell-model 
specific DEG, which together appear responsible for the ultimate net effect for this pathway map. Here it can also be seen that the key regulators like p53 and ATM are not significantly changed in their gene expression in the in vitro models, which is in concordance with the study in mESC that showed that the DNA damage response is mainly affected at the phosphoproteome level ${ }^{18}$. Genes that are important in DNA damage response and actually are under control by these key regulators, such as MDM2 and p21, are indeed affected on the gene expression level in all models and at both time points. Increased levels of MDM2 would inactivate the apoptotic and cell cycle arrest functions of p53 ${ }^{46}$. Besides reducing p53 levels, MDM2 also promotes the expression of $\mathrm{p} 21$ and $\mathrm{Rb}$ protein, which are important regulators of cell cycle and cell death. Furthermore MDM2 is also able to bind to SP1 and inhibits SP1dependent transcription ${ }^{47}$. SP1 is a TF that influences transcription in response to physiological and pathological stimuli and regulates the expression of a large number of genes involved in a variety of processes such as cell growth, apoptosis, differentiation and immune responses. In addition it is highly regulated by post-translational modifications, may also have a role in modulating the cellular response to DNA damage and is implicated in chromatin remodelling ${ }^{48}$. Since SP1 could possibly play a crucial role in these pivotal processes in all cell models used, it seems likely that this particular TF is found to be linked with this specific conserved response.

Another TF found to be important within the conserved response is HNF4- $\alpha$, which plays an essential role in the development and function organs, including hepatocytes by regulating expression of multiple genes involved in organ development, nutrient transport, and diverse metabolic pathways (e.g. cholesterol, fatty acid and glucose metabolism but also xenobiotic and drug metabolism) ${ }^{49}$. Furthermore HNF4- $\alpha$ is highly expressed in human hepatocytes ${ }^{50}$ as well as HepG2 cells ${ }^{51}$ and plays a role in differentiation and functioning of the cell. In addition to its role in regulating genes responsible for differentiation and development, there is also an increasing amount of evidence suggesting that $\mathrm{HNF} 4 \alpha$ might also play a role in regulating the cell cycle. Therefore, it seems likely that especially in the two liver models, but also in the mESC, this TF is crucial in certain processes as can be seen in Table 2.3. Both SP1 as HNF4- $\alpha$ both have been shown to be able to bind to promoter sites of genes involved in cell proliferation in kidney and tumor cells respectively ${ }^{52,53}$. Furthermore SP1 seems to be regulated by p53 through multiple mechanisms ${ }^{53}$. Moreover, in a study with HepG2 cells ${ }^{54}$, overexpressed HNF4- $\alpha$ was found to activate the expression of p21 primarily by interacting with promoter-bound SP1.

Furthermore, MYC (c-MYC), is a TF that promotes cell growth and proliferation, and, under certain conditions, apoptosis. Additionally it has shown to be involved in stem cell renewal 55 . Therefore it is highly expressed in embryonic stem cells, but also in human tumors. MYC is a strong proto-oncogene and it is often found to be upregulated in many types of cancers. Levels of MYC are increased or decreased in response to mitogenic or growth-inhibitory stimuli, respectively. In addition MYC has been shown to be a principal determinant in the crucial determination process of a cell to either undergo cell cycle arrest or apoptosis to cope with DNA damage in colon cancer cells ${ }^{56}$. MYC can therefore block p21 induction by p53 and thereby prevent transcriptional activation of this important regulator of cell cycle. The cell can then change from a cytostatic to an apoptotic fate. A study performed in HepG2 cells ${ }^{54}$ also observed an interaction between HNF4 $\alpha 1$ and c-MYC as well as a competition between these two transcription factors for interaction with promoter-bound SP1 and regulation of $\mathrm{p} 21$. These two TF may therefore compete for control of genes involved in cell proliferation and differentiation, which can also be hypothesized from this recent study. c-MYC was also identified as an important regulator of the transcriptomic response in HepG2 cells to benzo[a]pyrene ${ }^{17}$. Several studies have also proven that c-MYC can regulate and compete 
with a number of different transcription factors, including HNF- $\alpha$, SP1 and p53 ${ }^{57}$. This may imply that all other TF described in our study might be regulated by or compete with c-MYC. When we look at our data we could conclude that, despite the complexity of these TF networks, we observed a common response on TF level supported by the fact that the set of conserved genes found within this study is regulated by these four crucial TFs. These findings indicate that these TFs are important in the conserved response to cisplatin in these in vitro cell models.

To further substantiate these findings at the TF level, studies on for example the protein level, like the ones mentioned before ${ }^{18,43}$, are needed since many TFs need post-translational modulation in order to be activated. RNA interference studies might be interesting to further explore the roles of these TF in the cisplatin induced response. In addition, a recent study suggests that microRNAs (miR-34, 192 and 215) also play an important role in the regulation of the cisplatin-induced DNA damage response ${ }^{58,59}$. C-MYC and p53 have been shown to be involved, together with specific microRNAs, in so called feed forward loops. The interplay between TF and perhaps other post-transcriptional modifications such as microRNAs together with their regulated genes could eventually help to further define and understand the conserved cisplatin-induced genotoxicity responses between species.

More studies are needed to explore the role of these TF together with other transcriptional and post-transcriptional regulators in these type of toxic responses. Another study performed in HepG2 cells ${ }^{8}$ already supports the finding of HNF4-alpha and SP1 to be important in the GTX response. In order to validate the findings within this study it would furthermore be desirable to compare these results with expression values within other relevant in vitro models, such as human embryonic stem cells, following exposure to cisplatin but also in response to other GTX compounds. Furthermore, a connectivity map based approach ${ }^{60}$ might be a good alternative for future analysis, so this needs further attention in follow-up studies.

In summary, we conclude that we have identified transcriptomic responses conserved between hepatic/non-hepatic and differentiated/undifferentiated cell models from human and mouse origin, following exposure to the DNA damage inducing agent cisplatin. We were able to advance beyond our previous work by demonstrating that this this conservation is especially apparent on the TF networks level, but also on that of genes encoding proteins. These networks are complicated and difficult to interpret. However, this study may form the basis for follow-up investigation exploring the role of these TF together with other transcriptional and post-transcriptional regulators in these types of toxic responses.

\section{Supplementary data}

Supplementary Figures 2.1-2.3 and Tables 2.1 and 2.2 are available at Mutagenesis Online. 


\section{References}

1.

Benigni, R. Alternatives to the carcinogenicity bioassay for toxicity prediction: are we there yet? Expert Opinion on Drug Metabolism \&\#x26; Toxicology 8, 407-417 (2012).

Kirkland, D. Improvements in the reliability of in vitro genotoxicity testing. Expert Opin Drug Metab Toxicol 7, 1513-20 (2011).

Ellinger-Ziegelbauer, H., Aubrecht, J., Kleinjans, J.C. \& Ahr, H.J. Application of toxicogenomics to study mechanisms of genotoxicity and carcinogenicity. Toxicol Lett 186, 36-44 (2009).

Davila, J.C., Rodriguez, R.J., Melchert, R.B. \& Acosta, D., Jr. Predictive value of in vitro model systems in toxicology. Annu Rev Pharmacol Toxicol 38, 63-96 (1998).

Castell, J.V., Jover, R., Martinez-Jimenez, C.P. \& Gomez-Lechon, M.J. Hepatocyte cell lines: their use, scope and limitations in drug metabolism studies. Expert Opin Drug Metab Toxicol 2, 183-212 (2006).

Mathijs, K. et al. Assessing the metabolic competence of sandwich-cultured mouse primary hepatocytes. Drug Metab Dispos 37, 1305-11 (2009).

Jennen, D.G. et al. Comparison of HepG2 and HepaRG by whole-genome gene expression analysis for the purpose of chemical hazard identification. Toxicol Sci 115, 66-79 (2010).

Magkoufopoulou, C. et al. A transcriptomics-based in vitro assay for predicting chemical genotoxicity in vivo. Carcinogenesis 33, 1421-9 (2012).

Knasmuller, S. et al. Use of human-derived liver cell lines for the detection of environmental and dietary genotoxicants; current state of knowledge. Toxicology 198, 315-28 (2004).

Knasmuller, S. et al. Use of metabolically competent human hepatoma cells for the detection of mutagens and antimutagens. Mutat Res 402, 185-202 (1998).

Schoonen, W.G., Westerink, W.M. \& Horbach, G.J. High-throughput screening for analysis of in vitro toxicity. EXS 99, 401-52 (2009).

Tsamou, M. et al. Performance of in vitro gammaH2AX assay in HepG2 cells to predict in vivo genotoxicity. Mutagenesis 27, 645-52 (2012).

Uhl, M., Helma, C. \& Knasmuller, S. Evaluation of the single cell gel electrophoresis assay with human hepatoma (Hep G2) cells. Mutat Res 468, 213-25 (2000).

Westerink, W.M., Stevenson, J.C., Horbach, G.J. \& Schoonen, W.G. The development of RAD51C, Cystatin A, p53 and Nrf2 luciferase-reporter assays in metabolically competent HepG2 cells for the assessment of mechanism-based genotoxicity and of oxidative stress in the early research phase of drug development. Mutat Res 696, 21-40 (2010).

Fabre, N. et al. A New Hepatoma Cell Line for Toxicity Testing at Repeated Doses. Cell Biology and Toxicology 19, 71-82 (2003).

Guillouzo, A. et al. The human hepatoma HepaRG cells: a highly differentiated model for studies of liver metabolism and toxicity of xenobiotics. Chem Biol Interact 168, 66-73 (2007).

van Delft, J.H. et al. Time series analysis of benzo[A]pyrene-induced transcriptome changes suggests that a network of transcription factors regulates the effects on functional gene sets. Toxicol Sci 117, 381-92 (2010). Pines, A. et al. Global phosphoproteome profiling reveals unanticipated networks responsive to cisplatin treatment of embryonic stem cells. Mol Cell Biol 31, 4964-77 (2011).

Hendriks, G. et al. The ToxTracker assay: novel GFP reporter systems that provide mechanistic insight into the genotoxic properties of chemicals. Toxicol Sci 125, 285-98 (2012).

Bjerkvig, R., Tysnes, B.B., Aboody, K.S., Najbauer, J. \& Terzis, A.J. Opinion: the origin of the cancer stem cell: current controversies and new insights. Nat Rev Cancer 5, 899-904 (2005).

Davila, J.C. et al. Use and application of stem cells in toxicology. Toxicol Sci 79, 214-23 (2004).

Frazer, K.A., Elnitski, L., Church, D.M., Dubchak, I. \& Hardison, R.C. Cross-species sequence comparisons: a review of methods and available resources. Genome Res 13, 1-12 (2003).

Hall, M.D., Okabe, M., Shen, D.W., Liang, X.J. \& Gottesman, M.M. The role of cellular accumulation in determining sensitivity to platinum-based chemotherapy. Annu Rev Pharmacol Toxicol 48, 495-535 (2008).

Burczynski, M.E. et al. Toxicogenomics-based discrimination of toxic mechanism in HepG2 human hepatoma cells. Toxicol Sci 58, 399-415 (2000).

Galluzzi, L. et al. Molecular mechanisms of cisplatin resistance. Oncogene 31, 1869-83 (2012).

Zicca, A. et al. Reduction of cisplatin hepatotoxicity by procainamide hydrochloride in rats. European journal of pharmacology 442, 265-72 (2002).

Vaquerizas, J.M., Kummerfeld, S.K., Teichmann, S.A. \& Luscombe, N.M. A census of human transcription factors: function, expression and evolution. Nat Rev Genet 10, 252-63 (2009).

Staal, Y.C. et al. Binary PAH mixtures cause additive or antagonistic effects on gene expression but synergistic effects on DNA adduct formation. Carcinogenesis 28, 2632-40 (2007).

Mosmann, T. Rapid colorimetric assay for cellular growth and survival: application to proliferation and cytotoxicity assays. J Immunol Methods 65, 55-63 (1983). 
30. Kruse, J.J. et al. A portrait of cisplatin-induced transcriptional changes in mouse embryonic stem cells reveals a dominant p53-like response. Mutat Res 617, 58-70 (2007).

31. Eijssen, L.M. et al. User-friendly solutions for microarray quality control and pre-processing on ArrayAnalysis.org. Nucleic Acids Res 41, W71-6 (2013).

32. Chen, J., Hsueh, H.-M., Delongchamp, R., Lin, C.-J. \& Tsai, C.-A. Reproducibility of microarray data: a further analysis of microarray quality control (MAQC) data. BMC Bioinformatics 8, 412 (2007).

33. Bourgon, R., Gentleman, R. \& Huber, W. Independent filtering increases detection power for highthroughput experiments. Proceedings of the National Academy of Sciences 107, 9546-9551 (2010).

34. Guo, L. et al. Rat toxicogenomic study reveals analytical consistency across microarray platforms. Nat Biotechnol 24, 1162-9 (2006).

35. Tong, W. et al. ArrayTrack--supporting toxicogenomic research at the U.S. Food and Drug Administration National Center for Toxicological Research. Environ Health Perspect 111, 1819-26 (2003).

36. Liao, B.Y. \& Zhang, J. Evolutionary conservation of expression profiles between human and mouse orthologous genes. Mol Biol Evol 23, 530-40 (2006).

37. Jennen, D. et al. Integrating transcriptomics and metabonomics to unravel modes-of-action of 2,3,7,8tetrachlorodibenzo-p-dioxin (TCDD) in HepG2 cells. BMC Syst Biol 5, 139 (2011).

38. Vaskivuo, L. et al. Azidothymidine and cisplatin increase p14ARF expression in OVCAR-3 ovarian cancer cell line. Toxicol Appl Pharmacol 216, 89-97 (2006).

39. Györffy, B. et al. Gene expression profiling of 30 cancer cell lines predicts resistance towards 11 anticancer drugs at clinically achieved concentrations. International Journal of Cancer 118, 1699-1712 (2006).

40. Gutekunst, M. et al. p53 hypersensitivity is the predominant mechanism of the unique responsiveness of testicular germ cell tumor (TGCT) cells to cisplatin. PLoS ONE 6, e19198 (2011).

41. Mathijs, K. et al. Discrimination for genotoxic and nongenotoxic carcinogens by gene expression profiling in primary mouse hepatocytes improves with exposure time. Toxicol Sci 112, 374-84 (2009).

42. Mayhew, C.N. et al. RB loss abrogates cell cycle control and genome integrity to promote liver tumorigenesis. Gastroenterology 133, 976-984 (2007).

43. Cho, Y.E. et al. In-depth identification of pathways related to cisplatin-induced hepatotoxicity through an integrative method based on an informatics-assisted label-free protein quantitation and microarray gene expression approach. Mol Cell Proteomics 11, M111 010884 (2012).

44. Torigoe, T. et al. Cisplatin resistance and transcription factors. Curr Med Chem Anticancer Agents 5, 15-27 (2005).

45. van Steensel, B. Mapping of genetic and epigenetic regulatory networks using microarrays. Nat Genet 37 Suppl, S18-24 (2005).

46. Meek, D.W. \& Knippschild, U. Posttranslational Modification of MDM2. Molecular Cancer Research 1, $1017-$ 1026 (2003).

47. Zhang, Z. \& Zhang, R. p53-independent activities of MDM2 and their relevance to cancer therapy. Curr Cancer Drug Targets 5, 9-20 (2005).

48. Iwahori, S. et al. Identification of phosphorylation sites on transcription factor Sp1 in response to DNA damage and its accumulation at damaged sites. Cell Signal 20, 1795-803 (2008).

49. Lu, P. et al. Structural basis of natural promoter recognition by a unique nuclear receptor, HNF4alpha. Diabetes gene product. J Biol Chem 283, 33685-97 (2008).

50. Kamiyama, Y. et al. Role of human hepatocyte nuclear factor 4alpha in the expression of drug-metabolizing enzymes and transporters in human hepatocytes assessed by use of small interfering RNA. Drug Metab Pharmacokinet 22, 287-98 (2007).

51. Martinez-Jimenez, C.P., Castell, J.V., Gomez-Lechon, M.J. \& Jover, R. Transcriptional activation of CYP2C9, CYP1A1, and CYP1A2 by hepatocyte nuclear factor 4alpha requires coactivators peroxisomal proliferator activated receptor-gamma coactivator 1 alpha and steroid receptor coactivator 1. Mol Pharmacol 70, 1681-92 (2006).

52. Grigo, K., Wirsing, A., Lucas, B., Klein-Hitpass, L. \& Ryffel, G.U. HNF4 alpha orchestrates a set of 14 genes to down-regulate cell proliferation in kidney cells. Biol Chem 389, 179-87 (2008).

53. Black, A.R., Black, J.D. \& Azizkhan-Clifford, J. Sp1 and kruppel-like factor family of transcription factors in cell growth regulation and cancer. J Cell Physiol 188, 143-60 (2001).

54. Hwang-Verslues, W.W. \& Sladek, F.M. Nuclear receptor hepatocyte nuclear factor 4alpha1 competes with oncoprotein c-Myc for control of the p21/WAF1 promoter. Mol Endocrinol 22, 78-90 (2008).

55. Varlakhanova, N.V. et al. myc maintains embryonic stem cell pluripotency and self-renewal. Differentiation 80, 9-19 (2010).

56. Seoane, J., Le, H.V. \& Massague, J. Myc suppression of the p21(Cip1) Cdk inhibitor influences the outcome of the p53 response to DNA damage. Nature 419, 729-34 (2002).

57. Peng, C.-H. et al. A Novel Molecular Signature Identified by Systems Genetics Approach Predicts Prognosis in Oral Squamous Cell Carcinoma. PLoS ONE 6, e23452 (2011).

58. He, L. et al. A microRNA component of the p53 tumour suppressor network. Nature 447, 1130-4 (2007). 
59. Georges, S.A. et al. Coordinated regulation of cell cycle transcripts by p53-Inducible microRNAs, miR-192 and miR-215. Cancer Res 68, 10105-12 (2008).

60. Caiment, F., Tsamou, M., Jennen, D. \& Kleinjans, J. Assessing compound carcinogenicity in vitro using connectivity mapping. Carcinogenesis 35, 201-7 (2014). 
C)
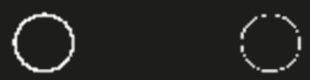

O

○<smiles>[O]</smiles>

$\bigcirc$



Chapter 3

00

() 00

Evaluating microRNA profiles geveals discriminative responses following

genotoxic or non-genotoxic carcinogen exposure in primary mouse hepatocytes?

00

Mutagenesis. 2015 May 14

O

O

0

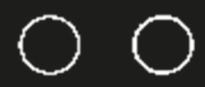

O



0

C)

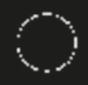

()

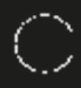

○

0

00

00

(3)

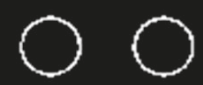

○

$(3)$

Rieswijk L.

Brauers K.J.J.

Coonen M.L.J.

van Breda S.G.J.

Jennen D.6.J.

Kleinjans J.C.S.

0 


\section{$\underline{\text { Abstract }}$}

Chemical carcinogenesis can be induced by genotoxic (GTX) or non-genotoxic (NGTX) carcinogens. GTX carcinogens have a well-described mode of action (MOA). However, the complex mechanisms by which NGTX carcinogens act are less clear and may result in conflicting results between species (e.g. Wy-14,643). We hypothesize that common microRNA response pathways exist for each class of carcinogenic agents. Therefore, this study compares and integrates mRNA and microRNA expression profiles following short term acute exposure (24 and 48h) to three GTX (aflatoxin B1, benzo(a)pyrene and cisplatin) or three NGTX (2,3,7,8-tetrachloordibenzodioxine, cyclosporine $\mathrm{A}$ and $\mathrm{Wy}-14,643)$ carcinogens in primary mouse hepatocytes. Discriminative gene sets, microRNAs (not for $24 \mathrm{~h}$ ) and processes were identified following $24 \mathrm{~h}$ and $48 \mathrm{~h}$ of exposure. From the 3 discriminative microRNAs found following $48 \mathrm{~h}$ of exposure, mmu-miR-503-5p revealed to have an interaction with mRNA target gene cyclin D2 (Ccnd2 - 12444) which was involved in the discriminative process of p53 signaling and metabolism. Following exposure to NGTX carcinogens Mmu-miR-503-5p may have an oncogenic function by stimulating Ccnd2 possibly leading to a tumorigenic cell cycle progression. By contrast, after GTX carcinogen exposure it may have a tumor-suppressive function (repressing $\mathrm{Ccnd} 2$ ) leading to cell cycle arrest and to increased DNA repair activities. In addition, compound-specific microRNA-mRNA interactions (mmu-miR-301b-3p-Papss2 (for aflatoxin B1), as well as mmu-miR-29b-3p-Col4a2 and mmu-miR-24-3p-Flna (for benzo(a)pyrene)) were found to contribute to a better understanding of microRNAs in cell cycle arrest and the impairment of the DNA damage repair, an important hallmark of GTXinduced carcinogenesis. Overall, our results indicate that microRNAs represent yet another relevant intracellular regulatory level in chemical carcinogenesis. 


\section{Introduction}

At present, cancer is the leading cause of death worldwide. The high prevalence of cancer, in especially the Western world, is caused by a combination of genetic and environmental factors (e.g. chemical agents $)^{1}$. Chemical carcinogenesis is a complex multistep process which is not well understood. Compounds may induce cancer by diverse mechanisms but are with respect to their mode of action (MOA) generally classified as genotoxic (GTX) or non-genotoxic (NGTX).

Whole genome-wide gene expression studies have been proven to be successful in gaining knowledge on the MOA of GTX and NGTX carcinogens ${ }^{2-10}$. These studies indicated that discriminative mRNA signature gene sets are indicative for their carcinogenic MOA. Thus by analyzing merely transcriptomics results it has been shown that compounds not only have a unique response however they also respond in a group-specific way. Earlier studies have established that DNA-reactive GTX carcinogens in general activate p53 tumor suppressor gene products in response to DNA damage, initiating characteristic processes including; DNA damage response, DNA repair processes, apoptosis and cell cycle arrest ${ }^{11}$. When DNA damage accumulates and its repair is not sufficient; mutations occur leading to the development of cancer. Non-DNA reactive NGTX carcinogens act via a range of mechanisms including increased proliferation, decreased apoptosis, energy depletion, production of reactive oxygen species and lipid peroxidation ${ }^{12}$. Furthermore they have been shown to act as tumor promoters by for example acting as peroxisome proliferators, endocrine-modifiers, receptormediators or immunosuppressants ${ }^{10,13}$.

As a consequence of the emphasis of 'omics-based explorative studies, in recent years, a systems biology approach has been adopted ${ }^{14-18}$ which focusses, besides on transcriptomics, on different molecular layers of the transcription regulation machinery. Within this context, non-coding RNA molecules are of interest because they act on a post-transcriptional level and regulate a large spectrum of essential processes by tuning the expression level of hundreds of mRNAs.

Aberrant microRNA levels have been shown to be associated with various types of cancer, such as hepatocellular carcinoma (e.g. microRNA-122, microRNA-21 and microRNA-199a-3p) 19,20 which raises the question if changes also occur in normal, healthy tissue following carcinogen exposure. Several studies have been performed, focusing on the role of microRNA changes in chemical carcinogenesis, evaluating multiple species (e.g. rat, human, mouse), tissues or cell types (e.g. lung, blood, colon) both in vitro as well as in vivo ${ }^{21}$.

Till date, mainly mice in vivo studies have been performed to examine the involvement of hepatic microRNAs in chemical carcinogenesis in general, in response to exposure to a single compound ${ }^{22-26}$. These studies resulted in the identification of specific hepatic microRNA expression profiles (e.g. microRNA-34a family 22 and microRNA-let-7C 25,26. MicroRNA expression was also analyzed in a small number of in vitro mouse hepatoma cell line studies 24,25, which are obviously not representative for the normally functioning liver. Against this background, we decided to study normal liver cells in vitro (e.g. primary hepatocytes) thereby focusing on investigating microRNA profiles following treatment to a spectrum of chemical carcinogens.

Within this study we hypothesize that microRNA response pathways exist for a group of xenobiotic agents (GTX and NGTX carcinogens). Furthermore, the role of the collective microRNA response as a function of specific chemical exposure will be further investigated. 
In this study a frequently used in vitro liver model, the primary mouse hepatocyte (PMH) model, was selected for evaluating relevant microRNA-based mechanisms induced by prototypical GTX and NGTX carcinogens. For this study three DNA damaging GTX carcinogens (aflatoxin B1 (AFB1), benzo(a)pyrene $(\mathrm{BaP})$ and cisplatin (CisPl)) as well as three NGTX carcinogens with various MOAs (2,3,7,8-tetrachloordibenzodioxine (TCDD) (arylhydrocarbon receptor agonist), cyclosporine A (CsA) (immunosuppressant) and $\mathrm{W}_{\mathrm{y}-}$ 14,643 (Wy) (peroxisome proliferator) were selected. PMH were treated with sub-cytotoxic doses and mRNA and microRNA levels were studied after two exposure periods (24 and 48h). This integration of $\mathrm{mRNA}$ and microRNA profiling using experimentally validated microRNA-mRNA interactions, may offer new prospects for understanding the generic and specific MOA of GTX and NGTX carcinogens. Consequently, the present study is one of the first mouse in vitro studies that sheds light on common microRNA response pathways affected by GTX and NGTX carcinogens in PMH and reveals a potential dual role for microRNAs in hepatic proliferation. Furthermore, it provides information on the role of microRNAs in the various compound-specific MOAs of GTX carcinogens in for example xenobiotic metabolism but also cell cycle arrest and DNA damage repair.

\section{Materials and methods}

\section{Chemicals}

All chemicals were obtained through Sigma-Aldrich, except for TCDD (Cerilliant). Genotoxicity of these compounds was based on information of both in vitro (Ames, mouse lymphoma assay, combination of in vitro chromosomal aberration and micronucleus test) as well as in vivo assays (micronucleus, chromosomal aberration, transgenic rodent assay) ${ }^{27-34}$. According to a report by the Food and Drug Administration ${ }^{35}$, a compound is considered GTX in vitro or in vivo when at least one in vitro or in vivo genotoxicity assay showed clear positive results. Carcinogenicity of these compounds was based on human carcinogen classification information from the International Agency for Research on Cancer ${ }^{36}$, National Toxicology Program ${ }^{37}$ or on other available literature on carcinogenicity assays ${ }^{38}$ (see Table 3.1).

\section{Cell culture and treatment}

Primary mouse hepatocytes (PMH) were isolated from adult male C57BL/6 mice using a previously published perfusion method, and cultured between a collagen-collagen sandwich formation in two six wells plates as described by Mathijs et al. ${ }^{39}$. After a recovery period of $40-$ 42h, the Dulbecco's Modified Eagle's (Gibco BRL, Breda, The Netherlands) culture medium was replaced by culture medium containing either one of the 6 compounds or a vehicle control $(0.5 \%$ of DMSO or PBS).

Cells were exposed to aflatoxin B1 (AFB1), benzo(a)pyrene (BaP), cisplatin (CisPl), 2,3,7,8tetrachloordibenzodioxine (TCDD), cyclosporine A (CsA) or Wy-14,643 (Wy) (as shown in Table 3.1) for 24 or $48 \mathrm{~h}$ before being harvested. A dose causing minimal cytotoxicity after $24 \mathrm{~h}$ of exposure thus resulting in $\pm 80 \%$ viability (as it is relevant for evaluating carcinogenic events to select a dose which does not induce severe cell death), was established by the MTT assay (Mosmann, 1983), (results not shown). Independent biological experiments (at least in triplicate) with hepatocytes from different mice were performed for each time point (except for Wy-14,643 which had four replicates). 


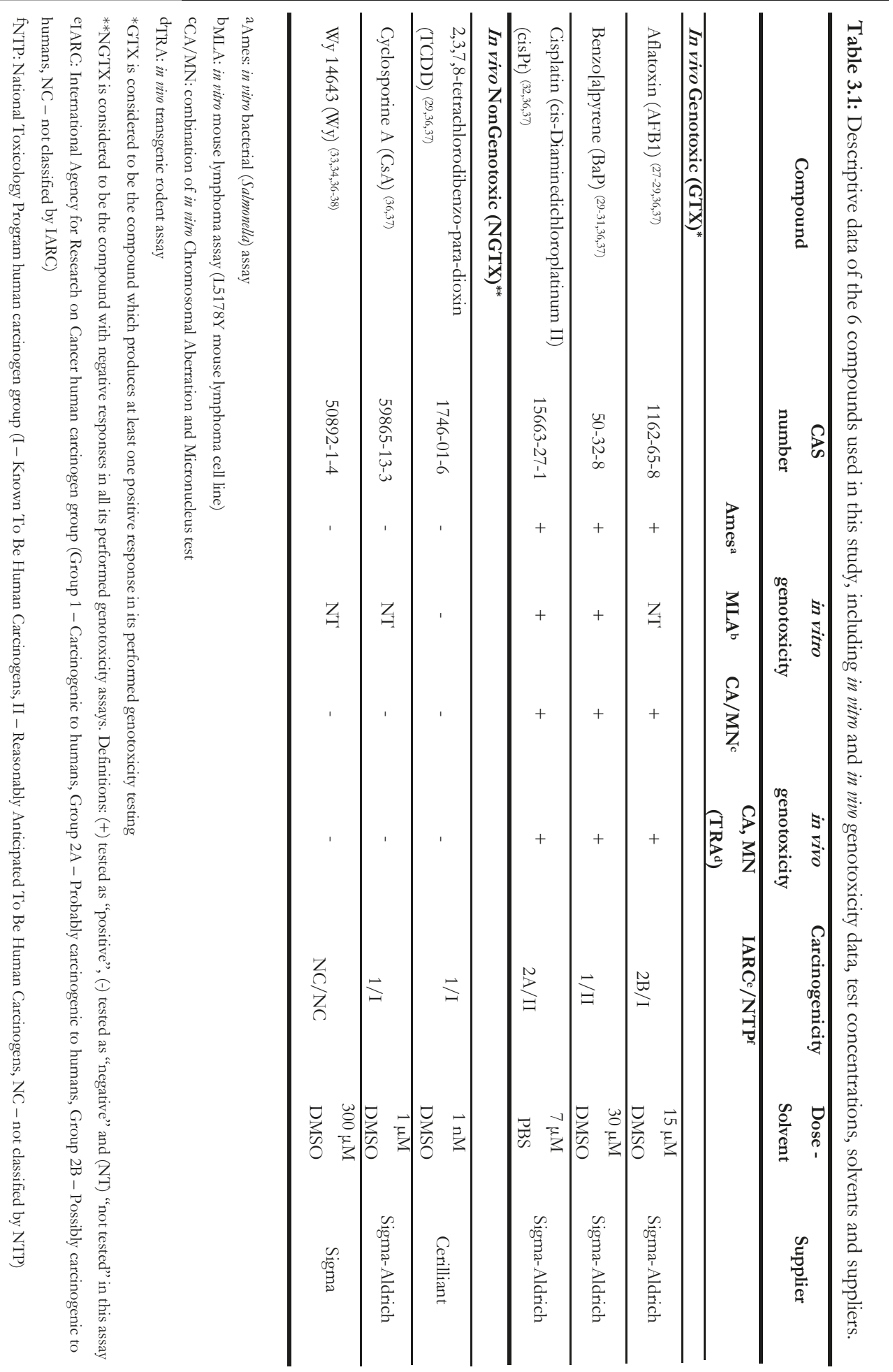




\section{RNA isolation and quality control}

At the end of treatment medium was removed and PMH were harvested in Qiazol (QIAGEN Benelux B.V., Venlo, The Netherlands). Total RNA was isolated using a miRNeasy Mini Kit (QIAGEN Benelux B.V., Venlo, The Netherlands) according to the manufacturer's protocol and followed by DNase I (Qiagen, Inc) treatment. Following purification, RNA concentrations were measured by means of a NanoDrop ${ }^{\circledR}$ ND-1000 spectrophotometer (Thermo Scientific, Wilmington, USA) at 260 and $280 \mathrm{~nm}$. RNA quality and integrity were assessed by using automated gel electrophoresis on an Agilent 2100 Bioanalyzer system (Agilent Technologies Netherlands B.V., Amstelveen, The Netherlands). Only RNA samples which showed clear 18S and $28 \mathrm{~S}$ peaks and with an RNA integrity number higher than 8, were used. Samples were stored at $-80^{\circ} \mathrm{C}$ until RNA hybridization.

\section{Gene expression analyses using Affymetrix arrays}

\section{Labelling and hybridization}

High-density oligonucleotide GeneChips from Affymetrix were used to measure gene expression levels (Mouse Genome 4302.0 array (45101 probes)). Targets for these arrays were prepared from $250 \mathrm{ng}$ of total RNA by means of the GeneChip 3' IVT Express Kit according to the Affymetrix protocol (Affymetrix UK Ltd, High Wycombe, UK). Amplified, biotinylated and fragmented targets were then hybridized on to the arrays. After hybridization, arrays were washed and stained using an Affymetrix fluidics station and scanned by use of an Affymetrix GeneArray scanner.

A total of 60 RNA samples was prepared and analyzed on GeneChip arrays.

Normalization quality controls, including scaling factors, average intensities, present calls, background intensities, noise, and raw $\mathrm{Q}$ values, appeared to be within acceptable limits for all chips. Hybridization controls BioB, BioC, BioD, and CreX were called present on all chips and yielded the expected increases in intensities.

The mRNA data discussed in this publication have been deposited in NCBI's Gene Expression Omnibus ${ }^{40}$ and are accessible through GEO Series accession number GSE57129.

\section{$\underline{\text { Re-annotation, normalization, and data filtering }}$}

All raw datasets from generated CEL files were first imported into R2.15.0 and run through an in-house quality control pipeline (available through ArrayAnalysis.org ${ }^{41}$ ) and then used for statistical analysis. $\mathrm{R}$ package is freely available for academic use from the Comprehensive $\mathrm{R}$ Archive Network (http://www.cran.r-project.org/). The probe sets were re-annotated by use of custom Cell Definition Files (version 17.0.0. http://brainarray.mbni.med.umich.edu/Brainarray/Database/CustomCDF/17.0.0/entrezg.as p). After re-annotation, data were normalized by Robust Multichip Average normalization and $\log 2$ transformed using the R package "affy". Low expression genes were filtered out by using a $\log [$ intensity cutoff $]>5$. This cutoff was based on the 1 st quartile of the distribution of average intensities of all reporters. Filtering out low expressed genes reduces the amount of noise being measured and thus excludes non-informative genes ${ }^{42,43}$. After the low expression genes were filtered out a list of 11,029 unique gene IDs remained for further analysis. Log2 ratios were then calculated based on average intensities (after filtering) of the treated and control samples. Gene sets were then selected using two approaches. 


\section{$\underline{\text { Selection of discriminative gene sets }}$}

In order to identify discriminative gene sets a selection of genes was made using the triplicate $\log 2$ ratios (treatment-control). To eliminate compound-specific effects contributing to gene expression changes, a leave-1-compound-out t-test $(\mathrm{p}<0.01)$ was performed as previously described and used by others ${ }^{44,45}$. For this purpose, the set of compounds was divided into GTX and NGTX carcinogens. For each t-test, the three replicates of one of the six chemicals were removed. The significant genes that were present in the intersection of all lists resulting from the six t-tests were selected as discriminative genes. To reduce the list of discriminative genes, the pre-selected set of features was loaded into PAM (Prediction Analysis for Microarrays, Stanford University Software, version 2.21), a frequently used "nearest shrunken centroid" class prediction program ${ }^{44,45}$. However in this exercise we were merely interested in the discriminative gene sets in a biological context instead of the predictive power of these genes. Therefore, the data were trained, cross-validated and the criterion for further reduction was chosen based on the best threshold matching our requirements of a low misclassification error and a strong reduction in the number of features. For GTX versus NGTX carcinogens a threshold for $24 \mathrm{~h}$ (1.67) resp. 48h (0.57) was chosen which resulted in 545 resp. 895 discriminative genes. Two-dimensional hierarchical clusters (Pearson correlation, Pairwise complete-linkage) were generated for visualization of the triplicate $\log 2$ ratios of the discriminative gene sets of $24 \mathrm{~h}$ and $48 \mathrm{~h}$ using Gene Pattern (version 3.9.1, http://genepattern.broadinstitute.org $\left.{ }^{46}\right)$. These discriminative gene sets were then used as input lists for overrepresentation analysis in ConsensusPathDB.

\section{Selection of compound-specific Differentially Expressed mRNAs (DE-mRNAs)}

Log2 ratios were used for selecting compound-specific DE-mRNAs based on a paired Student's t-test ( $\mathrm{p}$-value $<0.05$, unadjusted ) within $\mathrm{R}$ and an absolute fold changes of 1.5 for every compound separately ${ }^{47}$. For comparisons of the different compounds, lists of DEmRNAs were used for the generation of Venn diagrams.

Biological function of the discriminative mRNAs and DE-mRNAs

For biological interpretation of the discriminative gene sets, pathway analysis was performed by using over-representation analysis within ConsensusPathDB (http://cpdb.molgen.mpg.de/MCPDB). ConsensusPathDB-mouse integrates interaction networks in Mus musculus including binary and complex protein-protein, genetic, metabolic, signaling, gene regulatory and drug-target interactions, as well as biochemical pathways. Data originate from currently 16 public resources for interactions and interactions that have been curated from the literature. The interaction data were integrated in a complementary manner (avoiding redundancies), resulting in a seamless interaction network containing different types of interactions ${ }^{48}$. The criteria for significantly enriched pathways were; involvement of more than 5 genes from the used input list of gene sets and a p-value cutoff of 0.01 . A background list was used containing all 11029 genes that could be detected on the mRNA array.

The multiple testing corrected p-value was calculated according to the hypergeometric test based on the number of genes present in both the background list and the input list of gene sets. Following pathway analysis, molecular concepts were visualized. In these molecular concept maps, each node represents a separate concept whose member list size and p-value were encoded as node size and node color, respectively. This visual representation helps to quickly identify related biological processes that together show a changed activity, e.g. because they have the same key genes.

Pathway visualization was then performed within PathVisio ${ }^{49}$ using the MAPPBuilder plugin. 


\section{Determining MicroRNA expression using Exiqon arrays.}

\section{Labelling and hybridization}

The miRCURYTM locked-nuclei acid array (LNA), $5^{\text {th }}$ and $7^{\text {th }}$ generation (Exiqon, Denmark) contains probes that detect mature forms of all microRNAs present in miRBase 20.0 (http://www.mirbase.org/) ${ }^{50}$. The Exiqon platform has been validated and has been shown to reliably detect microRNA expression. The $5^{\text {th }}$ and $7^{\text {th }}$ generation microarray contains 9,360 and 14,708 reporters, which represent several control probes and sequences of mature human/mouse/rat specific microRNA (4 technical replicates). In our analysis, 661 unique mouse reporters (measurable on both generation arrays) were used. cDNA was generated using $1 \mu \mathrm{g}$ of total RNA per sample. RNA was labeled only with Hy3 containing dye (single color) using the mercury LNA ${ }^{\mathrm{TM}}$ microRNA Hy3 Power labeling kit (Exiqon, Denmark) according to the manufacturer's protocol in a total volume of $12.5 \mu$ l. To adjust the volume to $200 \mu \mathrm{l}$, nuclease free water was added to the labeled samples. Thereafter $200 \mu$ l hybridization buffer provided in miRCURY LNA ${ }^{\mathrm{TM}}$ microRNA Array, 5th generation kit (Exiqon, Denmark) was added. In a total volume of $400 \mu \mathrm{l}$ the sample was denatured for 2 minutes at $95^{\circ} \mathrm{C}$, incubated on ice for at least 15 minutes and then spun down. Manual hybridization was performed using an Agilent hybridization SureHyb chamber kit and gasket slide kit at $56{ }^{\circ} \mathrm{C}$ for $16 \mathrm{~h}$ in a hybridization oven with rotation. Following manual hybridization the arrays were automatically washed in a Tecan HS4800 Pro Hybridization station according to Exiqon instructions. For each biological experiment, one hybridization per time point was conducted and one sample per array. After washing, the arrays were scanned using the GenePix 4000A scanner (Axon Instruments, Foster City, CA).

The microRNA data discussed in this publication have been deposited in NCBI's Gene Expression Omnibus ${ }^{40}$ and are accessible through GEO Series accession number GSE57082.

\section{Probe annotation, normalization and data filtering}

To quantify the signals, the images were processed in to GPR ( $\left.5^{\text {th }}\right)$ and TXT ( $\left.7^{\text {th }}\right)$ files through the GenePix Pro Sofware Suite version 3.0 by using the GAL file for probe annotation, as obtained from Exiqon. The GAL file was based on miRBase version 20.0. These GPR or TXT files were then imported in to R 2.11.0, for quality control and statistical analysis. First, the quality of all arrays was inspected using arrayQC, an in-house quality control pipeline that generates virtual images, boxplots, correlation plots, clustering images, MvA and PCA plots. Spike-ins were used for all arrays as an extra quality check. Within this dataset, no arrays were deviated technically. During data analysis the spot intensities were background-corrected and then filtered for low signals (i.e. intensity $<10$ ). Only the mouse microRNA reporter intensities (661 unique reporters x 4 technical replicates) were used for further analyses. If for a reporter less than 3 of the 4 technical replicates were left, then the reporter intensity on that specific reporter was set to NA. The data were $\log 2$ transformed, followed by a quantile normalization and a summarization step. In the summarization step, the median intensity from the remaining technical replicates per condition was calculated. Finally, for each reporter a cutoff filtering was applied using the median of the three biological replicates. Only reporters that had a median intensity $\geq 50$ for at least one condition of the 9 compounds tested, or their respective controls, passed this filtering per time point. After filtering, log2 ratios were calculated between average intensities of the treatment of its respective control. MicroRNAs were selected using two approaches. 


\section{Selection of discriminative microRNAs}

Triplicate $\log 2$ ratios were used to identify discriminative microRNAs. A similar leave-1compound-out t-test approach was followed to select discriminative microRNAs as was used to identify discriminative gene sets (see paragraph 2.4.3.). To further reduce the set of preselected microRNAs an additional step was followed within PAM. The set of pre-selected microRNAs was trained, cross-validated and based on an appropriate threshold (low misclassification error with a small number of genes) further reduced. At $24 \mathrm{~h}$ no discriminative microRNAs could be selected. At $48 \mathrm{~h}$ three microRNAs were identified as being discriminative microRNAs in GTX versus NGTX carcinogens. One two-dimensional hierarchical cluster (Pearson correlation, Pairwise complete-linkage) was generated for visualization of the triplicate $\log 2$ ratios of the discriminative microRNAs at $48 \mathrm{~h}$ using Gene Pattern (version 3.9.1, http://genepattern.broadinstitute.org ${ }^{46}$ ).

Selection of compound-specific differentially expressed microRNAs (DE-miRs)

Log2 ratios were used for selecting compound-specific DE-mRNAs based on a paired Student's t-test ( $\mathrm{p}$-value $<0.05$, unadjusted ) within $\mathrm{R}$ and an absolute fold changes of 1.5 for every compound separately ${ }^{47}$. For comparisons of the different compounds, lists of DE-miRs were used for the creation of Venn diagrams.

\section{Obtaining gene target information of the discriminative microRNAs and DE-miRs}

As means to acquire knowledge on the biological function of the discriminative microRNAs, target information was retrieved using a database with experimentally validated microRNAmRNA interactions (miRTarBase). MiRTarBase (http://mirtarbase.mbc.nctu.edu.tw/) has accumulated more than fifty thousand miRNA-target interactions (MTIs), which are collected by manually surveying pertinent literature after data mining of the text systematically to filter research articles related to functional studies of miRNAs. Generally, the collected MTIs are validated experimentally by reporter assays, western blot, microarray and next-generation sequencing experiments. While containing the largest amount of validated MTIs, the miRTarBase provides the most updated collection by comparing with other similar, previously developed databases ${ }^{51}$. Within this study we mainly focused on anti-correlating interactions (microRNA up/downregulated, mRNA down/upregulated) since microRNAs are expected to repress or stimulate gene expression.

\section{$\underline{\text { Results }}$}

\section{The role of microRNAs in discriminative processes between GTX and NGTX carcinogens}

\section{Identifying discriminative gene sets}

In order to determine discriminative processes between GTX and NGTX carcinogens, differences in gene expression changes were studied induced by the two groups of chemicals used within this study. After $24 \mathrm{~h}$ of exposure, this resulted in a list of 545 genes that showed discriminative expression patterns between GTX and NGTX carcinogens. A similar list could be obtained after a $48 \mathrm{~h}$ incubation period at which time point 895 genes showed discriminative expression patterns. The expression patterns of the discriminative genes at $24 \mathrm{~h}$ and $48 \mathrm{~h}$ are visualized by means of two-dimensional hierarchical clusters in Figure 3.1 and Figure 3.2 respectively. The hierarchical clusters clearly show a separation of the GTX from the NGTX compounds and the discriminative genes also display a distinct expression pattern between the two groups. 


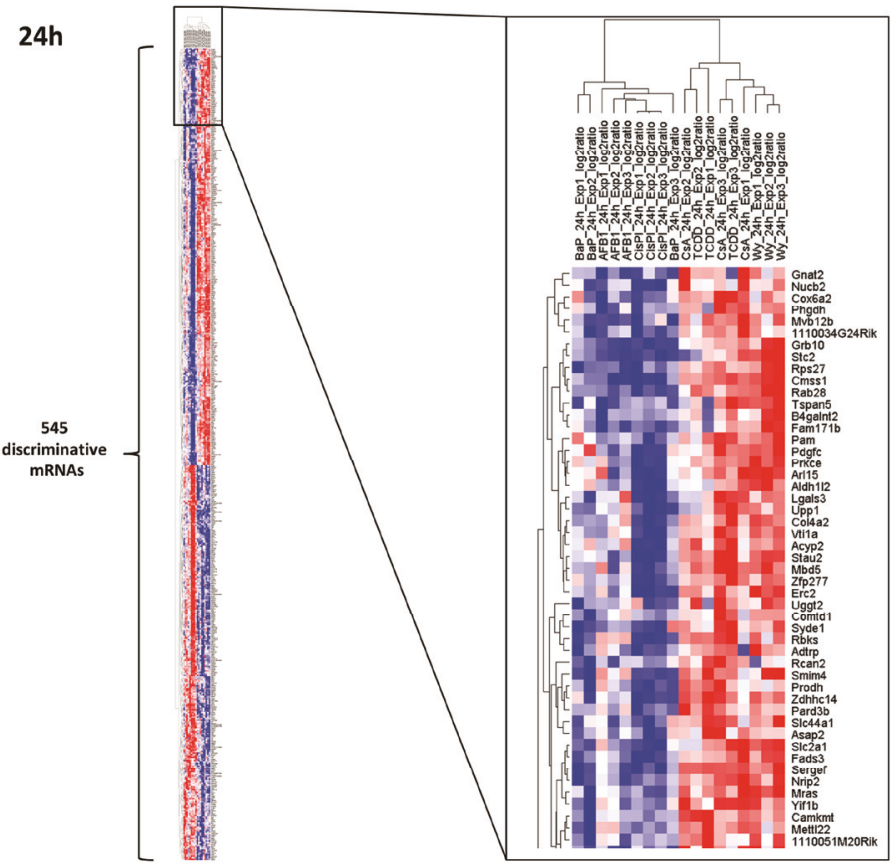

Figure 3.1: Two-dimensional hierarchical cluster (Pearson correlation, Pairwise complete-linkage created using Gene Pattern (version 3.9.1, http://genepattern.broadinstitute.org (46)) based on the triplicate log2 ratios (between -3 (blue) and 3 (red)) of the 545 discriminative gene sets found after $24 \mathrm{~h}$ of exposure.

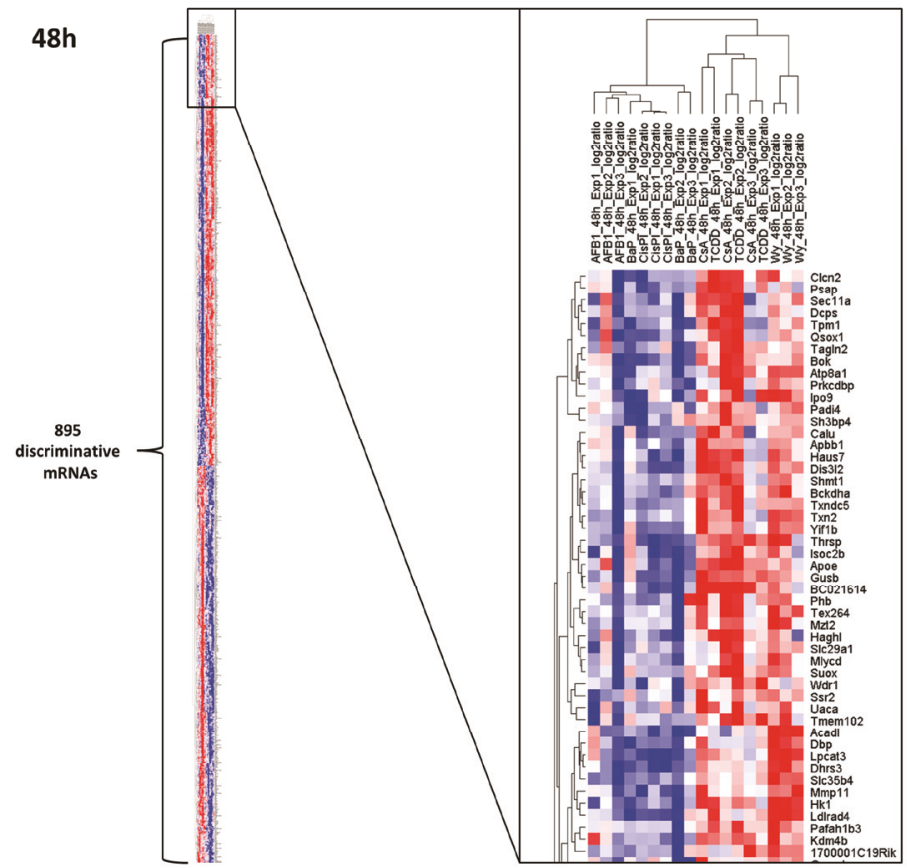

Figure 3.2: Two-dimensional hierarchical cluster (Pearson correlation, Pairwise complete-linkage created using Gene Pattern (version 3.9.1, http://genepattern.broadinstitute.org (46)) based on the triplicate log2 ratios (between -3 (blue) and 3 (red)) of the 895 discriminative gene sets found after $48 \mathrm{~h}$ of exposure. 
Determining discriminative processes using pathway analysis (ConsensusPathDB)

For pathway analysis, the obtained gene lists (545 and 895 genes) were fed into ConsensusPathDB. The over-representation analysis revealed that after $24 \mathrm{~h}$ of incubation, 264 discriminative genes (48.4\%) from the input list (545 genes) were present in at least one pathway. From the obtained pathways, 6 appeared to be significantly enriched ( $\mathrm{p}$-value $<0.01$, $>5$ genes involved) at $24 \mathrm{~h}$ of exposure (Supplementary Figure 3.1). Pathways were related to amino acid metabolism, energy metabolism, p53 signaling and signal transduction cascades. Gene expressions affected after GTX carcinogen treatment that were involved in metabolismrelated pathways were generally downregulated and genes involved in signal transduction cascades and p53 signaling were upregulated. In hepatocytes treated with NGTX carcinogens an opposite trend was observed implying an upregulation of metabolism-related genes and a downregulation of signal transduction cascades and p53 signaling genes. Details on the discriminative pathways at $24 \mathrm{~h}$ and their significance values can be found in part A of Supplementary Table 3.1.

A similar analysis after $48 \mathrm{~h}$ of incubation indicated that 456 genes $(50.9 \%)$ from the input list (895 genes) were present in at least one pathway. Twenty seven pathways were significantly enriched for the set of genes found discriminatively expressed at this time point. An expansion of metabolism-related processes could be identified indicated by enrichment of pathways involved in amino acid metabolism, lipid metabolism, cholesterol metabolism, energy metabolism and p53 signaling (Supplementary Figure 3.2). For the GTX carcinogens, downregulated genes were mainly involved in the metabolism-related pathways and upregulated genes for p53 signaling. For NGTX carcinogens, metabolism-related pathways involve upregulated genes and p53 signaling downregulated genes. Details on the discriminative pathways at $48 \mathrm{~h}$ and their significance values can be found in part B of Supplementary Table 3.1.

\section{Identifying potential discriminative microRNAs}

After 24 h of exposure, no microRNAs were found that showed distinctly different expression patterns between GTX and NGTX carcinogens. Three discriminative microRNAs could be identified after $48 \mathrm{~h}$ of exposure. The expression patterns of the discriminative microRNAs at $48 \mathrm{~h}$ are visualized by means of a two-dimensional hierarchical cluster in Figure 3.3.

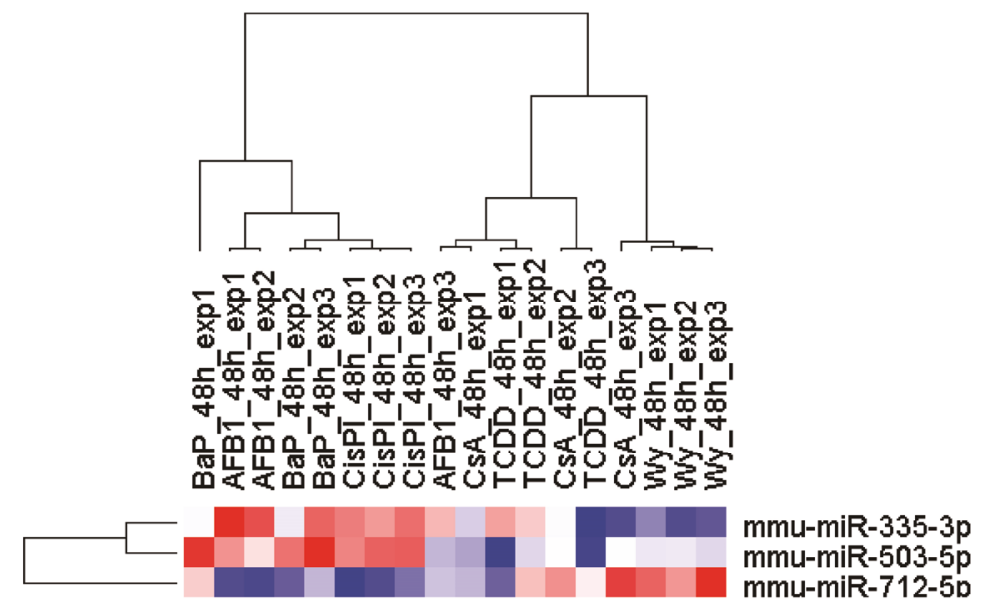

Figure 3.3: Two-dimensional hierarchical cluster (Pearson correlation, Pairwise complete-linkage created using Gene Pattern (version 3.9.1, http://genepattern.broadinstitute.org (46)) based on the triplicate $\log 2$ ratios (between -3 (blue) and 3 (red)) of the 3 discriminative microRNAs found after $48 \mathrm{~h}$ of exposure. 
The hierarchical cluster clearly shows a separation of the GTX from the NGTX compounds and the discriminative microRNAs also display a distinct expression pattern between the two groups.

Obtaining microRNA-mRNA interactions (miRTarBase) and exploring their role in discriminative processes

Information on microRNA-mRNA interactions was obtained using miRTarBase. The miRTarBase database contains 9195 experimentally validated microRNA - mRNA interactions for mouse. The 9195 microRNA - mRNA interactions imply 4346 genes and 180 microRNAs. The overlap between the microRNAs present in miRTarBase and the ones measurable on the Exiqon array (667 microRNAs measurable on array) was 146 microRNAs. So with respect to 521 microRNAs that in principle can be measured on the Exiqon array, no target information could be obtained. Therefore, from the 3 discriminative microRNAs that we obtained after $48 \mathrm{~h}$ of exposure, we were able to verify target information for one microRNA (mmu-miR-503$5 \mathrm{p})$. The overlap between the target genes present in miRTarBase and the ones measured on the Affymetrix array (11029 genes measurable on array) was 3266 genes. We were able connect one microRNA, found after $48 \mathrm{~h}$ of exposure, with 14 measurable targets. One gene target (Ccnd2) identified after $48 \mathrm{~h}$ of exposure, was present in the list of discriminative genes and showed to be involved in the discriminative process of "p53 signaling pathway". Ccnd2 demonstrated an anti-correlating interaction with mmu-miR-503-5p: after GTX exposure mmu-miR-503-5p appeared upregulated and cyclin D2 (Ccnd2 - 12444) downregulated while in NGTX exposed cells, mmu-miR-503-5p was downregulated and cyclin D2 upregulated. The mmu-miR-503-5p - Ccnd2 interaction was visualized within the "p53 signaling pathway" together with the other involved discriminative genes in Figure 3.4.

Besides the role of anti-correlating microRNAs in pathways distinguishing between molecular responses to GTX versus NGTX carcinogens, they appear also capable of regulating more compound-specific responses potentially related with their respective MOA. We noticed these compound-specific microRNA expression modifications by comparing individual microRNA expression levels between compounds. Results presented in Figure 3.6, depict the common and unique DE-miRs per compound after 24 (A and B) and 48h (C and D) of incubation respectively. The majority of the significant DE-miRs is, specifically at $48 \mathrm{~h}$, unique. Therefore, we proceeded with the following analysis focused on identifying compound-specific microRNA profiles. 


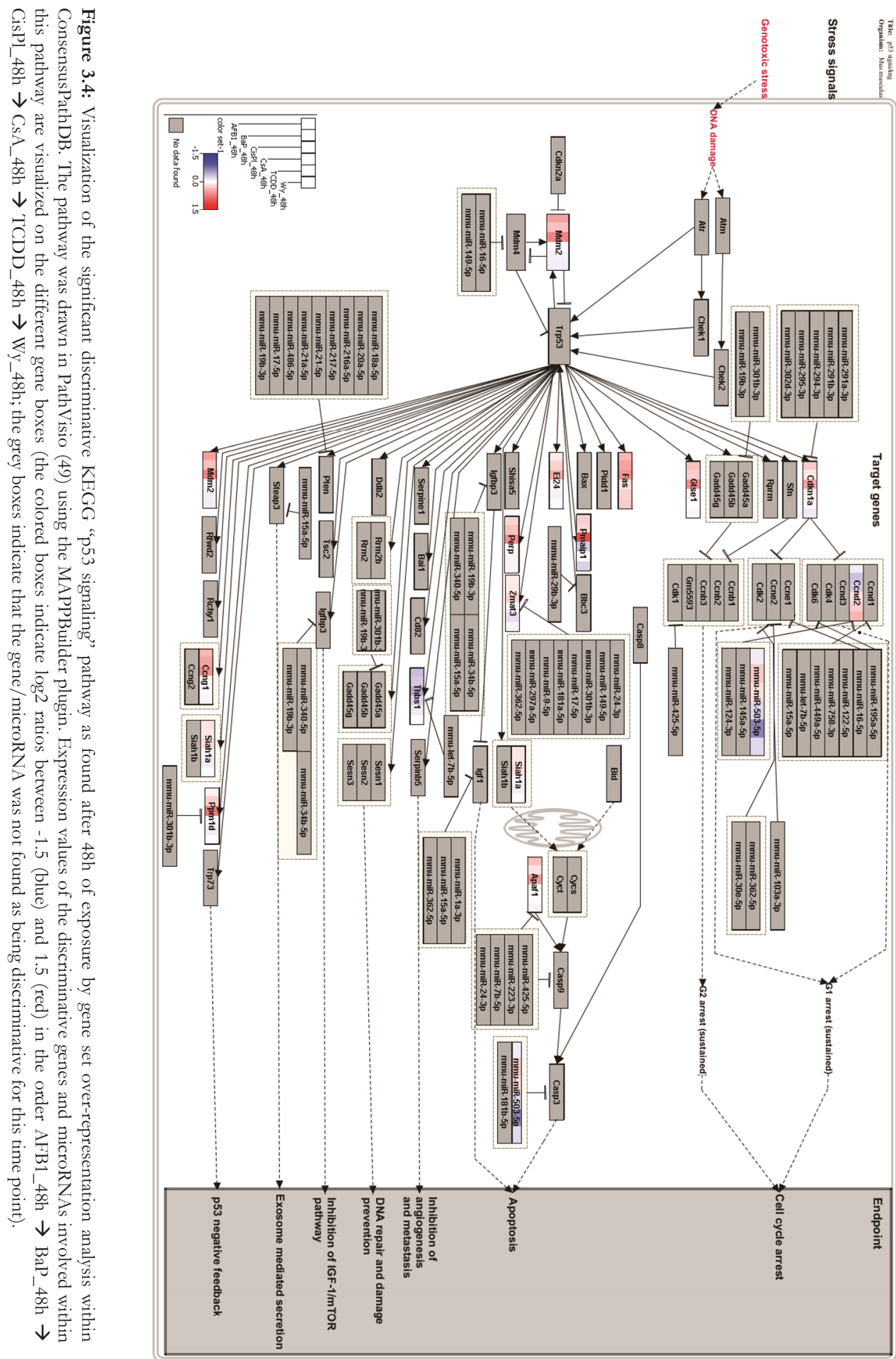




\section{The role of compound-specific microRNAs in the mode of action}

\section{Determining the compound-specific MOA using DE-mRNAs}

In order to gather information on the MOA of the individual compounds, over-representation analysis was performed using the DE-mRNAs. In Figure 3.5 the overlap and unique DEmRNAs after exposure for $24 \mathrm{~h}(\mathrm{~A}$ and $\mathrm{B})$ and $48 \mathrm{~h}$ (C and D) were shown respectively via a Venn diagram. The NGTX compounds, CsA, TCDD and Wy, are compared with the union of DE-mRNAs of the GTX group. Subsequently, the GTX compounds, AFB1, BaP and CisPl, are compared with the union of DE-mRNAs of the NGTX group. From this Venn diagram we can conclude that mRNA expression levels were compound-specific but also groupspecific. By analyzing the lists of DE-mRNAs, pathways could be obtained that could be linked with the MOA of these individual compounds as can be seen per compound in part A and C of Supplementary Table 3.2 for $24 \mathrm{~h}$ and $48 \mathrm{~h}$, respectively. Processes related to biotransformation, apoptosis, p53 signaling and DNA damage response were mainly apparent after exposure to $\mathrm{AFB} 1, \mathrm{BaP}$ and $\mathrm{CisPl}$. However, $\mathrm{BaP}$ and $\mathrm{CisPl}$ also regulate processes related to energy metabolism. Following CsA exposure, many processes related to the electron transport chain, appeared affected. TCDD influenced pathways related to drug metabolism and biotransformation. Wy exposure results in the over-representation of pathways with various functions: for example; energy (e.g. PPAR signaling and B-oxidation of fatty acids) and drug metabolism, biotransformation and chemical carcinogenesis.


Figure 3.5: The venn diagrams depict the overlapping and unique DE-mRNAs (based on their direction of change) between the $(\mathrm{A}, \mathrm{C})$ GTX carcinogens and CsA, TCDD and $\mathrm{Wy}_{\mathrm{y}}$ and the $(\mathrm{B}, \mathrm{D})$ NGTX carcinogens and AFB1, BaP and CisPl following $24 \mathrm{~h}$ (A, B) and $48 \mathrm{~h}$ (C,D) of exposure (DEG=DE-mRNAs, AFB1=aflatoxin B1, $\mathrm{BaP}=$ benzo(a)pyrene, $\mathrm{CisPl}=$ cisplatin, $\mathrm{CsA}=$ cyclosporin $\mathrm{A}, \mathrm{TCDD}=2,3,7,8$-tetrachloordibenzodioxine, $\mathrm{W}_{\mathrm{y}}=\mathrm{W}_{\mathrm{y}}$ 14,643) 
Determining unique differentially expressed microRNAs (DE-miRs)

In Figure 3.6 the overlap and unique DE-miRs after exposure for $24 \mathrm{~h}(\mathrm{~A}$ and $\mathrm{B})$ and $48 \mathrm{~h}(\mathrm{C}$ and D) were shown respectively via a Venn diagram. From these Venn diagrams we can conclude that microRNA expression levels were compound-specific. After $24 \mathrm{~h}$ of incubation this was not as clear since after this exposure period an overlap was observed between AFB1 and CsA or TCDD. At 48h, the response was more unique as illustrated by $\mathrm{BaP}$ and CisPl. After $48 \mathrm{~h}$ of exposure, the microRNA expression changes, in general, were more compoundspecific than overlapping between different compounds.

A.
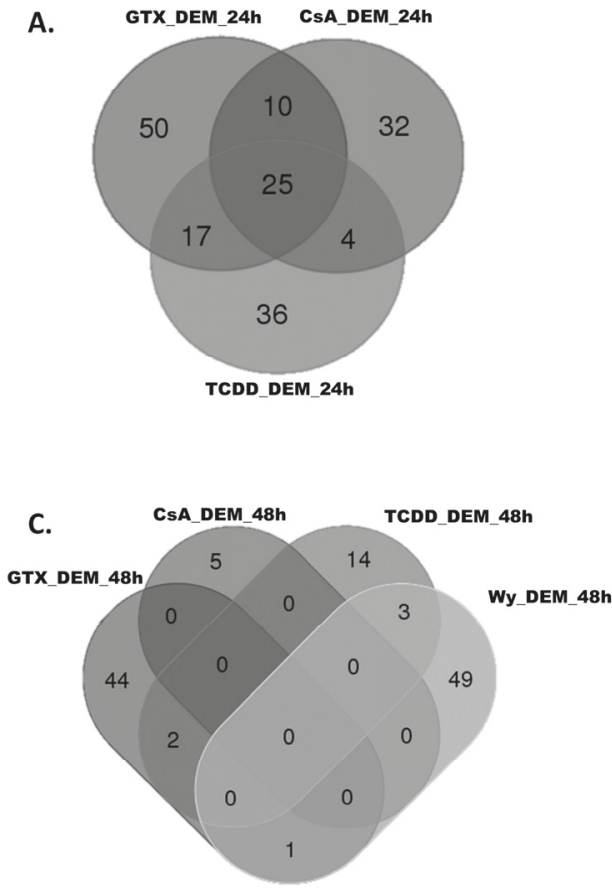
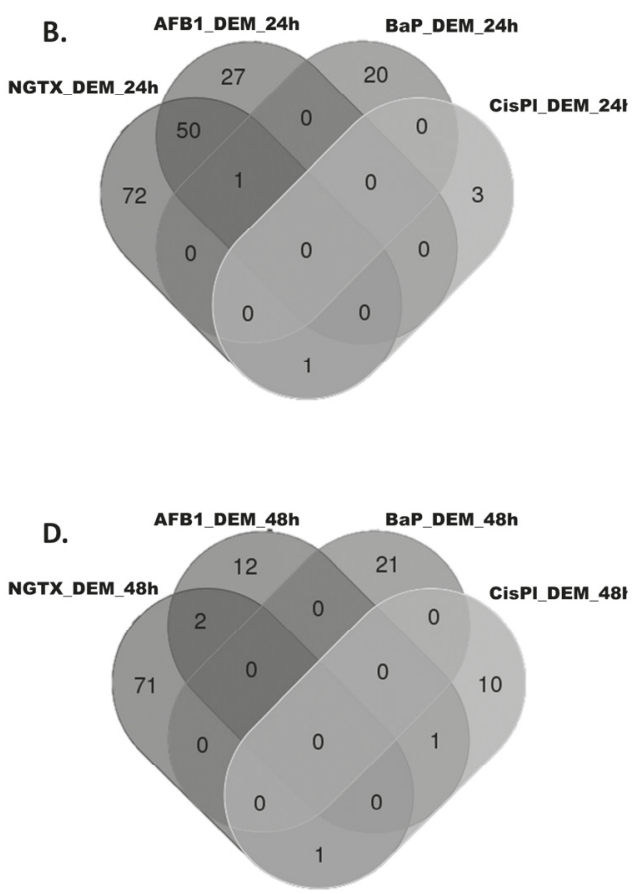

Figure 3.6: The venn diagrams depict the overlapping and unique DE-miRs (based on their direction of change) between the (A,C) GTX carcinogens and CsA, TCDD and Wy (only 48h) and the (B,D) NGTX carcinogens and $\mathrm{AFB} 1, \mathrm{BaP}$ and $\mathrm{CisPl}$ following $24 \mathrm{~h}$ (A, B) and 48h (C,D) of exposure (DEM=DE-miRs, AFB1=aflatoxin B1, $\mathrm{BaP}=$ benzo(a)pyrene, $\mathrm{Cis} \mathrm{Pl}=$ cisplatin, $\mathrm{CsA}=$ cyclosporin $\mathrm{A}, \mathrm{TCDD}=2,3,7,8$-tetrachloordibenzodioxine, $\mathrm{W}_{\mathrm{y}}=\mathrm{W}_{\mathrm{y}-}$ 14,643)

Obtaining microRNA-mRNA interactions (miRTarBase) and exploring their role in specific $\underline{\mathrm{MOA}}$

For AFB1 after $24 \mathrm{~h}$ of exposure information for one correlating microRNA-mRNA interaction could be retrieved.

Using miRTarBase 9 validated microRNA-mRNA were found after $48 \mathrm{~h}$ of AFB1 exposure. Two of these interactions actually showed an anti-correlating interaction. From these two anticorrelating interactions only one appears to be involved in affected pathways, namely mmumiR-301b-3p decreases the expression of 3'-phosphoadenosine 5'-phosphosulfate synthase 2 (Papss2 - 23972) which was involved in the MOA-related pathways; "Biological oxidations" 
and "Phase II conjugation". These are important in the bioactivation and detoxification of xenobiotics such as AFB1.

Following BaP exposure during 24h, 2 microRNA-mRNA interactions could be retrieved. One of these had an anti-correlating interaction which involved mmu-miR-29b-3p which reduces collagen, type IV, alpha 2 (Col4a2 - 12827) expression. This plays a role in the MOA-related pathways; "Focal adhesion", "Extracellular matrix organization" and "Integrin cell surface interactions".

After 48h of $\mathrm{BaP}$ exposure, 14 microRNA-mRNA interactions could be identified. Nine out of these were anti-correlating interactions. Only one anti-correlating interaction, mmu-miR-24-3p which represses filamin, alpha (Flna - 192176) expression, was involved in the MOA-related pathways; "Platelet degranulation" and "Response to elevated platelet cytosolic $\mathrm{Ca}^{2+}$.

For TCDD at $24 \mathrm{~h}$ four microRNA-mRNA interactions were established. This involved two correlating and two anti-correlating interactions. None of the anti-correlating interactions was found to be involved in one of the MOA-related pathways regulated by TCDD (24h).

After $24 \mathrm{~h}$ of CsA exposure 4 validated microRNA-mRNA interactions were found. All four have an anti-correlating interaction. However, none of them were involved in the obtained affected MOA-related pathways for this particular compound.

For Wy after $48 \mathrm{~h}$ of exposure three microRNA-mRNA interactions were found. Two of them showed an anti-correlating interaction. One of the anti-correlating interactions, mmu-miR-16$5 \mathrm{p}$ which decreases cyclin D1 (Ccnd1 - 12443) expression, was involved in the MOA-related pathways; "Spinal Cord Injury" and "HTLV-I infection".

For CisPl and $\mathrm{Wy}_{y}$ at $24 \mathrm{~h}$ and $\mathrm{CisPl}, \mathrm{TCDD}$ and $\mathrm{CsA}$ at $48 \mathrm{~h}$ no information on validated microRNA-mRNA interactions could be obtained through miRTarBase.

\section{$\underline{\text { Discussion }}$}

In this study we evaluated microRNA and mRNA expression profiles in PMH capable of distinguishing GTX from NGTX carcinogens. We hypothesized that microRNAs regulate such group-specific patterns of gene expression. We additionally focused on unique compound-specific microRNA expression patterns.

Discriminative gene sets, microRNAs (not for $24 \mathrm{~h}$ ) and processes were identified following $24 \mathrm{~h}$ and $48 \mathrm{~h}$ of exposure, distinguishing between molecular responses induced by GTX or NGTX carcinogens.

From the 3 discriminative microRNAs found following $48 \mathrm{~h}$ of exposure, mmu-miR-503-5p revealed to have an interaction with mRNA target gene cyclin D2 (Ccnd2 - 12444) which was involved in the discriminative processes of p53 signaling and metabolism-related pathways. Based on expression data we were able to observe a repression of Ccnd 2 by mmu-miR-503-5p following GTX carcinogen exposure while a stimulation was observed within the NGTX carcinogens group.

Ccnd2 is part of the growth factor independent cell cycle progression in hepatocytes in vitro. Growth factor independent cell cycle progression, through cyclin D proteins, mainly occurs when PMH are cultured on an extracellular matrix such as collagen type I layer ${ }^{52-54}$. Cell cycle progression in PMH especially takes place in case of liver regeneration or proliferation ${ }^{55}$.

Mmu-miR-503-5p belongs to the extended microRNA-16 family due to the similarity in seed regions 56 and thus probably share mRNA targets. Furthermore, mmu-miR-503-5p, an 
intragenic microRNA located on chromosomal location X: 53,051,984-53,056,054 ${ }^{50}$, is capable of inducing G1 arrest by targeting a set of cell cycle regulators ${ }^{57}$. Furthermore it has been observed that DNA damage induced by GTX compounds triggers p53 to interact with the Drosha/DGCR8 processing complex through an association with RNA helicase p68, which modulates the processing of pri-miRNAs to pre-miRNAs ${ }^{58,59}$. Thereby, p53 enhances the post-transcriptional maturation of several miRNAs with a growth-suppressive function, including members of the microRNA-16 family in response to DNA damage ${ }^{60}$. In addition, functional studies showed that microRNA-503 suppressed the proliferation of human HepG2 cells by the induction of G1 phase arrest through Rb-E2F signaling pathways, and thus can function as a tumor suppressor (like microRNA-16 does as well) ${ }^{61}$.

A potential mechanism (based on literature and experimental data) for mmu-miR-503-5p on Ccnd2 following GTX and NGTX carcinogen exposure was suggested in Figure 3.7. Some NGTX carcinogens (Figure 3.7) are known to activate peroxisome proliferator-activated receptor alpha (PPAR-alpha), aryl hydrocarbon receptor (AhR) or cyclophilin A which in turn activate both c-Myc 25,62-64 and NF-kB ${ }^{65-68}$. Activation of c-Myc may lead to an increase in energy- and oxidative phosphorylation-related pathways as was observed within this study 69,70 . Especially, elevated glucose levels may lead to an increase of Ccnd2 which promotes cell cycle progression ${ }^{69}$. Additionally, c-Myc ${ }^{71,72}$ as well as NF-kB ${ }^{73}$ repress $\mathrm{p} 53$ expression which leads to a decreased expression of mmu-miR-503-5p 58,60. Mmu-miR-503-5p expression is also inhibited by NF-kB ${ }^{74}$ ) which leads to an increased expression of Ccnd2 ${ }^{61,75,76}$ ultimately affecting cycle progression. While neither decreases of p53 nor increases of NF-kB gene expression have been observed within this study, an increase in c-Myc expression has actually been demonstrated. Cell cycle progression leads to hepatocyte proliferation which is an important step in both the promotion as well as the progression of cancer (including hepatocellular carcinoma) ${ }^{77,78}$. The expression of $\mathrm{Ccnd} 2$ appears upregulated by the current selection of NGTX carcinogens which thus indicates a role for mmu-miR-503-5p in stimulating liver proliferation.

In Figure 3.7 we also suggest a mechanism for mmu-miR-503-5p/Ccnd2-mediated genotoxic carcinogenesis. GTX carcinogens induce DNA damage (e.g. DNA adducts or double strand breaks (DSB)) thereby triggering p53 signaling. P53 activation may lead to an inhibition of energy- or oxidative phosphorylation-related pathways ${ }^{79}$ such as the inhibition of glycolysis and gluconeogenesis 80,81 . Activation of p53 may suppress the transcription of glucose transporters GLUT1 and GLUT4 (and via NFxB inhibits GLUT3) along with the insulin receptor thus inhibiting cellular glucose uptake ${ }^{80,82}$. An increase in p53 expression has not been observed within this study, however a decrease in c-Myc expression was observed. Following GTX carcinogen exposure downregulation of genes involved in pathways related to glycolysis and gluconeogenesis have also been observed in the current study. The decrease in glucose can also directly affect the expression level of Ccnd2 ${ }^{69,70}$. Additionally, p53 overexpression decreases the expression levels of c-Myc ${ }^{71,72}$ and increases the levels of mmu-miR503-5p 58,60. An increase of mmu-miR-503-5p and a decrease of c-Myc lead to a decreased expression level of Ccnd2 ${ }^{61,83}$ which may ultimately induce cell cycle arrest. Cell cycle arrest following GTX carcinogen exposure is necessary for the cell to allow repair of DNA damage induced by GTX compounds. In the long run, impaired DNA repair may lead to the accumulation of mutations and to carcinogenesis.

Where cell cycle arrest and hepatocyte proliferation have also been found by others to be characteristic for GTX vs. NGTX carcinogen exposure, both in in vitro studies $4,5,84$ and in in vivo experiments 2,6 studies (applying a toxicogenomics approach) we suggest a dual role for Mmu-miR-503-5p over-expression, either leading to tumor-suppression following GTX 
carcinogen exposure (by causing cell cycle arrest) or acting as an oncogene following NGTX carcinogen exposure (by inducing cell proliferation). These results therefore implicate a role for mmu-microRNA-503-5p and Ccnd2 in common mechanisms within these groups of GTX and NGTX carcinogens however do not shed light on their role in the specific toxic MOA of these compounds.

\section{Potential mechanism of mmu-miR-503-5p following exposure to GTX or NGTX carcinogens}

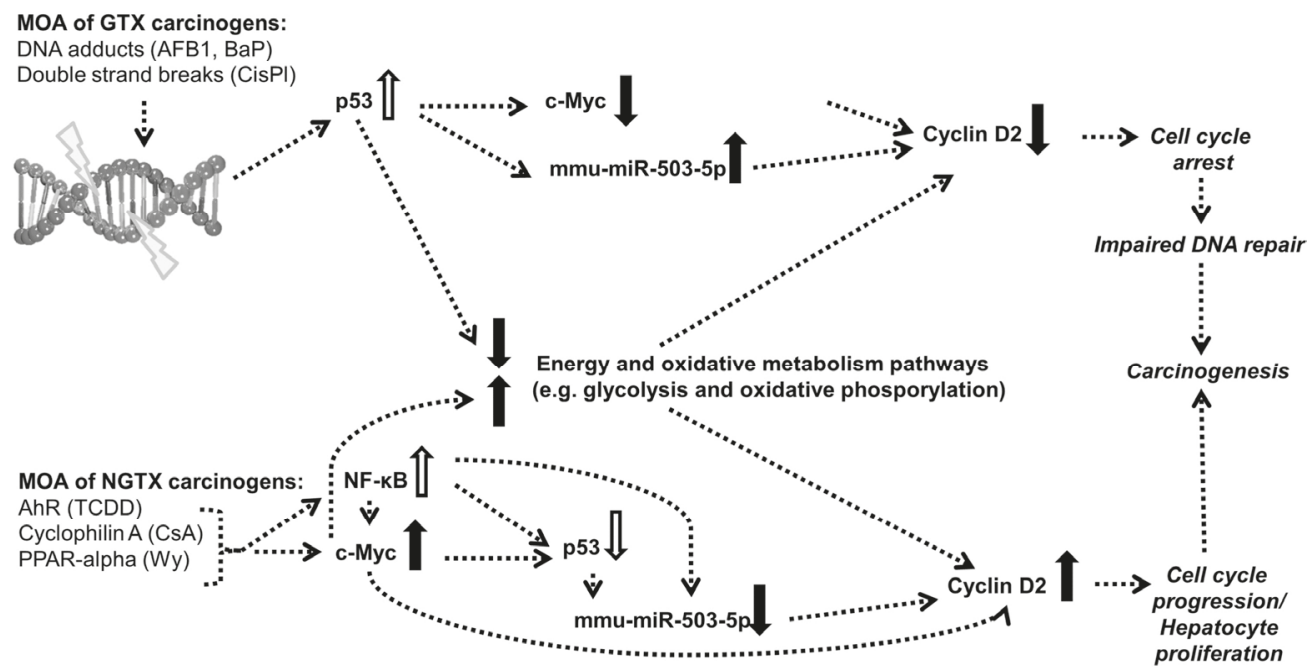

Figure 3.7: Visualization of the potential mechanism of mmu-miR-503-5p regulating cell cycle/proliferation and metabolism following exposure to GTX or NGTX carcinogens in primary mouse hepatocytes (GTX=genotoxic, NGTX $=$ non-genotoxic $\quad \mathrm{AFB} 1=$ aflatoxin $\quad \mathrm{B} 1, \quad \mathrm{BaP}=$ benzo(a)pyrene, $\quad \mathrm{CisPl}=$ cisplatin, $\mathrm{TCDD}=2,3,7,8$-tetrachloordibenzodioxine, $\mathrm{CsA}=$ cyclosporin $\mathrm{A}, \mathrm{W}_{\mathrm{y}}=\mathrm{W}_{\mathrm{y}}-14,643, \mathrm{AhR}=$ aryl hydrocarbon receptor, PPAR-alpha=peroxisome proliferator-activated receptor alpha, dotted line arrow $\rightarrow$ interactions based on literature; open arrows $\rightarrow$ expression values based on literature; solid arrows $\rightarrow$ expression values measured within this study (arrow up=up regulation, arrow down=down regulation))

Therefore in addition, we report interesting compound-specific responses apparent at the microRNA level as demonstrated by a limited overlap in differentially expressed microRNAs between the different compounds, mainly at $48 \mathrm{~h}$ of exposure. AFB1 exposure appeared to induce mmu-miR-301b-3p expression which may repress 3'-phosphoadenosine 5'phosphosulfate synthase 2 (Papss2 - 23972). AFB1 first needs to be converted by CYP3A4 in the liver to phase I metabolites which results in the formation of AFB1-8,9-epoxides. Papss2 is important in the phase II biotransformation (conjugation) of phase I metabolites, particularly by acting as a sulfate transferase, which results in AFB1-sulfate conjugate ${ }^{85}$. In the current experiment Papss 2 was repressed by mmu-miR-301b-3p which can be explained by the fact that conjugation by sulfate transferase is not preferred in mice. Mmu-miR-301b-3p plays, besides a role in xenobiotic metabolism, also an oncogenic role since its upregulation is observed within various type of cancer ${ }^{86}$ like hepatocellular carcinoma ${ }^{87}$. Overexpression of mmu-miR-301b-3p may enhance its function as onco-miR by promoting cancer proliferation, invasion, and tumor growth ${ }^{88}$.

After $24 \mathrm{~h}$ of $\mathrm{BaP}$ treatment, mmu-miR-29b-3p was shown to repress the expression of collagen, type IV, alpha 2 (Col4a2 - 12827). Type IV collagen is localized in the basement membrane and is involved in the maintenance of the structure of the hepatic extracellular matrix (ECM). Maintaining an optimal ECM is crucial for a cells homeostatic state. Abnormal 
expression of microRNA-29b is thus associated with tumorigenesis and cancer progression 89,90. microRNA-29 family members (miR-29a, miR-29b and miR-29c) are known to upregulate p53 protein levels and induce p53-mediated apoptosis through repression of $\mathrm{p} 85 \alpha$, a regulatory subunit of PI3 kinase (PI3K) ${ }^{91}$. Therefore aberrations in the expression of microRNA-29b may facilitate the adaptation of cancer cells to the crucial growth environment and, in turn, facilitate the development of hepatocellular carcinoma ${ }^{92}$. Furthermore, microRNA-29b has already been shown to be related with BaP-induced apoptosis and DNA damage repair within human HepG2 cells ${ }^{93}$.

After 48h of BaP exposure, mmu-miR-24-3p appears to repress filamin, alpha (Flna - 192176). Flna codes for a protein which cross-links the actin cytoskeleton ${ }^{94}$. Remodeling of the cytoskeleton plays a role in cell proliferation ${ }^{95}$. Flna is also required for efficient regulation of early stages of DNA repair processes. Furthermore overexpression of microRNA-24-3p has been shown to be associated with the promotion of tumor cell invasion and migration in response to hypoxia ${ }^{96}$.

After 48h of Wy treatment mmu-miR-16-5p was indicated to decrease the expression of cyclin D1 (Ccnd1 - 12443). Ccnd1 targets Rb protein in late G1 phase. This can ultimately may to cell cycle arrest ${ }^{77}$. The overexpression of microRNA-16 has shown to inhibit cell proliferation, arrest the cell cycle and induce apoptosis ${ }^{97}$. Another study also showed that microRNA-16 triggers G1 cell cycle arrest by repressing several involved genes of which Ccnd1 expression ${ }^{98}$. As mentioned before Wy, acting through PPAR-alpha, is known to induce cell cycle progression and hepatocyte proliferation ${ }^{99}$. Inhibition of Ccnd1 would, in theory, lead to cell cycle arrest. However, several studies have shown that loss or repression of Ccnd1 does not completely inhibit the proliferative response of mouse liver to mitogenic stimuli 100 . Furthermore, in cancer Ccnd2 can overrule the effect of Ccnd1 leading to an increase of proliferation ${ }^{101}$.

Having all of this in mind it might be important to stress the fact that a study published by Izzotti et al., 2011102 reported on the fact that microRNA alterations induced by environmental carcinogens, occurring in healthy organisms are predictive of the future appearance of cancer only when these miRNA alterations are irreversible. The change from reversibility to irreversibility of miRNA alteration mainly depends on the dose and the duration of the exposure. Therefore it is necessary for future research to extend the current exposure time in order to observe irreversible effects.

\section{$\underline{\text { Conclusions }}$}

In this study on carcinogen-induced microRNA regulations, one of the first in primary mouse hepatocytes, we have demonstrated the added value of investigating microRNA-mRNA interactions, as particularly highlighted by the discovery of the dual role of the mmu-miR-503$5 \mathrm{p}-\mathrm{Ccnd} 2$ interaction, suggesting a proliferative response after challenging by NGTX carcinogens, while demonstrating induction of cell cycle arrest, thus presumable facilitating DNA repair processes, after exposure to GTX carcinogens. In depth analysis of microRNAmediated chemical carcinogenesis awaits further clarifications of the biological role of microRNAs and in particular, the extensive validation of mRNA targets. Furthermore it needs to be further investigated if the changes on the microRNA level also are persistent and irreversible when cells are exposed for a longer period of time. 


\section{Supplementary data}

Supplementary Tables 3.1 and 3.2 and Figures 3.1 and 3.2 are available at Mutagenesis Online. 


\section{$\underline{\text { References }}$}

1. Doll, R. \& Peto, R. The causes of cancer: quantitative estimates of avoidable risks of cancer in the United States today. J Natl Cancer Inst 66, 1191-308 (1981).

2. Ellinger-Ziegelbauer, H., Stuart, B., Wahle, B., Bomann, W. \& Ahr, H.J. Comparison of the expression profiles induced by genotoxic and nongenotoxic carcinogens in rat liver. Mutat Res 575, 61-84 (2005).

3. Hochstenbach, K. et al. Transcriptomic fingerprints in human peripheral blood mononuclear cells indicative of genotoxic and non-genotoxic carcinogenic exposure. Mutat Res 746, 124-34 (2012).

4. van Delft, J.H. et al. Discrimination of genotoxic from non-genotoxic carcinogens by gene expression profiling. Carcinogenesis 25, 1265-76 (2004).

5. Mathijs, K. et al. Discrimination for genotoxic and nongenotoxic carcinogens by gene expression profiling in primary mouse hepatocytes improves with exposure time. Toxicol Sci 112, 374-84 (2009).

6. Lee, S.J. et al. Distinguishing between genotoxic and non-genotoxic hepatocarcinogens by gene expression profiling and bioinformatic pathway analysis. Sci Rep 3, 2783 (2013).

7. Iida, M. et al. Unique patterns of gene expression changes in liver after treatment of mice for 2 weeks with different known carcinogens and non-carcinogens. Carcinogenesis 26, 689-99 (2005).

8. Aubrecht, J. \& Caba, E. Gene expression profile analysis: an emerging approach to investigate mechanisms of genotoxicity. Pharmacogenomics 6, 419-28 (2005).

9. Fielden, M.R. et al. Interlaboratory evaluation of genomic signatures for predicting carcinogenicity in the rat. Toxicol Sci 103, 28-34 (2008).

10. Nie, A.Y. et al. Predictive toxicogenomics approaches reveal underlying molecular mechanisms of nongenotoxic carcinogenicity. Mol Carcinog 45, 914-33 (2006).

11. Ellinger-Ziegelbauer, H., Stuart, B., Wahle, B., Bomann, W. \& Ahr, H.J. Characteristic expression profiles induced by genotoxic carcinogens in rat liver. Toxicol Sci 77, 19-34 (2004).

12. Scott, D. et al. International Commission for Protection Against Environmental Mutagens and Carcinogens. Genotoxicity under extreme culture conditions. A report from ICPEMC Task Group 9. Mutat Res 257, 147-205 (1991).

13. Fielden, M.R. et al. Development and evaluation of a genomic signature for the prediction and mechanistic assessment of nongenotoxic hepatocarcinogens in the rat. Toxicol Sci 124, 54-74 (2011).

14. Heijne, W.H., Kienhuis, A.S., van Ommen, B., Stierum, R.H. \& Groten, J.P. Systems toxicology: applications of toxicogenomics, transcriptomics, proteomics and metabolomics in toxicology. Expert Rev Proteomics 2, 767-80 (2005).

15. Olden, K. Toxicogenomics--a new systems toxicology approach to understanding of gene-environment interactions. Ann N Y Acad Sci 1076, 703-6 (2006).

16. Plant, N. Can systems toxicology identify common biomarkers of non-genotoxic carcinogenesis? Toxicology 254, 164-9 (2008).

17. Ament, Z. et al. A metabolomics investigation of non-genotoxic carcinogenicity in the rat. J Proteome Res 12 , 5775-90 (2013).

18. Jennen, D. et al. Integrating transcriptomics and metabonomics to unravel modes-of-action of 2,3,7,8tetrachlorodibenzo-p-dioxin (TCDD) in HepG2 cells. BMC Syst Biol 5, 139 (2011).

19. Hou, J. et al. Identification of miRNomes in human liver and hepatocellular carcinoma reveals miR-199a/b$3 \mathrm{p}$ as therapeutic target for hepatocellular carcinoma. Cancer Cell 19, 232-43 (2011).

20. Pineau, P. et al. miR-221 overexpression contributes to liver tumorigenesis. Proc Natl Acad Sci U S A 107, 264-9 (2010).

21. Izzotti, A. \& Pulliero, A. The effects of environmental chemical carcinogens on the microRNA machinery. International Journal of Hygiene and Environmental Health 217, 601-627 (2014).

22. Li, Z. et al. Genomic analysis of microRNA time-course expression in liver of mice treated with genotoxic carcinogen N-ethyl-N-nitrosourea. BMC Genomics 11, 609 (2010).

23. Yauk, C.L., Jackson, K., Malowany, M. \& Williams, A. Lack of change in microRNA expression in adult mouse liver following treatment with benzo(a)pyrene despite robust mRNA transcriptional response. Mutat Res 722, 131-9 (2011).

24. Moffat, I.D. et al. microRNAs in adult rodent liver are refractory to dioxin treatment. Toxicol Sci 99, 470-87 (2007).

25. Shah, Y.M. et al. Peroxisome proliferator-activated receptor alpha regulates a microRNA-mediated signaling cascade responsible for hepatocellular proliferation. Mol Cell Biol 27, 4238-47 (2007).

26. Zhang, B. \& Pan, X. RDX induces aberrant expression of microRNAs in mouse brain and liver. Environ Health Perspect 117, 231-40 (2009).

27. Corvi, R. et al. ECVAM retrospective validation of in vitro micronucleus test (MNT). Mutagenesis 23, 271-83 (2008). 
28. Kirkland, D., Kasper, P., Muller, L., Corvi, R. \& Speit, G. Recommended lists of genotoxic and nongenotoxic chemicals for assessment of the performance of new or improved genotoxicity tests: a follow-up to an ECVAM workshop. Mutat Res 653, 99-108 (2008).

29. Lambert, I.B., Singer, T.M., Boucher, S.E. \& Douglas, G.R. Detailed review of transgenic rodent mutation assays. Mutat Res 590, 1-280 (2005).

30. Valentin-Severin, I., Le Hegarat, L., Lhuguenot, J.C., Le Bon, A.M. \& Chagnon, M.C. Use of HepG2 cell line for direct or indirect mutagens screening: comparative investigation between comet and micronucleus assays. Mutat Res 536, 79-90 (2003).

31. Muller-Tegethoff, K., Kasper, P. \& Muller, L. Evaluation studies on the in vitro rat hepatocyte micronucleus assay. Mutat Res 335, 293-307 (1995).

32. Khynriam, D. \& Prasad, S.B. Cisplatin-induced genotoxic effects and endogenous glutathione levels in mice bearing ascites Dalton's lymphoma. Mutat Res 526, 9-18 (2003).

33. U.S. National Library of Medicine. Toxnet Toxicological Data Network (CCRIS,HSDB, ChemIDplus and Genetox). http://toxnet.nlm.nih.gov/, (accessed on November 14, 2014) (2014).

34. Kirkland, D., Reeve, L., Gatehouse, D. \& Vanparys, P. A core in vitro genotoxicity battery comprising the Ames test plus the in vitro micronucleus test is sufficient to detect rodent carcinogens and in vivo genotoxins. Mutat Res 721, 27-73 (2011).

35. Food and Drug Administration (FDA). International Conference on Harmonisation; guidance on S2(R1) Genotoxicity Testing and Data Interpretation for Pharmaceuticals intended for Human Use; availability. Notice. Fed Regist 77, 33748-9 (2012).

36. International Agency for Research on Cancer (IARC). IARC Monographs on the Evaluation of Carcinogenic Risks to Humans. http://monographs.iarc.fr/ENG/Classification/ClassificationsGroupOrder.pdf, (accessed on November 14, 2014) (2014).

37. US Department of Health and Human Services. Public Health Service. National Toxicology Program (NTP). Report on Carcinogens. http://ntp.niehs.nih.gov/pubhealth/roc/roc13/index.html, (accessed on November 14, 2014) (2014).

38. Reddy, J.K., Rao, M.S., Azarnoff, D.L. \& Sell, S. Mitogenic and carcinogenic effects of a hypolipidemic peroxisome proliferator, [4-chloro-6-(2,3-xylidino)-2-pyrimidinylthio]acetic acid (Wy-14, 643), in rat and mouse liver. Cancer Res 39, 152-61 (1979).

39. Mathijs, K. et al. Gene expression profiling in primary mouse hepatocytes discriminates true from falsepositive genotoxic compounds. Mutagenesis 25, 561-8 (2010).

40. Edgar, R., Domrachev, M. \& Lash, A.E. Gene Expression Omnibus: NCBI gene expression and hybridization array data repository. Nucleic Acids Res 30, 207-10 (2002).

41. Eijssen, L.M. et al. User-friendly solutions for microarray quality control and pre-processing on ArrayAnalysis.org. Nucleic Acids Res 41, W71-6 (2013).

42. Chen, J., Hsueh, H.-M., Delongchamp, R., Lin, C.-J. \& Tsai, C.-A. Reproducibility of microarray data: a further analysis of microarray quality control (MAQC) data. BMC Bioinformatics 8, 412 (2007).

43. Bourgon, R., Gentleman, R. \& Huber, W. Independent filtering increases detection power for highthroughput experiments. Proceedings of the National Academy of Sciences 107, 9546-9551 (2010).

44. Magkoufopoulou, C. et al. A transcriptomics-based in vitro assay for predicting chemical genotoxicity in vivo. Carcinogenesis 33, 1421-9 (2012).

45. Van den Hof, W.F. et al. Classification of hepatotoxicants using HepG2 cells: A proof of principle study. Chem Res Toxicol 27, 433-42 (2014).

46. Reich, M. et al. GenePattern 2.0. Nat Genet 38, 500-1 (2006).

47. Guo, L. et al. Rat toxicogenomic study reveals analytical consistency across microarray platforms. Nat Biotechnol 24, 1162 - 1169 (2006).

48. Kamburov, A., Wierling, C., Lehrach, H. \& Herwig, R. ConsensusPathDB--a database for integrating human functional interaction networks. Nucleic Acids Res 37, D623-8 (2009).

49. Kutmon, M. et al. PathVisio 3: An Extendable Pathway Analysis Toolbox. PLoS Comput Biol 11, e1004085 (2015).

50. Griffiths-Jones, S., Saini, H.K., van Dongen, S. \& Enright, A.J. miRBase: tools for microRNA genomics. Nucleic Acids Research 36, D154-8 (2008).

51. Hsu, S.-D. et al. miRTarBase: a database curates experimentally validated microRNA-target interactions. Nucleic Acids Research 39, D163-D169 (2011).

52. Nelsen, C.J. et al. Amino acids regulate hepatocyte proliferation through modulation of cyclin D1 expression. J Biol Chem 278, 25853-8 (2003).

53. Loyer, P. et al. Growth factor dependence of progression through G1 and S phases of adult rat hepatocytes in vitro. Evidence of a mitogen restriction point in mid-late G1. J Biol Chem 271, 11484-92 (1996).

54. Loyer, P. et al. Progression through G1 and S phases of adult rat hepatocytes. Prog Cell Cycle Res 2, 37-47 (1996). 
55.

56.

57.

58.

59.

60.

61.

63.

64.

65.

66.

67.

68.

69.

70.

71.

72.

73.

74.

75.

76.

Awad, M.M. \& Gruppuso, P.A. Cell cycle control during liver development in the rat: evidence indicating a role for cyclin D1 posttranscriptional regulation. Cell Growth Differ 11, 325-34 (2000).

Caporali, A. \& Emanueli, C. MicroRNA-503 and the extended microRNA-16 family in angiogenesis. Trends Cardiovasc Med 21, 162-6 (2011).

Forrest, A.R. et al. Induction of microRNAs, mir-155, mir-222, mir-424 and mir-503, promotes monocytic differentiation through combinatorial regulation. Leukemia 24, 460-466 (2010).

Boominathan, L. The tumor suppressors p53, p63, and $\mathrm{p} 73$ are regulators of microRNA processing complex. PLoS ONE 5, e10615 (2010).

Pothof, J. et al. MicroRNA-mediated gene silencing modulates the UV-induced DNA-damage response. EMBO J 28, 2090-9 (2009).

Hu, H. \& Gatti, R.A. MicroRNAs: new players in the DNA damage response. J Mol Cell Biol 3, 151-8 (2011).

Xiao, F. et al. MicroRNA-503 inhibits the G1/S transition by downregulating cyclin D3 and E2F3 in hepatocellular carcinoma. Journal of Translational Medicine 11, 195 (2013).

Peters, J.M. et al. Role of peroxisome proliferator-activated receptor alpha in altered cell cycle regulation in mouse liver. Carcinogenesis 19, 1989-94 (1998).

Miller, R.T. et al. Effect on the expression of c-met, c-myc and PPAR-alpha in liver and liver tumors from rats chronically exposed to the hepatocarcinogenic peroxisome proliferator WY-14,643. Carcinogenesis 17, 1337-41 (1996).

Kim, D.W. et al. The RelA NF-kappaB subunit and the aryl hydrocarbon receptor (AhR) cooperate to transactivate the c-myc promoter in mammary cells. Oncogene 19, 5498-506 (2000).

Teoh, N.C. et al. Short-term therapy with peroxisome proliferation-activator receptor-alpha agonist $\mathrm{W}_{\mathrm{y}-}$ 14,643 protects murine fatty liver against ischemia-reperfusion injury. Hepatology 51, 996-1006 (2010).

Tian, Y., Ke, S., Denison, M.S., Rabson, A.B. \& Gallo, M.A. Ah receptor and NF-kappaB interactions, a potential mechanism for dioxin toxicity. J Biol Chem 274, 510-5 (1999).

Barouki, R., Coumoul, X. \& Fernandez-Salguero, P.M. The aryl hydrocarbon receptor, more than a xenobiotic-interacting protein. FEBS Lett 581, 3608-15 (2007).

Andres, D., Diez-Fernandez, C., Zaragoza, A., Alvarez, A. \& Cascales, M. Induction of cell proliferation by cyclosporine A in primary cultures of rat hepatocytes. Biochem Pharmacol 61, 427-35 (2001).

Salpeter, S.J. et al. Glucose regulates cyclin D2 expression in quiescent and replicating pancreatic beta-cells through glycolysis and calcium channels. Endocrinology 152, 2589-98 (2011).

Fajas, L. Re-thinking cell cycle regulators: the cross-talk with metabolism. Front Oncol 3, 4 (2013).

Ceballos, E. et al. c-Myc antagonizes the effect of p53 on apoptosis and p21WAF1 transactivation in K562 leukemia cells. Oncogene 19, 2194-204 (2000).

Ho, J.S., Ma, W., Mao, D.Y. \& Benchimol, S. p53-Dependent transcriptional repression of c-myc is required for G1 cell cycle arrest. Mol Cell Biol 25, 7423-31 (2005).

Webster, G.A. \& Perkins, N.D. Transcriptional cross talk between NF-kappaB and p53. Mol Cell Biol 19, 3485-95 (1999).

Zhou, R. et al. Histone deacetylases and NF-kB signaling coordinate expression of CX3CL1 in epithelial cells in response to microbial challenge by suppressing miR-424 and miR-503. PLoS ONE 8, e65153 (2013).

Jiang, Q., Feng, M. \& Mo, Y. Systematic validation of predicted microRNAs for cyclin D1. BMC Cancer 9 , 194 (2009).

Liao, D.J., Thakur, A., Wu, J., Biliran, H. \& Sarkar, F.H. Perspectives on c-Myc, Cyclin D1, and their interaction in cancer formation, progression, and response to chemotherapy. Crit Rev Oncog 13, 93-158 (2007).

Mullany, L.K. et al. Distinct proliferative and transcriptional effects of the D-type cyclins in vivo. Cell Cycle 7, 2215-24 (2008).

Farber, E. Hepatocyte proliferation in stepwise development of experimental liver cell cancer. Dig Dis $S_{c i}$ 36, 973-8 (1991).

Wang, S.J. \& Gu, W. To be, or not to be: functional dilemma of p53 metabolic regulation. Curr Opin Oncol 26, 78-85 (2014).

Maddocks, O.D. \& Vousden, K.H. Metabolic regulation by p53. J Mol Med (Berl) 89, 237-45 (2011).

Yeung, S.J., Pan, J. \& Lee, M.H. Roles of p53, Myc and HIF-1 in Regulating Glycolysis — the Seventh Hallmark of Cancer. Cellular and Molecular Life Sciences 65, 3981-3999 (2008).

Kruse, J.P. \& Gu, W. Modes of p53 regulation. Cell 137, 609-22 (2009).

Jiang, Q., Feng, M.G. \& Mo, Y.Y. Systematic validation of predicted microRNAs for cyclin D1. BMC Cancer 9, 194 (2009).

Jennen, D.G. et al. Comparison of HepG2 and HepaRG by whole-genome gene expression analysis for the purpose of chemical hazard identification. Toxicol Sci 115, 66-79 (2010).

5. Maziasz, T.J., Liu, J., Madhu, C. \& Klaassen, C.D. The differential effects of hepatotoxicants on the sulfation pathway in rats. Toxicol Appl Pharmacol 110, 365-73 (1991). 
86. Funamizu, N. et al. MicroRNA-301b promotes cell invasiveness through targeting TP63 in pancreatic carcinoma cells. Int J Oncol 44, 725-34 (2014).

87. Jiang, J. et al. Association of MicroRNA expression in hepatocellular carcinomas with hepatitis infection, cirrhosis, and patient survival. Clin Cancer Res 14, 419-27 (2008).

88. Shi, W. et al. MicroRNA-301 mediates proliferation and invasion in human breast cancer. Cancer Res 71, 2926-37 (2011).

89. Cortez, M.A. et al. miR-29b and miR-125a regulate podoplanin and suppress invasion in glioblastoma. Genes Chromosomes Cancer 49, 981-90 (2010).

90. Huang, X. et al. Targeted delivery of microRNA-29b by transferrin-conjugated anionic lipopolyplex nanoparticles: a novel therapeutic strategy in acute myeloid leukemia. Clin Cancer Res 19, 2355-67 (2013).

91. Feng, Z., Zhang, C., Wu, R. \& Hu, W. Tumor suppressor p53 meets microRNAs. J Mol Cell Biol 3, 44-50 (2011).

92. Xiong, Y. et al. Effects of microRNA-29 on apoptosis, tumorigenicity, and prognosis of hepatocellular carcinoma. Hepatology 51, 836-45 (2010).

93. Lizarraga, D. et al. Benzo[a]pyrene-induced changes in microRNA-mRNA networks. Chem Res Toxicol 25, 838-49 (2012).

94. Kim, H. \& McCulloch, C.A. Filamin A mediates interactions between cytoskeletal proteins that control cell adhesion. FEBS Lett 585, 18-22 (2011).

95. Provenzano, P.P. \& Keely, P.J. Mechanical signaling through the cytoskeleton regulates cell proliferation by coordinated focal adhesion and Rho GTPase signaling. J Cell Sci 124, 1195-205 (2011).

96. Du, W.W. et al. MicroRNA miR-24 enhances tumor invasion and metastasis by targeting PTPN9 and PTPRF to promote EGF signaling. J Cell Sci 126, 1440-53 (2013).

97. Guo, C.J., Pan, Q., Jiang, B., Chen, G.Y. \& Li, D.G. Effects of upregulated expression of microRNA-16 on biological properties of culture-activated hepatic stellate cells. Apoptosis 14, 1331-40 (2009).

98. Liu, Q. et al. miR-16 family induces cell cycle arrest by regulating multiple cell cycle genes. Nucleic Acids Res 36, 5391 - 5404 (2008).

99. Woods, C.G. et al. WY-14,643-Induced Cell Proliferation and Oxidative Stress in Mouse Liver are Independent of NADPH Oxidase. Toxicological Sciences 98, 366-374 (2007).

100. Ledda-Columbano, G.M. et al. Loss of cyclin D1 does not inhibit the proliferative response of mouse liver to mitogenic stimuli. Hepatology 36, 1098-105 (2002).

101. Ely, S. et al. Mutually exclusive cyclin-dependent kinase 4/cyclin D1 and cyclin-dependent kinase 6/cyclin D2 pairing inactivates retinoblastoma protein and promotes cell cycle dysregulation in multiple myeloma. Cancer Res 65, 11345-53 (2005).

102. Izzotti, A. et al. Dose-responsiveness and persistence of microRNA expression alterations induced by cigarette smoke in mouse lung. Mutat Res 717, 9-16 (2011). 
()

0

$\bigcirc$

00

$\mathrm{O}$

0

$\therefore 0$

Chapter 4

O

Improving the predictability of in vivo () genotoxicity and carcinogenicity by exploiting microRNA and mRNA profileś

()$\bigcirc$ generated in vitro from exposed primary

O

O mouse hepatocytes.

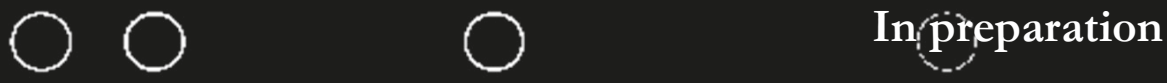

0
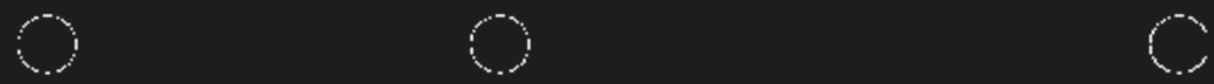

0

00

00
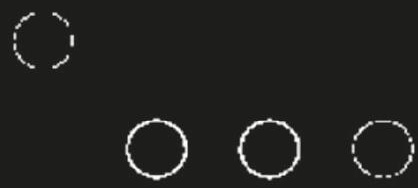

Rieswijk L.

Brauers K打

Coonen M:L.J.

van Breda S.G.J.

Jennen, D.G.J.

Kleinjans J.C.S. 


\section{$\underline{\text { Abstract }}$}

Till date, chemical cancer risk assessment relies on a well-defined battery of in vitro- systems. These, however, are characterized by a high false-positive rate, thus repeatedly failing to correctly predict the in vivo genotoxic (GTX) and carcinogenic (C) properties of test compounds. Toxicogenomics, i.e. mRNA-profiling, has been proven successful in improving the prediction of toxicity and understanding of the toxicological mechanisms. Recently, microRNAs have been discovered as post-transcriptional regulators of mRNAs. It is thus hypothesized that using microRNA response-patterns may further improve current prediction methods. This study aimed at predicting genotoxicity and (non-genotoxic) carcinogenicity in vivo, by comparing microRNA- and mRNA-based prediction results, using a frequently applied in vitro liver model and exposing this to a range of well-chosen prototypical carcinogens. Primary mouse hepatocytes (PMH) were treated for 24 and $48 \mathrm{~h}$ with 21 compounds e.g. genotoxic (GTX) and non-genotoxic (NGTX) carcinogens as well as noncarcinogens (NC)). MicroRNA and mRNA expression changes were analyzed by means of Exiqon and Affymetrix microarray-platforms, respectively. Classification was performed by using Prediction Analysis for Microarrays (PAM). Compounds were randomly assigned to the training and validation sets, followed by 10 permutations. Before prediction analysis, preselection of microRNAs as well as mRNAs was performed by use of a leave-one-out t-test. No microRNA sets could be identified that accurately predicted genotoxicity or (non-genotoxic) carcinogenicity in vivo. However, mRNAs could be detected after $24 \mathrm{~h}$ (7) as well as after $48 \mathrm{~h}$ (2) of exposure which appeared reliable in predicting genotoxicity in vivo (accuracy: 90 and 93\%, sensitivity: 65 and 75\%, specificity: 100 and 100\% for the test sets of $24 \mathrm{~h}$ and $48 \mathrm{~h}$ of exposure respectively). From the test compounds, tributylinoxide and para-Cresidine were misclassified. Also, mRNAs were identified capable of classifying NGTXC after 24h (5) as well as after 48h (3) (accuracy: 78 and 88\%, sensitivity: 83 and 83\%, specificity: 75 and 93\%). Despite the relatively high accuracy and sensitivity of our prediction model, Wy-14,643, phenobarbital and ampicillin trihydrate were misclassified. We conclude that genotoxicity and (non-genotoxic) carcinogenicity probably cannot be accurately predicted based on microRNA profiles. In our study, transcript-based prediction analyses clearly outperform microRNAbased analyses. However, a wider range of prototypical compounds would need to be investigated for further validating these findings on the predictive capacity for genotoxicity and carcinogenicity of microRNA expression profiles in vitro. 


\section{Introduction}

Carcinogenic chemicals are substances which may, via ingestion, skin permeation, inhalation or injection, induce the formation of tumors, increase the incidence of malignancy or shorten the time of tumor development. Carcinogenesis comprises a multi-step process which includes the transition of normal cells into cancer cells via a sequence of biological events and characteristic stages. This multi-step process is strongly influenced by internal factors, e.g. genetics and age, but also by external factors e.g. diet, life style and environment, thus causing exposure to carcinogenic agents. Human carcinogens are classified by several authorities such as the International Agency for Research on Cancer (IARC) ${ }^{1}$, the US National Toxicology Program (NTP) ${ }^{2}$ and the US Environmental Protection Agency (EPA) ${ }^{3}$.

Traditionally, carcinogens are divided into two classes, which is based on their modes of action (MOA). Genotoxic carcinogens (GTXC) exert their carcinogenic effect via interactions with the DNA, leading to mutations and to genomic instability. Non-genotoxic carcinogens (NGTXC) act via a range of other mechanisms which do not cause alterations in the DNA sequence.

The carcinogenicity testing strategy initially involves a battery of in vitro genotoxicity tests (e.g. bacterial Ames mutagenicity test ${ }^{4}$, the mouse lymphoma mutagen assay ${ }^{5}$, the micronucleus test ${ }^{6}$ and the chromosomal aberration test ${ }^{7}$ ). In case of a positive outcome, further in vivo verification in lifetime rodent bioassays for genotoxicity and carcinogenicity is mandatory 8,9 . The current in vitro genotoxicity tests are however characterized by an extremely high false positives rate ${ }^{10,11}$. A false positive in this context is defined as a chemical which yields a positive result in the in vitro assay whereas it is negative for genotoxicity in an in vivo assay ${ }^{10}$. Another disadvantage of the current battery of in vitro genotoxicity tests is that it is incapable of detecting the group of NGTXC (false negative result). For chemicals with positive in vivo genotoxicity results, it is decided on a case-by-case basis whether a classic 2-year rodent bioassay is needed to further test the carcinogenic properties of the compound. Such tests are, however, very time-consuming and costly. Moreover, they require the sacrifice of many animals and, more importantly, lack sufficient accuracy for predicting human carcinogenicity (sensitivity $\sim 50 \%{ }^{12}$. Some carcinogens are only carcinogenic in rodents, representing a false positive finding, while other known human carcinogens are not positive in the lifetime rodent bioassay at all, representing a false negative finding ${ }^{12,13}$. This potentially allows widespread human exposure to misclassified chemicals. For these reasons, alternative in vitro test systems are required which are; 1) able to distinguish GTX from NGTX compounds, 2) capable to sensitively identify GTXC and 3) accurate in detecting NGTXC. This could ultimately lead to the development of novel in vitro tests, which are predictive for genotoxicity or carcinogenicity in vivo.

In recent years, the focus of human risk assessment has shifted towards a systems biology approach. By studying different layers of gene regulation, more insight might be acquired into the mechanisms of GTX and NGTX carcinogens. Especially within the field of toxicogenomics, this obtained knowledge might be used to significantly improve the current prediction of human carcinogenicity ${ }^{14}$. By studying for instance carcinogen-induced transcriptome changes, in which the expression of thousands of genes are measured in parallel, new insights might be gained on the potential mechanisms of toxicity of chemical compounds..

Several attempts have already been made to develop in vivo ${ }^{15,16}$ as well as in vitro 17-21 transcriptomics-based prediction tests. A successful study was performed by Magkoufopoulou et al. ${ }^{18}$. In this study, the Prediction Analysis for Microarrays (PAM) tool was used with the 
goal of developing a transcriptomics-based in vitro prediction assay for in vivo genotoxicity. Transcriptomics changes induced in the human liver cell line HepG2 by 34 compounds after treatment for 12, 24, and $48 \mathrm{~h}$ were evaluated for the selection of gene-sets that are capable of discriminating between in vivo GTX and in vivo NGTX. These models were validated by using an additional set of 28 chemicals. Magkoufopoulou et al. proposed the combination of the classical bacterial gene mutation assay according to Ames with in vitro transcriptomics, from chemically treated HepG2 for 24h, as an upgraded in vitro approach for predicting in vivo genotoxicity of chemicals ( $89 \%$ accuracy, $91 \%$ sensitivity and $87 \%$ specificity).

Another interesting field within the -omics based chemical risk assessment domain is the studying of the microRNAome. MicroRNAs are non-coding regulatory RNA molecules $(\sim 22$ nucleotides) that bind multiple target messenger RNAs (mRNA) in their 3' untranslated region (3'-UTR) and thereby suppress the translation into proteins ${ }^{22,23}$. The result is silencing of the target mRNA. Dysregulated expression of microRNAs has been previously observed in human cancer tissues e.g. hepatocellular carcinoma (HCC), and seems promising for defining tumor status ${ }^{24-27}$. However, microRNA expression alterations might already occur before the onset of HCC in healthy individuals and may therefore serve as early biomarkers of genotoxicity and carcinogenicity following environmental carcinogen exposure. In order to provide useful biomarkers in human risk assessment it is, however, required, that these microRNA expression changes persistently occur and thus are irreversible ${ }^{28-30}$. When an organism is exposed to a xenobiotic compound, cells respond by altering the pattern of gene expression, including microRNAs ${ }^{30}$. Altered microRNA expression affects protein translation, which in turn alters cellular physiology causing adverse biological effects. Moreover, different types of cellular stress have been shown to affect microRNA expression. Short term exposure mainly induces microRNAs which play a role in mechanisms of adaptation or tolerance to stress factors, in order to survive ${ }^{30}$. Following long term exposure the behavior of these microRNAs may change to a more tumor-suppressive or oncogenic nature.

Consequently, between similar chemicals, a certain degree of overlap in their microRNA response is expected ${ }^{30-32}$. In a study by Koufaris et al ${ }^{32}$, an attempt was made to distinguish classes of genotoxic and non-genotoxic (or epigenetic) hepatocarcinogens from nonhepatocarcinogens based on their microRNA expression pattern (i.e. based on the expression of miR-200a/200b/429, miR-96/182 clusters and miR-34a) after 3 months of exposure in an in vivo rat model. They were able to associate these hepatic microRNA profiles with the mode of action of the chemical treatments and with carcinogenic mechanisms leading to HCC. In a recent in vitro study ${ }^{31}$, we were able to show that the expression of mmu-miR-503-5p in primary mouse hepatocytes (PMH) exposed for $48 \mathrm{~h}$ to GTX carcinogens, is distinct from PMH treated with NGTX carcinogens. However, in that study, we were not able to use this discriminative microRNA for qualitative classification purposes due to the limited number of compounds within each class (3 GTXC and 3NGTXC).

Since there is a high demand for developing alternative in vitro test systems to accurately predict genotoxicity in vivo and non-genotoxic carcinogenicity, we aimed, in this current study, at discriminating in vivo GTX from NGTX compounds, and NGTXC from NC using in vitro microRNA profiles, and at evaluating larger sets of compounds. For this purpose, PMH were exposed for $24 \mathrm{~h}$ and $48 \mathrm{~h}$ to 21 compounds belonging to either the GTX resp. NGTX classes or NGTXC resp. NC classes. Whole genome gene expression and microRNA expression changes were analyzed by means of Affymetrix and Exiqon microarray-platforms respectively. Classification was again performed by using Prediction Analysis for Microarrays (PAM). By randomly assigning the compounds to training and validation sets, performing permutations and pre-selecting mRNAs and microRNAs based on a leave-one-out t-test, consistent results 
were obtained. MicroRNA-based prediction analysis of compounds was compared with mRNA-based results. The obtained results were then investigated in more detail with respect to their potential significance for human cancer risk assessment.

\section{Materials and methods}

\section{Chemical characteristics}

Seventeen chemicals were obtained through Sigma-Aldrich, TCDD through Cerilliant, DMBA through ICN, E2 through Steraloids Inc. and PhB through Brocacef Intramuraal bv (see Table 4.1). Genotoxicity characterization of these compounds is based on information from in vitro tests (Ames mutagenicity test ${ }^{4,7}$, mouse lymphoma mutagen assay (MLA) ${ }^{33}$, and the combination of in vitro Chromosomal Aberration and Micronucleus test (CA/MN) ${ }^{6,34-48}$ ) as well as from in vivo assays ( $\mathrm{MN}{ }^{49}$, CA, transgenic rodent assay (TRA) ${ }^{11,50-52}$ ). According to a report by the Food and Drug Administration (FDA) (34), a compound is considered GTX in vitro or in vivo when at least one in vitro or in vivo genotoxicity assay showed clear positive results (see Table 4.1). Carcinogenicity assessment of these compounds is based on human carcinogen classification information from the International Agency for Research on Cancer (IARC) ( 900 classified compounds; 100 human carcinogens) ${ }^{1}$, National Toxicology Program (NTP) ( 240 chemicals) ${ }^{2}$, Environmental Protection Agency (EPA) ( $\sim 50$ chemicals) 3 , Toxicological Data Network (TOX-net) (information on $>400,000$ chemicals) ${ }^{53}$ or on other available literature on carcinogenicity assays ${ }^{54}$. A compound is considered a human carcinogen when at least one of these sources defines it as carcinogenic to humans. Carcinogenicity subclassification of a compound was then based on the outcome of the in vivo genotoxicity tests together with the human carcinogenicity assessments (see Table 4.1).

\section{Cell culture, dose determination and chemical treatment}

Primary mouse hepatocytes (PMH) were isolated from adult male C57BL/6 mice using a perfusion method, and cultured between a collagen-collagen sandwich formation in two 6 wells plates, as described before ${ }^{31,55-57}$. After a recovery period of 40-42h, the Dulbecco's Modified Eagle's (Gibco BRL, Breda, The Netherlands) culture medium was replaced by culture medium containing either of the 21 selected test compounds or a vehicle control $(0.5 \%$ of DMSO, PBS or $\mathrm{EtOH})$. A dose-response experiment was performed using the MTT assay ${ }^{58}$ with $24 \mathrm{~h}$ exposure in order to establish a minimally cytotoxic dose resulting in $\pm 80 \%$ viability (data not shown). Ultimately, PMH were treated with the IC20-24h of each chemical (as shown in Table 4.1) or with its respective vehicle control, for 24 or $48 \mathrm{~h}$. Independent triplicate biological experiments with hepatocytes from different mice were obtained at every time point. 







\section{Isolation and quality control of RNA}

After $24 \mathrm{~h}$ and $48 \mathrm{~h}$ of exposure, the medium was removed and PMH were harvested in Qiazol (QIAGEN Benelux B.V., Venlo, The Netherlands). For mRNA and microRNA analysis, total RNA was isolated using a miRNeasy Mini Kit (QIAGEN Benelux B.V., Venlo, The Netherlands) conferring to the manufacturer's protocol including DNase I (Qiagen, Inc) treatment. Following purification, RNA concentrations were measured by means of a NanoDrop ${ }^{\circledR}$ ND-1000 spectrophotometer (Thermo Scientific, Wilmington, USA) at 260 and $280 \mathrm{~nm}$. RNA quality and integrity were determined by applying automated gel electrophoresis on an Agilent 2100 Bioanalyzer system (Agilent Technologies Netherlands B.V., Amstelveen, The Netherlands). Only RNA samples which showed clear $18 \mathrm{~S}$ and $28 \mathrm{~S}$ peaks and a RNA integrity number higher than 8 were used. Samples were stored at $-80^{\circ} \mathrm{C}$ until RNA hybridization.

\section{Measuring microRNA expression via Exiqon arrays}

\section{Labelling and hybridization}

156 total RNA samples were used for microRNA analysis. The miRCURYTM locked-nuclei acid array (LNA), 5th and 7th generation (Exiqon, Denmark) contains probes that detect mature forms of all microRNAs present in miRBase 20.0 (http://www.mirbase.org/) ${ }^{59}$. The 5th and 7th generation Exiqon microRNA microarray contains 9,360 and 14,708 reporters, which represent several control probes and sequences of mature human/mouse/rat specific microRNA (4 technical replicates). In our analysis, 661 unique mouse reporters (measurable on both generation arrays) were used. cDNA preparation, labeling, hybridization, washing, scanning were performed according to Exiqon instructions, as was previously described ${ }^{31}$.

The microRNA data discussed in this publication have been deposited in NCBI's Gene Expression Omnibus ${ }^{60}$ and are accessible through GEO Series accession number GSE72014.

\section{Probe annotation, normalization and data filtering}

The processing of the images, probe annotation, normalization and data filtering was performed as previously described ${ }^{31}$. After filtering, $\log 2$ ratios were calculated between average intensities of the treatment of its respective control.

\section{Measuring gene expression via Affymetrix arrays}

Labelling, hybridization, washing and scanning

Mouse Genome 4302.0 array (45101 probes) genechips from Affymetrix were used to measure gene expression. Targets preparation, hybridization, washing and scanning was performed as previously described 31,57 . A total of 184 RNA samples was prepared and analyzed on GeneChip arrays (treated and control samples from each time point were in triplicate).

Normalization, quality controls, including scaling factors, average intensities, present calls, background intensities, noise, and raw Q values, appeared to be within acceptable limits for all chips. Hybridization controls BioB, BioC, BioD, and CreX were called present on all chips and yielded the expected increases in intensities.

The mRNA data discussed in this publication have been deposited in NCBI's Gene Expression Omnibus ${ }^{60}$ and are accessible through GEO Series accession number GSE72081. 
$\underline{\text { Re-annotation using custom CDF, normalization, and data filtering }}$

At first, all raw data (CEL files) were imported into R2.15.0 and run through an in-house quality control pipeline (available through ArrayAnalysis.org ${ }^{61}$ ) and then used for statistical analysis. The $\mathrm{R}$ program is freely available for academic use from the Comprehensive $\mathrm{R}$ Archive Network (http://www.cran.r-project.org/). Then, probe sets were re-annotated by use of custom Cell Definition Files (version 17.0.0. http://brainarray.mbni.med.umich.edu/Brainarray/Database/CustomCDF/17.0.0/entrezg.as p). After re-annotation, data were normalized by Robust Multichip Average normalization and $\log 2$ transformed using the R package "affy". Low expressed mRNAs were filtered out by using a $\log [$ intensity cutoff] $>5$. This cutoff was based on the 1 st quartile of the distribution of average intensities of all reporters. Filtering out low expressed mRNAs reduces the amount of noise being measured and thus excludes non-informative mRNAs 62,63. After the low expression mRNAs were filtered out a list of 11,029 unique gene IDs remained for further analysis. $\log 2$ ratios were then calculated based on average intensities (after filtering) between the treated and vehicle control samples.

\section{Identifying DE-miRs and DEGs for predicting in vivo genotoxicity and non-genotoxic carcinogenicity}

\section{Selecting discriminative DE-miRs and DEGs}

A selection of classifying microRNAs and mRNAs was identified by using the triplicate (biological replicates) control-corrected $\log 2$ ratios after $24 \mathrm{~h}$ and $48 \mathrm{~h}$ of compound administration separately. The goal of this exercise was to identify microRNAs and mRNAs which were able to discriminate between: Approach 1) in vivo genotoxic (GTX) resp. nongenotoxic (NGTX) compounds and; Approach 2) in vivo non-genotoxic carcinogens (NGTXC) and non-carcinogens (NC) (see Figure-4.1 for the step-by-step selection method).

In order to identify differentially expressed microRNAs (DE-miRs) and differentially expressed genes (DEGs) able to discriminate between in vivo GTX and NGTX compounds, the set of 21 compounds were divided into GTX resp. NGTX compounds (based on available information on in vitro and in vivo genotoxicity and human carcinogenicity). For the selection of DE-miRs and DEGs capable of discriminating between NGTX-C and NC, a set of 15 compounds was used. 
1) Discriminating between GTX and NGTX



2) Discriminating between NGTX-C and NC



Figure 4.1: Step-by-step selection method to identify differentially expressed genes (DEGs) and differentially expressed microRNAs (DE-miRs) able discriminate between 1) in vivo genotoxic (GTX) and non-genotoxic (NGTX) compounds and 2) in vivo non-genotoxic carcinogens (NGTX-C) and non-carcinogens (NC), in primary mouse hepatocytes $(\mathrm{PMH})$, with the purpose of using them for chemical class prediction within Prediction Analysis for Microarrays (PAM).

For the development of a predictive model, a training set and a test set are required. The training set is used to train the model and the test sets is then used to validate whether the model works. To avoid selection bias of DE-miRs or DEGs, several permutations were performed in which the chemicals from each class were randomly assigned to the training or the test set, thereby keeping the size of the training and test set, and the number of compounds of each class within each set, equal for every permutation. Every training set always contained twice the number of compounds of the test set (14 in training set and 7 in test set for GTX vs NGTX and 10 in training set and 5 in test set for NGTXC vs NC). This random selection was executed by means of the randomization function within Microsoft Excel (see part 1 and 2 of Table 4.2 for the ten different training and test sets of the GTX resp. NGTX compounds, and of the NGTX-C resp. NC.

The goal of this exercise was to identify DE-miRs or DEGs which are able to discriminate between two classes of compounds, therefore, compound-specific effects should be ruled out. Consequently, for the 10 different sets of training compounds, a leave-one-out t-test was performed in order to remove compound-specific DE-miRs and DEGs from the list of potential classifiers. In a leave-one-out t-test, a p-value is calculated between the two classes of compounds within the training set. Every time a p-value is calculated, one of the compounds (=three biological replicate $\log 2$ ratios) within a specific class within the training set is left out of the analysis. For each leave-one-out t-test a p-value is determined for each microRNA or mRNA from the list of potential classifiers. In order to identify discriminating DE-miRs and 
DEGs to separate GTX from NGTX compounds, 14 p-values were obtained. Likewise 10 pvalues were obtained to separate NGTXC from NC. At first, only those differentially expressed microRNAs (DE-miRs) and mRNAs (DEGs) were selected that had a p-value $<0.01$ for each performed leave-one-out t-test representing only those which are specific for a particular class of compounds. Successfully applying a leave-one-out t-test prior to prediction analysis within PAM has been described by others 18,19,31.

Only those DE-miRs and DEGs which were identified in all ten different training sets upon combining results from the permutation tests and the leave-one-out tests, were used for prediction analysis within PAM. PAM (Prediction Analysis for Microarrays, Stanford University Software, version 2.21) is a frequently applied "nearest shrunken centroid" class prediction program 18,19. Training and cross-validation was performed within PAM with the ten different training sets and an appropriate threshold (low misclassification error with a small number of microRNAs and mRNAs) was determined. For each of the 10 training sets, the accuracy, sensitivity and specificity were calculated for predicting the classes of the compounds within the test set. The accuracy is defined as the sum of correct classifications divided by the total number of classifications. The sensitivity is the proportion of positives (approach 1: GTX, approach 2: NGTX-C) correctly classified. The specificity is the proportion of negatives (approach 1: NGTX, approach 2: NC) correctly classified. Finally, average values for accuracy, sensitivity and specificity were calculated taking all 10 training and test sets into account. Triplicate $\log 2$ ratios of the discriminating DE-miRs or DEGs, capable of separating GTX from NGTX compounds and NGTXC from NC, of the $24 \mathrm{~h}$ and $48 \mathrm{~h}$ exposure period were then visualized by means of two-dimensional hierarchical clusters (Pearson correlation, Pairwise complete-linkage) using Gene Pattern (version 3.9.1, http://genepattern.broadinstitute.org $\left.{ }^{64}\right)$. The resulting DE-miRs or DEGs were then further investigated with respect to their biological significance using information from Gene Cards (http://www.genecards.org/) ${ }^{65}$. 


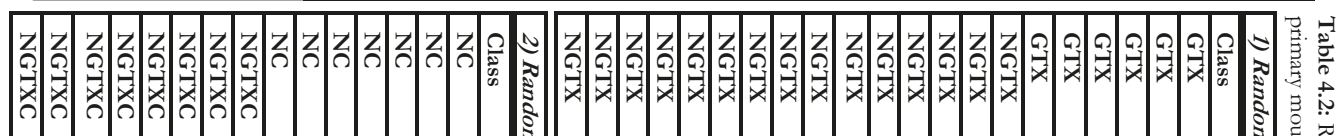
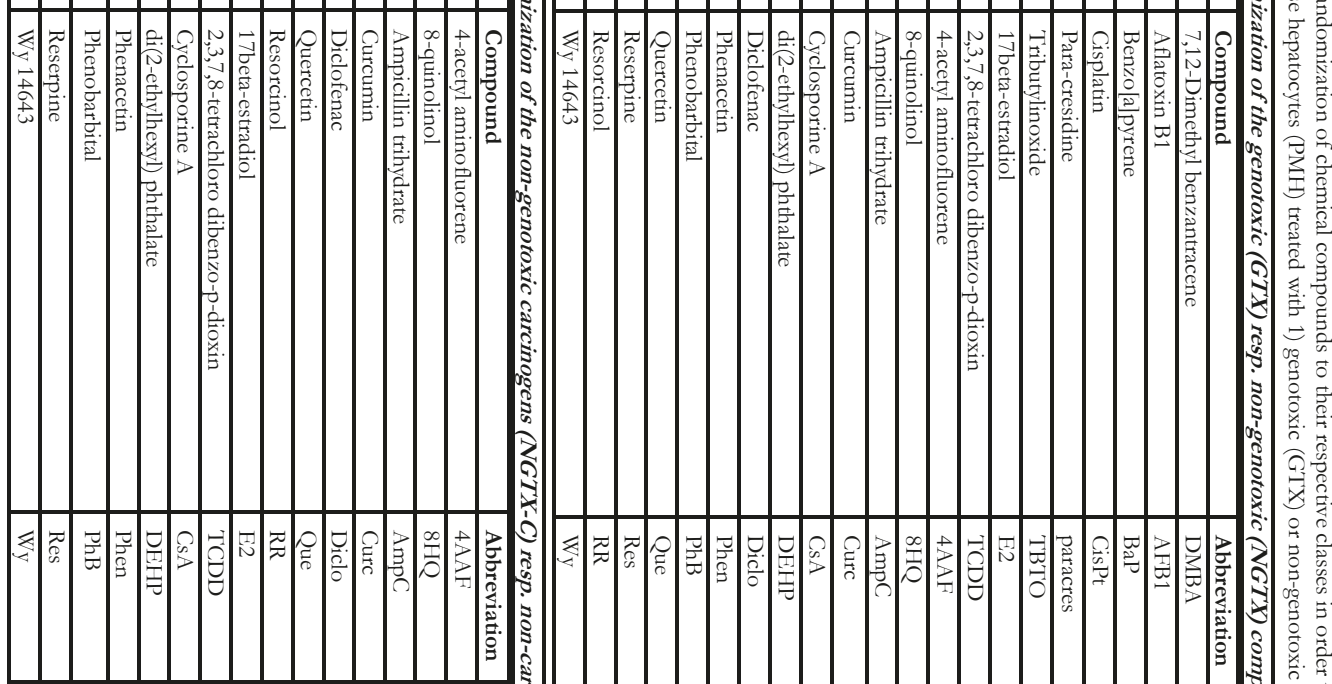

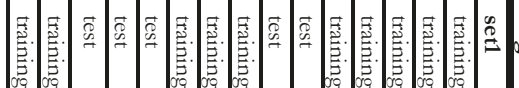

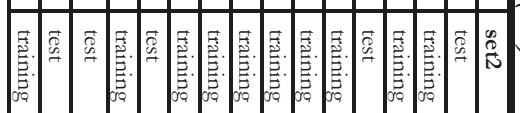

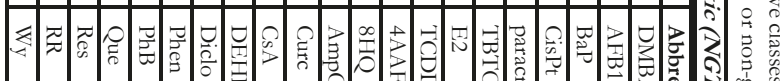

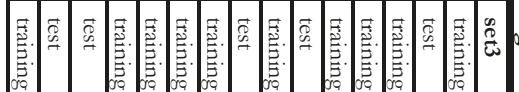

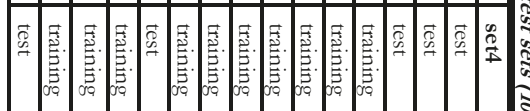

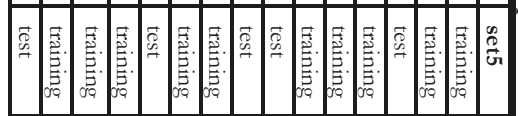

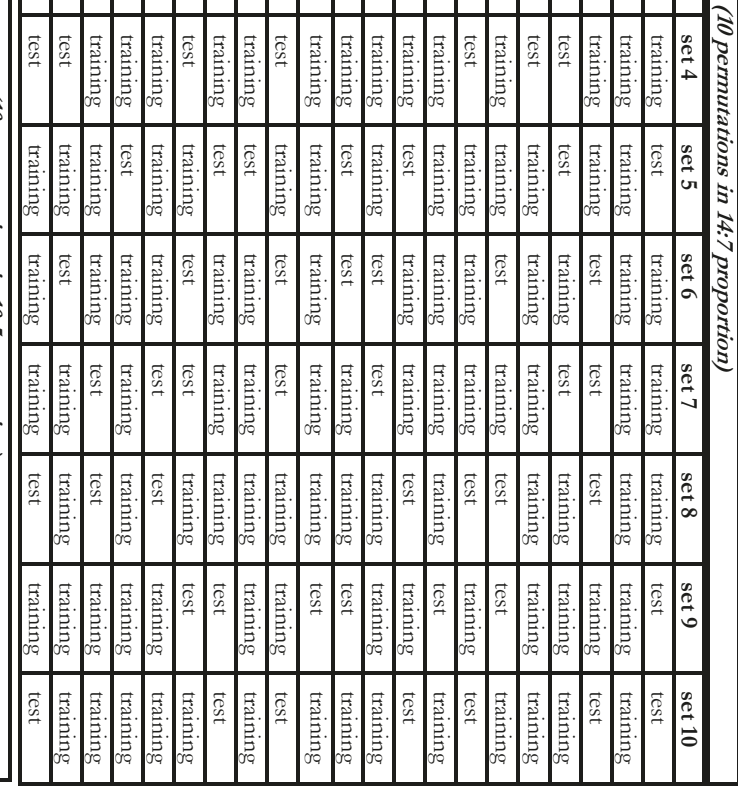

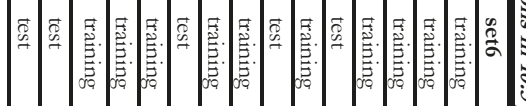

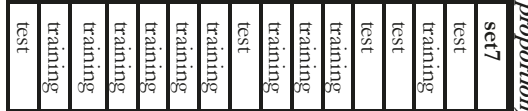

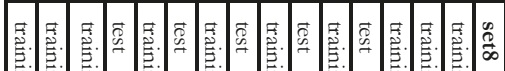

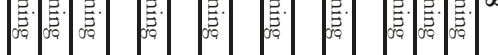

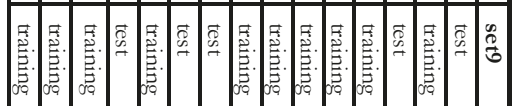

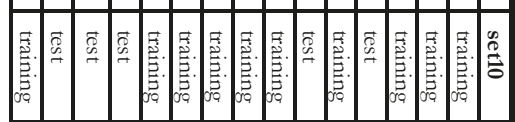




\section{Results}

\section{Identifying classifying microRNAs using a leave-one-out t-test}

\section{Characterizing discriminating microRNAs between GTX vs. NGTX compounds}

After $24 \mathrm{~h}$ of exposure to GTX or NGTX compounds, only 3 DE-miRs could be identified which were present in 2 out of 10 different training sets. This indicates that the majority of microRNA expression patterns between the different compounds are compound-specific (see part A of Supplementary Table 4.1). After 48h, only 2 DE-miRs were commonly selected among 5 out of 10 different training sets (see part B of Supplementary Table 4.1). Based on the lack of overlap between DE-miRs for discriminating GTX from NGTX compounds, we might conclude that microRNA expression patterns are rather compound-specific and are not capable of separating the two classes of compounds.

\section{Identifying discriminating microRNAs between NGTX-C vs. NC compounds}

After $24 \mathrm{~h}$ and $48 \mathrm{~h}$ of exposure to NGTXC or NC compounds, only 2 respectively 1 DE-miRs were identified which were selected in 2 out of 10 different training sets (see part C and D of Supplementary Table 4.1). Also for the purpose of separating NGTXC from NC compounds we may conclude that this is not possible with the current set of compounds due to the fact that microRNA expression patterns seem to be fairly compound-specific.

\section{Identifying classifying mRNAs using a leave-one-out t-test}

\section{Determination of classifying mRNAs between GTX vs. NGTX compounds}

In contrast to the lack of discriminating DE-miRs, sufficient distinct DEGs for classification could be identified in PMH treated for 24h, which are capable of differentiating GTX from NGTX compounds (see part A of Table 4.3). For each of the ten different training sets, DEGs could be identified which are able to discriminate PMH treated with GTX from the ones exposed to NGTX compounds (see part A of Supplementary Table 4.2). After 24h of compound treatment, 7 DEGs (in 8 out of 10 different training sets, thus representing a fairly robust expression profile) were identified, e.g. cordon-bleu WH2 repeat (Cobl - 12808), cryptochrome 1 (photolyase-like) (Cry1 - 12952), DIS3 mitotic control homolog (S. cerevisiae)-like 2 (Dis312 - 208718), GDP-mannose 4, 6-dehydratase (Gmds - 218138), ELKS/RAB6-interacting/CAST family member 2 (Erc2 - 238988), zinc finger protein 866 (Zfp866 - 330788) and calmodulin-lysine N-methyltransferase (Camkmt - 73582) (see part A of Table 4.4 and part A of Supplementary Table 4.2). The triplicate log2 expression values of the 7 DEGs were visualized by means of a hierarchical clustering and a heatmap depicted in part A of Figure 4.2 (for $\log 2$ expression values see part A of Supplementary Table 4.3). Prediction analysis within PAM was performed separately for each of the ten different training sets and in addition accuracy, sensitivity and specificity percentages were calculated for each of the ten different test sets. In the end, these percentages of each of the different training and test sets were averaged. Prediction with these 7 DEGs resulted on average in $91 \%$ accuracy, $68 \%$ sensitivity and $100 \%$ specificity for the training set. For the test set an average of $90 \%$ accuracy, $65 \%$ sensitivity and $100 \%$ specificity could be achieved. Two false negative findings (True class: GTX, Predicted class: NGTX) are para-Cresidine and tributyltinoxide (TBTO) which are both misclassified in 10 out of 10 different test sets. The biological function of the 7 DEGs found after $24 \mathrm{~h}$ of exposure is described in part A of Table 4.4 and is based on information provided by Gene Cards ${ }^{65}$. After $48 \mathrm{~h}$ of exposure to GTX or NGTX compounds, 2 DEGs (consistently found in 10 out of 10 different training sets) could be identified which were capable of separating the two groups of compounds (see part B of Table 
4.3). These DEGs included fragile histidine triad gene (Fhit - 14198) and Ca2+dependent activator protein for secretion 2 (Cadps 2 - 320405) (see part B of Table 4.4 and part B of Supplementary Table 4.2). The triplicate $\log 2$ expression values of these two DEGs, capable of separating PMH treated with GTX from the ones exposed to NGTX compounds, are visualized by means of an hierarchical clustering and a heatmap depicted in part B of Figure 4.2 (for $\log 2$ expression values see part B of Supplementary Table 4.3). An average of $96 \%$ accuracy, $85 \%$ sensitivity and 100\% specificity of the training set could be achieved. More importantly, an average of $93 \%$ accuracy, $75 \%$ sensitivity and 100\% specificity could be obtained for the overall test set. For $48 \mathrm{~h}$, there were also some misclassified (false negative) compounds, namely tributyltinoxide (TBTO) which was misclassified in 10 out of 10 different test sets (True class: GTX, Predicted class: NGTX). The biological role of the 2 DEGs obtained after $48 \mathrm{~h}$ of treatment is described in part B of Table 4.4 using information from Gene Cards ${ }^{65}$.

\section{GTX vs. NGTX compounds}
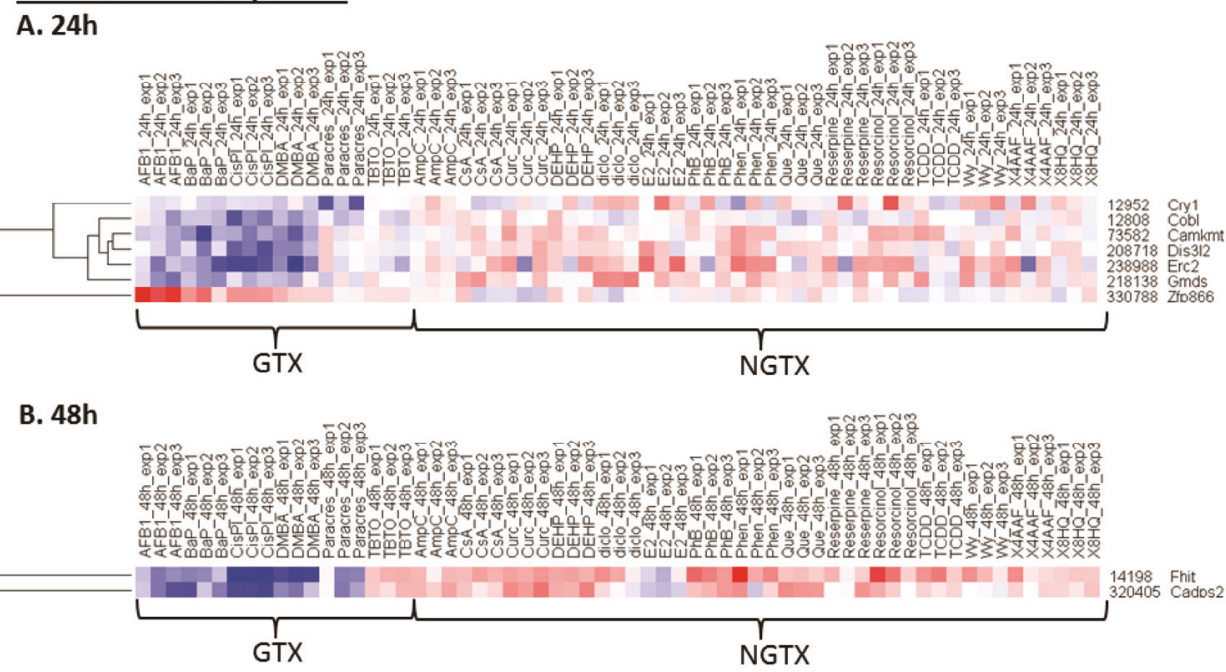

Figure 4.2: Visualization of DEGs able to distinguish primary mouse hepatocytes (PMH) treated for (A) 24h and (B) $48 \mathrm{~h}$ with GTX or NGTX compounds (GTX=genotoxic, NGTX=non-genotoxic, red=upregulation, blue $=$ downregulation).

Table 4.3: Performance of the prediction model for in vivo genotoxicity using differentially expressed genes (DEGs) after (A) $24 \mathrm{~h}$ or (B) $48 \mathrm{~h}$ of exposure in primary mouse hepatocytes $(\mathrm{PMH})($ Acc. $=$ accuracy, Sens. $=$ sensitivity, Spec. $=$ specificity, missclass. error $=$ misclassification error)

\begin{tabular}{|c|c|c|c|c|c|c|c|c|}
\hline \multicolumn{9}{|l|}{ A. } \\
\hline $24 \mathrm{~h}$ & \multicolumn{5}{|c|}{ TRAINING SET } & \multicolumn{3}{|c|}{ TEST SET } \\
\hline Randomization set & Acc. & Sens. & Spec. & misclass. error & Common no. of genes & Acc. & Sens. & Spec. \\
\hline AVG & $91 \%$ & $68 \%$ & $100 \%$ & 0.079 & 7 & $90 \%$ & $65 \%$ & $100 \%$ \\
\hline \multicolumn{9}{|l|}{ B. } \\
\hline $48 \mathrm{~h}$ & \multicolumn{4}{|c|}{ TRAINING SET } & & \multicolumn{3}{|c|}{ TEST SET } \\
\hline Randomization set & Acc. & Sens. & Spec. & misclass. error & Common no. of genes & Acc. & Sens. & Spec. \\
\hline AVG & $96 \%$ & $85 \%$ & $100 \%$ & 0.090 & 2 & $93 \%$ & $75 \%$ & $100 \%$ \\
\hline
\end{tabular}


Table 4.4: List of differentially expressed genes (DEGs) for in vivo genotoxicity prediction, after (A) $24 \mathrm{~h}$ or (B) $48 \mathrm{~h}$ of exposure in primary mouse hepatocytes $(\mathrm{PMH})$.

\begin{tabular}{|c|c|c|c|c|}
\hline A. & & & & \\
\hline \multicolumn{5}{|l|}{$24 \mathrm{~h}$} \\
\hline $\begin{array}{c}\text { Entrez } \\
\text { gene ID }\end{array}$ & $\begin{array}{l}\text { Gene } \\
\text { symbol }\end{array}$ & Gene description & Function & $\begin{array}{l}\text { \# different } \\
\text { training sets } \\
\text { classifying } \\
\text { DEG present }\end{array}$ \\
\hline 73582 & Camkmt & $\begin{array}{l}\text { calmodulin-lysine N- } \\
\text { methyltransferase }\end{array}$ & Methyltransferase & $8 / 10$ \\
\hline 218138 & Gmds & $\begin{array}{l}\text { GDP-mannose 4, 6- } \\
\text { dehydratase }\end{array}$ & $\begin{array}{l}\text { Inhibits poly (ADP-ribose) polymerase } \\
\text { (PARP) activity }\end{array}$ & $8 / 10$ \\
\hline 238988 & Erc2 & $\begin{array}{l}\text { ELKS/RAB6- } \\
\text { interacting/CAST } \\
\text { family member } 2\end{array}$ & Organization of the cytomatrix & $8 / 10$ \\
\hline 330788 & Zfp866 & $\begin{array}{l}\text { zinc finger protein } \\
\qquad 866\end{array}$ & & $8 / 10$ \\
\hline 208718 & Dis 312 & $\begin{array}{l}\text { DIS3 mitotic control } \\
\text { homolog }\end{array}$ & RNA degradation (mRNA and microRNA) & $8 / 10$ \\
\hline 12808 & Cobl & $\begin{array}{l}\text { cordon-bleu WH2 } \\
\text { repeat }\end{array}$ & Regulators of actin dynamics & $8 / 10$ \\
\hline 12952 & Cry1 & $\begin{array}{l}\text { cryptochrome } 1 \\
\text { (photolyase-like) }\end{array}$ & Regulates CLOCK genes & $8 / 10$ \\
\hline \multicolumn{5}{|l|}{ B. } \\
\hline \multicolumn{5}{|l|}{$48 \mathrm{~h}$} \\
\hline $\begin{array}{c}\text { Entrez } \\
\text { gene ID }\end{array}$ & $\begin{array}{l}\text { Gene } \\
\text { symbol }\end{array}$ & Gene description & Function & $\begin{array}{l}\text { \# different } \\
\text { training sets } \\
\text { classifying } \\
\text { DEG present }\end{array}$ \\
\hline 14198 & Fhit & $\begin{array}{l}\text { fragile histidine triad } \\
\text { gene }\end{array}$ & $\begin{array}{l}\text { Modulates the DNA damage checkpoint } \\
\text { response and has a role in the regulation of } \\
\text { apoptosis and cell cycle }\end{array}$ & $10 / 10$ \\
\hline 320405 & Cadps2 & $\begin{array}{l}\mathrm{Ca} 2+\text {-dependent } \\
\text { activator protein for } \\
\text { secretion } 2\end{array}$ & $\begin{array}{l}\text { Phospholipid binding, involved in } \\
\text { exocytosis of vesicles }\end{array}$ & $10 / 10$ \\
\hline
\end{tabular}

\section{Detecting classifying mRNAs between NGTX-C vs. NC compounds}

Although no DE-miRS could be identified which are capable of separating PMH treated with NGTXC from cells exposed to NC, DEGs could be identified after $24 \mathrm{~h}$ of exposure which were actually able to meet these criteria (see part A of Table 4.5). Discriminative DEGs could be identified for the ten different training sets separately. After $24 \mathrm{~h}$ of treatment, 5 DEGs could be identified which were selected within at least 9 out of 10 different training sets. These 5 DEGs were synapse defective 1, Rho GTPase, homolog 1 (C. elegans) (Syde1 - 71709), nidogen 1 (Nid1 - 18073), N-myc downstream regulated gene 3 (Ndrg3 - 29812), dishevelled associated activator of morphomRNAsis 1 (Daam1 - 208846) and small G protein signaling modulator 2 (Sgsm2 - 97761) (see part A of Table 4.6). The triplicate log2 expression values of these 5 DEGs were visualized by means of a hierarchical clustering and a heatmap depicted in 
part A of Figure 4.3 (for $\log 2$ expression values see part $C$ of Supplementary Table 4.3). An average of $91 \%$ accuracy, $92 \%$ sensitivity and $90 \%$ of specificity could be obtained for the training set while an average accuracy of $78 \%$, sensitivity of $83 \%$ and a specificity of $75 \%$ could be obtained for the overall test set. One false positive and one false negative result were obtained; ampicillin trihydrate which was misclassified in 9 out of 10 different test sets (True class: NC, Predicted class: NGTX-C $\rightarrow$ false positive) and phenobarbital which was misclassified in 8 out of 10 test sets (True class: NGTX-C, Predicted class: NC $\rightarrow$ false negative).

After $48 \mathrm{~h}$ of exposure, 3 DEGs could be identified which were able to differentiate between PMH treated with NGTXC and cells exposed to NC as based on results obtained from 9 out of 10 different training sets (see part B of Table 4.5). These 3 DEGs included cystatin C (Cst3 - 13010), RAB31, member RAS oncogene family (Rab31 - 106572) and F-box protein 2 (Fbxo2 - 230904) (see part B of Table 4.6). The hierarchical clustering and the heatmap, in which triplicate $\log 2$ expression values are visualized, is depicted in part B of Figure 4.3 (for $\log 2$ expression values see part D of Supplementary Table 4.3). For the overall training set an average $94 \%$ of accuracy, $88 \%$ of sensitivity and $100 \%$ specificity could be obtained. The average accuracy, specificity and sensitivity of the test set was $88 \%, 83 \%$ and $93 \%$ respectively. For one compound, Wy 14643, false negative results were obtained, after $48 \mathrm{~h}$ of exposure, as it was misclassified in 9 out of 10 test sets (True class: NGTX-C, Predicted class: NC).
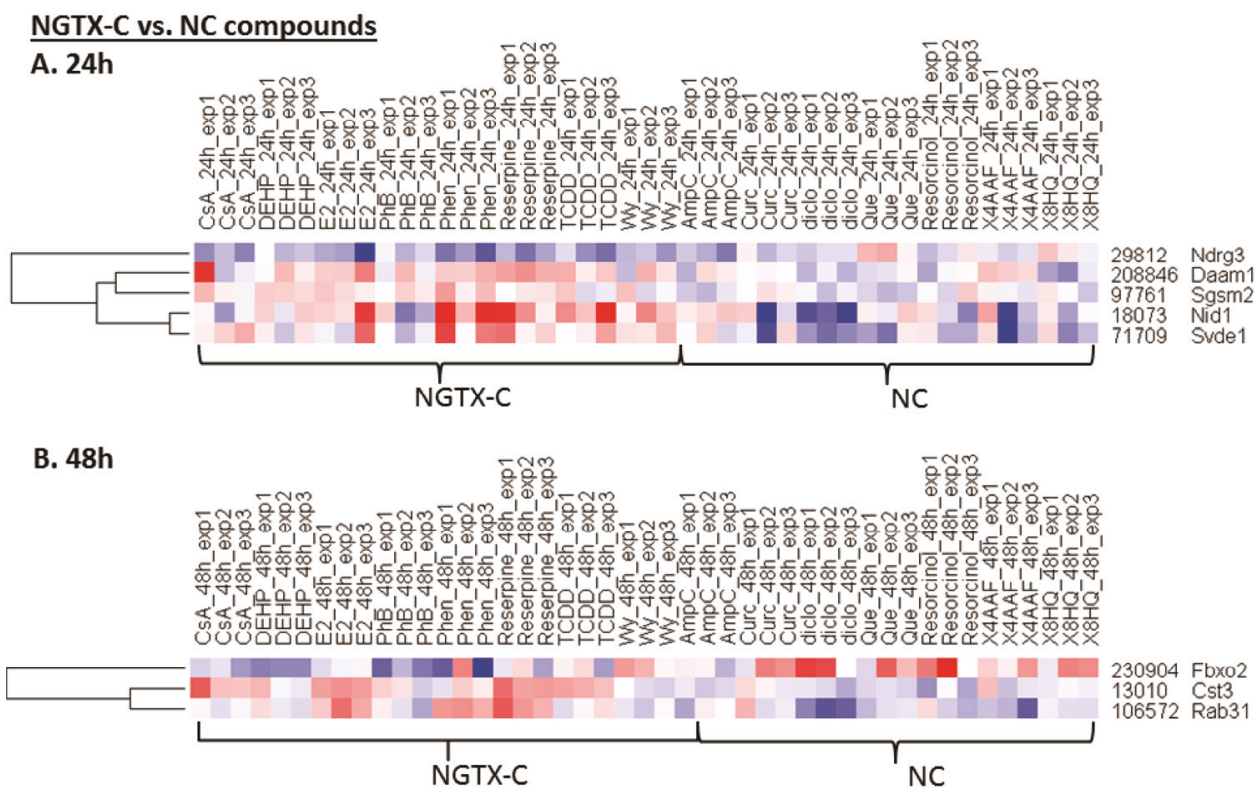

Figure 4.3: Visualization of DEGs able to discriminate primary mouse hepatocytes (PMH) exposed for (A) 24h and (B) $48 \mathrm{~h}$ with NGTX-C or NC compounds (NGTX-C=non-genotoxic carcinogen, NC=non-carcinogen, red=upregulation, blue $=$ downregulation). 
Table 4.5: Performance of the prediction model for in vivo non-genotoxic carcinogenicity using differentially expressed genes (DEGs) after (A) $24 \mathrm{~h}$ or (B) $48 \mathrm{~h}$ of exposure in primary mouse hepatocytes (PMH). (Acc.=accuracy, Sens.=sensitivity, Spec. $=$ specificity, missclass. error=misclassification error $)$



Table 4.6: List of differentially expressed genes (DEGs) for in vivo (non-genotoxic) carcinogenicity prediction, after (A) $24 \mathrm{~h}$ or (B) $48 \mathrm{~h}$ of exposure in primary mouse hepatocytes (PMH).

\begin{tabular}{|c|c|c|c|c|c|}
\hline \multicolumn{6}{|l|}{ A. } \\
\hline $24 \mathrm{~h}$ & & & & & \\
\hline $\begin{array}{l}\text { Entrez } \\
\text { Gene } \\
\text { ID }\end{array}$ & $\begin{array}{l}\text { Gene } \\
\text { Symbol }\end{array}$ & Gene description & Function & Association with cancer & $\begin{array}{l}\text { \# different training } \\
\text { sets classifying } \\
\text { DEG present }\end{array}$ \\
\hline 71709 & Syde1 & $\begin{array}{c}\text { synapse defective 1, } \\
\text { Rho GTPase, } \\
\text { homolog } 1 \text { (C. } \\
\text { elegans) }\end{array}$ & $\begin{array}{l}\text { Activation of Rho } \\
\text { GTPase activity }\end{array}$ & $\begin{array}{l}\text { Used for diagnosis of } \\
\text { prostate cancer }\end{array}$ & $10 / 10$ \\
\hline 18073 & Nid1 & nidogen 1 & $\begin{array}{l}\text { Degradation of the } \\
\text { extracellular matrix, } \\
\text { Laminin interactions }\end{array}$ & $\begin{array}{l}\text { Loss of nidogen expression } \\
\text { may favor invasion and } \\
\text { metastasis of cancer cells }\end{array}$ & $9 / 10$ \\
\hline 29812 & Ndrg3 & $\begin{array}{l}\text { N-myc } \\
\text { downstream } \\
\text { regulated gene } 3\end{array}$ & $\begin{array}{l}\text { Cell differentiation, } \\
\text { Negative regulation of } \\
\text { cell growth }\end{array}$ & $\begin{array}{l}\text { Promotes prostate cancer } \\
\text { cell growth }\end{array}$ & $9 / 10$ \\
\hline 208846 & Daam1 & $\begin{array}{l}\text { dishevelled associated } \\
\text { activator of } \\
\text { morphogenesis } 1\end{array}$ & $\begin{array}{l}\text { Actin binding, } \\
\text { Identical protein } \\
\text { binding, Protein } \\
\text { binding, Rho } \\
\text { GTPase binding, } \\
\text { Wnt signaling }\end{array}$ & $\begin{array}{c}\text { Promotes the migration of } \\
\text { breast cancer cells }\end{array}$ & $9 / 10$ \\
\hline 97761 & Sgsm2 & $\begin{array}{c}\text { small } G \text { protein } \\
\text { signaling modulator } 2\end{array}$ & $\begin{array}{l}\text { Positive regulation of } \\
\text { Rab GTPase activity }\end{array}$ & $\begin{array}{l}\text { Associated with bladder } \\
\text { cancer }\end{array}$ & $9 / 10$ \\
\hline \multicolumn{6}{|l|}{ B. } \\
\hline $\begin{array}{l}48 h \\
\text { Entrez } \\
\text { Gene } \\
\text { ID }\end{array}$ & $\begin{array}{l}\text { Gene } \\
\text { Symbol }\end{array}$ & Gene description & Function & Association with cancer & $\begin{array}{l}\text { \# different training } \\
\text { sets classifying } \\
\text { DEG present }\end{array}$ \\
\hline 13010 & Cst3 & cystatin $\mathrm{C}$ & $\begin{array}{l}\text { cysteine-type } \\
\text { endopeptidase } \\
\text { inhibitor activity, }\end{array}$ & $\begin{array}{c}\text { Increased during cancer } \\
\text { progression }\end{array}$ & $9 / 10$ \\
\hline 106572 & Rab31 & $\begin{array}{l}\text { RAB31, member RAS } \\
\text { oncogene family }\end{array}$ & $\begin{array}{l}\text { protease binding } \\
\text { GDP binding and }\end{array}$ & Affects cancer progression & $9 / 10$ \\
\hline 230904 & Fbxo2 & F-box protein 2 & $\begin{array}{l}\text { GTP binding } \\
\text { Signal transduction } \\
\text { and regulation of the } \\
\text { cell cycle }\end{array}$ & $\begin{array}{c}\text { F-box proteins have a role } \\
\text { in cancer both as tumor } \\
\text { supressor } \\
\text { as well as oncogenes }\end{array}$ & $9 / 10$ \\
\hline
\end{tabular}




\section{Discussion}

In this study, two approaches were followed in parallel in order to identify classifying DE-miRs between GTX vs. NGTX and NGTX-C vs. NC compounds in PMH after treatment for $24 \mathrm{~h}$ and $48 \mathrm{~h}$. We were however not able to identify DE-miRs which were capable of distinguishing between the different sets of compounds. Based on these results we conclude that given the current set of compounds, no reliable in vitro microRNA expression patterns could be retrieved for predicting in vivo characteristics (i.e. genotoxicity or non-genotoxic carcinogenicity).

By contrast, in vitro based mRNA profiles appear to outperform in vitro based microRNA profiles for the purpose of predicting in vivo genotoxicity as well as non-genotoxic carcinogenicity. These results are coherent with a study performed by Melis et al., in 201420 who used in vivo murine hepatic microRNA and mRNA expression signatures for predicting the (non-)genotoxic carcinogenic potential of chemicals. Their study also demonstrated that the in vivo microRNA-ome has less discriminative power than the transcriptome to correctly identify (non-)genotoxic carcinogen classes.

For the identification of discriminative DEGs for predicting in vivo genotoxicity, the best performance was achieved when using overlapping DEGs (between the 10 different training and test sets) of PMH exposed for $48 \mathrm{~h}$. The average accuracy, sensitivity and specificity of the training set in general were $96 \%, 85 \%$ and $100 \%$ and of the overall accuracy, sensitivity and specificity of the test set was $93 \%, 75 \%$ and $100 \%$. Tributyltinoxide (TBTO) was however consistently misclassified as a NGTX compound. In 1987 an extensive evaluation of several in vivo and in vitro short-term tests was performed by Davis and colleagues who showed that TBTO gave negative results in short-term tests using various genetic endpoints ${ }^{51}$. Though negative within short-term studies, at cytotoxic concentrations TBTO seemed mutagenic in one bacterial strain, clastogenic in $\mathrm{CHO}$ cells in vitro and produced micronuclei in mouse bone marrow cells in vivo. Furthermore, they found that TBTO is able to induce morphogenetic differentiation processes in vitro. They concluded that for human risk assessment these biological effects should be taken into account. That this compound is misclassified using the current set of DEGs, is in line with the inconclusive in vitro as well as in vivo genotoxicity results with the battery of traditional tests.

The two DEGs for predicting in vivo genotoxicity, were fragile histidine triad gene (Fhit 14198) and Ca2+dependent activator protein for secretion 2 (Cadps 2 - 320405). These DEGs could be potential candidates for prediction purposes, though this needs to be further validated with more compounds.

Fragile histidine triad gene (Fhit - 14198), downregulated by GTX compounds and upregulated by NGTX compounds (see part B of Figure 4.2), encodes a diadenosine 5',5"'P1,P3-triphosphate hydrolase involved in purine metabolism. Furthermore, it is a gene involved in the modulation of the DNA damage checkpoint response ${ }^{66}$, which has a role in the regulation of apoptosis and cell cycle and is identified as a tumor suppressor gene ${ }^{67}$. Fhit protein expression is essential to protect from DNA damage-induced cancer initiation and progression by modulating genome stability, oxidative stress and levels of accumulating DNA damage. Thus, Fhit, whose expression is lost or reduced in many human cancers, is a tumor suppressor and genome caretaker whose loss initiates genome instability in preneoplastic lesions ${ }^{68}$.

The second DEG, Ca2+dependent activator protein for secretion 2 (Cadps2 - 320405), downregulated by GTX compounds and upregulated by NGTX compounds (see part B of Figure 4.2), is a gene involved in phospholipid binding and exocytosis of vesicles ${ }^{69}$. 
The finding that the expression of Fhit differs between GTX and NGTX compounds, is coherent with the study by Mathijs and colleagues in $2009{ }^{21}$ who identified a set of classifying mRNAs based on a limited set of compounds (four GTX and two NGTX compounds). They concluded that negative expression changes of Fhit by GTX compounds leads to upregulation of Mdm2 ${ }^{21}$. Oncogenic Mdm2, already identified as being able to discriminate between GTX and NGTX compounds in rat livers ${ }^{70}$, binds with p53 thereby avoiding inhibition of cellular proliferation or the induction of cell death ${ }^{21,71}$. Therefore, downregulation of Fhit might lead to overexpression of the $\mathrm{Mdm} 2$ gene which is associated with an increased tumorigenic potential since it can overcome the inhibition of cell growth by p53 ${ }^{21}$. Considering its relevance in the mechanism of GTX compounds, Fhit may be a promising candidate for future research on developing biomarkers for predicting genotoxicity in vivo.

Upon determining classifying DEGs for predicting in vivo non-genotoxic carcinogenicity, the best performance was also achieved when utilizing DEGs of PMH exposed for 48h. The average accuracy, sensitivity and specificity, based on results obtained from the ten different training sets, were $94 \%, 88 \%$ and $100 \%$ for the training set in general and $88 \%, 83 \%$ and $93 \%$ for the overall test set. Wy 14643 (Wy) was however consistently misclassified as being an NC compound while in fact this is a NGTX-C compound. In consideration of this misclassification, it is important to note that the results for this compound from in vivo carcinogenicity studies are not conclusive ${ }^{53}$. Species differences in biologic activity and carcinogenicity exist ${ }^{72}$. The carcinogenicity of $\mathrm{Wy}_{\mathrm{y}}$ also strongly depends on the level of peroxisome proliferator-activated receptor- $\alpha$ (PPAR- $\alpha)$ in the liver which can be different among mouse liver models. Szalowska and colleagues ${ }^{73}$ showed that the occurrence of the full spectrum of PPAR $\alpha$-induced processes such as lipid metabolism, immunity and carcinogenesis exclusively occur at the ex vivo and in vivo level. The explanation the authors gave was that Kupffer cells seem to play a central role in peroxisome proliferator-induced carcinogenesis. Therefore, the carcinogenic effects of Wy--mediated PPAR- $\alpha$ activation may only occur in intact murine liver and not in $\mathrm{PMH}{ }^{74}$.

The 3 DEGs, which were capable of separating PMH treated with NGTXC from the cells that were exposed to NC were cystatin C (Cst3 - 13010), RAB31, member RAS oncogene family (Rab31 - 106572) and F-box protein 2 (Fbxo2 - 230904) (see part B of Table 4.6).

Cystatin C (Cst3 - 13010), upregulated in PMH exposed to NGTX-C compounds and downregulated in $\mathrm{PMH}$ exposed to $\mathrm{NC}$ compounds, is a secreted cysteine protease inhibitor that regulates bone resorption, extracellular matrix proteins, neutrophil chemotaxis, and tissue inflammation, as well as resistance to bacterial and viral infections ${ }^{65,75}$. Cst 3 is ubiquitously expressed and present in most bodily fluids where it inhibits the activities of cathepsins, a family of cysteine proteases that can promote cancer cell invasion and metastasis 76 . Furthermore, it has been reported to have an inhibitory effect of apoptosis caused by caspase- 3 induction ${ }^{77}$. Increased expression of Cst3 is reported during cancer development and progression in e.g. melanoma and colorectal cancer ${ }^{78}$.

RAB31, member RAS oncogene family (Rab31 - 106572), upregulated in PMH exposed to NGTX-C compounds and downregulated in PMH exposed to NC compounds, belongs to the family of small GDP-binding Rab protein which are key regulators of intracellular membrane trafficking; from the formation of transport vesicles within the Golgi apparatus to their fusion with membranes. Rabs cycle between an inactive GDP-bound form and an active GTP-bound form that is able to recruit to membranes different set of downstream effectors directly responsible for vesicle formation, movement, tethering and fusion ${ }^{65}$. Deregulation of Rab proteins was found to contribute to neurological and neurodegenerative diseases, lipid storage 
disorders, and cancer ${ }^{79}$. Recently ${ }^{80}$, it has been reported that overexpression of Rab31 is associated with worse prognosis after liver resection in hepatocellular carcinoma patients. Furthermore, within this specific study ${ }^{80}$, in which also in vitro assays were performed, it was reported that elevated levels of Rab31 increased the $\mathrm{Bcl}-2 / \mathrm{BAX}$ ratio via PI3K/AKT signaling. This decreased cell apoptosis and accelerated hepatocellular carcinoma cell growth consequently.

F-box protein 2 (Fbxo2 - 230904), downregulated in NGTX-C-exposed PMH and upregulated in $\mathrm{NC}$-exposed $\mathrm{PMH}$, is a gene involved in signal transduction and regulation of the cell cycle 81. The F-box proteins constitute one of the four subunits of the ubiquitin protein ligase complex named SCFs (SKP1-cullin-F-box), which function in phosphorylation-dependent ubiquitination. F-box proteins have a role in cancer both as tumor suppressor as well as oncogenes ${ }^{82}$. The exact role of Fbxo2 in cancer, particularly HCC, has not been described however family member Fbxo22 is recently reported to promote hepatocellular carcinoma cell proliferation both in vitro and in vivo ${ }^{83}$.

All three mRNAs seem to play a role in cancer-related events such as the development and progression e.g. of HCC. Therefore, they might be good candidates to be further investigated in future studies focused on developing in vitro-based alternatives for predicting non-genotoxic carcinogenicity in vivo.

To our knowledge, the use of permutations to select training and test sets, was not applied before in identifying mRNAs for the purpose of predicting in vivo genotoxicity 18,21 or carcinogenicity. By using randomized sets of training and test compounds, the performance of the prediction model significantly improves; this already occurs after 10 permutations. This needs to be further validated in future studies on more compounds and permutations thereby taking the identified classifiers for in vivo genotoxicity and non-genotoxic carcinogenicity into account.

\section{Conclusion}

We conclude that in vitro-based prediction analysis of in vivo genotoxicity or (non-genotoxic) carcinogenicity is best performed by exploiting mRNA-based expression profiles instead of microRNA-based models. mRNA profiles retrieved upon $48 \mathrm{~h}$ of genotoxic/carcinogenic exposure, rather than after $24 \mathrm{~h}$ of treatment appear more predictive. Candidates for distinguishing between in vivo GTX and NGTX compounds were identified i.e. Fhit and Cadps2. Especially Fhit has a direct link with DNA damage resulting from GTX exposure. Similarly, for discriminating between in vivo NGTX-C and NC compounds also successful DEGs have been identified i.e. Cst3, Rab31 and Fbxo2. These DEGs have a clear role in cancer-related events e.g. in the development and progression of hepatocellular carcinoma. These gene candidates may be used for future validation studies, considering more compounds, permutations and the use of human cell models, to ultimately improve chemical cancer risk assessment for humans.

\section{Supplementary data}

Supplementary Tables 4.1-4.3 are available upon request. 


\section{References}

1. International Agency for Research on Cancer (IARC). IARC Monographs on the Evaluation of Carcinogenic Risks to Humans. http://monographs.iarc.fr/ENG/Classification/ClassificationsGroupOrder.pdf, (accessed on November 14, 2014) (2014).

2. US Department of Health and Human Services. Public Health Service. National Toxicology Program (NTP). Report on Carcinogens. http://ntp.niehs.nih.gov/pubhealth/roc/roc13/index.html, (accessed on November 14, 2014) (2014).

3. Mills, A. \& Foureman, G.L. US EPA's IRIS pilot program: establishing IRIS as a centralized, peer-reviewed data base with agency consensus. Integrated Risk Information System. Toxicology 127, 85-95 (1998).

4. Ames, B.N., Lee, F.D. \& Durston, W.E. An improved bacterial test system for the detection and classification of mutagens and carcinogens. Proc Natl Acad Sci US A 70, 782-6 (1973).

5. Hozier, J., Sawyer, J., Moore, M., Howard, B. \& Clive, D. Cytogenetic analysis of the L5178Y/TK+/- leads to TK-/- mouse lymphoma mutagenesis assay system. Mutat Res 84, 169-81 (1981).

6. Muller-Tegethoff, K., Kasper, P. \& Muller, L. Evaluation studies on the in vitro rat hepatocyte micronucleus assay. Mutat Res 335, 293-307 (1995).

7. Kirkland, D., Reeve, L., Gatehouse, D. \& Vanparys, P. A core in vitro genotoxicity battery comprising the Ames test plus the in vitro micronucleus test is sufficient to detect rodent carcinogens and in vivo genotoxins. Mutat Res 721, 27-73 (2011).

8. Thybaud, V. et al. Strategy for genotoxicity testing: hazard identification and risk assessment in relation to in vitro testing. Mutat Res 627, 41-58 (2007).

9. Pfuhler, S. et al. Genetic toxicity assessment: employing the best science for human safety evaluation part IV: Recommendation of a working group of the Gesellschaft fuer Umwelt-Mutationsforschung (GUM) for a simple and straightforward approach to genotoxicity testing. Toxicol Sci 97, 237-40 (2007).

10. Kirkland, D. et al. How to reduce false positive results when undertaking in vitro genotoxicity testing and thus avoid unnecessary follow-up animal tests: Report of an ECVAM Workshop. Mutat Res 628, 31-55 (2007).

11. Kirkland, D., Kasper, P., Muller, L., Corvi, R. \& Speit, G. Recommended lists of genotoxic and nongenotoxic chemicals for assessment of the performance of new or improved genotoxicity tests: a follow-up to an ECVAM workshop. Mutat Res 653, 99-108 (2008).

12. Ennever, F.K. \& Lave, L.B. Implications of the lack of accuracy of the lifetime rodent bioassay for predicting human carcinogenicity. Regul Toxicol Pharmacol 38, 52-7 (2003).

13. de Vries, A., van Steeg, H. \& Opperhuizen, A. Transgenic mice as alternatives in carcinogenicity testing: $\begin{array}{lllll}\text { current } & \text { status. } & \text { RIVM } & \text { Available } & \text { at }\end{array}$ http://rivm.openrepository.com/rivm/bitstream/10029/8911/1/340700001.pdf, (accessed July 28, 2015) (2004).

14. Piersma, A.H. et al. A critical appraisal of the process of regulatory implementation of novel in vivo and in vitro methods for chemical hazard and risk assessment. Crit Rev Toxicol 44, 876-94 (2014).

15. Fielden, M.R. et al. Development and evaluation of a genomic signature for the prediction and mechanistic assessment of nongenotoxic hepatocarcinogens in the rat. Toxicol Sci 124, 54-74 (2011).

16. Ellinger-Ziegelbauer, H., Stuart, B., Wahle, B., Bomann, W. \& Ahr, H.J. Comparison of the expression profiles induced by genotoxic and nongenotoxic carcinogens in rat liver. Mutat Res 575, 61-84 (2005).

17. van Delft, J.H. et al. Discrimination of genotoxic from non-genotoxic carcinogens by gene expression profiling. Carcinogenesis 25, 1265-76 (2004).

18. Magkoufopoulou, C. et al. A transcriptomics-based in vitro assay for predicting chemical genotoxicity in vivo. Carcinogenesis (2012).

19. Van den Hof, W.F. et al. Classification of hepatotoxicants using HepG2 cells: A proof of principle study. Chem Res Toxicol 27, 433-42 (2014).

20. Melis, J.P. et al. In vivo murine hepatic microRNA and mRNA expression signatures predicting the (non)genotoxic carcinogenic potential of chemicals. Arch Toxicol (2014).

21. Mathijs, K. et al. Discrimination for genotoxic and nongenotoxic carcinogens by gene expression profiling in primary mouse hepatocytes improves with exposure time. Toxicol Sci 112, 374-84 (2009).

22. Ambros, V. The functions of animal microRNAs. Nature 431, 350 - 355 (2004).

23. Bartel, D. MicroRNAs: genomics, biogenesis, mechanism, and function. Cell 116, 281 - 297 (2004).

24. Calin, G. \& Croce, C. MicroRNA signatures in human cancers. Nat Rev Cancer 6, 857 - 866 (2006).

25. Esquela-Kerscher, A. \& Slack, F. Oncomirs - microRNAs with a role in cancer. Nat Rev Cancer 6, 259 - 269 (2006).

26. Giordano, S. \& Columbano, A. MicroRNAs: new tools for diagnosis, prognosis, and therapy in hepatocellular carcinoma? Hepatology 57, 840-7 (2013). 
27. Sun, J., Lu, H., Wang, X. \& Jin, H. MicroRNAs in hepatocellular carcinoma: regulation, function, and clinical implications. ScientificW orldJournal 2013, 924206 (2013).

28. Goodman, J.I. et al. What do we need to know prior to thinking about incorporating an epigenetic evaluation into safety assessments? Toxicol Sci 116, 375-81 (2010).

29. Mirbahai, L. \& Chipman, J.K. Epigenetic memory of environmental organisms: a reflection of lifetime stressor exposures. Mutat Res Genet Toxicol Environ Mutagen 764-765, 10-7 (2014).

30. Izzotti, A. \& Pulliero, A. The effects of environmental chemical carcinogens on the microRNA machinery. International Journal of Hygiene and Environmental Health 217, 601-627 (2014).

31. Rieswijk, L. et al. Evaluating microRNA profiles reveals discriminative responses following genotoxic or non-genotoxic carcinogen exposure in primary mouse hepatocytes. Mutagenesis (2015).

32. Koufaris, C., Wright, J., Currie, R.A. \& Gooderham, N.J. Hepatic microRNA profiles offer predictive and mechanistic insights after exposure to genotoxic and epigenetic hepatocarcinogens. Toxicol Sci 128, 532-43 (2012).

33. Mitchell, A.D. et al. The L5178Y/tk+/- mouse lymphoma specific gene and chromosomal mutation assay a phase III report of the U.S. Environmental Protection Agency Gene-Tox Program. Mutat Res 394, 177-303 (1997).

34. Corvi, R. et al. ECVAM retrospective validation of in vitro micronucleus test (MNT). Mutagenesis 23, 271-83 (2008).

35. Matsushima, T. et al. Validation study of the in vitro micronucleus test in a Chinese hamster lung cell line (CHL/IU). Mutagenesis 14, 569-80 (1999).

36. Khynriam, D. \& Prasad, S.B. Cisplatin-induced genotoxic effects and endogenous glutathione levels in mice bearing ascites Dalton's lymphoma. Mutat Res 526, 9-18 (2003).

37. Valentin-Severin, I., Le Hegarat, L., Lhuguenot, J.C., Le Bon, A.M. \& Chagnon, M.C. Use of HepG2 cell line for direct or indirect mutagens screening: comparative investigation between comet and micronucleus assays. Mutat Res 536, 79-90 (2003).

38. Miller, B. et al. Evaluation of the in vitro micronucleus test as an alternative to the in vitro chromosomal aberration assay: position of the GUM Working Group on the in vitro micronucleus test. Gesellschaft fur Umwelt-Mutations-forschung. Mutat Res 410, 81-116 (1998).

39. Cahill, P.A. et al. The GreenScreen genotoxicity assay: a screening validation programme. Mutagenesis 19, 105-19 (2004).

40. Kirkland, D. \& Speit, G. Evaluation of the ability of a battery of three in vitro genotoxicity tests to discriminate rodent carcinogens and non-carcinogens III. Appropriate follow-up testing in vivo. Mutat Res 654, 114-32 (2008).

41. Brambilla, G. \& Martelli, A. Genotoxicity and carcinogenicity studies of analgesics, anti-inflammatory drugs and antipyretics. Pharmacol Res 60, 1-17 (2009).

42. Snyder, R.D. \& Green, J.W. A review of the genotoxicity of marketed pharmaceuticals. Mutat Res 488, 15169 (2001).

43. Westerink, W.M. et al. Evaluation of the Vitotox and RadarScreen assays for the rapid assessment of genotoxicity in the early research phase of drug development. Mutat Res 676, 113-30 (2009).

44. Brambilla, G. \& Martelli, A. Update on genotoxicity and carcinogenicity testing of 472 marketed pharmaceuticals. Mutat Res 681, 209-29 (2009).

45. Cao, J. et al. Curcumin-induced genotoxicity and antigenotoxicity in HepG2 cells. Toxicon 49, 1219-22 (2007).

46. Harvey, J.S., Howe, J.R., Lynch, A.M. \& Rees, R.W. The results of five coded compounds: genistein, metaproterenol, rotenone, p-anisidine and resorcinol tested in the $\mathrm{pH} 6.7$ Syrian hamster embryo cell morphological transformation assay. Mutagenesis 20, 51-6 (2005).

47. Shelby, M.D. \& Witt, K.L. Comparison of results from mouse bone marrow chromosome aberration and micronucleus tests. Environ Mol Mutagen 25, 302-13 (1995).

48. Blakey, D., Galloway, S.M., Kirkland, D.J. \& MacGregor, J.T. Regulatory aspects of genotoxicity testing: from hazard identification to risk assessment. Mutat Res 657, 84-90 (2008).

49. Wahnschaffe, U., Bitsch, A., Kielhorn, J. \& Mangelsdorf, I. Mutagenicity testing with transgenic mice. Part I: Comparison with the mouse bone marrow micronucleus test. J Carcinog 4, 3 (2005).

50. Lambert, I.B., Singer, T.M., Boucher, S.E. \& Douglas, G.R. Detailed review of transgenic rodent mutation assays. Mutat Res 590, 1-280 (2005).

51. Davis, A. et al. Evaluation of the genetic and embryotoxic effects of bis(tri-n-butyltin)oxide (TBTO), a broad-spectrum pesticide, in multiple in vivo and in vitro short-term tests. Mutat Res 188, 65-95 (1987).

52. Tsamou, M. et al. Performance of in vitro gammaH2AX assay in HepG2 cells to predict in vivo genotoxicity. Mutagenesis 27, 645-52 (2012).

53. U.S. National Library of Medicine. Toxnet Toxicological Data Network (CCRIS,HSDB, ChemIDplus and Genetox). http://toxnet.nlm.nih.gov/, (accessed on November 14, 2014) (2014). 
54. Reddy, J.K., Rao, M.S., Azarnoff, D.L. \& Sell, S. Mitogenic and carcinogenic effects of a hypolipidemic peroxisome proliferator, [4-chloro-6-(2,3-xylidino)-2-pyrimidinylthio]acetic acid (Wy-14, 643), in rat and mouse liver. Cancer Res 39, 152-61 (1979).

55. Mathijs, K. et al. Assessing the metabolic competence of sandwich-cultured mouse primary hepatocytes. Drug Metab Dispos 37, 1305-11 (2009).

56. Mathijs, K. et al. Gene expression profiling in primary mouse hepatocytes discriminates true from falsepositive genotoxic compounds. Mutagenesis 25, 561-8 (2010).

57. Rieswijk, L., Lizarraga, D., Brauers, K.J., Kleinjans, J.C. \& van Delft, J.H. Characterisation of cisplatininduced transcriptomics responses in primary mouse hepatocytes, HepG2 cells and mouse embryonic stem cells shows conservation of regulating transcription factor networks. Mutagenesis 29, 17-26 (2014).

58. Mosmann, T. Rapid colorimetric assay for cellular growth and survival: application to proliferation and cytotoxicity assays. J Immunol Methods 65, 55-63 (1983).

59. Griffiths-Jones, S., Saini, H.K., van Dongen, S. \& Enright, A.J. miRBase: tools for microRNA genomics. Nucleic Acids Research 36, D154-8 (2008).

60. Edgar, R., Domrachev, M. \& Lash, A.E. Gene Expression Omnibus: NCBI gene expression and hybridization array data repository. Nucleic Acids Res 30, 207-10 (2002).

61. Eijssen, L.M. et al. User-friendly solutions for microarray quality control and pre-processing on ArrayAnalysis.org. Nucleic Acids Res 41, W71-6 (2013).

62. Chen, J., Hsueh, H.-M., Delongchamp, R., Lin, C.-J. \& Tsai, C.-A. Reproducibility of microarray data: a further analysis of microarray quality control (MAQC) data. BMC Bioinformatics 8, 412 (2007).

63. Bourgon, R., Gentleman, R. \& Huber, W. Independent filtering increases detection power for highthroughput experiments. Proceedings of the National Academy of Sciences 107, 9546-9551 (2010).

64. Reich, M. et al. GenePattern 2.0. Nat Genet 38, 500-1 (2006).

65. Rebhan, M., Chalifa-Caspi, V., Prilusky, J. \& Lancet, D. GeneCards: integrating information about genes, proteins and diseases. Trends Genet 13, 163 (1997).

66. Ishii, H. et al. Fhit modulates the DNA damage checkpoint response. Cancer Res 66, 11287-92 (2006).

67. Okumura, H. et al. Fragile gene product, Fhit, in oxidative and replicative stress responses. Cancer Sci 100, 1145-50 (2009).

68. Karras, J.R., Paisie, C.A. \& Huebner, K. Replicative Stress and the FHIT Gene: Roles in Tumor Suppression, Genome Stability and Prevention of Carcinogenesis. Cancers (Basel) 6, 1208-19 (2014).

69. Cisternas, F.A., Vincent, J.B., Scherer, S.W. \& Ray, P.N. Cloning and characterization of human CADPS and CADPS2, new members of the Ca2+-dependent activator for secretion protein family. Genomics 81, 279-91 (2003).

70. Uehara, T. et al. A toxicogenomics approach for early assessment of potential non-genotoxic hepatocarcinogenicity of chemicals in rats. Toxicology 250, 15-26 (2008).

71. Momand, J., Wu, H.H. \& Dasgupta, G. MDM2--master regulator of the p53 tumor suppressor protein. Gene 242, 15-29 (2000).

72. Gonzalez, F.J. The peroxisome proliferator-activated receptor alpha (PPARalpha): role in hepatocarcinogenesis. Mol Cell Endocrinol 193, 71-9 (2002).

73. Szalowska, E., Tesfay, H.A., van Hijum, S.A. \& Kersten, S. Transcriptomic signatures of peroxisome proliferator-activated receptor alpha (PPARalpha) in different mouse liver models identify novel aspects of its biology. BMC Genomics 15, 1106 (2014).

74. Tamura, K., Ono, A., Miyagishima, T., Nagao, T. \& Urushidani, T. Profiling of gene expression in rat liver and rat primary cultured hepatocytes treated with peroxisome proliferators. J Toxicol Sci 31, 471-90 (2006).

75. Sokol, J.P. \& Schiemann, W.P. Cystatin C antagonizes transforming growth factor beta signaling in normal and cancer cells. Mol Cancer Res 2, 183-95 (2004).

76. Sempere, L., Sokol, N., Dubrovsky, E., Berger, E. \& Ambros, V. Temporal regulation of microRNA expression in Drosophila melanogaster mediated by hormonal signals and broad-Complex gene activity. Dev Biol 259, 9 - 18 (2003).

77. Jones, B., Roberts, P.J., Faubion, W.A., Kominami, E. \& Gores, G.J. Cystatin A expression reduces bile salt-induced apoptosis in a rat hepatoma cell line. Am J Physiol 275, G723-30 (1998).

78. Kos, J., Stabuc, B., Cimerman, N. \& Brunner, N. Serum cystatin C, a new marker of glomerular filtration rate, is increased during malignant progression. Clin Chem 44, 2556-7 (1998).

79. Agola, J.O., Jim, P.A., Ward, H.H., Basuray, S. \& Wandinger-Ness, A. Rab GTPases as regulators of endocytosis, targets of disease and therapeutic opportunities. Clin Genet 80, 305-18 (2011).

80. Sui, Y., Zheng, X. \& Zhao, D. Rab31 promoted hepatocellular carcinoma (HCC) progression via inhibition of cell apoptosis induced by PI3K/AKT/Bcl-2/BAX pathway. Tumour Biol (2015).

81. Nakayama, K.I. \& Nakayama, K. Regulation of the cell cycle by SCF-type ubiquitin ligases. Semin Cell Dev Biol 16, 323-33 (2005).

82. Wang, Z., Liu, P., Inuzuka, H. \& Wei, W. Roles of F-box proteins in cancer. Nat Rev Cancer 14, 233-247 (2014). 


\section{CHAPTER 4}

83. Tian, X. et al. F-box protein FBXO22 mediates polyubiquitination and degradation of KLF4 to promote hepatocellular carcinoma progression, (2015). 
0

O

$\bigcirc$

O

10

() 0

Chapter 5

○

Aflatoxin B1 exposure ind exes epigenetic () mechanisms in primary humato hepatocytes revealing novel biologicalprocesses associated with hepatocellular careinoma.

O

O

O

00

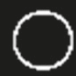

Submitted

0
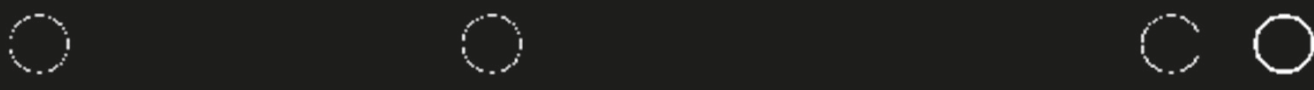

Rieswijk L.

Glaessen S.M.H. Bekers O.

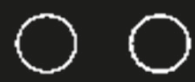

is

van Herwijnen M.

Theunissen D.H.J.

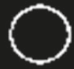

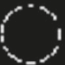



Jennen D.G.J.

de Kok M.C.M.

Kleinjans :.Q.S.

van Breda S.G.J. 


\section{$\underline{\text { Abstract }}$}

Aflatoxin B1 (AFB1) is an extremely hepatotoxic and carcinogenic mycotoxin which is, especially in third world countries, frequently reported as a food contaminant. Acute exposure to large levels of AFB1 may induce aflatoxicosis while chronic exposure is mainly associated with hepatocellular carcinoma (HCC). Recent studies suggest an AFB1-induced deregulation of epigenetic control mechanisms in HCC. In this study, we aim to identify novel biological processes contributing to AFB1-induced HCC, by in depth investigation of epigenetic mechanisms. For that, primary human hepatocytes were exposed to two doses of AFB1 for 5 days. Whole genome DNA methylation changes, also comprising microRNA promoter regions, whole genome transcriptomic analysis and microRNA expressions were analyzed applying microarray technologies, and cross-omics interactions were evaluated. Upon combining transcriptomics data with results on DNA methylation changes and microRNA expression, biological processes induced by AFB1, appeared to be linked to HCC through metabolism, nucleic acid metabolism, DNA repair, cell cycle, apoptosis, immune response and ion transport. Novel insights in association with development and cell differentiation were obtained. Specific pathways within these AFB1-induced biological processes appeared involved in signal transduction cascades. In particular, a number of microRNA-targeted genes could be identified such as ARHGAP35, ILK, PPP2R1A, RB1, TFAP2A, HIST1H4D, RHOA, CDK4 and UBC. Deregulation of these genes and signal transduction cascades represent important hallmarks of HCC. This knowledge might contribute to a better understanding of AFB1induced onset of HCC. 


\section{Introduction}

Aflatoxin B1 (AFB1), a highly hepatotoxic and carcinogenic (International Agency for Research on Cancer (IARC) class I carcinogen ${ }^{1}$ ) mycotoxin, is produced by several Aspergillus fungi strains, namely A.flavus, A. parasiticus, A.nomius and A.pseudotamarii ${ }^{2}$.

AFB1 is an indirect carcinogen that requires bioactivation by phase I cytochrome P450 (CYP) enzymes CYP1A2 and CYP3A4 to be converted into toxic intermediates, such as exo-8,9epoxide, the major and highly active epoxide metabolite of AFB1 ${ }^{3}$.

High levels of AFB1 exo-8,9-epoxides are capable of irreversibly binding to cellular macromolecules leading to persistent and highly mutagenic DNA adducts (e.g. guanine and FAPY adducts) which are extremely resistant to DNA repair. This may lead to hepatocellular carcinoma (HCC). Notably, AFB1 is known to induce a transversion mutation $(G \rightarrow T)$ in codon 249 of the p53 tumor suppressor gene ${ }^{4}$ which may silence the gene and permits uncontrolled cell proliferation. AFB1 detoxification by glutathione S-transferase may also lead to depletion of glutathione, thus resulting in high levels of reactive oxygen species (ROS) which might affect also other processes such as protein synthesis, the glycolytic pathway, lipid peroxidation and electron transport chain involved in ATP production 5. Consequently, cytotoxicity ${ }^{6}$, cellular death by apoptosis (preferential binding for mitochondrial DNA), and necrosis ${ }^{7}$ have been reported to be induced by AFB1.

Although the relation between AFB1 exposure and the development of HCC has been clearly defined in a high number of studies ${ }^{8,9}$, the potential role of epigenetic control mechanisms is less clear. Analyses of liver tissue from HCC patients in association with risk factors such as AFB1 exposure, indicate gene-specific hyper methylation (for example of RASSF1A, p16 ${ }^{10}$ and MGMT ) as well as genome-wide hypo methylation ${ }^{11}$. This appears often accompanied by a mutation in the p53 gene 4,12,13. It is suggested that AFB1 preferentially binds to methylated CpG sites and/or specific structures in chromatin, thus inducing damage to DNA and histones that may impact on methylation (causing genome-wide hypo methylation) ${ }^{14}$. Also, DNA damage generated by ROS produced by AFB1, may reduce the binding affinity of methyl-CpG binding protein 2 (MECP2), thus resulting in epigenetic alterations ${ }^{15}$.

In addition, AFB1 treatment may also influence the promotor methylation site and the expression of important microRNAs which control the expression of more than $60 \%$ of the human protein coding genes ${ }^{16}$. Depending on their particular mRNA targets, microRNAs may function as tumor suppressors or oncogenes and are frequently observed to be deregulated in HCC. An example of a microRNA that has a strong association with HCC is microRNA-221 (tumor-promoting activity ${ }^{17}$ ).

Several microRNAs have already been associated with HCC development in relation to AFB1 exposure ${ }^{18}$, for example microRNA-148 (gene targets: PXR and CYP3A4 ${ }^{19}$ ) and microRNA192 (involved in NER repair pathway genes ${ }^{20}$ ).

However, neither in HCC patient studies in relation to AFB1 exposure, nor in experimental studies on AFB1 toxicity, attempts were made to integrate the epigenome with the transcriptome in combination with affected microRNAs, in order to improve (through such a cross-omics approach) molecular understanding of carcinogenesis.

In this present study, we hypothesize that by following an integrative cross-omics approach, we will be able to identify novel methyl DNA-mRNA-microRNA interaction networks that are associated with AFB1-induced development of HCC. Therefore, primary human hepatocytes were exposed to two doses of AFB1 for 5 days. Whole genome DNA methylation changes, 
including microRNA promoter regions, were measured by NimbleGen 2.1 deluxe promoter arrays. In addition, whole genome transcriptomic analysis was performed using Affymetrix whole genome gene expression microarrays in combination with microRNA analyses using Agilent microRNA microarrays. Interaction networks were created using the biological process and pathway analysis tool within ConsensusPathDB ${ }^{21}$. Differentially methylated genes and/or transcripts, which were target of a differentially expressed microRNA, were investigated in more detail and linked with important hallmarks of cancer.

\section{Materials and methods}

\section{Cell culture, dose determination and aflatoxin B1 treatment}

Cryopreserved primary human hepatocytes $(\mathrm{PHH})$ were purchased from Life Technologies. Cells were cultured in pre-coated 24-well plates $(700,000$ cells $/ \mathrm{ml})$ in a 2-layer collagen sandwich (A11428-02, Gibco), according to the supplier's protocol (Invitrogen, 2012). The following culture media were used: Hepatocyte Thawing Medium (HTM) for thawing (CM7500, Gibco), Williams' Medium E (1x, no phenol red) (A1217601, Gibco) + Cell Maintenance supplement B kit (CM4000, Gibco) for plating and incubation. After quick thawing in a water bath at $37^{\circ} \mathrm{C}$, viability of the cells was checked by a Trypan blue (CAS no. 72-57-1, Sigma-Aldrich) exclusion test as instructed in the supplier's protocol (Invitrogen, 2012). All viability scores after thawing were in agreement with those listed by the supplier. Before AFB1 treatment, cells were allowed to acclimatize for 48 hours. This is needed for the hepatocytes to restore an in vivo like cellular configuration and enzyme expression levels as optimally as possible. AFB1 doses causing minimal (IC20) and moderate (IC40) cytotoxicity after 5 days of repetitive daily exposure were established by means of the MTT assay ${ }^{22}$. Furthermore, crucial liver function enzymes such as lactate dehydrogenase (LDH) and alanine transaminase (ALT) were measured. LDH and ALT were spectrophotometrically determined on a Cobas 8000 Modular Analyser (Roche Diagnostics, Basel, Switzerland). Based on this data, two doses, $0.3 \mu \mathrm{M}$ and $1 \mu \mathrm{M}$ of AFB1 (CAS no. 1162-65-8, Sigma-Aldrich), were selected for the main experiment. Control cells were exposed to $0.5 \%$ of the vehicle solvent dimethyl sulfoxide (DMSO, CAS no. 67-68-5, Sigma-Aldrich).

To diminish the possible impact of interdonor variability, PHH from three donors of different sex, ethnicity, age, body mass index and behavioral status concerning smoking, alcohol and drug use (see Supplementary Table 5.1) were pooled. Cells were cultured in triplicate in 24 wells (RNA) or 6 wells (DNA) in a collagen sandwich layer. Following 5 days of repetitive daily exposure, cells lysates were harvested for DNA and total RNA isolation.

\section{DNA methylation analyses}

\section{DNA isolation}

Cells were lysed in $500 \mu \mathrm{l}$ digestion buffer (containing 0.5 M EDTA; $1 \mathrm{M}$ Tris-HCl, pH 8.0; $10 \%$ SDS ) and incubated for 1 hour at $55^{\circ} \mathrm{C}$. Next, $25 \mu$ l of proteinase $\mathrm{K}(20 \mathrm{mg} / \mathrm{ml})$ (Ambion) was added. After incubation of 1 hour at $55{ }^{\circ} \mathrm{C}$, the proteinase $\mathrm{K}$ was inactivated for 10 minutes at $80{ }^{\circ} \mathrm{C}$. RNAse A $(2 \mu \mathrm{l} ; 100 \mathrm{mg} / \mathrm{ml})$ (Qiagen) and collagenase $(25 \mu \mathrm{l} ; 1 \%)$ (Sigma) treatment was performed for 1 hour at $37{ }^{\circ} \mathrm{C}$. Thereupon, $500 \mu \mathrm{l}$ of phenol-chloroformisoamylalcohol (PCI; 25:24:1) (Sigma) was added. The mixture was shaken manually for 5 min, and centrifuged for 5 minutes at maximum speed. The upper phase was transferred to a new Eppendorf and the step with PCI was repeated. The upper phase was collected and precipitated using $50 \mu \mathrm{l} 3 \mathrm{M} \mathrm{NaAc} \mathrm{pH} 5.6$ and $1250 \mu \mathrm{l}$ cold $100 \%$ ethanol for $30 \mathrm{~min}$. at $-80^{\circ} \mathrm{C}$. After centrifugation for 30 minutes at maximum speed, the DNA pellet was washed using cold 
$70 \% \mathrm{ETOH}$, dried in a speed vac and dissolved in $50 \mu \mathrm{l}$ nuclease free water. The total amount was at least $10 \mu \mathrm{g}$ DNA, the 260/280 ratio ranged between 1.7-1.9, and the 260/230 ratio appeared higher than 1.6. A total of 9 DNA samples were prepared.

Methylated DNA Immunoprecipitation (MeDIP), whole genome amplification (WGA) and methylation enrichment assessment

In order to obtain fragments ranging from $200 \mathrm{bp}$ to $600 \mathrm{bp}$, genomic DNA was sonicated. Next, the fragments were cleaned up using silica columns (Zymo Research) and eluted in TE buffer.

MeDIP was performed using the MagMeDIP kit (Diagenode, Liege, Belgium) according to the manufacturer's protocol. Briefly, IP incubation mix was added to $1.2 \mu \mathrm{g}$ sonicated DNA sample and denatured at $95^{\circ} \mathrm{C} .10 \%$ of this was used as reference samples. The remaining sample was immunoprecipitated overnight using antibody mix containing the 5-methylcytidine antibody and magnetic beads. Following purification using the Ipure kit (Diagenode), reference and MeDIP samples were prepared for microarray analysis by whole genome amplification (WGA) using the WGA2 kit (Sigma-Aldrich) without performing the fragmentation step. Methylation enrichment in the paired samples MeDIP/reference was derived from qPCR data by calculating the ratio positive control/negative control, applying the $\Delta \Delta \mathrm{Ct}$ method.

\section{MeDIP-Chip}

The Human DNA Methylation 2.1M Deluxe Promoter Array (Roche NimbleGen) was used for analyses of DNA methylation levels. Labeling and hybridization of arrays were performed according to the manufactures' protocol. Slides were washed using the NimbleGen wash buffer kit and scanned using the $2 \mu \mathrm{m}$ high resolution NimbleGen MS 200 microarray scanner.

DNA methylation data analyses and selection of differentially methylated genes (DMGs)

Signal intensity data was extracted from the scanned images of each array using NimbleScan v2.6 software and quantile normalized on a per channel basis. Log2 ratios of the intensities were computed (ratio of MeDIP signal / Input signal) and for each array, centering was performed by subtracting the global array bi-weight mean of the $\log 2$ ratios such that the computed $\log 2$ ratios were centered around 0 .

Detection of differential methylation was performed using the Probe Sliding WindowANOVA algorithm (PSW-ANOVA). PSW-ANOVA was implemented in the $\mathrm{R}$ statistical programming environment (v2.15.3) (http://www.r-project.org) as a custom script and was provided by Roche NimbleGen as described before ${ }^{23}$.

PSW-ANOVA (sliding window of 750 bp comprising 7 probes, and a FDR corrected p- value $<0.01$ ) was used to identify differential methylated regions (DMR) which were statistically significantly different between the different conditions tested in the experiment i.e. Exposed versus Control. Peaks were identified in the DMR by searching for regions containing at least 8 significant consecutive probes $(\mathrm{p}<0.01)$.

Peaks were mapped to promoter regions (from $3 \mathrm{~kb}$ upstream to $1 \mathrm{~kb}$ downstream of the transcription start site ${ }^{24}$ ) and $\mathrm{CpG}$ islands of genes using the NimbleScan v2.6 software. A control corrected median $\log 2$ ratio was calculated for each peak. Log2 ratio's $>0$ indicate hyper methylation and $\log 2$ ratio's $<0$ indicate hypo methylation.

The DNA methylation microarray data have been deposited in NCBI's Gene Expression Omnibus (GEO) ${ }^{25}$ and are accessible through accession number GSE67005. 
MeDIP qPCR: validation of DNA methylation array

Standard quantitative PCR analyses were carried out for 7 genes (GLCE, TRAM1, DKK1, GNG5, LNX1, DHRS7 and ENOPH1) using $5 \mathrm{ng}$ of MeDIP and reference samples on a Bio$\mathrm{Rad} \mathrm{MyiQ}^{\mathrm{TM}}$. A list of primers is available in Supplementary Table 5.2. The results of the validation procedure were presented in Supplementary Figure 5.1.

\section{Gene expression analyses}

\section{Total RNA isolation and quality control}

At the end of treatment, medium was removed and PHH were harvested in Qiazol (Qiagen). Total RNA was isolated using a miRNeasy Mini Kit (Qiagen) according to the manufacturer's protocol and followed by DNase I (Qiagen) treatment. Upon purification, RNA concentrations were measured by means of a NanoDrop ${ }^{\circledR}$ ND-1000 spectrophotometer (Thermo Scientific) at 260 and $280 \mathrm{~nm}$. RNA quality and integrity were assessed by using automated gel electrophoresis on an Agilent 2100 Bioanalyzer system (Agilent Technologies). Only RNA samples which showed clear $18 \mathrm{~S}$ and $28 \mathrm{~S}$ peaks and with an RNA integrity number (RIN) higher than 8 , were used. Samples were stored at $-80^{\circ} \mathrm{C}$ until RNA hybridization.

\section{Labelling, hybridization, re-annotation}

High-density oligonucleotide GeneChips from Affymetrix were used to measure gene expression levels (Human Genome U133 Plus 2.0 array (604,258 probes)). Targets for these arrays were prepared, hybridized and scanned according to the Affymetrix protocol (Affymetrix).Normalization quality controls appeared to be within acceptable limits for almost all chips.

The mRNA microarray data have been deposited in NCBI's Gene Expression Omnibus (GEO) ${ }^{25}$ and are accessible through accession number GSE67002.

\section{Re-annotation, normalization and data filtering}

The Arrayanalysis.org web service was used for quality control ${ }^{26}$ and all microarrays except one were of high quality which was omitted from the analyses. CEL files were imported into R v2.15.3 (http://www.r-project.org) using the "affy" library 27 within BioConductor (v2.9) 28. Probe re-annotation, normalization and data filtering was performed as previously described ${ }^{29}$.

\section{$\underline{\text { Selection of differentially expressed genes (DEGs) }}$}

Differentially expressed genes (DEGs) were selected using the linear model for microarrays (LIMMA) approach ${ }^{30}$. The resulting p-values were FWER-corrected using the False Discovery Rate (FDR) method. The following criteria were applied: (1) 1) average expression in at least one of the experimental groups $>6$ (log2-scale), (2) a FDR-corrected p-value $<0.05$ obtained through a moderated t-test, and (3) for the three replicates an average absolute fold change (FC) of 1.5 or higher (i.e., average $\log 2$ ratio of $<-0.58$ or $>0.58$ ).

\section{MicroRNA expression analyses}

Hybridization, normalization and selection of differentially expressed microRNAs (DE-miRs)

MicroRNA expression profiling was performed using Agilent Sureprint G3 Unrestricted Human microRNA V19 $8 \times 60 \mathrm{~K}$ microarrays. The hybridization was performed following standard protocols, after which the microarray slides were washed and scanned using a DNA microarray scanner (Agilent Technologies). The scanned images were converted into TXT files 
using the Feature Extraction Software v10.7.3.1 from Agilent Technologies, which were imported in R 2.15.3 (http://www.r-project.org) for quality control with an in-house developed pipeline. Filtering and normalization was performed using AgiMicroRna ${ }^{31}$. Total gene signals were $\log 2$-transformed and quantile-normalized. The selection criteria for determining differentially expressed microRNAs (DE-miRs) were identical to those for DEGs.

The microRNA microarray data, have been deposited in NCBI's Gene Expression Omnibus (GEO) ${ }^{25}$ and are accessible through accession number GSE67003.

\section{Target information of DE-miRs}

To understand the biological function of DE-miRs, target information was retrieved using a database containing over 50,000 experimentally validated microRNA-mRNA interactions (miRTarBase - Homo sapiens, release 4.5, miRBase version 20). MiRTarBase 32 (http://mirtarbase.mbc.nctu.edu.tw/). Additionally, predicted mRNA targets were considered of interest. For this purpose, miRSystem ${ }^{33}$ (http://mirsystem.cgm.ntu.edu.tw/) was used; this database integrates seven well-known microRNA target gene prediction algorithms: DIANA, miRanda, miRBridge, PicTar, PITA, rna22, and TargetScan. This database also contains validated data from TarBase and miRecords on the interaction between a microRNA and its target genes. Only microRNA-mRNA interactions that were predicted by 3 or more prediction algorithms were taken into account.

\section{Biological interpretation of DEMs, DEGs and DE-miRs}

A step-by-step approach (depicted in Figure 5.1) was followed in order to visualize a priori known as well as newly associated AFB1-induced methylated and differentially expressed genes, microRNAs and biological processes in relation to HCC in an integrative interaction network using Cytoscape. Biological process (GO) and pathway analysis ( $\mathrm{p}$-value $<0.01,>2$ genes), using ConsensusPathDB ${ }^{21}$, was performed for biological interpretation of obtained 'omics' data. In step 1 the lists of DEGs and DMGs obtained following exposure to a low or moderate dose of AFB1 were compared with the list of a priori known AFB1-induced genes (obtained by data curated in the Comparative Toxicogenomics Database (CTD) (http://ctdbase.org/) ${ }^{34}$ ) and to a HCC signature gene list (created by Caiment et al. (2014) ${ }^{35}$ by using publically available gene expression data sets from ArrayExpress (HCC versus healthy human liver)). The Venn diagram in step 1 of Figure 5.1 displays all the newly AFB1-induced DEGs, DMGs and their overlap, which play a role in HCC. The list of 2823 genes was named list A (see part A of Supplementary Table 5.4). In step 2 list B (see part B of Supplementary Table 5.4) was created existing of 1781 a priori known AFB1-induced genes which play a role in HCC (see Venn diagram step 2 of Figure 5.1). In step 3 list A and B were used separately for biological (GO) process analysis within ConsensusPathDB. For visualization, the biological processes were categorized into more generic biological terms. In step 4 all significantly DEGs and DMGs that were involved in the obtained biological processes using list $\mathrm{A}$ and $\mathrm{B}$ were extracted. This list of 2945 genes was then uploaded into Cytoscape and by using the MiMI plugin (with the option for "Interactions among query genes (include at least 2 genes)" 36 )-and Cy'TargetLinker (based on the regulatory interaction network (RIN) of miRTarBase v.4.5 ${ }^{37}$ ) plugin, gene-gene and validated microRNA-gene interactions respectively were obtained in step 5. For 1431 out of 2945 genes at least one gene-gene interactions were identified. In step 6 the list of 1431 genes was then visualized together with the microRNA-gene interactions of 6 microRNAs in Cytoscape. The MultiColoredNodes plugin ${ }^{38}$ was used for visualization of the expression values (methylation as well as the gene expression level) following a low or a moderate dose of AFB1. 


\section{$\underline{\text { Results }}$}

\section{Identification of differentially expressed genes (DEGs)}

Exposure of a pool of PHH to a low $(0.3 \mu \mathrm{M})$ and moderate dose $(1 \mu \mathrm{M})$ of AFB1 resulted in statistically significant changes in gene expressions and in the methylation status. Exposure to a low or a moderate dose of AFB1 induced an overlapping 1430 DEG (749 down regulated genes and 681 upregulated genes) (see Venn diagram in part A of Figure 5.2). The low AFB1 dose induced an additional set of 60 uniquely modulated DEGs (40 down regulated genes and 20 upregulated genes) while the higher dose modified the expression of 1125 genes (408 down regulated genes and 717 upregulated genes) (see part A of Supplementary Table 5.3 for the expression values).

\section{Step-by-step approach for integrative data analysis}

To identify novel processes by which AFB1 can initiate/promote HCC

Step 1: Creation of list A) Identification of novel genes which respond to AFB1 and play a role in $\mathrm{HCC}$
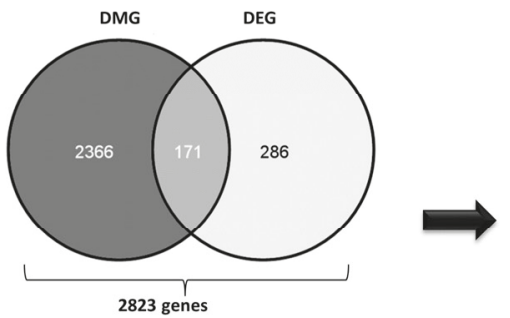

Step 2: Creation of list B) Identification of genes previously known to respond to AFB1 and play a role in HCC:
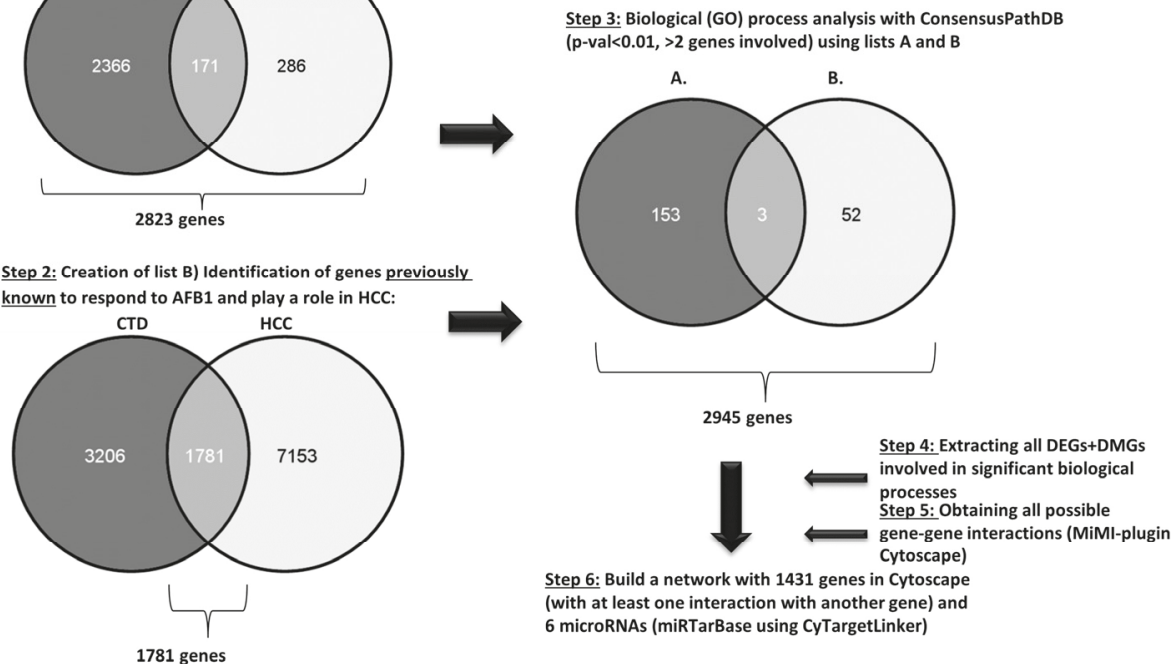

1781 genes

Figure 5.1: A step-by-step approach was followed in order to visualize a priori known and newly associated AFB1induced methylated and differentially expressed genes, microRNAs and biological processes in relation to HCC in an integrative interaction network using Cytoscape. 
A.

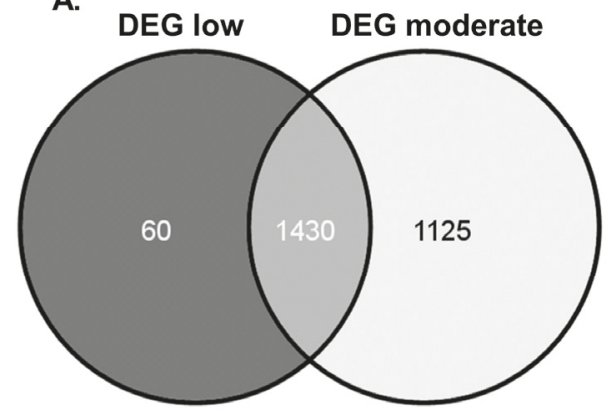

B.

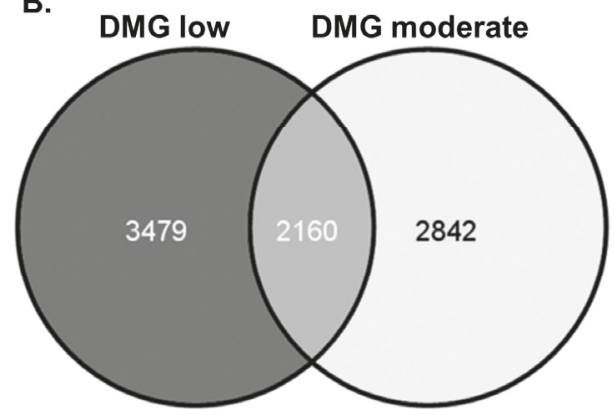

C.

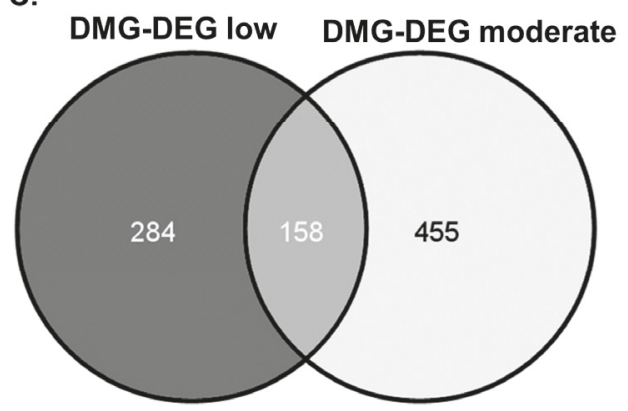

Figure 5.2: Identification of common and unique effects of 5 days repetitive exposure to a low $(0.3 \mu \mathrm{M})$ or a moderate $(1 \mu \mathrm{M})$ dose of aflatoxin B1 on (A) differentially expressed genes (DEG), (B) differentially methylated genes (DMG) and (C) differentially expressed genes with a significant change in methylation status (DMG-DEG) in a pool of primary human hepatocytes $(\mathrm{AFB} 1=$ aflatoxin $\mathrm{B} 1$, low $=$ low dose, moderate $=$ moderate dose $)$

\section{Identification of differentially methylated genes (DMGs)}

Exposure at these doses of AFB1 resulted in 2160 overlapping differentially methylated genes (see Venn diagram in part B of Figure 5.2). For these 2160 genes, the direction of change was the same for both doses (1297 hypo methylated genes and 863 hyper methylated genes). An additional 376 genes showed in both doses a change in methylation status in different directions (low dose: 293 hypo methylated genes and 83 hyper methylated genes; moderate dose: 83 hypo methylated genes and 293 hyper methylated genes). In addition, 3103 (2153 hypo methylated genes and 950 hyper methylated genes) respective 2466 (1111 hypo methylated genes and 1355 hyper methylated genes) DMG showed a unique response following a low respective moderate dose of AFB1 (see part B of Supplementary Table 5.3 for the expression values).

\section{Identification of differentially expressed genes with a change in their methylation} status

In the next step, genes that showed a statistically significant change in their methylation status and their gene expression following exposure to AFB1 were identified. Among these identified genes were 158 genes affected following exposure to both doses of AFB1 (same direction of change on methylation and gene expression level) (see Venn diagram in part C of Figure 5.2). Of these 158 genes, 19 were hyper methylated and down regulated while 41 genes were hypo methylated and upregulated. An additional 29 genes showed at both doses a change in methylation status as well as on the gene expression level, however the direction of change of especially the methylation status is different (see Table 5.1 for more details). In addition, 255 
and 426 genes showed a unique statistically different response for gene expression and methylation status at the low and moderate dose respectively (see part C of Supplementary Table 5.3 for the expression values). Overall, following exposure to AFB1, relatively more genes appeared hypo methylated instead of hyper methylated, and demonstrated an up regulation in their expression.

Table 5.1: Proportion of common and unique differentially expressed genes that show a change in their methylation status in PHH following 5 days daily exposure to a low and moderate dose of aflatoxin B1 (the bold numbers represent the genes that are hypo methylated and upregulated or hyper methylated and down regulated).

\begin{tabular}{|c|c|c|c|c|}
\hline \multicolumn{2}{|r|}{ Common } & \multicolumn{2}{|c|}{ DEG } & \multirow[t]{2}{*}{ Total } \\
\hline & & Upregulated $(+)$ & Downregulated (-) & \\
\hline \multirow{3}{*}{ DMG } & Hypermethylated $(+)$ & 26 & 19 & 45 \\
\hline & Hypomethylated (-) & 41 & $\overline{72}$ & 113 \\
\hline & Total & 67 & 91 & \\
\hline
\end{tabular}

\begin{tabular}{|c|c|c|c|c|}
\hline \multicolumn{2}{|c|}{ Unique low dose } & \multicolumn{2}{c|}{ DEG } & \multirow{2}{*}{ Total } \\
\cline { 3 - 4 } \multicolumn{2}{|c|}{ DMG } & Upregulated $(+)$ & Downregulated $(-)$ & \\
\cline { 2 - 5 } & Hypomethylated $(+)$ & 32 & 28 & 60 \\
\cline { 2 - 5 } & Tothylated $(-)$ & 82 & 142 & 224 \\
\hline
\end{tabular}

\begin{tabular}{|c|c|c|c|c|}
\hline \multirow{2}{*}{\multicolumn{2}{|c|}{ Unique moderate dose }} & \multicolumn{2}{|c|}{$\overline{\text { DEG }}$} & \multirow{3}{*}{$\begin{array}{c}\text { Total } \\
233\end{array}$} \\
\hline & & $\overline{\text { Upregulated }(+)}$ & Downregulated (-) & \\
\hline \multirow{3}{*}{ DMG } & Hypermethylated $(+)$ & 158 & 75 & \\
\hline & Hypomethylated (-) & 116 & 106 & 222 \\
\hline & Total & 274 & 181 & \\
\hline
\end{tabular}

\section{Identification of differentially expressed microRNAs (DE-miRs)}

Exposure of $\mathrm{PHH}$ to AFB1 resulted in a set of 19 statistically significantly expressed microRNAs for the moderate dose (see part D of Supplementary Table 5.3 for the expression values). 4 microRNAs were down regulated (e.g. hsa-miR-211-3p) and 15 were upregulated (e.g. hsa-miR-181b-5p, hsa-miR-34b-5p, hsa-miR-221-3p and hsa-miR-222-3p (see part D of Supplementary Table 5.3 for more details). At the low dose, no statistically significant microRNAs could be detected.

\section{Methylation status of differentially expressed microRNAs}

From the list of 19 statistically significant microRNAs, following exposure to a moderate dose of AFB1, only hsa-miR-34b-5p showed a change in its methylation status (hypo methylation).

\section{mRNA target integration using miRTarBase and miRSystem}

For 7 out of the 19 identified DE-miRs, 572 targets (163 (miRTarBase) and 446 (miRSystem) of which 37 commonly identified microRNA-mRNA interactions) could be identified. For the other 12 microRNAs, no information on validated mRNA targets information is available. Targets could be found in particular for hsa-miR-130b-3p, hsa-miR-181b-5p, hsa-miR-221-3p, hsa-miR-222-3p, hsa-miR-34b-5p, hsa-miR-564 and hsa-miR-99b-5p. The complete set of predicted and validated gene targets obtained by miRSystem and miRTarBase can be found in part E and F of Supplementary Table 5.3. 


\section{Biological interpretation of differentially expressed genes (DEGs) using ConsensusPathDB}

\section{Integrated AFB1 exposure- and HCC-related response patterns}

3 resp. 150 biological processes belonging to the biological term "Cell differentiation" (312 genes) resp. "Development" (333 genes) have not earlier been identified as being associated with AFB1 exposure. The biological processes related with "Cell differentiation" were specifically involved in cell differentiation and regulation of localization. Processes grouped under "Development", appeared involved in multiple developmental processes such as cell, tissue and organ development but also transmembrane transport, morphogenesis and cell adhesion (see part C of Supplementary Table 5.4).

Biological terms in confirmation of a priori known AFB1- and HCC-related genes, were "Metabolism" (1 GO process), "Nucleic acid metabolism" (3 GO processes), "DNA repair" (3 GO processes), "Cell cycle" (37 GO processes), "Apoptosis" (2 GO processes) and "Immune response" (6 GO processes) (see part D of Supplementary Table 5.4).

Three AFB1-modified biological processes, appearing in both input gene lists, were assigned to the term of "Ion transport".

Figure 5.1 presents the workflow concerning the integration of the obtained significant biological processes within Cytoscape. 1431 genes (see part E of Supplementary Table 5.4) in total appeared involved in AFB1 exposure-related biological processes and were thus used to generate an integrated network, depicted in Figure 5.3. One remarkable finding is that all processes displayed in Figure 5.3, contain genes that were under post-transcriptional regulation of one or more of the found DE-miRs.

Specific role of novel methyl DNA-mRNA-microRNA interactions within HCC development

In order to gain more insight into the specific pathways, over-representation analyses using ConsensusPathDB were performed in parallel with the GO processes analyses. The pathways obtained using the list of genes newly observed as well as a priori known to be related to AFB1 exposure, can be found in part A and B of Supplementary Table 5.5 respectively. Only pathways were selected that contained genes that were also present within the previously generated integrated network (Figure 5.3). All newly AFB1-associated pathways appeared related with signal transduction cascades. A high number of these pathways actually describes signal transduction cascades in which $G$ protein-coupled receptors (GPCRs) are involved. Also, signal transduction cascade pathways related with retinoblastoma protein $(\mathrm{Rb})$, appeared affected by genes newly found to be induced by AFB1. These signal transduction pathways which are mainly stimulated by ligands, such as growth factors, are important in both biological process terms "Development" and "Cell differentiation" (see part C of Supplementary Table 5.5). Some examples of genes involved in these signal transduction cascades which were also under epigenetic control (presenting a change in their methylation status and/or being regulated by a microRNA) and important in "Cell differentiation", were Rho GTPase activating protein 35 (ARHGAP35), integrin-linked kinase (ILK), protein phosphatase 2, regulatory subunit A, alpha (PPP2R1A), retinoblastoma 1 (RB1), transcription factor AP-2 alpha (activating enhancer binding protein 2 alpha) (TFAP2A) and histone cluster 1, H4d (HIST1H4D). Other examples of genes involved in these signal-transduction cascades related with "Development" were ras homolog family member A (RHOA), cyclin-dependent kinase 4 (CDK4) and ubiquitin $\mathrm{C}$ (UBC). All of these genes were under post-transcriptional regulation of hsa-miR-181b-5p, hsa-miR-222-3p, hsa-miR-130b-3p, hsa-miR-221-3p or hsa-miR-34b-5p (for more details see part D and E of Supplementary Table 5.5). 


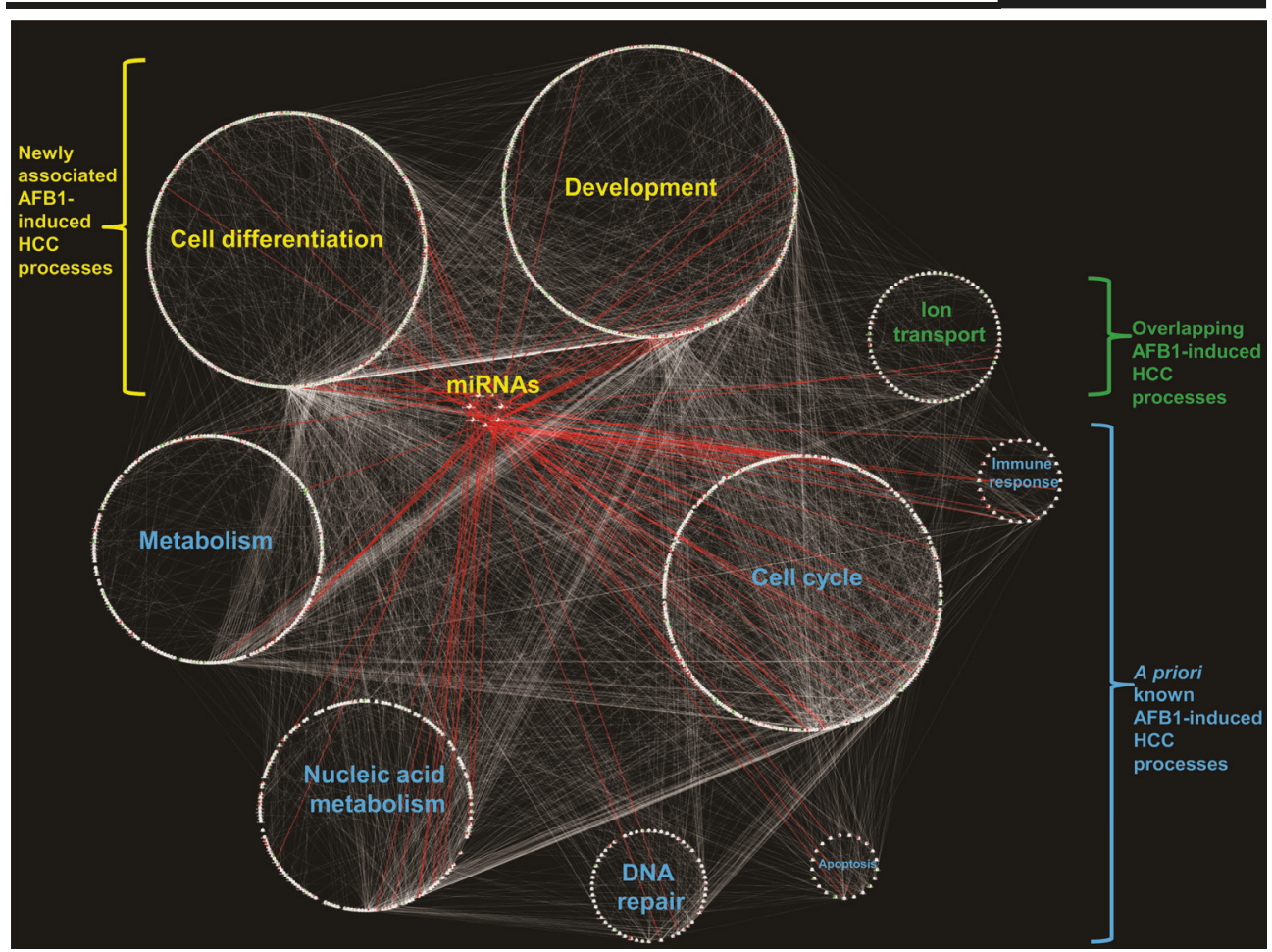

Figure 5.3: Network of 1431 a priori known and newly associated AFB1-induced genes which play a role in HCCrelated processes, as generated using a step-by-step approach depicted in Figure 5.1, together with 6 microRNAs was visualized in Cytoscape. The general biological processes "Development" and "Cell differentiation" in the upper part of the figure which are depicted in yellow represent newly associated AFB1-induced processes which play a role in HCC as found with list A (see Figure 5.1). The general processes marked in green are the overlapping processes found using the list of newly associated (A) and a priori known (B) AFB1-induced genes. The lower part of the figure, marked in blue, depicts all the general biological processes which were found using list (B) of a priori known AFB1induced processes which play a role in HCC. The finding that several genes in these general biological processes were microRNA-targets was also a new finding and therefore "microRNAs" were also depicted in yellow.

\section{$\underline{\text { Discussion }}$}

Primary human hepatocytes that were exposed to a low or a moderate dose of AFB1 for 5 days daily showed significant changes in their methylation status as well as in their gene expression level. Thousands of genes were differentially expressed by both doses and a large amount of these genes were commonly expressed. Remarkable was that, following exposure to a low dose of AFB1, more changes occur on the methylation level in comparison to the moderate dose. Upon comparing the doses with respect to their induced methylation level, again a large number of genes were demonstrated to be commonly affected. After the two data sets were integrated, thus generating lists of differentially expressed genes with a changed methylation status, a smaller number of genes persisted. It appeared that exposure to AFB1 resulted in proportionally more hypo methylated genes. This was consistent with hypo methylation levels measured in tissue from HCC patients associated with AFB1 exposure ${ }^{11}$. In addition, this study confirmed earlier reports on AFB1-induced gene-specific hyper methylation ${ }^{10}$. Overall, by studying whole genome DNA methylation changes, genes could be identified that were hypo- or hyper methylated, which had not previously been reported to be associated with 
AFB1 exposure, in addition to modifications of genes already known to be involved in AFB1induced response pathways.

MicroRNA promotors were also investigated for changes in their methylation status. Only hsamiR-34b-5p was hypo methylated by AFB1 and its expression appeared significantly increased. Transcription of hsa-miR-34b has been shown to be directly induced by p53 in response to genotoxic stress, acting upon downstream targets to promote cell cycle arrest or apoptosis ${ }^{39}$. Hsa-miR-34b-5p is furthermore able to affect hundreds of genes involved in tumor development, among for example HCC 40,41. Other microRNAs differentially expressed following AFB1 exposure and associated with HCC development before, were hsa-miR-99b ${ }^{42}$, hsa-miR-181b-5p 43, hsa-miR-221-3p 44 and hsa-miR-222-3p 45. Deregulation of these microRNAs within HCC may result in promoted growth, clonogenic survival, migration and invasion of HCC cells ${ }^{43}$. The remaining $14 \mathrm{DE}$-miRs have not been associated before with HCC development and were therefore novel findings.

Furthermore, we confirm biological processes previously associated with AFB1 exposure and also related to HCC e.g. "Metabolism", "Nucleic acid metabolism", "DNA repair", "Cell cycle", "Apoptosis", "Immune response" and "Ion transport". We observed that the low dose mainly induced changes in the processes of "Nucleic acid metabolism", "DNA repair", "Immune response" and "Ion transport" while the moderate dose mainly modified the processes of "Apoptosis" and "Cell cycle". Both doses had an effect on processes related with "Metabolism". More importantly, novel biological processes with their respective genes and microRNAs were identified that provide new insights into the role of epigenetic mechanisms in AFB1-induced HCC. The novel biological processes that have been identified were involved in "Development" and "Cell differentiation". The low dose mainly affected processes related with "Development" while the moderate dose affected processes related with "Cell differentiation".

All specific pathways that were associated with these novel biological processes concern signal transduction cascades and cell differentiation. Mainly genes affected by the low dose were involved in these important signal transduction cascades while the genes affected by the moderate dose were more involved in pathways related with cell differentiation and division. AFB1 is known to irreversible change macromolecules such as DNA, RNA and proteins thereby affecting a whole range of downstream processes related with for example growth, differentiation, and survival of a cell ${ }^{46}$. AFB1 may therefore affect a large number of signal transduction elements (e.g. activating growth factors, chemokines, ligands and receptors) which interact through complex biochemically related networks normally important in a range of developmental processes. Earlier studies have already shown that AFB1-related mutations in the p53 tumor suppressor gene (TP53) within the context of HCC may lead to the activation of the WNT signal transduction pathway ${ }^{13}$. As observed in this study, AFB1-induced changes in the signal transduction cascades may therefore affect processes such as "Apoptosis", "Cell cycle" and "Cell differentiation". AFB1-induced aberrations in signal transduction cascades may therefore lead to increased proliferative potential, sustained angiogenesis, tissue invasion and metastasis, insensitivity to growth signals and apoptosis inhibition which are characteristic hallmarks of cancer, particularly HCC ${ }^{47}$.

We were able to identify newly AFB1-affected key genes within these signal transduction cascades that were found to be targeted by deregulated microRNAs, such as integrin-linked kinase (ILK), Protein phosphatase 2, regulatory subunit A, alpha (PPP2R1A), Retinoblastoma 1 (RB1), Transcription factor AP-2 alpha (activating enhancer binding protein 2 alpha) 
(TFAP2A), Histone cluster 1, H4d (HIST1H4D), Ras homolog family member A RHOA, Cyclin-dependent kinase 4 (CDK4) and Ubiquitin C (UBC).

One interesting example of a newly identified AFB-affected methyl DNA-mRNA-microRNA interaction, obtained using an integrative cross-omics approach and involved in biological processes associated with the development of HCC, is ILK (Figure 5.4).
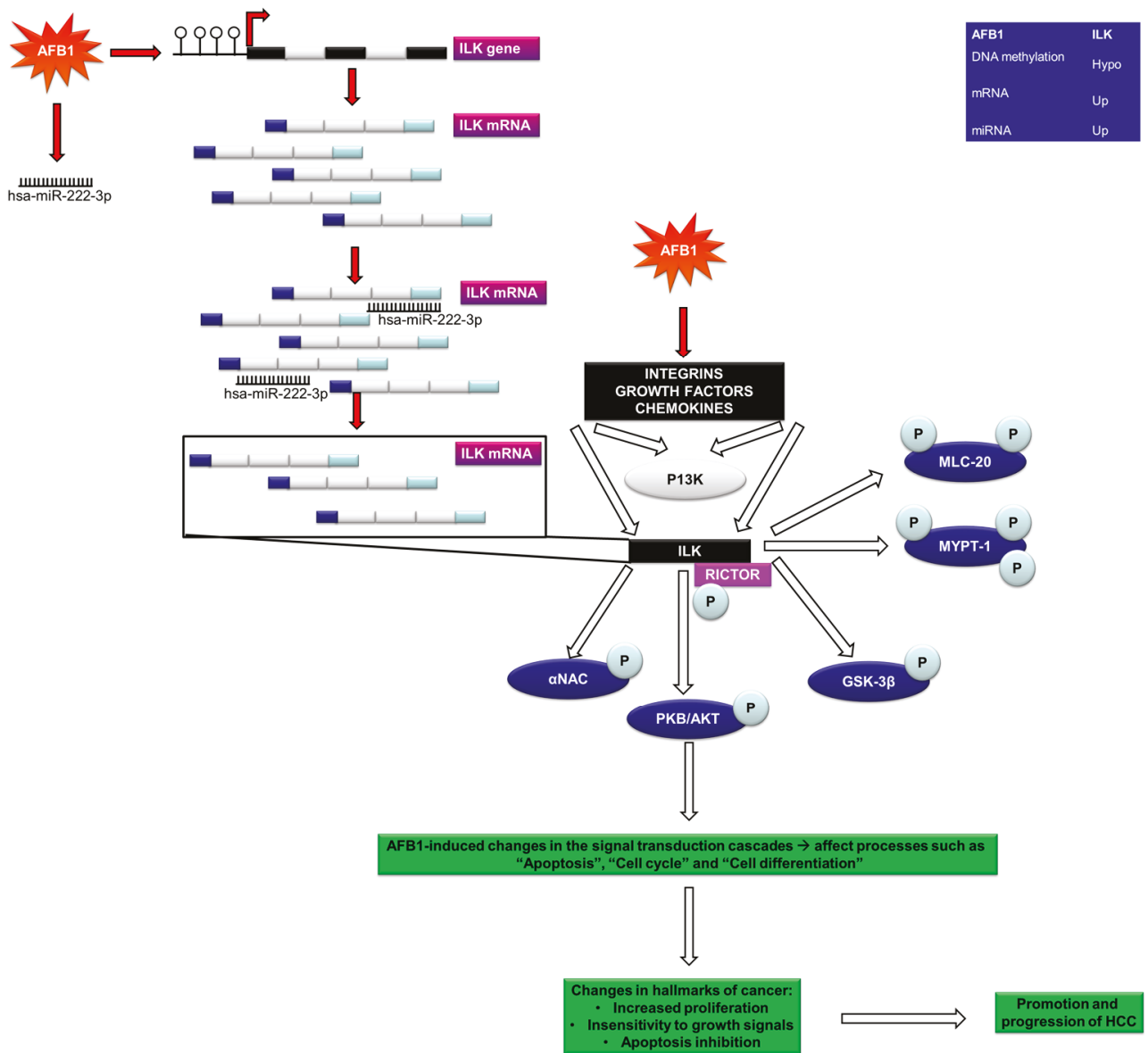

Figure 5.4: Visual representation of one interesting example of a newly identified AFB-affected methyl DNA-mRNAmicroRNA interaction; hypo methylated ILK - upregulated ILK mRNA under the control of hsa-miR-222-3p, as obtained using an integrative cross-omics approach and involved in biological processes associated with the development of HCC. In addition, the phosphorylation targets of ILK are depicted (adopted from Hannigan et., $2011^{48}$ ) which play a role in several signal transduction cascades affecting processes such as "Apoptosis", "Cell cycle" and "Cell differentiation". AFB1-induced changes of these important pathways may ultimately lead to the hallmarks of cancer characteristic for the promotion and progression of HCC $(\alpha \mathrm{NAC}=$ nascent polypeptide-associated complex and coactivator $\alpha$, GSK-3 $\beta=$ glycogen synthase kinase-3, ILK=integrin-linked kinase MYPT1= myosin phosphatase target subunit 1 and MLC20 = myosin light chain 20, PKB $/$ Akt $=$ protein kinase B, RICTOR $=$ RPTOR Independent Companion of MTOR).

For ILK, also known as integrin-linked kinase, a cross-omics response is observed on the methylation, gene expression and microRNA expression level. ILK, which appeared hypo methylated and upregulated by AFB1, is an intracellular protein, which interacts with the 
cytoplasmic domains of integrin beta and beta3 subunits. The essential role of ILK is connecting integrins to the actin cytoskeleton. ILK interacts, apart from integrins, with several adaptor and signaling proteins resulting in its activation and localization to focal adhesion plaques. Upon integrin/growth factor/chemokine binding the kinase activity of ILK is stimulated in a PI-3Kinase-dependent manner. AFB1 may potentially have an effect on the release of these stimulants (e.g. chemokines) and therefore on the function of ILK. However AFB1 may also directly affect the expression of ILK. ILK is capable of phosphorylating a variety of intracellular substrates, for example; protein kinase B (PKB/Akt), glycogen synthase kinase-3 (GSK-3), myosin light chain 20 (MLC20), nascent polypeptide-associated complex and coactivator $\alpha(\alpha \mathrm{NAC})$, myosin phosphatase target subunit 1 (MYPT1) and RPTOR Independent Companion of MTOR) (RICTOR) ${ }^{48}$. In normal cells, this type of reactions is essential for development ${ }^{49}$. AFB1-induced overexpression of ILK may result in oncogenic transformation and progression to invasive and metastatic phenotypes by activating the integrin-focal adhesion kinase (FAK)/c-Src pathway, and may also contribute to tumor growth via the AKT and glycogen synthase kinase $3 \beta$ pathways. In addition, ILK expression and activity are upregulated in several types of cancers ${ }^{50}$ and may therefore promote and progress HCC development. The expression of ILK seems to be fine-tuned by hsa-miR-222-3p here found to be differentially expressed by AFB1, which has previously been associated with HCC development ${ }^{45}$.

The regulation of gene expression is directed by a complicated interplay between DNA methylation, histone modifications, microRNAs and transcription factors. In this study, we were able to identify novel methylated genes, expressed genes and microRNAs as well as biological processes that were affected following AFB1 exposure in the context of HCC development. Alterations in these newly reported AFB1-associated biological processes, related with signal transduction cascades, may ultimately lead to changes in characteristic hallmarks of cancer such as increased proliferative potential, insensitivity to growth signals and apoptosis inhibition. In addition, this study has shown that key players of these AFB1-induced signal transduction cascades were under epigenetic and microRNA-targeted control. By applying a cross-omics integrative approach, this study is the first of its kind to identify novel genes, microRNAs and highly relevant biological processes in human target cells in vitro.

\section{Supplementary data}

Supplementary Figures 5.1 and Tables 5.1-5.5 are available upon request. 


\section{References}

1. Vainio, H., Coleman, M. \& Wilbourn, J. Carcinogenicity evaluations and ongoing studies: the IARC databases. Environ Health Perspect 96, 5-9 (1991).

2. Cary, J.W., Klich, M.A. \& Beltz, S.B. Characterization of aflatoxin-producing fungi outside of Aspergillus section Flavi. Mycologia 97, 425-32 (2005).

3. Bedard, L.L. \& Massey, T.E. Aflatoxin B1-induced DNA damage and its repair. Cancer Lett 241, 174-83 (2006).

4. Soini, Y. et al. An aflatoxin-associated mutational hotspot at codon 249 in the p53 tumor suppressor gene occurs in hepatocellular carcinomas from Mexico. Carcinogenesis 17, 1007-12 (1996).

5. Williams, J.H. et al. Human aflatoxicosis in developing countries: a review of toxicology, exposure, potential health consequences, and interventions. Am J Clin Nutr 80, 1106-22 (2004).

6. Gursoy-Yuzugullu, O., Yuzugullu, H., Yilmaz, M. \& Ozturk, M. Aflatoxin genotoxicity is associated with a defective DNA damage response bypassing p53 activation. Liver Int 31, 561-71 (2011).

7. Ribeiro, D.H., Ferreira, F.L., da Silva, V.N., Aquino, S. \& Correa, B. Effects of aflatoxin B(1) and fumonisin $\mathrm{B}(1)$ on the viability and induction of apoptosis in rat primary hepatocytes. Int J Mol Sci 11, 1944-55 (2010).

8. Wu, H.C. \& Santella, R. The Role of Aflatoxins in Hepatocellular Carcinoma. Hepat Mon 12, e7238 (2012).

9. Kew, M.C. Aflatoxins as a cause of hepatocellular carcinoma. J Gastrointestin Liver Dis 22, 305-10 (2013).

10. Zhang, Y.J. et al. Aflatoxin B1 and polycyclic aromatic hydrocarbon adducts, p53 mutations and p16 methylation in liver tissue and plasma of hepatocellular carcinoma patients. Int J Cancer 119, 985-91 (2006).

11. Zhang, Y.J. et al. Global hypomethylation in hepatocellular carcinoma and its relationship to aflatoxin $\mathrm{B}(1)$ exposure. World J Hepatol 4, 169-75 (2012).

12. Hsu, I.C. et al. Mutational hotspot in the p53 gene in human hepatocellular carcinomas. Nature 350, 427-8 (1991).

13. Hussain, S.P., Schwank, J., Staib, F., Wang, X.W. \& Harris, C.C. TP53 mutations and hepatocellular carcinoma: insights into the etiology and pathogenesis of liver cancer. Oncogene 26, 2166-76 (2007).

14. Zhang, Y.J. et al. Inactivation of the DNA repair gene O6-methylguanine-DNA methyltransferase by promoter hypermethylation and its relationship to aflatoxin B1-DNA adducts and p53 mutation in hepatocellular carcinoma. Int J Cancer 103, 440-4 (2003).

15. Valinluck, V. et al. Oxidative damage to methyl-CpG sequences inhibits the binding of the methyl-CpG binding domain (MBD) of methyl-CpG binding protein 2 (MeCP2). Nucleic Acids Res 32, 4100-8 (2004).

16. Siomi, H. \& Siomi, M.C. Posttranscriptional regulation of microRNA biogenesis in animals. Mol Cell 38, 323-32 (2010).

17. Fornari, F. et al. MiR-221 controls CDKN1C/p57 and CDKN1B/p27 expression in human hepatocellular carcinoma. Oncogene 27, 5651-61 (2008).

18. Valencia-Quintana, R. et al. The microRNAs as potential biomarkers for predicting the onset of aflatoxin exposure in human beings: a review. Front Microbiol 5, 102 (2014).

19. Takagi, S., Nakajima, M., Mohri, T. \& Yokoi, T. Post-transcriptional regulation of human pregnane X receptor by micro-RNA affects the expression of cytochrome P450 3A4. J Biol Chem 283, 9674-80 (2008).

20. Georges, S.A. et al. Coordinated regulation of cell cycle transcripts by p53-Inducible microRNAs, miR-192 and miR-215. Cancer Res 68, 10105-12 (2008).

21. Kamburov, A., Wierling, C., Lehrach, H. \& Herwig, R. ConsensusPathDB--a database for integrating human functional interaction networks. Nucleic Acids Res 37, D623-8 (2009).

22. Mosmann, T. Rapid colorimetric assay for cellular growth and survival: application to proliferation and cytotoxicity assays. J Immunol Methods 65, 55-63 (1983).

23. van Breda, S.G. et al. Epigenetic mechanisms underlying arsenic-associated lung carcinogenesis. Arch Toxicol (2014).

24. Young, M.D. et al. ChIP-seq analysis reveals distinct $\mathrm{H} 3 \mathrm{~K} 27 \mathrm{me} 3$ profiles that correlate with transcriptional activity. Nucleic Acids Res 39, 7415-27 (2011).

25. Edgar, R., Domrachev, M. \& Lash, A.E. Gene Expression Omnibus: NCBI gene expression and hybridization array data repository. Nucleic Acids Res 30, 207-10 (2002).

26. Eijssen, L.M. et al. User-friendly solutions for microarray quality control and pre-processing on ArrayAnalysis.org. Nucleic Acids Res 41, W71-6 (2013).

27. Gautier, L., Cope, L., Bolstad, B.M. \& Irizarry, R.A. affy--analysis of Affymetrix GeneChip data at the probe level. Bioinformatics 20, 307-15 (2004).

28. Gentleman, R.C. et al. Bioconductor: open software development for computational biology and bioinformatics. Genome Biol 5, R80 (2004).

29. Rieswijk, L., Lizarraga, D., Brauers, K.J., Kleinjans, J.C. \& van Delft, J.H. Characterisation of cisplatininduced transcriptomics responses in primary mouse hepatocytes, HepG2 cells and mouse embryonic stem cells shows conservation of regulating transcription factor networks. Mutagenesis 29, 17-26 (2014). 
30. Smyth, G.K. Limma: linear models for microarray data., 397-420 (Springer, New York, 2005).

31. Lopez-Romero, P. Pre-processing and differential expression analysis of Agilent microRNA arrays using the AgiMicroRna Bioconductor library. BMC Genomics 12, 64 (2011).

32. Hsu, S.-D. et al. miRTarBase: a database curates experimentally validated microRNA-target interactions. Nucleic Acids Research 39, D163-D169 (2011).

33. Lu, T.P. et al. miRSystem: an integrated system for characterizing enriched functions and pathways of microRNA targets. PLoS ONE 7, e42390 (2012).

34. Davis, A.P. et al. The Comparative Toxicogenomics Database: update 2013. Nucleic Acids Res 41, D1104-14 (2013).

35. Caiment, F., Tsamou, M., Jennen, D. \& Kleinjans, J. Assessing compound carcinogenicity in vitro using connectivity mapping. Carcinogenesis 35, 201-7 (2014).

36. Gao, J. et al. Integrating and annotating the interactome using the MiMI plugin for cytoscape. Bioinformatics 25, 137-8 (2009).

37. Kutmon, M., Kelder, T., Mandaviya, P., Evelo, C.T. \& Coort, S.L. CyTargetLinker: a cytoscape app to integrate regulatory interactions in network analysis. PLoS ONE 8, e82160 (2013).

38. Warsow, G. et al. ExprEssence--revealing the essence of differential experimental data in the context of an interaction/regulation net-work. BMC Syst Biol 4, 164 (2010).

39. Chang, T.C. et al. Transactivation of miR-34a by p53 broadly influences gene expression and promotes apoptosis. Mol Cell 26, 745-52 (2007).

40. Wang, Z. et al. Associations of miR-499 and miR-34b/c Polymorphisms with Susceptibility to Hepatocellular Carcinoma: An Evidence-Based Evaluation. Gastroenterol Res Pract 2013, 719202 (2013).

41. Xu, Y. et al. A potentially functional polymorphism in the promoter region of $\mathrm{miR}-34 \mathrm{~b} / \mathrm{c}$ is associated with an increased risk for primary hepatocellular carcinoma. Int J Cancer 128, 412-7 (2011).

42. Zhang, J. et al. MiRNA-99a directly regulates AGO2 through translational repression in hepatocellular carcinoma. Oncogenesis 3, e97 (2014).

43. Wang, B. et al. TGFbeta-mediated upregulation of hepatic miR-181b promotes hepatocarcinogenesis by targeting TIMP3. Oncogene 29, 1787-97 (2010).

44. Li, J., Wang, Y., Yu, W., Chen, J. \& Luo, J. Expression of serum miR-221 in human hepatocellular carcinoma and its prognostic significance. Biochem Biophys Res Commun 406, 70-3 (2011).

45. Karakatsanis, A. et al. Expression of microRNAs, miR-21, miR-31, miR-122, miR-145, miR-146a, miR200c, miR-221, miR-222, and miR-223 in patients with hepatocellular carcinoma or intrahepatic cholangiocarcinoma and its prognostic significance. Mol Carcinog 52, 297-303 (2013).

46. Rowinsky, E.K. Signal events: Cell signal transduction and its inhibition in cancer. Oncologist 8 Suppl 3, 5-17 (2003).

47. Hanahan, D. \& Weinberg, Robert A. Hallmarks of Cancer: The Next Generation. Cell 144, 646-674 (2011).

48. Hannigan, G.E., McDonald, P.C., Walsh, M.P. \& Dedhar, S. Integrin-linked kinase: not so 'pseudo' after all. Oncogene 30, 4375-85 (2011).

49. Lange, A. et al. Integrin-linked kinase is an adaptor with essential functions during mouse development. Nature 461, 1002-6 (2009).

50. Oneyama, C. et al. MicroRNA-mediated upregulation of integrin-linked kinase promotes Src-induced tumor progression. Oncogene 31, 1623-35 (2012). 
0

$\bigcirc$

O

0

00

()

0

\section{Chapter 6}

$\mathrm{O}$

Persistent microRNA-gene networks in () primary human hepatocytes upoß withdrawal of aflatoxin B1 exposuregre; related to hepatocellular carcinoma.
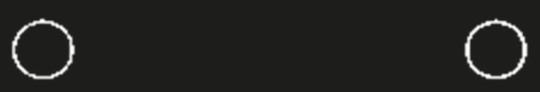

$\bigcirc$ Submitted
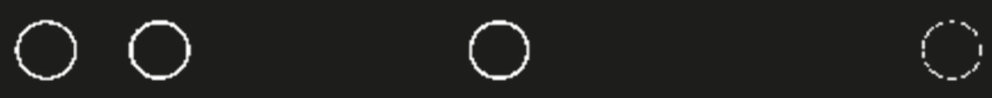

0

(C) Rieswijk $\mathbf{L}$

Claessen S.M.H.

Bekerso.

Therroussen D.H.J.

$\bigcirc \bigcirc$

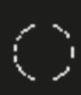

Jennen D.G.J.

de Kok M.C.M.

0


Kleinjans J.C.S. van Breda S.G.J.

$$
\text { i }
$$




\section{$\underline{\text { Abstract }}$}

Hepatocellular carcinoma (HCC), one of the most common cancer types, is a multi-factorial disease. One strongly associated factor is oral exposure to the food-contaminant aflatoxin B1 (AFB1). A clear link between AFB1-induced p53 mutations and HCC exists, however less is known about the association with microRNA expression changes. In particular, irreversible adverse effects that may occur at the transcriptomic level following repetitive exposure have not been investigated yet. Therefore, in this study, we aimed to dissect persistent changes in expression of microRNA-directed regulatory networks from transient transcriptome modifications contributing to AFB1-induced HCC. Primary human hepatocytes were exposed to $1 \mu \mathrm{M}$ of AFB1 for 5 days followed by a 3 day wash-out period. Whole genome transcriptomic analysis and microRNA expressions were analyzed applying microarray technologies. By evaluating genes which remain significantly expressed into the same direction after the 3-day wash-out period persistent AFB1-induced and HCC-related biological processes could be identified. Persistent genes could be linked with "Drug and energy metabolism" and "Pre-, post- and transcriptional regulation of gene expression" related processes. Especially, two HCC-related microRNAs, hsa-miR-34b-5p and hsa-miR-222-3p, which are persistently expressed, seem to regulate genes involved in these very characteristic AFB1-induced processes referring to cancer-associated metabolism changes and regulation of gene expression. Moreover, hsa-miR-34a-5p, hsa-miR-96-5p and hsa-miR-30a-3p which become differentially expressed only upon terminating AFB1 exposure, seem to regulate persistently expressed genes. The findings within this study therefore contribute to a better understanding of the AFB1-induced onset of HCC by causing persistent effects on microRNA and gene expression. 


\section{Introduction}

Hepatocellular carcinoma (HCC) is one of the most lethal and prevalent types of cancer worldwide. HCC is a very complex disease which affects all segments of the world population. However regional differences exist between ethnicities ${ }^{1}$. These differences are related with specific etiological factors such as chronic hepatitis $\mathrm{B}$ and $\mathrm{C}$ viral infection, chronic alcohol consumption, and intake of aflatoxin-B1-contaminated food.

Aflatoxin B1 (AFB1), a class I hepatocarcinogenic mycotoxin (International Agency for Research on Cancer (IARC) ${ }^{2}$ produced by a variety of Aspergillus fungi strains ${ }^{3}$, frequently contaminates corn, soybeans, and peanuts. It is therefore not surprising that in developing countries, where the intake of these food sources is high, the prevalence of HCC is also high ${ }^{1}$.

After ingestion of the fungal toxin, AFB1, hepatic bioactivation by phase I cytochrome P450 (CYP) enzymes CYP1A2 and CYP3A4 is required to convert the indirect carcinogen into toxic intermediates, such as exo-8,9-epoxide, the main and extremely active epoxide metabolite of $\mathrm{AFB} 1{ }^{4}$.

The irreversible DNA adducts (mainly guanine and FAPY adducts) caused by these AFB1 metabolites, are mutagenic and may therefore increase the risk of developing HCC. AFB1 is known to introduce a specific transversion mutation $(G \rightarrow T)$ in codon 249 of the p53 tumor suppressor gene ${ }^{5}$. A mutation in the p53 gene may lead to inactivation of the gene and therefore affect cell proliferation ${ }^{1}$. Many genetic and epigenetic alterations may additionally occur eventually leading to the deregulation of key oncogenes and tumour-suppressor and other important downstream genes such as beta-catenin, ErbB receptor family members, MET and its ligand hepatocyte growth factor (HGF), p16(INK4a), E-cadherin and cyclooxygenase 2 (COX2) ${ }^{1}$.

Besides genotoxic stress, AFB1 may also induce oxidative stress ${ }^{6}$. The detoxification of AFB1 is mediated by glutathione S-transferase. High levels of AFB1 may therefore lead to depletion of glutathione, thus indirectly resulting in high levels of reactive oxygen species. This increase in oxidative stress might affect also other processes such as glycolysis, electron transport chain activity involved in ATP production, protein synthesis and lipid peroxidation ${ }^{7}$. Subsequently, cytotoxicity ${ }^{8}$, cellular death by apoptosis (preferential binding for mitochondrial DNA), and necrosis ${ }^{9}$ have been reported to be induced by AFB1.

Whether AFB1 causes precarcinogenic effects on microRNA and gene expression is less clear. MicroRNAs are small non-coding RNA molecules ( 22 nucleotides long) which are known to regulate gene expression at the post-transcriptional level. It is well known that microRNA expression levels are altered in HCC ${ }^{10}$. It is of interest that several studies have demonstrated that changes on the microRNA level in an early phase (before the onset of cancer) already represent the microRNA expression signature characteristic for the disease itself ${ }^{11}$. Therefore chemically-induced early effects on the microRNA level may provide insight into the initiating mechanisms of HCC and consequently, may be predictive for the further development of HCC.

MicroRNAs expression is hypothesized to be relatively sensitive to environmental carcinogens 12,13. This is related to the fact that microRNA gene expression is activated as a consequence of DNA damage ${ }^{14}$. Whereas early microRNA alterations induced by low exposure levels of carcinogens can still be interpreted as adaptive mechanisms that activate defensive genes involved in metabolic detoxification, DNA and protein repair, and apoptosis activation, exceedingly higher doses after long-term exposure may induce persistent alterations which play 
a pathogenic role in HCC development ${ }^{15}$. Hypothetically, effects on the microRNA level would therefore persist upon withdrawal of the carcinogen exposure. For a relatively small set of environmental carcinogens, in relation to carcinogenesis, microRNA expression patterns appear to show a certain degree of overlap among compounds ${ }^{16}$. This indicates that microRNAs are involved in a common stress response to a certain group of compounds with potentially the same endpoints of toxicity.

Few studies have focused on the effect of AFB1 exposure on microRNA and gene expression in relation to HCC 17-19. Some microRNAs that were found in an in vivo study on AFB1-treated rats $(1.5 \mathrm{mg} / \mathrm{kg}$ for 3 days) suffering from liver injury, are members of the cancer-related rnomiR-17-92 cluster and rno-miR-34a ${ }^{19}$.Several other microRNAs have already been associated with HCC development (human tissues) in relation to AFB1 exposure ${ }^{17}$, for example microRNA-148 (gene targets: PXR and CYP3A4 ${ }^{20}$ ) and microRNA-192 (involved in NER repair pathway genes $\left.{ }^{21}\right)$.

However, till date, no study has focused on the possible persistence of AFB1-induced microRNA-gene interaction networks and their relevance for the development of HCC upon termination of the carcinogen exposure.

Therefore, in the present study, we hypothesize that persistent AFB1-induced microRNA/gene expression changes play a role in biological processes contributing to the development of HCC. To mimic the chronic exposure leading to HCC, repeated treatment is essential since the half-life of AFB1 by itself is 30 minutes and that of AFB1-DNA adducts in animals is less than $24 \mathrm{~h} \mathrm{22,23}$. Therefore, primary human hepatocytes (PHH) were exposed repetitively to $1 \mu \mathrm{M}$ of $\mathrm{AFB} 1$ for 5 days. To evaluate the persistence of induced transcriptomic changes, this carcinogenic challenge was followed by a 3 day wash-out period. Whole genome transcriptomic analysis was performed using Affymetrix whole genome gene expression microarrays in combination with microRNA analyses using Agilent microRNA microarrays. Biological interaction networks were created within Cytoscape ${ }^{24}$ using the biological process Gene Ontology categories (GO) within ConsensusPathDB 25. Differentially expressed transcripts, which represent known targets of differentially expressed microRNAs, were investigated in more detail.

\section{Materials and methods}

\section{Cell culture, dose determination and aflatoxin B1 treatment}

Cryopreserved primary human hepatocytes (PHH) were obtained from Life Technologies. Cells were cultured in pre-coated 24 -well plates $(700,000$ cells $/ \mathrm{ml})$ in a 2-layer collagen sandwich (A11428-02, Gibco), according to the supplier's protocol (Invitrogen, 2012). The following culture media were used: Hepatocyte Thawing Medium (HTM) for thawing (CM7500, Gibco), Williams' Medium E (1x, no phenol red) (A1217601, Gibco) + Cell Maintenance supplement B kit (CM4000, Gibco) for plating and incubation. After quick thawing in a water bath at $37^{\circ} \mathrm{C}$, viability of the cells was checked by a Trypan blue (CAS no. 72-57-1, Sigma-Aldrich) exclusion test as instructed in the supplier's protocol (Invitrogen, 2012). All viability scores after thawing were in agreement with those listed by the supplier. Before AFB1 treatment, cells were allowed to acclimatize for 48 hours. This is needed for the hepatocytes to restore an in vivo like cellular configuration and enzyme expression levels as optimally as possible. An AFB1 dose causing moderate (IC40) cytotoxicity after 5 days of repetitive daily exposure, was established by means of the MTT assay ${ }^{26}$. Furthermore, crucial liver function enzymes such as lactate dehydrogenase $(\mathrm{LDH})$ and alanine transaminase (ALT) were measured. LDH and ALT were spectrophotometrically determined on a Cobas 8000 
Modular Analyser (Roche Diagnostics, Basel, Switzerland). Based on this data, an incubation concentration of $1 \mu \mathrm{M}$ of AFB1 (CAS Number 1162-65-8, Sigma-Aldrich) was selected for the main experiment. Vehicle control cells were treated with $0.5 \%$ of dimethylsulfoxide (DMSO) (CAS number 67-68-5, Sigma-Aldrich).

To reduce the possible effect of inter-donor variability in AFB1 response, $\mathrm{PHH}$ from three donors of different sex, ethnicity, age, body mass index and behavioral status regarding smoking, alcohol and drug use (see Supplementary Table 6.1) were pooled. Cells were cultured in triplicate in 24 wells in a collagen sandwich layer system. One part of the cell lysates was harvested following 5 days of repetitive daily exposure to AFB1 or the vehicle control, and the other part of the cell lysates was analyzed following an additional 3 day wash-out period.

\section{Gene expression analyses}

\section{Total RNA isolation and quality control}

At the end of treatment, medium was removed and PHH were harvested in Qiazol (Qiagen). Total RNA was isolated using a miRNeasy Mini Kit (Qiagen) according to the manufacturer's protocol and followed by DNase I (Qiagen) treatment. Upon purification, RNA concentrations were measured by means of a NanoDrop ${ }^{\circledR}$ ND-1000 spectrophotometer (Thermo Scientific) at 260 and $280 \mathrm{~nm}$. RNA quality and integrity were assessed by using automated gel electrophoresis on an Agilent 2100 Bioanalyzer system (Agilent Technologies). Only RNA samples which showed clear $18 \mathrm{~S}$ and $28 \mathrm{~S}$ peaks and with an RNA integrity number (RIN) higher than 8 , were used. Samples were stored at $-80^{\circ} \mathrm{C}$ until RNA hybridization.

\section{Labelling, hybridization, re-annotation}

High-density oligonucleotide GeneChips from Affymetrix were used to measure gene expression levels (Human Genome U133 Plus 2.0 array (604,258 probes)). Targets for these arrays were prepared, hybridized and scanned according to the Affymetrix protocol (Affymetrix).Normalization quality controls appeared to be within acceptable limits for almost all chips.

The mRNA microarray data have been deposited in NCBI's Gene Expression Omnibus (GEO) ${ }^{27}$ and are accessible through accession number GSE67002 and GSE71542.

\section{Re-annotation, normalization and data filtering}

The Arrayanalysis.org web service was used for quality control ${ }^{28}$. All microarrays appeared of high quality, except one which was thereupon omitted from the analyses. CEL files were imported into R v2.15.3 (http://www.r-project.org) using the "affy" library 29 within BioConductor (v2.9) ${ }^{30}$. Probe re-annotation, normalization and data filtering was performed as previously described ${ }^{31}$.

\section{Selection of differentially expressed genes (DEGs)}

Differentially expressed genes (DEGs) were selected using the linear model for microarrays (LIMMA) approach ${ }^{32}$. The resulting p-values were FWER-corrected using the False Discovery Rate (FDR) method. The following criteria were applied: (1) average expression in at least one of the experimental groups $>6$ (log2-scale), (2) a FDR-corrected p-value $<0.05$ obtained through a moderated t-test, and (3) for the three replicates an average absolute fold change (FC) of 1.5 or higher (i.e., average $\log 2$ ratio of $<-0.58$ or $>0.58$ ). 


\section{Selecting AFB1-induced, HCC-related DEGs}

To evaluate the plausibility of transcriptome changes observed in vitro, with respect to human disease risks, results were benchmarked against gene expression data retrieved from samples from hepatocellular carcinoma patients. Therefore, a comparison was made with a HCC signature (based on 8934 genes which were different between HCC versus healthy human liver) created by Caiment et al. (2014) ${ }^{33}$ by using publically available gene expression data sets from ArrayExpress (HCC versus healthy human liver). The resulting list was complemented with 4987 genes that have previously been associated with effects of AFB1 exposure (obtained from curated data found in the Comparative Toxicogenomics Database (CTD) (http://ctdbase.org/) ${ }^{34}$. Two groups of AFB1-induced genes were created; one group representing a priori known AFB1-associated, HCC-related genes and a second group containing HCC-related genes which have not yet been associated with molecular mechanisms of AFB1-induced carcinogenesis, and thus, present novel findings.

Differentially expressed genes found after the 5-day AFB1 treatment period and after the 3-day wash-out period, were compared in order to identify DEGs remaining after the 3-day wash-out period, DEGs which were no longer present after the 5-day exposure period, and transcripts only emerging after the 3-day wash-out period. Respective DEGs were then imported into ConsensusPathDB for biological process (GO) analysis ${ }^{25}$.

\section{MicroRNA expression analyses}

Hybridization, normalization and selection of differentially expressed microRNAs (DE-miRs)

MicroRNA expression profiling was performed using Agilent Sureprint G3 Unrestricted Human miRNA V19 $8 \times 60 \mathrm{~K}$ microarrays. The hybridization was performed following standard protocols, after which the microarray slides were washed and scanned using a DNA microarray scanner (Agilent Technologies). The scanned images were converted into TXT files using the Feature Extraction Software v10.7.3.1 from Agilent Technologies, which were imported into R 2.15.3 (http://www.r-project.org) for quality control using an in-house developed pipeline. Filtering and normalization was performed using AgiMicroRna ${ }^{35,36}$. Total gene signals were $\log 2$-transformed and quantile-normalized. The selection criteria for determining differentially expressed microRNAs (DE-miRs) were identical to those for DEGs. The DE-miRs between the 5-day treatment period and the 3-day wash-out period were then compared in order to identify DE-miRs persistently expressed, reversibly expressed and only expressed after washout.

The microRNA microarray data have been deposited in NCBI's Gene Expression Omnibus (GEO) 27 and are accessible through accession number GSE67003 and GSE71540.

\section{Target information of DE-miRs}

To understand the biological function of DE-miRs, target information was retrieved using a database containing over 50,000 experimentally validated microRNA-mRNA interactions (miRTarBase - Homo sapiens, release 4.5, miRBase version 20).

\section{Biological interpretation of DEGs and DE-miRs by biological process (GO) analysis}

By using ConsensusPathDB 25 significant biological processes $(G O)$ ( $p$-value $<0.01$ ) were selected and compared in order to identify unique GO processes. In this context, unique GO processes were identified specific for a priori known or novel AFB1-responsive and HCCrelated genes which were persistently expressed, only expressed during the 5-day exposure 
period or only expressed during the 3-day wash-out period. All genes involved in these unique GO processes were extracted and summarized within one network list. Gene-gene interactions and microRNA-gene interactions were retrieved using the MiMI plugin (with the option for "Interactions among query genes (include at least 2 genes) ${ }^{37}$ ) in combination with the CyTargetLinker plugin (based on the regulatory interaction network (RIN) of miRTarBase v.4.5 ${ }^{38}$ ) within Cytoscape. General terms representing the affected GO processes were then assigned to the clusters of genes within the networks. MicroRNA-directed regulations were also added to the networks. The MultiColoredNodes plugin ${ }^{39}$ was used for visualization of the observed expression values following AFB1 exposure.

\section{Results}

\section{Gene expression changes}

Exposure of a pool of $\mathrm{PHH}$ to $1 \mu \mathrm{M}$ of AFB1 resulted in statistically significant changes in gene expressions. After 5 days of repetitive AFB1 exposure, 2555 genes (1399 up regulated and 1156 down regulated) were significantly expressed (see part A of Supplementary Table 6.2). 1965 genes (896 up regulated and 1069 down regulated) were found to be significantly expressed after day 3 of the wash-out period (see part B of Supplementary Table 6.2). 1388 out of the 2555 significantly changed genes remained significantly expressed into the same direction after the 3-day wash-out period (see Figure-6.1). 458 out of these 1388 transcripts increased in their magnitude of expression (i.e. fold change of up and down regulated genes become more positive resp. more negative). 930 out of these 1388 DEGs decreased in their magnitude of expression (i.e. fold change of up and down regulated genes becomes less positive resp. less negative) (see part $\mathrm{C}$ of Supplementary Table 6.2).

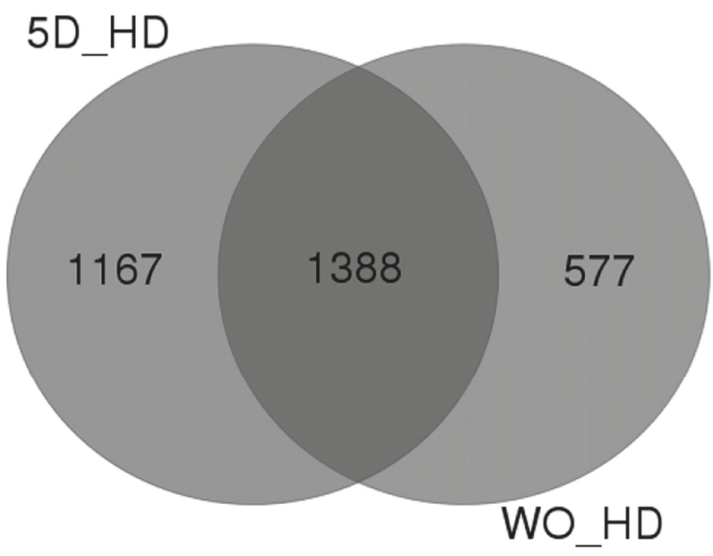

Figure 6.1: Venn diagram depicting that 1388 out of the 2555 significantly changed genes after 5 days of exposure (5D_HD) appeared to be persistently expressed after the 3 days of the wash-out period (WO_HD).

From the 2555 DEGs found after the 5-day treatment period, 377 appeared to be a priori known to be associated with AFB1-induced toxicity and related to HCC while 450 DEGs related to HCC, have not yet been reported in relation to AFB1 exposure and thus are considered novel findings (see part D and E of Supplementary Table 6.2 respectively and Table-6.1). Likewise, from the 1965 DEGs found after day 3 of the wash-out period, 279 were previously associated with AFB1 exposure and HCC while 246 have not been described in connection with AFB1 exposure before and therefore present newly associated genes (see part F and G of Supplementary Table 6.2 and Table-6.1). 234 DEGs of known AFB1-associated 
and HCC-related genes and 180 newly detected DEGs were observed to remain expressed after the 3-day wash-out period (see part H and I of Supplementary Table 6.2 and Table-6.1). 413 DEGs appeared to be transiently expressed (see part H and I of Supplementary Table 6.2 and Table-6.1). 45 a priori known AFB1-associated resp. 66 novel AFB1-induced HCC-related DEGs appeared to be affected only after the 3-day wash-out period (see part $\mathrm{H}$ and I of Supplementary Table 6.2 and Table-6.1).

Table 6.1: Table of differentially expressed genes (DEGs) which remained expressed after the 3-day wash-out period, disappeared after the 5-day exposure period and were only expressed after the 3-day wash- out of a priori known AFB1-induced, HCC-related and novel AFB1-induced, HCC-related DEGs.

DEGs remain after the 3-day wash-out period
DEGs disappear after the 5day exposure period
DEGs emerge only after 3-day wash-out period

\begin{tabular}{|c|c|c|c|c|c|c|}
\hline & $\begin{array}{l}\text { Previously related to } \\
\text { AFB1 }\end{array}$ & Novel findings & $\begin{array}{l}\text { Previously related to } \\
\text { to } A F B 1\end{array}$ & Novel findings & $\begin{array}{l}\text { Previously related } \\
\text { to } A F B 1\end{array}$ & Novel findings \\
\hline Total & 234 & 180 & 143 & 270 & 45 & 66 \\
\hline $\begin{array}{l}\text { Down- } \\
\text { regulated }\end{array}$ & 112 & 67 & 34 & 57 & 18 & 22 \\
\hline $\begin{array}{l}\mathrm{Up}- \\
\text { regulated }\end{array}$ & 122 & 113 & 109 & 213 & 27 & 44 \\
\hline
\end{tabular}

\section{MicroRNA expression changes}

Exposure of PHH to $1 \mu \mathrm{M}$ of AFB1 resulted in a set of 19 differentially expressed microRNAs (DE-miRs) after the 5 day treatment period while $17 \mathrm{DE}$-miRs were found after the 3 day wash-out period (see part J of Supplementary Table 6.2 for the expression values).

Four of the DE-miRs (up regulated: hsa-miR-222-3p, hsa-miR-34b-5p, and hsa-miR-5100, down regulated: hsa-miR-4286) remained expressed after the 3-day wash-out period (same direction of change). $15 \mathrm{DE}$-miRs appeared transiently expressed after the 5 day AFB1 treatment period while 13 appeared only expressed after the 3 day wash-out period (see part J of Supplementary Table 6.2 and Table-6.2).

Table 6.2: Table of differentially expressed microRNAs (DE-miRs) which remained expressed after the 3-day washout period, disappeared after the 5-day exposure period and were only expressed after the 3-day wash- out of a priori known AFB1-induced, HCC-related and novel AFB1-induced, HCC-related DEGs.

DE-miRs remain after the 3-day wash-out period
DE-miRs disappear after the 5-day exposure period
DE-miRs emerge only after 3-day wash-out period

\begin{tabular}{llll}
\hline Total & 4 & 15 & 13 \\
$\begin{array}{l}\text { Down- } \\
\text { regulated }\end{array}$ & 2 & 2 & 7 \\
$\begin{array}{l}\text { Up- } \\
\text { regulated }\end{array}$ & 2 & 13 & 6 \\
\hline
\end{tabular}

\section{Biological interpretation of DEGs and DE-miRs}

\section{Biological (GO) process analysis using ConsensusPathDB}

Persistent transcripts after the 3-day wash-out period are involved in 55 "Drug and energy metabolism" and 26 "Pre-, post- and transcriptional regulation of gene expression" related GO processes. DEGs which disappeared after the 5-day exposure period, appear involved in 41 "Stress response" and 45 "Cell cycle and DNA repair" related GO processes. DEGs activated 
only after 3 days of wash-out, were assigned to 2 "DNA damage response and replication" and 2 "Beta-oxidation" associated GO categories (see part K and L of Supplementary Table 6.2).

\section{Network visualization within Cytoscape}

Based on all 437 DEGs identified to be either persistently or transiently expressed, or to emerge during wash-out (see part $\mathrm{M}$ of Supplementary Table 6.2), a network was built using several plugins within Cytoscape.

Using the MiMI plugin, for 174 out of 437 DEGs, at least one interaction could be found with one or more of the other transcripts within the network. Also, 10 microRNA-gene interactions were successfully added to the network by using the Cy'TargetLinker plugin (see Figure 6.2).


Figure 6.2: Visualization of 174 out of 437 DEGs (represent all DEGs from the 98 and 73 significant Gene Ontology categories), with at least one interaction with one or more of the other genes within the network (using MiMI plugin). Ten microRNA-gene interactions were added to the network using the CyTargetLinker plugin. The genes within the network were then presented separating the a priori known from the novel AFB1-induced, HCC-related genes and the remaining transcripts after the 3-day wash-out period (depicted in yellow on left side) from the transcripts which are disappearing after 5 days of exposure (depicted in blue in middle part) and the ones only emerging after the wash-out (depicted in green on right side). The microRNAs were also separately clustered generating a more informative overview of the post-transcriptional regulation within this network (upper part of figure). Significant expression values of the respective transcripts were visualized using the MultiColordNodes plugin within Cytoscape (colors between -5 $($ downregulation=green) and 5 (upregulation $=$ red)). The white edges represent the gene-gene interactions while the red edges represent the microRNA-gene interactions. 


\section{Identification of regulatory microRNA-gene interactions within network}

The complexity of this network was reduced by highlighting the microRNA-gene interactions thus focusing on 31 DEGs (see Figure-6.3) (see part $\mathrm{N}$ of Supplementary Table 6.2). It is indicated that a relatively small proportion of all DEGs identified during and after AFB1 exposure, is regulated by microRNAs.



Figure 6.3: Visualization of 10 microRNAs and 31 differentially expressed target genes. MicroRNA-gene interactions were obtained using Cy TargetLinker. The genes within the subset network are presented in such a way that the $a$ prior known AFB1-induced, HCC-related genes are separated from the novel AFB1-induced, HCC-related genes and the remaining transcripts after the 3-day wash-out period (depicted in yellow on left side) from the transcripts which are disappearing after 5 days of exposure (depicted in blue in middle part) and the ones uniquely expressed after 3 days of wash-out (depicted in green on right side). The microRNAs were also separately presented in the upper part of the Figure Significant expression values of the respective transcripts were visualized using the MultiColordNodes plugin within Cytoscape (colors between -5 (downregulation=green) and 5 (upregulation=red)). The red edges represent the microRNA-gene interactions.

Apparently, the persistently expressed microRNA hsa-miR-222-3p regulates few persistently expressed target genes i.e. ACTB, PSMC (both important in "Drug and energy metabolism") and MACF1 (essential in "Pre-, post- and transcriptional regulation of gene expression"). Some DE-miRs activated after 3 days of wash-out, i.e. hsa-miR-30a-3p, hsa-miR-34a-5p and hsa-miR-96-5p, appear to target a relatively large number of persistent DEGs i.e. RPL27A, BIRC3, CHML, TXNRD1 and EVI5 (all substantial in "Drug and energy metabolism"), WDR77, KIF5B and NCBP2 (all important in "Pre-, post- and transcriptional regulation of gene expression"). DE-miR hsa-miR-1260b which was significantly down regulated after the 3 days wash-out period, seems to affect the persistent expression of its target gene SLC3A2 (essential in "Drug and energy metabolism"). The expression of this gene was the only one, 
within the microRNA-gene network, of which the level of expression was increased after the 3 days wash-out period.

Hsa-miR-34b-5p and hsa-miR-222-3p, both persistent DE-miRs, counter-regulate the expression of several transient DEGs i.e. HMGA1 (important in "Stress response"), RPL8, ILK, CDK4 and HIST1H4D (all essential in "Cell cycle and DNA repair"). Also the transiently changed i.e. ECT2, RRM2, S100A2, TPM1 (involved in "Stress response") and HIST1H4D, CFL1, CDK4, SNAP23 and AP2M1 (all significant in "Cell cycle and DNA repair") were regulated by DE-miRs hsa-miR-30a-3p, hsa-miR-34a-5p and hsa-miR-96-5p which appeared activated only after 3 days of wash-out.

\section{Discussion}

5 days of daily exposure to $1 \mu \mathrm{M}$ of AFB1 resulted in significant changes in gene as well as in microRNA expression patterns in PHH. Subsequently, 3 days of wash-out allowed the full clearance of AFB1 and AFB1-DNA adducts from the hepatocytes, in order to prevent effects occurring due to the presence of the carcinogen or its respective DNA adducts. After the wash-out period, more than $50 \%$ of genes were still significantly differentially expressed (Figure-6.1) and for one third the magnitude of expression increased. Based on existing gene expression associations or signatures, retrieved from CTD ${ }^{34}$ or composed by Caiment ${ }^{33}$, these transcripts could be linked with AFB1 exposure or HCC. As a result, several of these genes could be associated with the development of HCC and/or exposure to AFB1. More interesting also novel AFB1-responsive, HCC-related genes were identified (Table-6.1).

Hsa-miR-222-3p, hsa-miR-34b-5p, hsa-miR-5100 and hsa-miR-4286, appeared still expressed after the 3-day wash-out period (Figure-6.3 and Table-6.2). Two of these microRNAs (i.e. hsamiR-222-3p, hsa-miR-34b-5p) could be assigned to the regulatory network created within Cytoscape (Figure-6.3).

First, the function of these persistently expressed microRNAs and their relation with HCC is discussed. Then, the specific microRNA/gene interactions are discussed in more detail for the persistently expressed microRNAs and genes. Secondly the function of the microRNAs only activated during washout, their relation with HCC and interactions with persistently expressed are discussed.

Increased expression of hsa-miR-34b-5p has been shown to be directly induced by p53 in response to genotoxic stress (e.g. cyclo-phosphamide ${ }^{40}$ and benzo(a)pyrene ${ }^{41}$ ), acting upon downstream targets to promote cell cycle arrest or apoptosis ${ }^{42}$. However recent studies have also shown that expression of hsa-miR-34b-5p is largely independent of p53 status 43,44. Upregulated expression of hsa-miR-34b-5p has been observed with cancer progression in colon cancer, gastric cancer, renal cell carcinoma, lung squamous cell carcinoma, pediatric leukemia and rectal cancer ${ }^{44}$. Upregulated hsa-miR-34b-5p expression has not yet been described in HCC but it seems very likely that this microRNA also has a role in AFB1-induced development of HCC.

Upregulation of the second persistently expressed microRNA, hsa-miR-222-3p, has been frequently observed following exposure to genotoxic stress (e.g. arsenic ${ }^{45}$, TPA (12-Otetradecanoylphorbol-13-acetate) ${ }^{46}$, particulate matter (PM10) ${ }^{47,48}$, bisphenol A ${ }^{49}$, cyclophosphamide ${ }^{50}$ ) in in vitro ${ }^{45-47,49}$ as well as in vivo ${ }^{48,50}$ models. Deregulation of this oncogenic microRNA by AFB1 in HCC may result in promoted growth, clonogenic survival, migration and invasion of HCC cells ${ }^{51}$. We therefore conclude that the persistent expression pattern of this particular microRNA may very well be indicative for the initial phase of AFB1-induced HCC. 
Persistent AFB1-induced microRNA/gene interactions (Figure-6.3 and Table-6.1) were studied in more detail.

First the transcripts, which remained expressed after the 3-day wash-out period and which are involved in "Drug and energy metabolism", are discussed.

The beta actin (ACTB) gene, regulated by hsa-miR-222-3p (remained expressed after the 3-day wash-out period) and hsa-miR-221-3p (only expressed after the 5-day exposure period), codes for an abundant and highly conserved cytoskeleton structural protein that is widely distributed in all eukaryotic cells and plays critical roles in cell migration, cell division, embryonic development, wound healing, immune response and gene expression ${ }^{52}$. Possibly, abnormal overexpression and polymerization of ACTB and the resulting changes to the cytoskeleton are associated with the invasiveness and metastasis of cancers ${ }^{53-55}$. ACTB is up-regulated two- to three-fold in highly advanced stages of HCC. In this study, the expression of ACTB was dramatically increased ( $\sim$ five-fold) equaling the expression values as measured within HCC tissue. The microRNA-controlled persistent expression of ACTB in this study is a novel finding and is potentially related to the development of AFB1-associated HCC.

Proteasome (prosome, macropain) 26S subunit, ATPase, 4 (PSMC), a 26S proteasome coding gene regulated by hsa-miR-222-3p (remained expressed after the 3-day wash-out period), is a multicatalytic proteinase complex responsible for the cleavage of peptides in an ATP/ubiquitin-dependent and non-lysosomal process ${ }^{56}$. In HCC, an enhanced activation of protein degradation mechanisms has been observed. Selective degradation of critical proteins, including cell cycle inhibitors, may lead to increased cell proliferation important in HCC ${ }^{56}$. Persistent microRNA-directed upregulation of PSMC might therefore be relevant for the AFB1-induced onset of HCC.

Secondly the genes, which remained expressed after the 3-day wash-out period and which are involved in "Pre, post- and transcriptional regulation of gene expression", are discussed.

Microtubule-actin crosslinking factor 1 (MACF1), a large protein containing numerous spectrin and leucine-rich repeat (LRR) domains regulated by persistently expressed hsa-miR-222-3p, is a member of a family of proteins that form bridges between different cytoskeletal elements e.g. actin-microtubule interactions thereby acting in cell migration. Furthermore, MACF1 is involved in Wnt-induced TCF/ $\beta$-catenin-dependent transcriptional activation leading to increased expression of Wnt-responsive genes contributing in tumorigenesis. In HCC, this frequently occurs through mutations in the $\mathrm{N}$-terminal region of beta-catenin that stabilize the protein and permit an elevated level of constitutive transcriptional activation by betacatenin/TCF complexes. In this study, AFB1-induced persistently expressed MACF1, directed by hsa-miR-222-3p, might be crucial for the onset of HCC considering its role in the cancerrelated Wnt signaling pathway.

Remarkably, we also found microRNAs activated only during washout but capable of regulating persistently expressed genes. These were downregulated microRNAs hsa-miR-1260b and hsa-miR-505-3p and upregulated microRNAs hsa-miR-30a-3p, hsa-miR-34a-5p and hsamiR-96-5p (Figure-6.3 and Table-6.2). This effect on the microRNA level after withdrawal of the carcinogenic challenge, which seems to affect the persistently expressed genes in a late stage, may be of significance for AFB1-related hepatocarcinogenesis.

Upregulation of oncogenic hsa-miR-30a-3p is described for various cancer types 57 . Upregulation of hsa-miR-96-5p has been associated with cell proliferation and inhibition of apoptosis within HCC ${ }^{58}$. Upregulation of p53-regulated hsa-miR-34a-5p has been observed in a number of in vitro and in vivo studies following exposure to mainly genotoxic agents (e.g. 
cyclo-phosphamide ${ }^{40}$, benzo(a)pyrene 41 and N-ethyl-N-nitrosourea (ENU) ${ }^{59}$ ). More importantly, upregulation of rno-miR-34a-5p has been found in an in vivo study by Yang et al., 2014 in which rats were exposed for 3 days to $1.5 \mathrm{mg} / \mathrm{kg}$ of AFB1 until they suffered from liver injury.

Several genes persistently expressed after termination of AFB1 exposure and involved in "Drug and energy metabolism" appeared regulated by microRNAs only activated during washout:

The ribosomal protein L27a (RPL27A) gene, regulated by hsa-miR-30a-3p, codes for a protein which is a constituent of the $60 \mathrm{~S}$ subunit of a ribosomal protein. Overexpression of the ribosomal proteins, in colorectal cancer as well as HCC, is associated with enhanced colony formation and cell proliferation, resulting from rapid cell cycling ${ }^{60-62}$. The microRNAcontrolled persistent upregulation of RPL27A may therefore be vital for the onset of HCC induced by AFB1.

Another persistently expressed gene, baculoviral IAP repeat containing 3 (BIRC3), directed by hsa-miR-34a-5p, codes for a multi-functional protein involved the inhibition of apoptosis, inflammatory signaling and immunity, mitogenic kinase signaling and cell proliferation, as well as cell invasion and metastasis in HCC 63,64 . Persistent upregulation of microRNA-directed BIRC3 may therefore be very informative for the primary phase of AFB1-induced HCC.

Choroideremia-like (Rab escort protein 2) (CHML), a substrate-binding subunit (component A) of the Rab geranylgeranyltransferase (GGTase) complex coding gene targeted by hsa-miR96-5p, supports geranylgeranylation of most Rab proteins. Protein geranylgeranylation (GGylation) is a vital biochemical process for cellular localization of many signaling molecules e.g. Rho and Rab small GTPases and heterotrimeric GTPase gamma subunits. Previous studies have shown that GGylation is essential for cancer cell survival, growth, proliferation, migration and metastasis ${ }^{65,66}$. A clear role for the persistently expressed microRNA-targeted CHML in cancer has been described and therefore it might be very interesting in the preliminary occurrence of AFB1-directed HCC.

Thioredoxin reductase 1 (TXNRD1), a hsa-miR-34a-5p targeted member of the family of pyridine nucleotide oxidoreductases, is involved in the reduction of thioredoxins as well as other substrates, and plays a role in selenium metabolism and protection against oxidative stress ${ }^{67}$. Several studies have implicated that overexpression of thioredoxin is one of the enhancers of cancer cell growth, either through the direct stimulation of cancer cell growth or through the inhibition of cancer cell apoptosis ${ }^{67}$.

Another gene, which remained expressed after the 3-day wash-out period, ecotropic viral integration site 5 (EVI5), regulated by hsa-miR-96-5p, functions as a regulator of cell cycle progression by stabilizing the FBXO5 protein and promoting cyclin-A accumulation during interphase ${ }^{68}$. Furthermore, it may play a role in cytokinesis. Downregulation of EVI5, like in this study, has been associated with increased cell motility and invasiveness associated with HCC highlighting its significance in AFB1-induced onset of this disease ${ }^{68}$.

Also for "Pre, post- and transcriptional regulation of gene expression", persistently expressed genes were found which appeared regulated by microRNAs only activated during wash-out:

The protein coding gene WD repeat domain 77 (WDR77), which remained expressed after the 3 -day wash-out period and is regulated by hsa-miR-34a-5p, constitutes a non-catalytic component of the 20S PRMT5-containing methyltransferase complex, which modifies specific arginines to dimethylarginines in several spliceosomal Sm proteins and histones. In addition, it 
seems to regulate cell proliferation in prostate cancer ${ }^{69}$. Considering its role in other types of cancer, it seems likely that the persistently expressed WDR77 gene directed by hsa-miR-34a$5 \mathrm{p}$, plays a significant role in the initiation of AFB1-induced HCC ${ }^{69}$.

Persistently expressed kinesin family member 5B (KIF5B), targeted by hsa-miR-34a-5p, is involved in the microtubule-dependent motor activity which is required for normal distribution of mitochondria and lysosomes. In cancer cells, KIF5B is highly expressed and plays a significant role in growth and survival ${ }^{70}$. Since KIF5B has already been proven to be involved in crucial processes for the onset of HCC, it seems likely that persistent expression of this microRNA-targeted gene is important for the initiation of the disease.

Lastly, nuclear cap binding protein subunit 2, 20kDa (NCBP2), regulated by hsa-miR-34a-5p, codes for a component of the cap-binding complex $(\mathrm{CBC})$, which binds co-transcriptionally to the $5^{\prime}$ cap of pre-mRNAs and is involved in various processes such as pre-mRNA splicing, translation regulation, nonsense-mediated mRNA decay, RNA-mediated gene silencing (RNAi) by microRNAs (miRNAs) and mRNA export. Another member of the $\mathrm{CBC}$, eukaryotic initiation factor 4F (eIF4F), has already been associated with translational efficiency in tumorigenesis ${ }^{71}$. It seems therefore probably that microRNA-regulated NCBP2, which is persistently expressed, also plays a role in tumorigenesis and thereby in the early phase of AFB1-induced HCC.

Finally, previously known AFB1-associated transcripts which disappeared after the 5-day exposure period were observed to be involved in "Stress response", while newly found AFB1induced genes were observed to play a role in "Cell cycle and DNA repair". Both biological processes are known to be affected by AFB1 treatment ${ }^{4}$.

\section{Conclusion}

Our results indicate that persistent effects on the expression of microRNAs and genes involved in important signaling pathways for hepatocarcinogenesis and induced by the welldescribed human hepatocarcinogen AFB1, may be indicative for the early onset of HCC. These microRNA-targeted persistently expressed genes play a role in very characteristic AFB1induced processes related with cancer-associated metabolism changes and regulation of gene expression. Two previously HCC-associated microRNAs hsa-miR-34b-5p and hsa-miR-222-3p mainly seem to regulate these differentially expressed genes. Furthermore, hsa-miR-34a-5p, hsa-miR-96-5p and hsa-miR-30a-3p which become differentially expressed only upon terminating AFB1 exposure, can be associated with HCC. It is suggested that these rather late modifications of microRNA expression play an important role in the persistence of gene expression changes induced by AFB1. This concept needs to be further explored in future research in well-designed time-series experiments involving transcriptome and microRNAome analysis. By applying an integrative network approach, the current study was the first to identify carcinogen-induced persistently changed microRNA/gene networks which are indicative for the AFB1-induced onset of HCC.

\section{Supplementary data}

Supplementary Tables 6.1-6.2 are available upon request. 


\section{References}

1. Farazi, P.A. \& DePinho, R.A. Hepatocellular carcinoma pathogenesis: from genes to environment. Nat Rev Cancer 6, 674-87 (2006).

2 .

3.

4.

5.

6.

7.

8

9.

10.

11.

12.

13.

14.

15.

16.

17.

18.

19.

20.

21.

22.

23.

24.

25.

26.

27.

28.

Vainio, H., Coleman, M. \& Wilbourn, J. Carcinogenicity evaluations and ongoing studies: the IARC databases. Environ Health Perspect 96, 5-9 (1991).

Cary, J.W., Klich, M.A. \& Beltz, S.B. Characterization of aflatoxin-producing fungi outside of Aspergillus section Flavi. Mycologia 97, 425-32 (2005).

Bedard, L.L. \& Massey, T.E. Aflatoxin B1-induced DNA damage and its repair. Cancer Lett 241, 174-83 (2006).

Soini, Y. et al. An aflatoxin-associated mutational hotspot at codon 249 in the p53 tumor suppressor gene occurs in hepatocellular carcinomas from Mexico. Carcinogenesis 17, 1007-12 (1996).

Marra, M. et al. Molecular targets and oxidative stress biomarkers in hepatocellular carcinoma: an overview. J Transl Med 9, 171 (2011).

Williams, J.H. et al. Human aflatoxicosis in developing countries: a review of toxicology, exposure, potential health consequences, and interventions. Am J Clin Nutr 80, 1106-22 (2004).

Gursoy-Yuzugullu, O., Yuzugullu, H., Yilmaz, M. \& Ozturk, M. Aflatoxin genotoxicity is associated with a defective DNA damage response bypassing p53 activation. Liver Int 31, 561-71 (2011).

Ribeiro, D.H., Ferreira, F.L., da Silva, V.N., Aquino, S. \& Correa, B. Effects of aflatoxin B(1) and fumonisin $\mathrm{B}(1)$ on the viability and induction of apoptosis in rat primary hepatocytes. Int J Mol Sci 11, 1944-55 (2010).

Sun, J., Lu, H., Wang, X. \& Jin, H. MicroRNAs in hepatocellular carcinoma: regulation, function, and clinical implications. ScientificW orldJournal 2013, 924206 (2013).

Giordano, S. \& Columbano, A. MicroRNAs: new tools for diagnosis, prognosis, and therapy in hepatocellular carcinoma? Hepatology 57, 840-7 (2013).

Elamin, B.K., Callegari, E., Gramantieri, L., Sabbioni, S. \& Negrini, M. MicroRNA response to environmental mutagens in liver. Mutat Res 717, 67-76 (2011).

Izzotti, A. \& Pulliero, A. The effects of environmental chemical carcinogens on the microRNA machinery. International Journal of Hygiene and Environmental Health 217, 601-627 (2014).

Suzuki, H.I. \& Miyazono, K. Dynamics of microRNA biogenesis: crosstalk between p53 network and microRNA processing pathway. J Mol Med (Berl) 88, 1085-94 (2010).

Izzotti, A. et al. Dose-responsiveness and persistence of microRNA expression alterations induced by cigarette smoke in mouse lung. Mutat Res 717, 9-16 (2011).

Koufaris, C., Wright, J., Currie, R.A. \& Gooderham, N.J. Hepatic microRNA profiles offer predictive and mechanistic insights after exposure to genotoxic and epigenetic hepatocarcinogens. Toxicol Sci 128, 532-43 (2012).

Valencia-Quintana, R. et al. The microRNAs as potential biomarkers for predicting the onset of aflatoxin exposure in human beings: a review. Front Microbiol 5, 102 (2014).

Liu, Y.X. et al. MicroRNA-24 modulates aflatoxin B1-related hepatocellular carcinoma prognosis and tumorigenesis. Biomed Res Int 2014, 482926 (2014).

Yang, W. et al. Genome-wide miRNA-profiling of aflatoxin B1-induced hepatic injury using deep sequencing. Toxicol Lett 226, 140-9 (2014).

Takagi, S., Nakajima, M., Mohri, T. \& Yokoi, T. Post-transcriptional regulation of human pregnane X receptor by micro-RNA affects the expression of cytochrome P450 3A4. J Biol Chem 283, 9674-80 (2008).

Georges, S.A. et al. Coordinated regulation of cell cycle transcripts by p53-Inducible microRNAs, miR-192 and miR-215. Cancer Res 68, 10105-12 (2008).

Qian, G. et al. Physiologically based toxicokinetics of serum aflatoxin B1-lysine adduct in F344 rats. Toxicology 303, 147-51 (2013).

Lai, H. et al. Association between aflatoxin B1 occupational airway exposure and risk of hepatocellular carcinoma: a case-control study. Tumour Biol 35, 9577-84 (2014).

Cline, M.S. et al. Integration of biological networks and gene expression data using Cytoscape. Nat. Protocols 2, 2366-2382 (2007).

Kamburov, A., Wierling, C., Lehrach, H. \& Herwig, R. ConsensusPathDB--a database for integrating human functional interaction networks. Nucleic Acids Res 37, D623-8 (2009).

Mosmann, T. Rapid colorimetric assay for cellular growth and survival: application to proliferation and cytotoxicity assays. J Immunol Methods 65, 55-63 (1983).

Edgar, R., Domrachev, M. \& Lash, A.E. Gene Expression Omnibus: NCBI gene expression and hybridization array data repository. Nucleic Acids Res 30, 207-10 (2002).

Eijssen, L.M. et al. User-friendly solutions for microarray quality control and pre-processing on ArrayAnalysis.org. Nucleic Acids Res 41, W71-6 (2013). 
29. Gautier, L., Cope, L., Bolstad, B.M. \& Irizarry, R.A. affy--analysis of Affymetrix GeneChip data at the probe level. Bioinformatics 20, 307-15 (2004).

30. Gentleman, R.C. et al. Bioconductor: open software development for computational biology and bioinformatics. Genome Biol 5, R80 (2004).

31. Rieswijk, L., Lizarraga, D., Brauers, K.J., Kleinjans, J.C. \& van Delft, J.H. Characterisation of cisplatininduced transcriptomics responses in primary mouse hepatocytes, HepG2 cells and mouse embryonic stem cells shows conservation of regulating transcription factor networks. Mutagenesis 29, 17-26 (2014).

32. Smyth, G.K. Limma: linear models for microarray data. , 397-420 (Springer, New York, 2005).

33. Caiment, F., Tsamou, M., Jennen, D. \& Kleinjans, J. Assessing compound carcinogenicity in vitro using connectivity mapping. Carcinogenesis 35, 201-7 (2014).

34. Davis, A.P. et al. The Comparative Toxicogenomics Database: update 2013. Nucleic Acids Res 41, D1104-14 (2013).

35. Lopez-Romero, P. Pre-processing and differential expression analysis of Agilent microRNA arrays using the AgiMicroRna Bioconductor library. BMC Genomics 12, 64 (2011).

36. Coonen, M., Theunissen, D.H., Kleinjans, J.C. \& Jennen, D.G. MagiCMicroRna: a web implementation of AgiMicroRna using shiny. Source Code Biol Med 10, 4 (2015).

37. Gao, J. et al. Integrating and annotating the interactome using the MiMI plugin for cytoscape. Bioinformatics 25, 137-8 (2009).

38. Kutmon, M., Kelder, T., Mandaviya, P., Evelo, C.T. \& Coort, S.L. CyTargetLinker: a cytoscape app to integrate regulatory interactions in network analysis. PLoS ONE 8, e82160 (2013).

39. Warsow, G. et al. ExprEssence--revealing the essence of differential experimental data in the context of an interaction/regulation net-work. BMC Syst Biol 4, 164 (2010).

40. Gueta, K. et al. Teratogen-induced alterations in microRNA-34, microRNA-125b and microRNA-155 expression: correlation with embryonic p53 genotype and limb phenotype. BMC Dev Biol 10, 20 (2010).

41. Malik, A.I., Williams, A., Lemieux, C.L., White, P.A. \& Yauk, C.L. Hepatic mRNA, microRNA, and miR34a-target responses in mice after 28 days exposure to doses of benzo(a)pyrene that elicit DNA damage and mutation. Environ Mol Mutagen 53, 10-21 (2012).

42. Chang, T.C. et al. Transactivation of miR-34a by p53 broadly influences gene expression and promotes apoptosis. Mol Cell 26, 745-52 (2007).

43. Concepcion, C.P. et al. Intact p53-dependent responses in miR-34-deficient mice. PLoS Genet 8, e1002797 (2012).

44. Hiyoshi, Y. et al. Increased microRNA-34b and $-34 \mathrm{c}$ predominantly expressed in stromal tissues is associated with poor prognosis in human colon cancer. PLoS One 10, e0124899 (2015).

45. Marsit, C.J., Eddy, K. \& Kelsey, K.T. MicroRNA Responses to Cellular Stress. Cancer Research 66, 1084310848 (2006).

46. Chen, A. et al. Complementary analysis of microRNA and mRNA expression during phorbol 12-myristate 13-acetate (TPA)-induced differentiation of HL-60 cells. Biotechnol Lett 30, 2045-52 (2008).

47. Suarez, Y., Fernandez-Hernando, C., Pober, J.S. \& Sessa, W.C. Dicer dependent microRNAs regulate gene expression and functions in human endothelial cells. Circ Res 100, 1164-73 (2007).

48. Bollati, V. et al. Exposure to metal-rich particulate matter modifies the expression of candidate microRNAs in peripheral blood leukocytes. Environ Health Perspect 118, 763-8 (2010).

49. Tilghman, S.L. et al. Endocrine Disruptor Regulation of MicroRNA Expression in Breast Carcinoma Cells. PLOS ONE 7, e32754 (2012).

50. Alencar, A.J. et al. MicroRNAs are independent predictors of outcome in diffuse large B-cell lymphoma patients treated with R-CHOP. Clin Cancer Res 17, 4125-35 (2011).

51. Karakatsanis, A. et al. Expression of microRNAs, miR-21, miR-31, miR-122, miR-145, miR-146a, miR200c, miR-221, miR-222, and miR-223 in patients with hepatocellular carcinoma or intrahepatic cholangiocarcinoma and its prognostic significance. Mol Carcinog 52, 297-303 (2013).

52. Waxman, S. \& Wurmbach, E. De-regulation of common housekeeping genes in hepatocellular carcinoma. BMC Genomics 8, 243 (2007).

53. Guo, C., Liu, S., Wang, J., Sun, M.Z. \& Greenaway, F.T. ACTB in cancer. Clin Chim Acta 417, 39-44 (2013).

54. Fu, L.Y. et al. Suitable reference genes for real-time PCR in human HBV-related hepatocellular carcinoma with different clinical prognoses. BMC Cancer 9, 49 (2009).

55. Liu, S. et al. Selection of reference genes for RT-qPCR analysis in tumor tissues from male hepatocellular carcinoma patients with hepatitis B infection and cirrhosis. Cancer Biomark 13, 345-9 (2013).

56. Lee, J.S. et al. Classification and prediction of survival in hepatocellular carcinoma by gene expression profiling. Hepatology 40, 667-76 (2004).

57. Wang, T., Li, F. \& Tang, S. MiR-30a upregulates BCL2A1, IER3 and cyclin D2 expression by targeting FOXL2. Oncol Lett 9, 967-971 (2015).

58. Chen, R.X., Xia, Y.H., Xue, T.C. \& Ye, S.L. Suppression of microRNA-96 expression inhibits the invasion of hepatocellular carcinoma cells. Mol Med Rep 5, 800-4 (2012). 
Davidson, L.A. et al. n-3 Polyunsaturated fatty acids modulate carcinogen-directed non-coding microRNA signatures in rat colon. Carcinogenesis 30, 2077-84 (2009).

60. Kondoh, N. et al. Enhanced expression of S8, L12, L23a, L27 and L30 ribosomal protein mRNAs in human hepatocellular carcinoma. Anticancer Res 21, 2429-33 (2001).

61. Kim, J.H. et al. Over-expression of the ribosomal protein L36a gene is associated with cellular proliferation in hepatocellular carcinoma. Hepatology 39, 129-38 (2004).

62. Lai, M.-D. \& Xu, J. Ribosomal Proteins and Colorectal Cancer. Current Genomics 8, $43-49$ (2007).

63. $\mathrm{Hu}, \mathrm{Y}$. et al. Transcriptome profiling and genome-wide DNA binding define the differential role of fenretinide and all-trans RA in regulating the death and survival of human hepatocellular carcinoma Huh7 cells. Biochem Pharmacol 85, 1007-17 (2013).

64. Li, G., Chang, H., Zhai, Y.P. \& Xu, W. Targeted silencing of inhibitors of apoptosis proteins with siRNAs: a potential anti-cancer strategy for hepatocellular carcinoma. Asian Pac J Cancer Prev 14, 4943-52 (2013).

65. Mi, W. et al. Geranylgeranylation signals to the Hippo pathway for breast cancer cell proliferation and migration. Oncogene (2014)

66. Berndt, N., Hamilton, A.D. \& Sebti, S.M. Targeting protein prenylation for cancer therapy. Nat Rev Cancer 11, 775-91 (2011).

67. Karlenius, T.C. \& Tonissen, K.F. Thioredoxin and Cancer: A Role for Thioredoxin in all States of Tumor Oxygenation. Cancers (Basel) 2, 209-32 (2010).

68. Yasui, K. et al. EVI1, a target gene for amplification at 3q26, antagonizes transforming growth factor-betamediated growth inhibition in hepatocellular carcinoma. Cancer Sci (2015).

69. Peng, Y. et al. Distinct nuclear and cytoplasmic functions of androgen receptor cofactor p44 and association with androgen-independent prostate cancer. Proc Natl Acad Sci U S A 105, 5236-41 (2008).

70. Cardoso, C.M. et al. Depletion of kinesin 5B affects lysosomal distribution and stability and induces perinuclear accumulation of autophagosomes in cancer cells. PLoS One 4, e4424 (2009).

71. Pelletier, J., Graff, J., Ruggero, D. \& Sonenberg, N. Targeting the eIF4F translation initiation complex: a critical nexus for cancer development. Cancer Res 75, 250-63 (2015). 


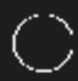

0

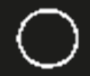

O

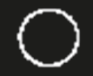

Chapter 7

00

)

$\bigcirc$

0

Aflatoxin B1 induces persistent epigenomic C) effects in primary human hepatocytes associated with hepatocellular carcinoma.

()

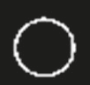

O
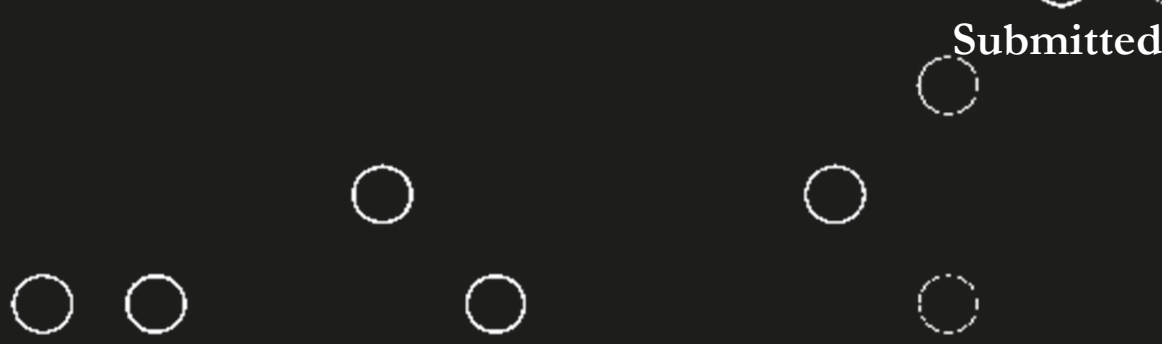

0

Rieswijk L..

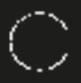

Clae@h S.M.H.

Bekers O.

(V)n (1e)wijnen M.

Theunissen D.H.J.

00

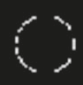

Jennen D.G.J.

de Kok M.C.M.

Kleinjans J.C.S.

van Breda S.G.J.

i.

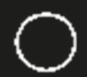




\section{Abstract}

Chronic exposure to aflatoxin B1 (AFB1) has, in certain regions in the world, been strongly associated with the development of hepatocellular carcinoma (HCC). AFB1 is a very potent hepatotoxic and carcinogenic mycotoxin which is frequently reported as a food contaminant. Epigenetic modifications provoked by environmental exposures, such as AFB1, may create a so called persistent "epigenetic memory" or "footprint". Deregulation of epigenetic mechanisms has actually been reported in HCC patients following AFB1 exposure; however, no attempts have yet been made to investigate early effects on the epigenome level which may be persistent on longer term, thereby possibly initiating carcinogenic events. In this study, we aim to identify methyl DNA-mRNA-interactions representative for a persistent epigenetic "footprint" associated with the early onset of AFB1-induced HCC. For this, primary human hepatocytes were exposed to $0.3 \mu \mathrm{M}$ of AFB1 for 5 days. Persistent epigenetic effects were measured 3 days after terminating the carcinogenic treatment. Whole genome DNA methylation changes and whole genome transcriptomic analysis were analyzed applying microarray technologies, and cross-omics interactions were evaluated. Upon combining transcriptomics data with results on DNA methylation, a range of persistent hyper- and hypomethylated genes was identified which also appeared affected on the transcriptome level. For six of the hypo-methylated and up-regulated genes, namely TXNRD1, PCNA, CCNK, DIAPH3, RAB27A and HIST1H2BF, a clear role in carcinogenic events could be identified. This study is the first to report on a carcinogen-induced persistent impact on the "epigenetic footprint" in relation with the transcriptome which could be indicative for the early onset of AFB1-related development of HCC. 


\section{Introduction}

Exposure to the highly potent carcinogenic and hepatotoxic mycotoxin aflatoxin B1 (AFB1) through the food chain presents a significant risk factor for the development of hepatocellular carcinoma (HCC) worldwide ${ }^{1,2}$. Especially populations in regions such as sub-Saharan Africa, Southeast Asia, and China are at risk of developing HCC due to the combination of high hepatitis B virus prevalence and largely uncontrolled dietary AFB1 exposure ${ }^{1}$. Of the 550,000 600,000 new HCC cases worldwide each year, about 25,000-155,000 may be attributable to AFB1 exposure ${ }^{1}$. Therefore, AFB1 exposure may have a causative role in $4.6-28.2 \%$ of all global HCC cases ${ }^{1}$. Against this background, a better understanding of the AFB1 mechanismof-action may be of value for underlining policy measures aiming at reducing exposure to this foodborne carcinogen.

Specific cytochrome P450 enzymes in the liver metabolize AFB1 into the highly reactive AFB1-8,9-epoxide, which may then bind to proteins and cause acute toxicity (aflatoxicosis) or to DNA to initiate mutations that over time increase the risk of HCC ${ }^{3}$. Especially, the transversion mutation $(G \rightarrow T)$ in codon 249 of the p53 tumor suppressor gene has been associated with AFB1 exposure ${ }^{4}$. A mutation in this gene may cause silencing of its tumor suppression function and allow uncontrolled cell proliferation.

Besides inducing genetic effects, AFB1 exposure also has been reported to affect the epigenome ${ }^{5-8}$.

"Epigenetic memory" has been proposed as an indicator of prior exposure 9 to for example AFB1, ultimately leading to the development of HCC. Epigenetic alterations induced by environmental stressors, such as AFB1, including changes of the normal DNA methylation pattern and microRNA expression alterations, can create a persistent memory of the received signal. It is hypothesized that each class of toxicants (with a specific "mode of action") can induce class-specific alterations in the normal pattern of DNA methylation or microRNA expression pattern (also called an "epigenetic foot-print") 9-13. These changes will thereupon induce alterations in the gene expression profile, which may promote changes in an organism's traits either immediately or at a later stage (e.g. development of HCC) 9,14.

Epigenetic changes in liver tissue from HCC patients following AFB1 exposure, have been demonstrated, e.g. gene-specific hyper-methylation (for example of RASSF1A, p16 ${ }^{7}$ and MGMT ) as well as genome-wide hypo-methylation ${ }^{8}$. This appears frequently accompanied by a mutation in the p53 gene ${ }^{4,15,16}$. In addition, specifically altered microRNA expression patterns (e.g. microRNA-221 and microRNA-122) have been observed in HCC patients 17-21.

The underlying mechanisms by which AFB1 induces DNA methylation changes and microRNA expression changes important in chemical carcinogenesis, are complex, however some potential mechanisms have been proposed. Altered microRNA expression changes occur in response to DNA damage induced by a range of environmental stressors ${ }^{14}$. Another potential mechanisms by which DNA methylation changes occur, is suggested to involve the preferential binding AFB1 to methylated $\mathrm{CpG}$ regions within the DNA, thereby inducing damage leading to conformational changes which may have an impact on the degree of methylation (e.g. causing genome-wide hypomethylation) ${ }^{6}$. Hyper- and hypo-methylation in especially the promotor region may impact on gene expression, while specifically hypomethylation may also affect genome stability. In addition, DNA damage may be generated by AFB1-induced reactive oxygen species production, which may reduce the binding affinity of methyl-CpG binding protein 2 (MECP2), thus resulting in epigenetic changes ${ }^{22}$. The MeCP2 protein binds to forms of DNA that have been methylated. The MeCP2 protein then interacts 
with other proteins to form a complex that turns off the gene. Once bound, MeCP2 will condense the chromatin structure, form a complex with histone deacetylases (HDAC), or block transcription factors directly ${ }^{23}$. The exact mechanisms by which AFB1-induced DNA methylation alterations and microRNA expression changes occur, however still remain complex and therefore need to be investigated in more detail. Furthermore, the effects of AFB1 exposure on whole genome DNA methylation and microRNA expression is not clear and therefore needs to be further explored.

However, neither in experimental studies on AFB1 carcinogenicity, nor in HCC patient studies in relation to AFB1 exposure, the persistence of epigenomic effects (including microRNA expression changes) of AFB1 exposure after removal of the treatment has yet been investigated. In addition, no attempts were made to integrate such a persistent epigenetic "footprint" with the transcriptome.

Consequently, in this present study, we hypothesize that by investigating the persistence of the AFB1-related epigenetic "footprint" together with perturbations of the transcriptome, we will be able to identify novel methyl DNA-microRNA-mRNA-interaction networks which are associated with early AFB1-induced onset of HCC. Simulation of chronic AFB1 exposure, leading to HCC, was initiated by repeated daily treatment. This is crucial since the half-life of AFB1 is 30 minutes and that of AFB1-DNA adducts in animals is less than $24 \mathrm{~h}{ }^{24,25}$. For that reason, primary human hepatocytes $(\mathrm{PHH})$ were exposed to a low dose of AFB1 for 5 days. Persistence of epigenetic effects was evaluated after 3 days of withdrawal of the carcinogen exposure. A three day wash-out period will permit the full removal of AFB1 and AFB1-DNA adducts from the $\mathrm{PHH}$, in order to prevent effects taking place due to the presence of the carcinogen or its respective DNA adducts. Whole genome DNA methylation changes were measured by means of NimbleGen 2.1 deluxe promoter arrays, microRNA expression changes were measured through Agilent microRNA microarrays while whole genome transcriptomic analysis was performed using Affymetrix whole genome gene expression microarrays. Persistent hyper- or hypo-methylated genes with a concomitant alteration in their gene expression were investigated in more detail for their biological role within AFB1-induced onset of HCC using ConsensusPathDB ${ }^{26}$ and when of relevance, by GeneCards ${ }^{27}$.

\section{Materials and methods}

\section{Cell culturing, dose determination and aflatoxin B1 treatment}

Cryopreserved primary human hepatocytes ( $\mathrm{PHH}$ ) were purchased from Life Technologies. Cells were cultured in pre-coated 24 -well plates $(700,000$ cells $/ \mathrm{ml}$ ) in a 2-layer collagen sandwich (A11428-02, Gibco), according to the supplier's protocol (Invitrogen, 2012). The following culture media were used: Hepatocyte Thawing Medium (HTM) for thawing (CM7500, Gibco), Williams' Medium E (1x, no phenol red) (A1217601, Gibco) + Cell Maintenance supplement B kit (CM4000, Gibco) for plating and incubation. After quick thawing in a water bath at $37^{\circ} \mathrm{C}$, viability of the cells was checked by a Trypan blue (CAS no. 72-57-1, Sigma-Aldrich) exclusion test as instructed in the supplier's protocol (Invitrogen, 2012). All viability scores after thawing were in agreement with those listed by the supplier. Before AFB1 treatment, cells were allowed to acclimatize for 48 hours. This is needed for the hepatocytes to restore an in vivo like cellular configuration and enzyme expression levels as optimally as possible. An AFB1 dose causing minimal (IC20) cytotoxicity after 5 days of repetitive daily exposure was established by means of the MTT assay ${ }^{28}$. Furthermore, crucial liver function enzymes such as lactate dehydrogenase $(\mathrm{LDH})$ and alanine transaminase (ALT) 
were measured. LDH and ALT were spectrophotometrically determined on a Cobas 8000 Modular Analyser (Roche Diagnostics, Basel, Switzerland). Based on this data, a dose of 0.3 $\mu \mathrm{M}$ of AFB1 (CAS no. 1162-65-8, Sigma-Aldrich), was selected for the main experiment. Control cells were exposed to $0.5 \%$ of the vehicle solvent dimethyl sulfoxide (DMSO, CAS no. 67-68-5, Sigma-Aldrich).

To diminish the possible impact of inter-donor variability, PHH from three donors of different sex, ethnicity, age, body mass index and behavioral status concerning smoking, alcohol and drug use (see Supplementary Table 7.1) were pooled. Cells were cultured in triplicate in 24 wells (RNA) or 6 wells (DNA) in a collagen sandwich layer. One part of the cells was harvested following 5 days of repetitive daily exposure to AFB1 or the vehicle control and the other part of the cells was harvested following an additional 3 day wash-out period. After all cell lysates were properly collected, total RNA was isolated.

\section{DNA methylation analyses}

\section{DNA isolation}

Cells were lysed in $500 \mu \mathrm{l}$ digestion buffer (containing $0.5 \mathrm{M}$ EDTA; $1 \mathrm{M}$ Tris-HCl, pH 8.0; $10 \%$ SDS) and incubated for 1 hour at $55^{\circ} \mathrm{C}$. Next, $25 \mu$ of proteinase $\mathrm{K}(20 \mathrm{mg} / \mathrm{ml})$ (Ambion) was added. After incubation during 1 hour at $55^{\circ} \mathrm{C}$, the proteinase $\mathrm{K}$ was inactivated for 10 minutes at $80{ }^{\circ} \mathrm{C}$. RNAse A $(2 \mu \mathrm{l} ; 100 \mathrm{mg} / \mathrm{ml})$ (Qiagen) and collagenase $(25 \mu \mathrm{l} ; 1 \%)$ (Sigma) treatment was performed for 1 hour at $37{ }^{\circ} \mathrm{C}$. Thereupon, $500 \mu \mathrm{l}$ of phenol-chloroformisoamylalcohol (PCI; 25:24:1) (Sigma) was added. The mixture was shaken manually for 5 min, and centrifuged for 5 minutes at maximum speed. The upper phase was transferred to a new Eppendorf tube and the PCI step was repeated. The upper phase was collected and precipitated using $50 \mu \mathrm{l} \mathrm{3 \textrm {M } \mathrm { NaAc }} \mathrm{pH} 5.6$ and $1250 \mu \mathrm{l}$ cold $100 \%$ ethanol for $30 \mathrm{~min}$. at $-80^{\circ} \mathrm{C}$. After centrifugation for 30 minutes at maximum speed, the DNA pellet was washed using cold $70 \% \mathrm{ETOH}$, dried in a speed vac and dissolved in $50 \mu \mathrm{l}$ nuclease free water. The total amount was at least $10 \mu \mathrm{g}$ DNA, the 260/280 ratio ranged between 1.7-1.9, and the 260/230 ratio appeared higher than 1.6. A total of 9 DNA samples were prepared.

Methylated DNA Immunoprecipitation (MeDIP), whole genome amplification (WGA) and methylation enrichment assessment

In order to obtain fragments ranging from $200 \mathrm{bp}$ to $600 \mathrm{bp}$, genomic DNA was sonicated. Next, the fragments were purified by using silica columns (Zymo Research) and eluted in TE buffer.

MeDIP was performed using the MagMeDIP kit (Diagenode, Liege, Belgium) according to the manufacturer's protocol as was described before ${ }^{29}$.

\section{MeDIP-Chip}

The Human DNA Methylation 2.1M Deluxe Promoter Array (Roche NimbleGen) was used for analyzing DNA methylation levels. Labeling and hybridization of arrays were performed according to the manufactures' protocol. Slides were washed using the NimbleGen wash buffer kit and scanned using the $2 \mu \mathrm{m}$ high resolution NimbleGen MS 200 microarray scanner.

DNA methylation data analyses and selection of differentially methylated genes (DMGs)

Signal intensity data was extracted from the scanned images of each array using NimbleScan v2.6 software and quantile normalized on a per channel basis. Log2 ratios of the intensities were computed (ratio of MeDIP signal / Input signal) and for each array, centering was 
performed by subtracting the global array bi-weight mean of the $\log 2$ ratios such that the computed $\log 2$ ratios were centered around 0 .

Detection of differential methylation was performed using the Probe Sliding WindowANOVA algorithm (PSW-ANOVA). PSW-ANOVA was implemented in the $\mathrm{R}$ statistical programming environment (v2.15.3) (http://www.r-project.org) as a custom script and was provided by Roche NimbleGen as described before ${ }^{29}$.

PSW-ANOVA (sliding window of 750 bp comprising 7 probes, and a FDR corrected p- value $<0.01$ ) was used to identify differential methylated regions (DMR) which were statistically significantly different between the different conditions tested in the experiment i.e. Exposed versus Control. Peaks were identified in the DMR by searching for regions containing at least 8 significant consecutive probes $(\mathrm{p}<0.01)$.

Peaks were mapped to promoter regions (from $3 \mathrm{~kb}$ upstream to $1 \mathrm{~kb}$ downstream of the transcription start site ${ }^{30}$ ) and $\mathrm{CpG}$ islands of genes using the NimbleScan v2.6 software. A control corrected median $\log 2$ ratio was calculated for each peak. $\log 2$ ratio's $>0$ indicate hyper methylation and $\log 2$ ratio's $<0$ indicate hypo methylation.

The DNA methylation microarray data have been deposited in NCBI's Gene Expression Omnibus (GEO) ${ }^{31}$ and are accessible through accession number GSE67005 and GSE71548.

MeDIP qPCR: validation of DNA methylation array

Standard quantitative PCR analyses were carried out for 6 genes (LNX1, DHRS7, DKK1, ENOPH1, TRIM61 and PDZD3) using $5 \mathrm{ng}$ of MeDIP and reference samples on a Bio-Rad $\mathrm{MyiQ}^{\mathrm{TM}}$. A list of primers is available in Supplementary Table 7.2. The results of the validation procedure were presented in Supplementary Figure 7.1.

\section{Gene expression analyses}

Total RNA isolation and quality control

At the end of treatment, medium was removed and PHH were harvested in Qiazol (Qiagen). Total RNA was isolated using a miRNeasy Mini Kit (Qiagen) according to the manufacturer's protocol and followed by DNase I (Qiagen) treatment. Upon purification, RNA concentrations were measured by means of a NanoDrop ${ }^{\circledR}$ ND-1000 spectrophotometer (Thermo Scientific) at 260 and $280 \mathrm{~nm}$. RNA quality and integrity were assessed by using automated gel electrophoresis on an Agilent 2100 Bioanalyzer system (Agilent Technologies). Only RNA samples which showed clear $18 \mathrm{~S}$ and $28 \mathrm{~S}$ peaks and with an RNA integrity number (RIN) higher than 8 , were used. Samples were stored at $-80^{\circ} \mathrm{C}$ until RNA hybridization.

\section{Labelling, hybridization, re-annotation}

High-density oligonucleotide GeneChips from Affymetrix were used to measure gene expression levels (Human Genome U133 Plus 2.0 array (604,258 probes)). Targets for these arrays were prepared, hybridized and scanned according to the Affymetrix protocol (Affymetrix).Normalization quality controls appeared to be within acceptable limits for almost all chips.

The mRNA microarray data have been deposited in NCBI's Gene Expression Omnibus (GEO) ${ }^{31}$ and are accessible through accession number GSE67002 and GSE71547. 


\section{$\underline{\text { Re-annotation, normalization and data filtering }}$}

The Arrayanalysis.org web service was used for quality control ${ }^{32}$ and all microarrays except one were of high quality which was omitted from the analyses. CEL files were imported into $\mathrm{R}$ v2.15.3 (http://www.r-project.org) using the "affy" library 33 within BioConductor (v2.9) 34 . Probe re-annotation, normalization and data filtering was performed as previously described ${ }^{35}$.

\section{Selection of differentially expressed genes (DEGs)}

Differentially expressed genes (DEGs) were selected using the linear model for microarrays (LIMMA) approach ${ }^{36}$. The resulting p-values were FWER-corrected using the False Discovery Rate (FDR) method. The following criteria were applied: (1) average expression in at least one of the experimental groups $>6$ (log2-scale), (2) a FDR-corrected p-value $<0.05$ obtained through a moderated t-test, and (3) for the three replicates an average absolute fold change (FC) of 1.5 or higher (i.e., average $\log 2$ ratio of $<-0.58$ or $>0.58$ ).

\section{MicroRNA expression analyses}

MicroRNA expression profiling was executed by means of Agilent Sureprint G3 Unrestricted Human miRNA V19 $8 \times 60 \mathrm{~K}$ microarrays. The hybridization was done according to standard protocols; next, the microarray slides were washed and scanned by means of a DNA microarray scanner (Agilent Technologies). The scanned images were converted into TXT files by means of the Feature Extraction Software v10.7.3.1 from Agilent Technologies, which were imported into R 2.15.3 (http://www.r-project.org) for quality control via an in-house developed pipeline ${ }^{37}$. Filtering and normalization was done using AgiMicroRna ${ }^{37,38}$. Total gene signals were $\log 2$-transformed and quantile-normalized. The selection criteria for identifying differentially expressed microRNAs (DE-miRs) were the same as those for DEGs. The DE-miRs between the 5-day exposure period and the 3-day wash-out period were then compared with the purpose of identifying persistently expressed DE-miRs, transiently expressed and exclusively expressed after washout.

The microRNA microarray data have been deposited in NCBI's Gene Expression Omnibus (GEO) ${ }^{31}$ and are accessible through accession number GSE67003 and GSE71545.

\section{Identification of persistent and HCC-related DMG and DEGs}

No persistently affected DE-miRs were found after the 3-day wash-out period. Therefore no microRNAs were included in the follow-up analyses. For the selection of genes of interest for further analysis, a 4-step approach was followed (see Figure 7.1). In the first step, a comparison was made (based on their direction of change) between the DMGs found after 5 days of exposure to AFB1 and the DMGs found after 3 days of wash-out period. A list of persistent differentially hyper-methylated (386 DMGs) and hypo-methylated (1134 DMGs) genes was generated (see Venn diagrams in Step 1 of Figure 7.1). 


\section{Step-by-step approach to identify persistent and HCC-related DEGs and DMGs}

and upregulated after the 5-day exposure and the 3-day washout period

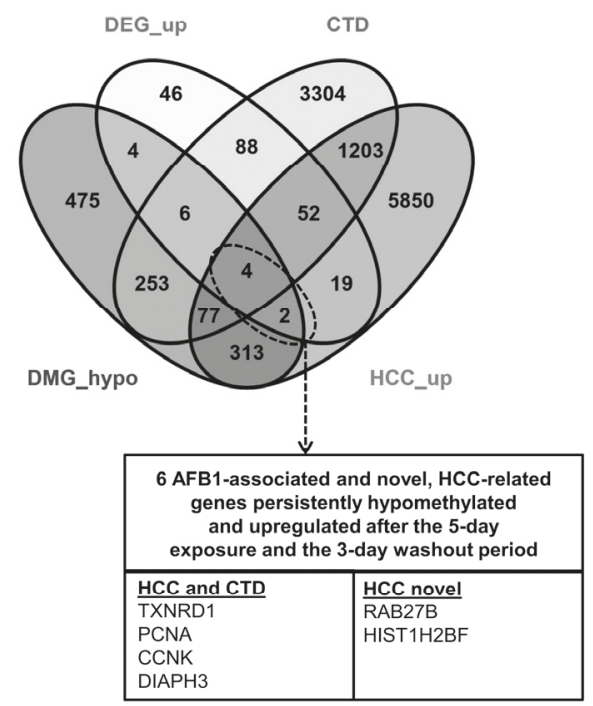

Figure 7.1: A step-by-step approach was followed in order to identify persistently expressed hyper- or hypomethylated genes which are also affected in their gene expression (down or up-regulation respectively) after 5-day exposure to AFB1 and a 3-day of wash-out period. In step 1 persistently hyper resp. hypo-methylated genes were selected. In step 2 hyper resp. hypo-methylated genes were selected that showed a down resp. up-regulation in their expression after 5 days of exposure to AFB1 and after 3 days of wash-out. Then they were compared with an HCC profile and a list of AFB1-associated genes. In step 5 the AFB1-associated and novel, HCC-related hyper-methylated down-regulated and hypo-methylated - up-regulated genes were identified.

In the second step, persistently expressed DMGs were mapped to their gene expression changes. Only those genes were selected that were differentially expressed after the 5 day AFB1 exposure-period and after the 3-day wash-out period (see $\underline{\text { Step } 2}$ of Figure 7.1). After methylation data were matched with gene expression data, a selection of transcripts was made based on previously obtained information concerning: their relation with HCC resp. their association with AFB1 exposure (see $\underline{\text { Step } 3}$ of Figure 7.1). The first selection of transcripts was based on the comparison with a HCC transcriptome signature (based on HCC versus healthy human liver) (created by Caiment et al. in 2014) ${ }^{39}$ by exploiting publically available gene expression data sets from ArrayExpress (HCC versus healthy human liver) (see part K of Supplementary Table 7.3). A second comparison was made with a list of genes which have previously been associated with AFB1 exposure (obtained by curated data found in the 
Comparative Toxicogenomics Database (CTD) 40 (http://www.ctdbase.org/) (see Venn diagrams in Step 3 of Figure 7.1) (see part J of Supplementary Table 7.3). In the last step, all AFB1-associated and novel, HCC-related hyper-methylated - down-regulated and hypomethylated - up-regulated genes were identified (see Step 4 of Figure 7.1).

\section{$\underline{\text { Results }}$}

\section{Determination of differentially methylated genes (DMGs)}

After a pool of $\mathrm{PHH}$ was repetitively exposed for 5 days to $0.3 \mu \mathrm{M}$ of AFB1, 5639 genes were found to be differentially methylated (1896 genes were hyper-methylated and 3743 genes were hypo-methylated) (see part A and B of Supplementary Table 7.3). After 5 days of exposure, the carcinogen was removed from the medium, and after 3 days of wash out, 8131 differentially methylated genes were identified (4397 genes were significantly hyper-methylated and 3734 genes were hypo-methylated) (see part C and D of Supplementary Table 7.3). From the 5639 DMGs identified at day 5 of AFB1 exposure, 386 genes remained hyper-methylated and 1134 genes remained hypo-methylated after the 3-day wash-out period (see part E and $\mathrm{F}$ of Supplementary Table 7.3).

\section{Identification of differentially expressed genes (DEGs)}

1490 genes (702 up-regulated and 788 down-regulated) (see part G of Supplementary Table 7.3) were differentially expressed in the pool of $\mathrm{PHH}$ after 5 days of repetitive exposure to 0.3 $\mu \mathrm{M}$ of AFB1. When the treatment was terminated, wash out for 3 days resulted in 896 DEGs (368 up-regulated and 528 down-regulated) (see part H of Supplementary Table 7.3). After the 3-day wash-out period, 598 genes (221 up-regulated and 377 down-regulated) appeared persistently expressed into the same direction (see part I of Supplementary Table 7.3).

892 genes (481 up-regulated and 411 down-regulated) appeared only expressed after the 5-day exposure period, while 298 genes (147 up-regulated and 151 down-regulated) were activated only during the wash-out (see part I of Supplementary Table 7.3).

\section{Selection of differentially expressed microRNAs (DE-miRs)}

No microRNAs were differentially expressed after 5 days of AFB1 treatment in the PHH pool. After the exposure period was ended two microRNAs appeared differentially expressed after the 3-day wash-out period; hsa-miR-513b and hsa-miR-1275 (see part L of Supplementary Table 7.3). Since these two microRNAs were not expressed after the 5-day exposure period, no persistently expressed microRNAs could be identified following exposure to $0.3 \mu \mathrm{M}$ of AFB1.

\section{Identification of persistently expressed DMGs and DEGs}

The expression of 2 of the 386 persistently hyper-methylated genes was significantly downregulated (see part A of Table 7.1).

None of the two hyper-methylated genes which were significantly affected in their expression after the 5-day exposure period as well as after the 3-day wash-out period, could be linked with the HCC-profile or with the AFB1-associated genes (see part A of Table 7.1).

2 persistently hypo-methylated genes which demonstrated a significant up regulation in their expression after the 5-day exposure period as well as after the 3 -day wash-out period, could be associated with an HCC expression profile and were not associated with AFB1 exposure before while 4 of these HCC-related hypo-methylated genes, with a significant up regulation in their expression after the 5-day exposure period as well as the 3-day wash-out period, are already known to respond to AFB1 exposure (see part B of Table 7.1). 
16 of the persistently hypo-methylated genes were also significantly up-regulated after both the 5-day exposure period as well as the 3-day wash-out period (16 genes) (see part B of Table 7.1).

Table 7.1: List of, A) 2 persistently hypermethylated - downregulated genes and B) 16 persistently hypomethylated upregulated genes, following 5 days of exposure to $0.3 \mu \mathrm{M}$ of AFB1 and 3 days of wash-out (HCC relation is based on profile by Caiment et al., 2014, AFB1 association is retrieved from Comparative Toxicogenomics Database (CTD)).

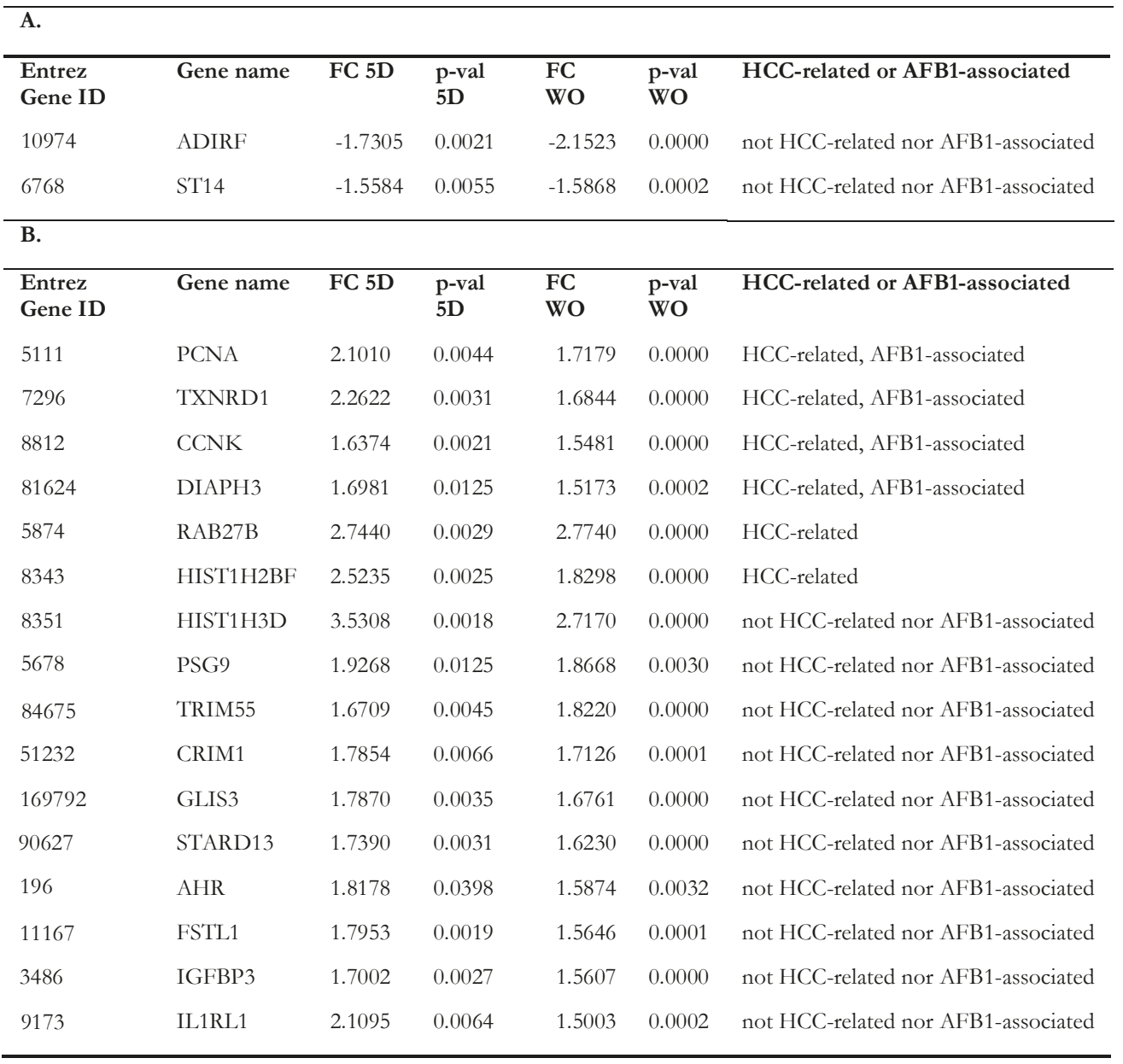

\section{Discussion}

In this study, a pool of PHH was exposed to $0.3 \mu \mathrm{M}$ of AFB1 for 5 repetitive days. In addition, a three day wash-out period was inserted which permitted the full removal of AFB1 and AFB1-DNA adducts from the PHH, in order to avoid effects occurring due to the presence of the carcinogen or its respective DNA adducts. We were able to identify persistent effects on the epigenome as well as on the transcriptome level. No persistent effects could be observed in the microRNAome. Approximately 20\% (386 out of 1896) of the hyper methylated genes after the 5-day AFB1 exposure period, remained hyper-methylated during wash out (see part E of Supplementary Table 7.3). Roughly 30\% (1134 out of 3743) of the AFB1-induced hypomethylated genes retained their epigenetically altered status after withdrawal of the carcinogen 
exposure (see part F of Supplementary Table 7.3). In general, more genes appeared persistently hypo-methylated than hyper-methylated which is of interest in view of the fact that in HCC patients liver tissues in association with AFB exposure, hypo-methylation prevails ${ }^{7,8}$.

Nearly 47\% (377 out of 788) of the down-regulated DEGs, after the 5-day exposure period, remained down-regulated during wash out. Approximately 31\% (221 out of 702) of the upregulated DEGs preserved their epigenetically changed status after termination of the carcinogen exposure.

$0.5 \%$ (2 out of 386) (see part A of Table 7.1) of the persistently hyper-methylated genes and $1.4 \%$ (16 out of 1134) (see part B of Table 7.1) of the persistent hypo-methylated genes were also affected on the transcriptome level after wash out. By using a HCC-signature gene expression profile, based on a comparison of HCC tissue with healthy liver tissue as obtained by Caiment and colleagues ${ }^{41}, 6$ of the persistently affected hypo-methylated and downregulated genes could be associated with HCC. Four of these genes were previously known to be inducible by AFB1 exposure, as reported by $\mathrm{CTD}^{40}$. The persistent epigenetic effect of AFB1 on these genes after withdrawal of the treatment has not been described before and thus presents a novel finding.

Gene-functions of the four AFB1-responsive, HCC-related hypo-methylated and up-regulated genes (i.e. TXNRD1, PCNA, CCNK and DIAPH3) and the two novel HCC-associated hypomethylated and up-regulated genes (i.e. RAB27A and HIST1H2BF) were studied in more detail to further investigate their importance in AFB1-induced liver carcinogenesis.

Thioredoxin Reductase 1 (TXNRD1 - 7296) encodes a member of the family of pyridine nucleotide oxidoreductases. The TXNRD1 encoded protein reduces thioredoxins in addition to other substrates by using NADPH within the thioredoxin redox system. Furthermore, it plays a role in selenium metabolism and offers protection against oxidative stress. TXNRD1 is a selenoprotein, therefore each subunit of the protein contains a selenocysteine $(\mathrm{Sec})$ residue which is required for catalytic activity ${ }^{27}$. Several studies have implicated that overexpression of, especially reduced thioredoxin, is one of the enhancers of cancer cell growth, either through the direct stimulation of cancer cell growth or through the inhibition of cancer cell apoptosis 42. Remarkably, it has been shown that selenium deficiency, leading to down-regulation of TXNRD1, protects against the hepatocarcinogenic effects of AFB1 in rats ${ }^{43}$. This might indicate that when TXNRD1, which requires selenium, is increased, the effects will be opposite indicating a more severe effect of AFB1 on the liver. The mechanism behind this finding is that TXNRD1 activates transcriptional activity of NFkappaB and AP-1, causing changes in many biological pathways such as cell proliferation 43,44 . Furthermore, selenium deficiency or down-regulation of TXNRD1 increases the expression of AFB1-aldehyde reductase (AFAR) and and glutathione-S-transferase (GST) ${ }^{43}$. These two phase II enzymes are responsible for the detoxification of AFB1-8,9-epoxide via glutathione conjugation into less active metabolites, thereby preventing the formation of AFB1-adducts with macromolecules ${ }^{43}$. An increased expression of TXNRD1 might have an opposite effect thereby decreasing AFAR and GST level leading to an increased formation of AFB1-adducts within the liver. These results were based on rat studies and therefore need to be validated in humans. Nevertheless, the finding that persistently hypo-methylated and up-regulated TNXRD1 is related with the regulation of important AFB1detoxifying enzymes, AFAR and GST, and affects the activity of NFkappaB and AP-1, might help in better understanding the mechanisms underlying the induction of HCC by AFB1.

Proliferating cell nuclear antigen (PCNA - 5111) codes for a cofactor of DNA polymerases that encircles DNA (forming a DNA sliding clamp) thereby orchestrating several important 
processes by the recruitment of crucial players to the replication fork. PCNA needs to be ubiquitinated in order to function; once activated, it acts as a scaffold to recruit proteins involved in DNA replication (including error-prone bypass replication or translesion synthesis (TLS)), DNA repair (e.g. mismatch repair (MMR), cell cycle, apoptosis, chromatin remodeling and preservation of epigenetic marks ${ }^{45}$. One epigenetic mechanism controlled by PCNA is the methylation at the $\mathrm{C} 5$ position of cytosine residues, predominantly found in $\mathrm{CpG}$ sequence. DNA cytosine methyltransferase 1 (DNMT1) is thereby recruited by PCNA to sites of DNA repair, presumably to restore the DNA methylation status after repair DNA synthesis took place ${ }^{45}$. Main regulators of the chromatin state are histone acetyltransferases (HATs) and histone deacetylation enzymes (HDACs), and some of these enzymes also are linked to PCNA (e.g. HDAC1 and p300) ${ }^{45}$. In HCC patients, overexpression of PCNA is associated with HCC de-differentiation, shorter survival and higher recurrence rates ${ }^{46}$. Another study also indicates that apoptosis is dysregulated and proliferation activity perturbed due to PCNA overexpression 47,48. The role of PCNA in many cellular processes, together with the fact that there is a clear association with PCNA overexpression and dysregulation of apoptosis and proliferation activity in HCC, indicates that the persistent hypo-methylation and up-regulation measured within this study, may thus be an important finding for understanding the early development of AFB1-induced HCC.

Cyclin K (CCNK - 8812), remained persistently hypo-methylated and up-regulated after the 3day washout period, is a member of the transcription cyclin family ${ }^{27}$. These types of cyclins may regulate transcription through their association with, and activation of, cyclin-dependent kinases (CDK) that phosphorylate the C-terminal domain (CTD) of the large subunit of RNA polymerase II (POLR2A) 49-51. Binding of CCNK with CDK12, forming the complex $\mathrm{CCNK} / \mathrm{CDK} 12$, regulate the expression of several DNA damage response genes, including some critical regulators of genomic stability, such as BRCA1, ATR, FANCI and FANCD2 ${ }^{52}$. An oncogenic function of CCNK has been implicated in myeloma cells, where overexpression leads to several important affected biological processes such as cell metabolism, signal transduction, regulation of transcription and translation ${ }^{53}$. Although overexpression of CCNK has not been reported in HCC, considering its role in the regulation of several DNA damage responsive genes and its oncogenic effect in myeloma, it might have a crucial role in early onset of AFB1-associated HCC as well.

The AFB1-induced and HCC-related diaphanous-Related Formin 3 (DIAPH3 - 81624) gene, also persistently hypo-methylated and up-regulated, encodes a member of the diaphanous subfamily of the formin family ${ }^{27}$. Formins are the largest group of directly regulated RhoGTPase effectors which bind to the GTP-bound form of Rho and to profilin. The Rho family of GTPases is a subfamily of the Ras protein superfamily of small GTPases ${ }^{54}$. Recruitment of profilin to the membrane, promotes actin polymerization and remodeling and the regulation of cell movement and adhesion. This is required for cytokinesis and stress fiber formation 27. Overexpression of members of the formin family have been associated with metastasis, specifically invasion in surrounding tissues, of cancer cells ${ }^{55}$. Considering the role of formin proteins, like DIAPH3, in actin remodeling, cell movement and metastasis in cancer, its persistent expression might also be important in the development of HCC initiated by AFB1 exposure.

A novel HCC-related gene Ras-related protein Rab-27B (RAB27B - 5874), observed to be persistently hypo-methylated and up-regulated, is part of the Rab GTPase family which normally responds to cell signaling and metabolic demands through the coordinated regulation of membrane trafficking ${ }^{56}$. The Rab GTPase subfamily is, next to the Rho GTPases, part of the Ras protein superfamily of small GTPases ${ }^{54}$. Rab family proteins are important in 
regulating signal transduction and cellular processes such as differentiation, proliferation, vesicle transport, nuclear assembly, and cytoskeleton formation. In addition, it has been observed that membrane transport to the cell surface is differentially regulated during specific stages of the cell cycle ${ }^{57}$. From certain members of the Rab family (e.g. RAB25A) it has been clearly shown that they decrease apoptosis, as well as to increase both the proliferation and aggressiveness of cancers ${ }^{58}$. In a study within HCC patients they found that RAB27B expression was closely correlated with tumor progression as well ${ }^{59}$. Hypo-methylation of RAB27A in HCC has however not been reported yet. Our results suggest that persistent, epigenome-mediated, expression of RAB27A considering its role in various processes e.g. cell growth and differentiation, might therefore be of relevance for explaining the carcinogenic mode-of-action of AFB1.

Lastly, the novel HCC-associated Histone Cluster 1, H2bf (HIST1H2BF - 8343) gene, persistently hypo-methylated and up-regulated after the 3-day washout period, encodes a member of the histone H2B family. Histones package and order the DNA into nucleosome structures within the nucleus of eukaryotes. Next to the core histones (histones H2A, H2B, H3 and H4) linker histones (H1 and H5) exist ${ }^{60}$. The linker histones make sure that the DNA, which is wrapped around octameric nucleosomes (consisting of core histones), is kept into place. Post-translational modifications of histones play a critical role in gene expression control. Especially the deregulation of histone $\mathrm{H} 2 \mathrm{~B}$ variants by increased ubiquitination and decreased methylation have been implicated to contribute to cancer development ${ }^{61-63}$. Hypomethylation of HIST1H2BF has not been reported yet in AFB1-associated development of HCC and could therefore represent a novel finding in order to better understand the underlying mechanisms.

\section{Conclusion}

In conclusion, we have identified persistent effects of AFB1 exposure on the epigenome and the transcriptome. By performing an integrative analysis, we were able to identify six important gene candidates, demonstrating a specific AFB1-related "epigenetic foot-print" as indicated by their persistent expression and DNA methylation change after the carcinogen exposure was ended. These six genes play a role in several biological processes which are known to be affected by AFB1 exposure and altered in HCC e.g. increase of AFB1-DNA adducts formation, dysregulation of the DNA damage response and changes in cell growth and adhesion (via Rho and Rab GTPase signaling) leading to metastasis of cancer cells.

These findings need to be further validated on the proteome level to observe the actual effect of these epigenetic changes on protein expression. In addition, post-translational modifications of these proteins should be investigated since many of the proteins encoded by the discussed genes (e.g. PCNA) need modification in order to be activated. Furthermore, a longer wash-out period could be applied in order to observe whether the effects remain persistently altered. Nevertheless, this study is the first to report on the persistence of epigenetic and transcriptomic effects after withdrawal of carcinogen treatment. Therefore, these findings may, on the long run, be useful for supporting human cancer risk assessment.

\section{Supplementary data}

Supplementary Figures 7.1 and Tables 7.1-7.3 are available upon request. 


\section{References}

1. Liu, Y. \& Wu, F. Global burden of aflatoxin-induced hepatocellular carcinoma: a risk assessment. Environ Health Perspect 118, 818-24 (2010).

2. Wild, C.P. \& Gong, Y.Y. Mycotoxins and human disease: a largely ignored global health issue. Carcinogenesis 31, 71-82 (2010).

3. Groopman, J.D., Kensler, T.W. \& Wild, C.P. Protective interventions to prevent aflatoxin-induced carcinogenesis in developing countries. Annu Rev Public Health 29, 187-203 (2008).

4. Soini, Y. et al. An aflatoxin-associated mutational hotspot at codon 249 in the p53 tumor suppressor gene occurs in hepatocellular carcinomas from Mexico. Carcinogenesis 17, 1007-12 (1996).

5. Zhang, Y.J. et al. High frequency of promoter hypermethylation of RASSF1A and p16 and its relationship to aflatoxin B1-DNA adduct levels in human hepatocellular carcinoma. Mol Carcinog 35, 85-92 (2002).

6. Zhang, Y.J. et al. Inactivation of the DNA repair gene O6-methylguanine-DNA methyltransferase by promoter hypermethylation and its relationship to aflatoxin B1-DNA adducts and p53 mutation in hepatocellular carcinoma. Int J Cancer 103, 440-4 (2003).

7. Zhang, Y.J. et al. Aflatoxin B1 and polycyclic aromatic hydrocarbon adducts, p53 mutations and p16 methylation in liver tissue and plasma of hepatocellular carcinoma patients. Int J Cancer 119, 985-91 (2006).

8. Zhang, Y.J. et al. Global hypomethylation in hepatocellular carcinoma and its relationship to aflatoxin B(1) exposure. World J Hepatol 4, 169-75 (2012).

9. Mirbahai, L. \& Chipman, J.K. Epigenetic memory of environmental organisms: a reflection of lifetime stressor exposures. Mutat Res Genet Toxicol Environ Mutagen 764-765, 10-7 (2014).

10. Goodman, J.I. et al. What do we need to know prior to thinking about incorporating an epigenetic evaluation into safety assessments? Toxicol Sci 116, 375-81 (2010).

11. Moggs, J.G., Goodman, J.I., Trosko, J.E. \& Roberts, R.A. Epigenetics and cancer: implications for drug discovery and safety assessment. Toxicol Appl Pharmacol 196, 422-30 (2004).

12. Legler, J. Epigenetics: an emerging field in environmental toxicology. Integr Environ Assess Manag 6, 314-5 (2010).

13. Koufaris, C., Wright, J., Currie, R.A. \& Gooderham, N.J. Hepatic microRNA profiles offer predictive and mechanistic insights after exposure to genotoxic and epigenetic hepatocarcinogens. Toxicol Sci 128, 532-43 (2012).

14. Izzotti, A. \& Pulliero, A. The effects of environmental chemical carcinogens on the microRNA machinery. International Journal of Hygiene and Environmental Health 217, 601-627 (2014).

15. Hsu, I.C. et al. Mutational hotspot in the p53 gene in human hepatocellular carcinomas. Nature 350, 427-8 (1991).

16. Hussain, S.P., Schwank, J., Staib, F., Wang, X.W. \& Harris, C.C. TP53 mutations and hepatocellular carcinoma: insights into the etiology and pathogenesis of liver cancer. Oncogene 26, 2166-76 (2007).

17. Fornari, F. et al. MiR-221 controls CDKN1C/p57 and CDKN1B/p27 expression in human hepatocellular carcinoma. Oncogene 27, 5651-61 (2008).

18. Fornari, F. et al. MiR-122/cyclin G1 interaction modulates p53 activity and affects doxorubicin sensitivity of human hepatocarcinoma cells. Cancer Res 69, 5761-7 (2009).

19. Gramantieri, L. et al. MicroRNA involvement in hepatocellular carcinoma. J Cell Mol Med 12, 2189-204 (2008).

20. Karakatsanis, A. et al. Expression of microRNAs, miR-21, miR-31, miR-122, miR-145, miR-146a, miR200c, miR-221, miR-222, and miR-223 in patients with hepatocellular carcinoma or intrahepatic cholangiocarcinoma and its prognostic significance. Mol Carcinog 52, 297-303 (2013).

21. Sun, J., Lu, H., Wang, X. \& Jin, H. MicroRNAs in hepatocellular carcinoma: regulation, function, and clinical implications. ScientificW orldJournal 2013, 924206 (2013).

22. Valinluck, V. et al. Oxidative damage to methyl-CpG sequences inhibits the binding of the methyl-CpG binding domain (MBD) of methyl-CpG binding protein 2 (MeCP2). Nucleic Acids Res 32, 4100-8 (2004).

23. Yasui, D.H. et al. Integrated epigenomic analyses of neuronal MeCP2 reveal a role for long-range interaction with active genes. Proc Natl Acad Sci US A 104, 19416-21 (2007).

24. Qian, G. et al. Physiologically based toxicokinetics of serum aflatoxin B1-lysine adduct in F344 rats. Toxicology 303, 147-51 (2013).

25. Lai, H. et al. Association between aflatoxin B1 occupational airway exposure and risk of hepatocellular carcinoma: a case-control study. Tumour Biol 35, 9577-84 (2014).

26. Kamburov, A., Wierling, C., Lehrach, H. \& Herwig, R. ConsensusPathDB--a database for integrating human functional interaction networks. Nucleic Acids Res 37, D623-8 (2009).

27. Rebhan, M., Chalifa-Caspi, V., Prilusky, J. \& Lancet, D. GeneCards: integrating information about genes, proteins and diseases. Trends Genet 13, 163 (1997).

28. Mosmann, T. Rapid colorimetric assay for cellular growth and survival: application to proliferation and cytotoxicity assays. J Immunol Methods 65, 55-63 (1983). 
29.

30.

31.

32.

33.

34.

35.

36.

37.

38.

39.

40.

41.

van Breda, S.G. et al. Epigenetic mechanisms underlying arsenic-associated lung carcinogenesis. Arch Toxicol (2014).

Young, M.D. et al. ChIP-seq analysis reveals distinct H3K27me3 profiles that correlate with transcriptional activity. Nucleic Acids Res 39, 7415-27 (2011).

Edgar, R., Domrachev, M. \& Lash, A.E. Gene Expression Omnibus: NCBI gene expression and hybridization array data repository. Nucleic Acids Res 30, 207-10 (2002).

Eijssen, L.M. et al. User-friendly solutions for microarray quality control and pre-processing on ArrayAnalysis.org. Nucleic Acids Res 41, W71-6 (2013).

Gautier, L., Cope, L., Bolstad, B.M. \& Irizarry, R.A. affy--analysis of Affymetrix GeneChip data at the probe level. Bioinformatics 20, 307-15 (2004).

Gentleman, R.C. et al. Bioconductor: open software development for computational biology and bioinformatics. Genome Biol 5, R80 (2004).

Rieswijk, L., Lizarraga, D., Brauers, K.J., Kleinjans, J.C. \& van Delft, J.H. Characterisation of cisplatininduced transcriptomics responses in primary mouse hepatocytes, HepG2 cells and mouse embryonic stem cells shows conservation of regulating transcription factor networks. Mutagenesis 29, 17-26 (2014).

Smyth, G.K. Limma: linear models for microarray data. , 397-420 (Springer, New York, 2005).

Coonen, M., Theunissen, D.H., Kleinjans, J.C. \& Jennen, D.G. MagiCMicroRna: a web implementation of AgiMicroRna using shiny. Source Code Biol Med 10, 4 (2015).

Lopez-Romero, P. Pre-processing and differential expression analysis of Agilent microRNA arrays using the AgiMicroRna Bioconductor library. BMC Genomics 12, 64 (2011).

Caiment, F., Tsamou, M., Jennen, D. \& Kleinjans, J. Assessing compound carcinogenicity in vitro using connectivity mapping. Carcinogenesis 35, 201-7 (2014).

Davis, A.P. et al. The Comparative Toxicogenomics Database: update 2013. Nucleic Acids Res 41, D1104-14 (2013).

Caiment, F., Tsamou, M., Jennen, D. \& Kleinjans, J. Assessing compound carcinogenicity in vitro using connectivity mapping. Carcinogenesis Advance Access published August 12, 2013, doi:10.1093/carcin/bgt278 (2013).

Saitoh, M. et al. Mammalian thioredoxin is a direct inhibitor of apoptosis signal-regulating kinase (ASK) 1. EMBO J 17, 2596-606 (1998).

McLeod, R. et al. Protection conferred by selenium deficiency against aflatoxin B1 in the rat is associated with the hepatic expression of an aldo-keto reductase and a glutathione S-transferase subunit that metabolize the mycotoxin. Cancer Res 57, 4257-66 (1997).

Morgan, M.J. \& Liu, Z.-g. Crosstalk of reactive oxygen species and NF-xB signaling. Cell Research 21, $103-$ 115 (2011).

Moldovan, G.L., Pfander, B. \& Jentsch, S. PCNA, the maestro of the replication fork. Cell 129, 665-79 (2007).

Hu, T.H. et al. Down-regulation of tumor suppressor gene PTEN, overexpression of p53, plus high proliferating cell nuclear antigen index predict poor patient outcome of hepatocellular carcinoma after resection. Oncol Rep 18, 1417-26 (2007).

Qin, L.F., Ng, I.O., Fan, S.T. \& Ng, M. p21/WAF1, p53 and PCNA expression and p53 mutation status in hepatocellular carcinoma. Int J Cancer 79, 424-8 (1998).

Alenzi, F.Q. et al. Original Article: Investigation of Bcl-2 and PCNA in Hepatocellular Carcinoma: Relation to Chronic HCV. J Egypt Natl Canc Inst 22, 87-94 (2010).

Fu, T.J., Peng, J., Lee, G., Price, D.H. \& Flores, O. Cyclin K functions as a CDK9 regulatory subunit and participates in RNA polymerase II transcription. J Biol Chem 274, 34527-30 (1999).

Edwards, M.C. et al. Human CPR (cell cycle progression restoration) genes impart a Far- phenotype on yeast cells. Genetics 147, 1063-76 (1997).

Edwards, M.C., Wong, C. \& Elledge, S.J. Human cyclin K, a novel RNA polymerase II-associated cyclin possessing both carboxy-terminal domain kinase and Cdk-activating kinase activity. Mol Cell Biol 18, 4291300 (1998).

Blazek, D. et al. The Cyclin K/Cdk12 complex maintains genomic stability via regulation of expression of DNA damage response genes. Genes Dev 25, 2158-72 (2011).

Marsaud, V. et al. Cyclin K and cyclin D1b are oncogenic in myeloma cells. Mol Cancer 9, 103 (2010).

Wennerberg, K., Rossman, K.L. \& Der, C.J. The Ras superfamily at a glance. J Cell Sci 118, 843-6 (2005).

Nürnberg, A., Kitzing, T. \& Grosse, R. Nucleating actin for invasion. Nat Rev Cancer 11, 177-187 (2011).

Agola, J.O., Jim, P.A., Ward, H.H., Basuray, S. \& Wandinger-Ness, A. Rab GTPases as regulators of endocytosis, targets of disease and therapeutic opportunities. Clin Genet 80, 305-18 (2011).

Brennwald, P. Membrane traffic: the exocyst meets the cell cycle. Curr Biol 23, R838-40 (2013).

Subramani, D. \& Alahari, S.K. Integrin-mediated function of Rab GTPases in cancer progression. Mol Cancer 9, 312 (2010).

Dong, W.W. et al. Differential expression of Rab27A/B correlates with clinical outcome in hepatocellular carcinoma. World J Gastroenterol 18, 1806-13 (2012). 
60. Hartl, D.L., Freifelder, D. \& Snyder, L.A. Basic genetics, xvi, 505 p. (Jones and Bartlett Publishers, Boston, 1988).

61. Nayak, S.R. et al. A Role for Histone H2B Variants in Endocrine-Resistant Breast Cancer. Horm Cancer (2015).

62. Espinosa, J.M. Histone H2B ubiquitination: the cancer connection. Genes Dev 22, 2743-9 (2008).

63. Hou, L., Zhang, X., Wang, D. \& Baccarelli, A. Environmental chemical exposures and human epigenetics. Int J Epidemiol 41, 79-105 (2012). 
C

0

○

$\bigcirc$

o Chapter 8

O Summary and Genefal discussion

00
0

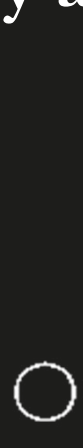

00

O

O

O

O

O

00

30

0

C

0

0

C

(C) 0

00

00

(3)

00

O

0

5

O

0 


\section{Summary and General discussion}

Cancer is the most common cause of disease-related mortality worldwide and is expected to increase dramatically within the coming 20 years ${ }^{1}$. Liver cancer, specifically hepatocellular carcinoma (HCC), is the sixth most diagnosed type of cancer and the second most lethal kind of cancer globally ${ }^{1}$. Particularly in the less developed parts of the world this seems to be an enormous health problem due to, besides genetic elements, mainly biological (i.e. hepatitis B or $\mathrm{C}$ viruses) and environmental factors (i.e. aflatoxin B1 exposure) ${ }^{2}$. Around one third of all new cases of HCC may be prevented by proper control and avoidance of environmental carcinogen exposure (i.e. chemicals, drugs and nutrients) ${ }^{1}$.

Traditionally environmental carcinogens may be assigned to two different classes based on their mode of action (MOA); genotoxic (GTX) and non-genotoxic (NGTX) carcinogens ${ }^{3}$. GTX carcinogens (GTXC) directly react with the DNA, thereby initiating mutations leading to cancer-related events. NGTX carcinogens (NGTXC) promote cancer via diverse non-DNA reactive mechanisms e.g. induction of oxidative stress via binding to the arylhydrocarbon receptor $(\mathrm{AhR})$ or the peroxisome proliferator-activated receptor alpha (PPAR- $\alpha)^{4}$. In order to properly control and avoid exposure to environmental carcinogens, accurate and sensitive chemical risk assessment of genotoxicity and carcinogenicity is required; to avoid hazardous agents from entering the public market, limit the carcinogen exposure in humans and reduce the number of new cases of HCC.

New chemicals, drugs or nutrients are conventionally tested within a battery of in vitro genotoxicity tests which is used as a predictor of their long-term carcinogenic potential5-9. Once a chemical, drug or nutrient has been found positive based on these in vitro tests, additional in vivo tests are required. The current in vitro genotoxicity tests are however characterized with a high false-positive rate $^{10}$ and, more importantly, non-genotoxic carcinogens remain undetected ${ }^{11}$. In vivo validation of positive results from the in vitro genotoxicity assays is standardly performed by the 2 -year lifetime rodent bioassay. These tests are time and money consuming and require the lives of, often, many unnecessary animals. Another disadvantage of the lifetime rodent bioassay is the fact that they are fairly limited in their capacity to predict in vivo carcinogenicity in humans $(\sim 50 \%)^{12}$. In vivo assays therefore, in addition to the in vitro tests, generate a high degree of false positives (specific rodent carcinogens) and false negatives (exclusive human carcinogens) ${ }^{12}$. Due to a lack of more accurate and sensitive assays to evaluate genotoxic and (non-genotoxic) carcinogenic potential of chemicals within human risk assessment, there is a high demand for more reliable alternative systems and assays ${ }^{13}$.

The focus of human risk assessment has therefore shifted towards a more systems biology approach ${ }^{14-16}$. The rationale behind this new strategy is that by obtaining a better understanding of the biological mechanisms underlying genotoxicity and carcinogenicity, a more accurate and sensitive prediction may be provided for in vivo human risk assessment ${ }^{17}$. The field of toxicogenomics, in which 'omics-based high-throughput technologies are applied seems to be a promising area for developing such alternative systems ${ }^{18}$. Mainly by the integration of several layers within the biological system, more insights may be obtained which aid in better understanding of the underlying carcinogenic mechanisms ${ }^{19}$. Processes regulating transcription (i.e. DNA methylation) and translation (i.e. regulation by short non-coding microRNAs) should be taken into account here as well ${ }^{20-22}$. Furthermore, the identification of important regulatory genes and processes acting within particular toxic or adverse outcome pathways (AOPs) ${ }^{17}$ might be relevant for prediction purposes within human risk assessment ${ }^{20-22}$. Many of these regulatory mechanisms seem to be affected in the diseased state, for example in HCC. 
Some microRNAs with a tumor-suppressive function are downregulated within HCC ${ }^{23-29}$. Likewise, in HCC, aberrant promotor DNA hypermethylation of specific tumor suppressor genes and global genomic DNA hypomethylation are observed, and have been widely studied ${ }^{30-37}$. The interplay between these different regulatory layers seems to affect the transcriptome, thereby determining the fate of the cell ${ }^{38}$. Chemical carcinogen exposure also seems to affect this regulatory network and may even, if the effects are persistent, be predictive for the early signs of the disease, such as $\mathrm{HCC}^{21}$. The aim of this thesis was therefore to further unravel the role of microRNAs and epigenetic changes in transcriptomic responses in these regulatory networks upon exposure to GTX and NGTX compounds in the in vitro liver.

\section{Identification of a robust in vitro liver model for genotoxicity and carcinogenicity testing}

In order to develop a more accurate in vitro alternative system for genotoxicity and carcinogenicity testing, it is important that a robust model is chosen based on its relevance for human risk assessment. Since most of the genotoxicity and carcinogenicity testing data originates from rodents, it is important that there is a certain degree of conservation of the toxic response following chemical exposure among rodents and humans. In Chapter 2 the robust conserved genotoxic response among three frequently used in vitro models was investigated, following exposure to a prototypical GTX compound, explicitly cisplatin ${ }^{39}$. Transcriptomic data of cisplatin-exposed primary mouse hepatocytes (PMH), mouse embryonic stem cells (mESC) and human hepatocellular carcinoma cell line (HepG2) for 24h and $48 \mathrm{~h}$ were compared. Across these models, 44 conserved differentially expressed genes (DEGs) could be identified, which were shown to be involved in characteristic GTX-related processes (e.g. apoptosis, cell cycle, DNA damage response and DNA repair). The importance of these conserved genes was supported when they were benchmarked against transcriptomics data coming from GTX-exposed primary human hepatocytes (PHH). The regulation of the observed DEGs was thus indicated to be conserved among the three in vitro models originating from mouse and human. The 4 transcription factors (TFs) HNF4- $\alpha$, SP-1, c-MYC and p53, appeared capable of regulating $\pm 50 \%$ of all DEGs across all models and exposure periods. From Chapter 2 we therefore concluded that the robust genotoxic response was mainly determined by a conserved complex regulatory network of 4 TFs. In addition, due to a low transcriptomic response in the non-liver model mESC, and the reduced metabolic competence of HepG2 cells, the PMH model was chosen as an appropriate model for our further exposure studies using GTX and NGTX compounds.

\section{Understanding of regulatory networks underlying mechanisms of toxicity induced by GTXC and NGTXC}

The basic principle of developing an improved in vitro model for genotoxicity and carcinogenicity testing is that it should be able to differentiate GTXC from NGTXC. In order to accomplish this, the regulatory mechanisms underlying the toxic and carcinogenic effects of GTXC and NGTXC, in our case leading to HCC, need to be further explored. For these purposes, the robust in vitro liver model PMH, identified in Chapter 2, was advanced within Chapter 3. In this study, PMH were exposed for $24 \mathrm{~h}$ and $48 \mathrm{~h}$ to three prototypical GTXC (i.e. aflatoxin B1, benzo[a]pyrene and cisplatin) and three NGTXC $(2,3,7,8$ tetrachloordibenzodioxine, cyclosporine $\mathrm{A}$ and $\mathrm{W}_{\mathrm{y}-14,643)}$ with different modes of action $(\mathrm{MOA})^{40}$. Based on transcriptomics data from these exposed $\mathrm{PMH}$, discriminative gene sets, microRNAs and processes could be obtained. After 48h of exposure, these observations were most apparent. One microRNA-target gene interaction, particularly mmu-miR-503-5p with Cyclin D2, was shown to be distinctly expressed between PMH treated with GTXC or 
NGTXC. This interaction seemed to function within the p53 signaling pathway and may play a role in metabolism-related processes. Especially for PMH treated with NGTXC this interaction may be relevant, as it is expected to have an oncogenic function by stimulating Ccnd2, possibly leading to a tumorigenic cell cycle progression. For PMH treated with GTXC, mainly compound-specific microRNA-target gene interactions could be identified. The interaction mmu-miR-301b-3p with Papss 2 seems to be relevant in PMH treated with aflatoxin B1, while mmu-miR-29b-3p with Col4a2 and mmu-miR-24-3p with Flna were more prominent following benzo(a)pyrene exposure. These interactions mainly participate in cell cycle arrest and the impairment of the DNA damage repair, which are important hallmarks of GTXinduced carcinogenesis. From the results obtained in Chapter 3 we concluded that microRNAs, in addition to the TFs obtained from Chapter 2, represent yet another relevant intracellular regulatory level in chemical carcinogenesis induced by GTXC and NGTXC.

\section{Usefulness of microRNA expression profiles of exposed PMH for genotoxicity or carcinogenicity testing}

For the development of an accurate and sensitive alternative hepatic in vitro system for genotoxicity and carcinogenicity testing it is important that it is able to discriminate between compounds with different characteristics. Current in vitro tests generate a high degree of false positives; indicating that a compound is genotoxic in vitro but not in the in vivo situation. Furthermore, they are not able to detect non-genotoxic carcinogens (NGTXC). Consequently, there is a high demand for developing assays which are more reliable in predicting the genotoxic and carcinogenic potential of a compound in humans. In Chapter 4 we first aimed at developing an alternative in vitro model which is able to accurately separate PMH treated with GTX compounds from NGTX compounds. A second prediction model was generated to reliably discriminate PMH treated with NGTXC from non-carcinogens (NC). Since, following up on results described in Chapter 3, microRNAs seem to play an important role in regulatory networks in chemical carcinogenesis induced by GTX and NGTX compounds, we hypothesized that the response-patterns of the affected microRNAs could be used for prediction purposes. We therefore extended the list of compounds used within Chapter $\mathbf{3}$ with 15 additional compounds of either the GTX or NGTX toxicity class, representing prototypical model compounds. In order to compare the usefulness of microRNA expression patterns for classification purposes, the results of the microRNA-based approach were compared with mRNA-based prediction analysis outcomes. Ten different sets of training and test compounds were generated in order to avoid the selection of candidate mRNAs or microRNAs to be dependent on the composition of the training and test set. In addition, a leave-one-out based pre-selection was executed in order to prevent the identification of candidate mRNAs or microRNAs to depend upon compound-specific effects. Differentially expressed mRNAs (DEGs) and differentially expressed microRNAs (DE-miRs) overlapping between the different sets of training and test compounds, were then identified. With only these specific candidates, prediction analysis was performed, using the ten different training and test set compositions, by applying the nearest shrunken centroid method within the Prediction Analysis for Microarrays (PAM) program. Unfortunately, we were unable to identify any overlapping DEmiRs which were able to accurately predict genotoxicity or (non-genotoxic) carcinogenicity in vivo. By contrast, in particular after $48 \mathrm{~h}$ of exposure, 2 candidate DEGs were identified which were able to predict in vivo genotoxicity. An average accuracy of $93 \%$ (a sensitivity of $75 \%$ and a specificity of $100 \%$ ) could be achieved for the overall prediction of compounds within the test set. Tributylinoxide (TBTO), which has an inconclusive GTX nature, was misclassified. Also for predicting in vivo non-genotoxic carcinogenicity, a prediction model was based on mRNA expression profiles. 3 DEGs could be identified after $48 \mathrm{~h}$ of exposure which resulted 
in a model which was able to predict NGTX carcinogenicity with an accuracy of $88 \%$ (a sensitivity of $83 \%$ and a specificity of $93 \%$ ). Despite the relatively high accuracy and sensitivity of the prediction model, Wy-14,643 (Wy) was misclassified. The carcinogenic potential of Wy in $\mathrm{PMH}$ is however questionable in the absence of neighboring Kupffer cells and could therefore be missed within the currently applied in vitro liver model. From the findings within Chapter 4 we concluded that mRNA-based prediction of genotoxicity and non-genotoxic carcinogenicity outperforms equivalent microRNA-based prediction. With the currently used set of compounds, microRNA-based prediction of these traits is not feasible, however; a wider range of prototypical compounds would need to be investigated to further validate these negative findings.

\section{Regulatory interaction networks related with GTX exposure and associated with HCC}

Another requirement for the development of an alternative in vitro system is that it is relevant for the in vivo human situation. The best option for such a robust model is the use of primary human hepatocytes $(\mathrm{PHH})^{41,42}$. Inter-individual differences however often exist between different hepatocyte donors and therefore it is extremely complicated to draw any conclusions from these results which could be relevant for human risk assessment in general. A solution for this problem is culturing hepatocytes from different donors together in one petri dish in order to obtain a more general GTX response which might be more useful for chemical risk assessment of carcinogens in humans. Aflatoxin B1 (AFB1), produced by Aspergillus fungi, is one of the most potent GTX hepatocarcinogens, which is able to induce AFB1-adducts with for example DNA, initiating mutations ultimately leading to HCC development. Less is however known about the potential epigenetic changes that GTXC, specifically AFB1, might induce affecting regulatory networks, also involving microRNAs, which could also lead to the induction of HCC. Alterations of epigenetic DNA methylation patterns (attachment or the removal of specific methyl groups to the DNA affecting gene expression) might in combination with microRNA expression changes be characteristic for a particular type of GTX exposure. Specific affected regulatory genes or networks may therefore be used as potential candidates for genotoxicity and carcinogenicity testing. In Chapter 5, a pool of cryopreserved PHH was treated with two doses (low: $0.3 \mu \mathrm{M}$ and moderate: $1 \mu \mathrm{M}$ ) of the prototypical GTXC, aflatoxin B1 (AFB1). Following a repetitive 5-day exposure period the $\mathrm{PHH}$ were harvested and microRNA and mRNA expression changes were measured together with DNA methylation changes within the promotor region of the respective genes. In order to assess the relevance of the observed in vitro transcriptomic changes with respect to the risk of developing HCC in vivo, results were benchmarked against a specific gene expression signature profile retrieved from HCC patients ${ }^{43}$. In addition, results were compared with a list of known AFB1-responsive genes obtained from the Comparative Toxicogenomics Database $(\mathrm{CTD})^{44}$. One remarkable finding was that $\mathrm{PHH}$ exposed for 5 days to $0.3 \mu \mathrm{M}$ of AFB1 showed more changes on the DNA methylation level in comparison with cells exposed to 1 $\mu \mathrm{M}$ of AFB1. Also more DNA hypomethylation changes occurred due to AFB1 treatment in general than DNA hypermethylation alterations. When comparing $\mathrm{PHH}$ exposed to $0.3 \mu \mathrm{M}$ and $1 \mu \mathrm{M}$ of AFB1, a large set of genes could be identified which were commonly affected on the DNA methylation level. Once the methylation data were combined with the transcriptomics data, a much smaller set of affected genes remained indicating that changes on the DNA methylation level are not always directly affecting gene expression levels. When AFB1-induced DEGs, which were affected on the DNA methylation level, were combined with DE-miRs, HCC-related biological processes such as metabolism, nucleic acid metabolism, DNA repair, cell cycle, apoptosis, immune response and ion transport were retrieved. Specific pathways within these AFB1-induced biological processes appeared involved in signal 
transduction cascades. Here, in particular, a number of microRNA-targeted genes could be identified comprising ARHGAP35, ILK, PPP2R1A, RB1, TFAP2A, HIST1H4D, RHOA, CDK4 and UBC. Deregulation of these genes and signal transduction cascades represent important hallmarks of $\mathrm{HCC}^{45}$. The findings from Chapter $\mathbf{5}$ contribute to a better understanding of GTX-induced regulatory network changes which potentially could lead to the development of HCC.

\section{Persistence of GTX-induced alterations in regulatory networks relevant for onset of HCC}

In order to conclude whether in vitro carcinogen-induced changes within regulatory networks, are relevant for the in vivo disease state and are therefore useful for human risk assessment, it is essential that these changes are persistent and remain irreversible when the carcinogen exposure is removed. In Chapter $\mathbf{6}$ we therefore investigated whether the changes of the transcriptome and the microRNAome observed in a pool of cryopreserved PHH after a 5-day challenge with a $1 \mu \mathrm{M}$ of AFB1, persist after carcinogen treatment is withdrawn (=washout period). Also in Chapter 6 as well as Chapter 7 transcriptomic results were benchmarked against an HCC signature profile obtained from liver transcriptomic data from HCC patients, and a list of known AFB1-inducible genes. Especially a regulatory gene network directed by two persistently HCC-related microRNAs, hsa-miR-34b-5 $\mathrm{p}^{46,47}$ and hsa-miR-222-3p $\mathrm{p}^{25}$, seemed to be involved in some characteristic AFB1-induced processes referring to cancer-associated metabolism changes and regulation of gene expression. Additionally, hsa-miR-34a-5p, hsamiR-96-5p and hsa-miR-30a-3p which are only differentially expressed after the termination of the $1 \mu \mathrm{M}$ AFB1-treatment, seem to regulate HCC-related genes which are persistently expressed. In parallel, persistent changes in PHH induced by a $0.3 \mu \mathrm{M}$ AFB1 challenge on the methylome, microRNAome, and transcriptome were further investigated in Chapter 7 when the carcinogen exposure was ended. At this lower AFB1 dose, no DE-miRs could be identified which were still affected after the 3-day washout period. By contrast, upon combining transcriptomics data with results on DNA methylation, a variety of persistently affected hyperand hypomethylated genes was identified which also seemed changed on the transcriptome level. Six persistently hypomethylated and upregulated genes (i.e. TXNRD1, PCNA, CCNK, DIAPH3, RAB27A and HIST1H2BF) were identified which may be involved in several carcinogenic events. The findings from Chapter $\mathbf{6}$ and Chapter $\mathbf{7}$ may therefore contribute to a better understanding of persistent GTX-induced microRNA-directed changes within epigenetic regulatory networks, which could be predictive for the ultimate onset of HCC.

\section{Conclusion}

In general, our aim to elucidate the participation of microRNA and epigenetic changes in transcriptomic responses induced by GTX and NGTX compounds in in vitro liver models has been achieved. We were able to identify robust responses, between several in vitro models, following exposure to the GTX compound cisplatin (Chapter 2). These were mainly determined by a conserved complex regulatory network of 4 TFs, thereby highlighting the importance of regulatory mechanisms in the GTX response. Changes in TF regulatory networks were also studied before, as they are thought to be conserved during evolution and have been shown to play an important role in the transcriptomic response of HepG2 cells to exposure to the GTX compound benzo[a]pyrene ${ }^{48,49}$. This knowledge on the role of regulatory networks in chemical carcinogenesis from Chapter 2 was extended with two additional studies in which more chemical compounds were investigated in the robust in vitro liver model, PMH. MicroRNA and mRNA expression changes were taken into account. In the first study (Chapter 3), in which PMH were investigated following exposure to 3 GTXC and 3 NGTXC, 
especially mmu-microRNA-503-5p was found to behave differently between the two groups of carcinogens. Though, mainly compound-specific microRNA changes were observed. From the second, more elaborate, study (Chapter 4) we also concluded that since most of the microRNA expression changes, occurring after short-term exposure, are compound-specific their use within prediction analysis for in vivo genotoxicity or carcinogenicity testing is not successful. These findings were confirmed with in vivo results obtained by Melis and colleagues 50 who were unable to accurately discriminate GTXC from NGTXC using microRNA expression profiles from 7-day exposed mice. MicroRNA expression changes are, however, valuable when studying MOA of especially NGTXC as was observed in Chapter 3. From this study we also concluded that the discrimination of PMH treated with GTX compounds from cells treated with NGTX compounds or NGTXC-treated PMH from NC-exposed cells is most successful using gene expression changes. Discriminating DE-mRNAs were identified which appeared relevant for GTX and carcinogenic exposures. Therefore, despite that microRNA expression changes are not useful as predictive candidates for in vivo genotoxicity and carcinogenicity testing, the analysis of carcinogen-induced changes on the microRNA level, in combination with their affected target genes, has actually provided novel insights into the biological mechanisms of for example NGTX carcinogenesis, affecting tumorigenic cell cycle progression via mmu-miR-503-5p and cyclin D2, ultimately leading to HCC development. This is in concordance with the hypothesis that chemically induced microRNA expression changes may be representative for the alterations occurring in $\mathrm{HCC}^{22}$. MicroRNA expression changes in combination with modifications of expression of their target genes, represented as microRNA-directed regulatory networks, may therefore be useful in the development of adverse outcome pathways (AOPs) within human risk assessment. From the studies performed with $\mathrm{PHH}$ (Chapter 5-7), we conclude that specific changes on the microRNA expression level, in addition to transcriptomic changes, may be persistent when the GTXC treatment is terminated (Chapter 6). This is especially present when PHH were treated with $1 \mu \mathrm{M}$ with the model GTXC, AFB1. Next to microRNAs, also DNA methylation changes seem to be persistently changed, following $0.3 \mu \mathrm{M}$ of AFB1 exposure, thereby affecting the expression of several genes which contribute to a disease signature which is characteristic for HCC (Chapter 7). Benchmarking of gene expression results with a specific pre-existing signature profile retrieved from HCC patients is fairly crucial in this step. The finding that, HCC-related persist changes occur in vitro in PHH on the transcriptome, microRNA and/or methylome level following termination of carcinogen treatment, is relatively new within the field of toxicogenomics. Especially these persistently epigenetically affected genes and microRNAs may be the most relevant potential biomarkers for chemically induced (hepato)carcinogenesis. Therefore particularly this mechanistic information on these HCC-related persistently affected epigenetic- and microRNA-directed regulatory networks may be used for the development of AOPs contributing to the future risk assessment of human carcinogens.

\section{Limitations and future recommendations}

Promising results have been obtained that might provide more insight into the epigenetic- and microRNA-directed regulatory mechanisms that participate in (hepato-)carcinogenesis induced by GTXC and NGTXC. This knowledge might, on the long run, be used for the development of AOPs important within human risk assessment of chemicals, nutrients and pharmaceuticals. These mechanistic results, on the role microRNAs and epigenetics in chemical carcinogenesis, are still preliminary and may therefore form the basis of future studies. Future studies should focus on validating the obtained results for prediction purposes on the microRNA expression level, within preferably PHH, by evaluating more compounds. This should verify if the microRNA expression patterns are indeed not valuable for prediction purposes. In addition, 
another method could be applied which focusses on network-based pre-selecting or ranking of relevant microRNAs for genotoxicity and non-genotoxic carcinogenicity. Recently, such a method has been developed for the ranking of microRNAs based on their relevance in a particular disease ${ }^{51}$. Preferably GTXC and NGTXC and NC should be used and their different MOA should be taken into account for example for read-across initiatives such as connectivity mapping ${ }^{52,53}$. Furthermore, persistency of the obtained effects should be considered since these are particularly relevant for the development of $\mathrm{HCC}^{21,54}$. Therefore, well-designed time series studies should be performed which focus on the series of events that take place following carcinogen treatment. In this way, more information can be obtained on the response-time of microRNAs, in PHH, after exposure to different GTXC and NGTXC. Additionally, the effect of the microRNA-target gene interactions should be validated on the protein level and in $\mathrm{PHH}$ specifically to see the actual effect of the microRNA suppression on gene expression ${ }^{55}$. In addition, the results obtained within $\mathrm{PHH}$ should be extended with more compounds of different classes to obtain more information on the epigenetic- and microRNA-directed regulatory networks affected following exposure to GTXC or NGTXC. To cover the epigenetic mechanisms completely also histone acetylation changes should be measured. Specific epigenetic- and microRNA-directed gene expression foot-prints might be obtained which may then form the basis for the development of more mechanistically-based AOPs. Therefore, the obtained results should be linked with toxicological endpoints (i.e. phenotypic anchoring $)^{56}$, which are characteristic for a particular class of compounds e.g. DNA-adduct formation or oxygen radical formation. Moreover, additional donors should be used to further support the idea that the results obtained are representative for the human situation. As alternative systems, also 3 dimensional organotypical liver organoid and spheroid ${ }^{57}$ cell culture systems such as pluripotent stem cell-derived hepatocytes ${ }^{58}$ or hepatocyte co-cultures might be used, which are more representative for the in vivo human liver ${ }^{59}$. These 3 dimensional liver organoid/spheroid cell culture systems are to be preferred considering their restored extracellular matrix and thereby hepatocyte cell polarity. By re-establishing these characteristics of the liver, cells seem to maintain their metabolic competence and may be cultured for a longer period of time ${ }^{59}$. Mainly the co-culturing of human hepatocytes with for example Kupffer cells (resident macrophages), sinusoidal endothelial cells, and stellate cells (stromal cells) seems successful in mimicking the in vivo human situation ${ }^{59}$. By applying such a co-culture model of hepatocytes, which can be kept in culture for a longer period of time, may help further studying the observed persistent effects of carcinogen exposure.

The current findings of this thesis in combination with additional cross-omics based future experiments might provide new insight into the regulatory networks contributing to the MOA of chemical carcinogens which could be of predictive value for human risk assessment. 


\section{References}

1. Ferlay, J. et al. Cancer incidence and mortality worldwide: sources, methods and major patterns in GLOBOCAN 2012. Int J Cancer 136, E359-86 (2015).

2. Kew, M.C. Aflatoxins as a cause of hepatocellular carcinoma. J Gastrointestin Liver Dis 22, 305-10 (2013).

3. Ashby, J. Use of short-term tests in determining the genotoxicity or nongenotoxicity of chemicals. IARC Sci Publ, 135-64 (1992).

4. Silva Lima, B. \& Van der Laan, J.W. Mechanisms of nongenotoxic carcinogenesis and assessment of the human hazard. Regul Toxicol Pharmacol 32, 135-43 (2000).

5. Ames, B.N., Lee, F.D. \& Durston, W.E. An improved bacterial test system for the detection and classification of mutagens and carcinogens. Proc Natl Acad Sci US A 70, 782-6 (1973).

6. Kirkland, D., Reeve, L., Gatehouse, D. \& Vanparys, P. A core in vitro genotoxicity battery comprising the Ames test plus the in vitro micronucleus test is sufficient to detect rodent carcinogens and in vivo genotoxins. Mutat Res 721, 27-73 (2011).

7. Matsushima, T. et al. Validation study of the in vitro micronucleus test in a Chinese hamster lung cell line (CHL/IU). Mutagenesis 14, 569-80 (1999).

8. Moller, P. Genotoxicity of environmental agents assessed by the alkaline comet assay. Basic Clin Pharmacol Toxicol 96 Suppl 1, 1-42 (2005).

9. Shelby, M.D. \& Witt, K.L. Comparison of results from mouse bone marrow chromosome aberration and micronucleus tests. Environ Mol Mutagen 25, 302-13 (1995).

10. Kirkland, D. et al. How to reduce false positive results when undertaking in vitro genotoxicity testing and thus avoid unnecessary follow-up animal tests: Report of an ECVAM Workshop. Mutat Res 628, 31-55 (2007).

11. Hernandez, L.G., van Steeg, H., Luijten, M. \& van Benthem, J. Mechanisms of non-genotoxic carcinogens and importance of a weight of evidence approach. Mutat Res 682, 94-109 (2009).

12. Ennever, F.K. \& Lave, L.B. Implications of the lack of accuracy of the lifetime rodent bioassay for predicting human carcinogenicity. Regul Toxicol Pharmacol 38, 52-7 (2003).

13. Kirkland, D. Improvements in the reliability of in vitro genotoxicity testing. Expert Opin Drug Metab Toxicol 7, 1513-20 (2011).

14. Kitano, H. Computational systems biology. Nature 420, 206-10 (2002).

15. Kleinjans, J. Toxicogenomics-based cellular models : alternatives to animal testing for safety assessment, xviii, $348 \mathrm{p}$. (Elsevier/AP, Amsterdam, 2014).

16. Piersma, A.H. et al. A critical appraisal of the process of regulatory implementation of novel in vivo and in vitro methods for chemical hazard and risk assessment. Crit Rev Toxicol 44, 876-94 (2014).

17. Ankley, G.T. et al. Adverse outcome pathways: a conceptual framework to support ecotoxicology research and risk assessment. Environ Toxicol Chem 29, 730-41 (2010).

18. National Research Council (U.S.). Committee on Applications of Toxicogenomic Technologies to Predictive Toxicology. Applications of toxicogenomic technologies to predictive toxicology and risk assessment, xxii, 275 p. (National Academies Press, Washington, D.C., 2007).

19. Olden, K. Toxicogenomics--a new systems toxicology approach to understanding of gene-environment interactions. Ann N Y Acad Sci 1076, 703-6 (2006).

20. Goodman, J.I. et al. What do we need to know prior to thinking about incorporating an epigenetic evaluation into safety assessments? Toxicol Sci 116, 375-81 (2010).

21. Mirbahai, L. \& Chipman, J.K. Epigenetic memory of environmental organisms: a reflection of lifetime stressor exposures. Mutat Res Genet Toxicol Environ Mutagen 764-765, 10-7 (2014).

22. Izzotti, A. \& Pulliero, A. The effects of environmental chemical carcinogens on the microRNA machinery. International Journal of Hygiene and Environmental Health 217, 601-627 (2014).

23. Giordano, S. \& Columbano, A. MicroRNAs: new tools for diagnosis, prognosis, and therapy in hepatocellular carcinoma? Hepatology 57, 840-7 (2013).

24. Gramantieri, L. et al. MicroRNA involvement in hepatocellular carcinoma. J Cell Mol Med 12, 2189-204 (2008).

25. Karakatsanis, A. et al. Expression of microRNAs, miR-21, miR-31, miR-122, miR-145, miR-146a, miR200c, miR-221, miR-222, and miR-223 in patients with hepatocellular carcinoma or intrahepatic cholangiocarcinoma and its prognostic significance. Mol Carcinog 52, 297-303 (2013).

26. Li, J., Wang, Y., Yu, W., Chen, J. \& Luo, J. Expression of serum miR-221 in human hepatocellular carcinoma and its prognostic significance. Biochem Biophys Res Commun 406, 70-3 (2011).

27. Pineau, P. et al. miR-221 overexpression contributes to liver tumorigenesis. Proc Natl Acad Sci U S A 107, 264-9 (2010).

28. Sun, J., Lu, H., Wang, X. \& Jin, H. MicroRNAs in hepatocellular carcinoma: regulation, function, and clinical implications. ScientificW orldJournal 2013, 924206 (2013). 
29.

30.

31.

32.

33.

34.

35.

36.

37.

38.

39.

40.

41.

42.

43.

44.

45.

46.

47.

48.

49.

50.

51.

52.

53.

54.

55.

56.

57.

58.

59.

Xiong, Y. et al. Effects of microRNA-29 on apoptosis, tumorigenicity, and prognosis of hepatocellular carcinoma. Hepatology 51, 836-45 (2010).

Baylin, S.B., Herman, J.G., Graff, J.R., Vertino, P.M. \& Issa, J.P. Alterations in DNA methylation: a fundamental aspect of neoplasia. Adv Cancer Res 72, 141-96 (1998).

Bird, A. DNA methylation patterns and epigenetic memory. Genes Dev 16, 6-21 (2002).

Bollati, V. et al. Exposure to metal-rich particulate matter modifies the expression of candidate microRNAs in peripheral blood leukocytes. Environ Health Perspect 118, 763-8 (2010).

Cheng, Y. et al. Correlation of $\mathrm{CpG}$ island methylator phenotype with poor prognosis in hepatocellular carcinoma. Exp Mol Pathol 88, 112-7 (2010).

Hernandez-Vargas, H. et al. Hepatocellular Carcinoma Displays Distinct DNA Methylation Signatures with Potential as Clinical Predictors. PLoS ONE 5, e9749 (2010).

Lee, Y.W. et al. Carcinogenic nickel silences gene expression by chromatin condensation and DNA methylation: a new model for epigenetic carcinogens. Mol Cell Biol 15, 2547-57 (1995).

Shen, J. et al. Genome-wide aberrant DNA methylation of microRNA host genes in hepatocellular carcinoma. Epigenetics 7, 1230-7 (2012).

Zhang, Y.J. et al. Global hypomethylation in hepatocellular carcinoma and its relationship to aflatoxin B(1) exposure. World J Hepatol 4, 169-75 (2012).

Osella, M., Riba, A., Testori, A., Cora, D. \& Caselle, M. Interplay of microRNA and epigenetic regulation in the human regulatory network. Front Genet 5, 345 (2014).

Rieswijk, L., Lizarraga, D., Brauers, K.J., Kleinjans, J.C. \& van Delft, J.H. Characterisation of cisplatininduced transcriptomics responses in primary mouse hepatocytes, HepG2 cells and mouse embryonic stem cells shows conservation of regulating transcription factor networks. Mutagenesis 29, 17-26 (2014).

Rieswijk, L. et al. Evaluating microRNA profiles reveals discriminative responses following genotoxic or non-genotoxic carcinogen exposure in primary mouse hepatocytes. Mutagenesis (2015).

Lecluyse, E.L. \& Alexandre, E. Isolation and culture of primary hepatocytes from resected human liver tissue. Methods Mol Biol 640, 57-82 (2010).

Hewitt, N.J. et al. Primary hepatocytes: current understanding of the regulation of metabolic enzymes and transporter proteins, and pharmaceutical practice for the use of hepatocytes in metabolism, enzyme induction, transporter, clearance, and hepatotoxicity studies. Drug Metab Rev 39, 159-234 (2007).

Caiment, F., Tsamou, M., Jennen, D. \& Kleinjans, J. Assessing compound carcinogenicity in vitro using connectivity mapping. Carcinogenesis 35, 201-7 (2014).

Davis, A.P. et al. The Comparative Toxicogenomics Database: update 2013. Nucleic Acids Res 41, D1104-14 (2013).

Hanahan, D. \& Weinberg, Robert A. Hallmarks of Cancer: The Next Generation. Cell 144, 646-674 (2011). Hermeking, H. The miR-34 family in cancer and apoptosis. Cell Death Differ 17, 193-9 (2010).

$\mathrm{Xu}, \mathrm{Y}$. et al. A potentially functional polymorphism in the promoter region of miR-34b/c is associated with an increased risk for primary hepatocellular carcinoma. Int J Cancer 128, $412-7$ (2011).

Vaquerizas, J.M., Kummerfeld, S.K., Teichmann, S.A. \& Luscombe, N.M. A census of human transcription factors: function, expression and evolution. Nat Rev Genet 10, 252-63 (2009).

van Delft, J.H. et al. Time series analysis of benzo[A]pyrene-induced transcriptome changes suggests that a network of transcription factors regulates the effects on functional gene sets. Toxicol Sci 117, 381-92 (2010). Melis, J.P. et al. In vivo murine hepatic microRNA and mRNA expression signatures predicting the (non)genotoxic carcinogenic potential of chemicals. Arch Toxicol (2014).

Le, D.H. Network-based ranking methods for prediction of novel disease associated microRNAs. Comput Biol Chem 58, 139-148 (2015).

Caiment, F., Tsamou, M., Jennen, D. \& Kleinjans, J. Assessing compound carcinogenicity in vitro using connectivity mapping. Carcinogenesis Advance Access published August 12, 2013, doi:10.1093/carcin/bgt278 (2013).

Lamb, J. et al. The Connectivity Map: using gene-expression signatures to connect small molecules, genes, and disease. Science 313, 1929-35 (2006).

Heijmans, B.T. et al. Persistent epigenetic differences associated with prenatal exposure to famine in humans. Proc Natl Acad Sci US A 105, 17046-9 (2008).

Selbach, M. et al. Widespread changes in protein synthesis induced by microRNAs. Nature 455, 58-63 (2008).

Waters, M.D. \& Fostel, J.M. Toxicogenomics and systems toxicology: aims and prospects. Nat Rev Genet 5, 936-948 (2004).

Hynds, R.E. \& Giangreco, A. Concise review: the relevance of human stem cell-derived organoid models for epithelial translational medicine. Stem Cells 31, 417-22 (2013).

Davidson, M.D., Ware, B.R. \& Khetani, S.R. Stem cell-derived liver cells for drug testing and disease modeling. Discov Med 19, 349-58 (2015).

Soldatow, V.Y., LeCluyse, E.L., Griffith, L.G. \& Rusyn, I. In vitro models for liver toxicity testing. Toxicology research 2, 23-39 (2013). 
C

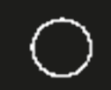

0

0

○

- Addendum I

00000

0 Samenvatting en Algemene discussie

O

O

0

C

O

○

$\bigcirc$

00

○

()

○

(

0

()

0

)

C

○

$\mathrm{O}$

00

00

()

000

0

0

5 


\section{Samenvatting en Algemene discussie}

Wereldwijd is kanker de meest voorkomende, ziekte-gerelateerde, doodsoorzaak. Er wordt verwacht dat het aantal mensen dat door deze ziekte overlijdt in de komende twintig jaar flink zal stijgen ${ }^{1}$. Leverkanker, met name hepatocellulair carcinoma (HCC), staat in de wereld op de zesde plaats van meest gediagnostiseerde vormen van kanker en op nummer 2 als zijnde de dodelijkste vorm van kanker ${ }^{1}$. Vooral in de minder ontwikkelde landen vormt HCC een enorm gezondheidsprobleem. HCC wordt, behalve door genetische elementen, veroorzaakt door met name biologische (bv. hepatitis-B en -C virussen) en omgevingsfactoren (bv. aflatoxine B1 blootstelling) ${ }^{2}$. Ongeveer een derde van het aantal nieuwe gevallen zou voorkomen kunnen worden door een zorgvuldige controle en het vermijden van blootstelling met carcinogenen uit de omgeving (bv. chemicaliën, geneesmiddelen en voedingsstoffen) ${ }^{1}$.

Traditioneel worden omgevingscarcinogenen gescheiden in twee verschillende groepen, gebaseerd op het verschil in werkingsmechanismse van de respectievelijke stoffen, namelijk in: genotoxische (GTX) en niet-genotoxische (NGTX) carcinogenen ${ }^{3}$. GTX-carcinogenen (GTXC) kunnen een directe reactie aangaan met het DNA, waarbij ze mutaties kunnen initiëren die kunnen leiden tot de activatie van kanker-gerelateerde processen. NGTXcarcinogenen (NGTXC) bevorderen kanker via diverse niet-DNA-reactieve mechanismen, bv. door de inductie van oxidatieve stress of via binding aan de arylhydrocarbon receptor (AhR), dan wel aan de peroxisoom proliferator geactiveerde receptor alfa (PPAR- $\alpha)^{4}$. Om een goede controle te garanderen en de blootstelling aan omgevingscarcinogenen te voorkomen, is nauwkeurige en gevoelige chemische risicobeoordeling van genotoxiciteit en carcinogeniteit noodzakelijk. Dit om te voorkomen dat gevaarlijke stoffen op de markt worden gebracht, om de humane blootstelling aan carcinogenen te reduceren en om het aantal nieuwe gevallen van bv. HCC te verminderen.

Nieuwe chemicaliën, geneesmiddelen of voedingsstoffen worden gewoonlijk getest in een reeks van in vitro genotoxiciteitstesten (bv. de bacteriële mutageniteitstest van Ames, muis lymphoma mutageen assay (MLA), micronucleus (MN) test en de chromosomale aberraties (CA) test), welke representatief zouden moeten zijn voor het carcinogene effect op de langere termijn ${ }^{5-9}$. Zodra een chemische stof, geneesmiddel of voedingsstof positief is bevonden op basis van deze in vitro testen, zullen er additionele in vivo testen moeten worden uitgevoerd. De huidige in vitro genotoxiciteitstesten worden echter gekenmerkt door een groot aantal vals positieven ${ }^{10}$. Een vals positief resultaat in vitro wil zeggen dat een stof positief wordt bevonden in de in vitro situatie, maar daadwerkelijk negatief is in vivo. Misschien nog wel belangrijker, NGTXC-verbindingen worden niet gedetecteerd ${ }^{11}$. In vivo validatie van positieve resultaten van de in vitro genotoxiciteitstesten wordt standaard uitgevoerd door middel van een twee jaar durende studie bij knaagdieren (bv. muizen of ratten). Deze tests zijn langdurig, kosten bovendien veel geld en eisen veelal onnodig het leven van een groot aantal proefdieren. Een ander nadeel van deze tweejarige studies bij knaagdieren is dat ze vrij beperkt zijn in hun voorspellend vermogen $(\sim 50 \%) 12$ van in vivo carcinogeniteit bij mensen. In vivo testen genereren een hoge mate van vals positieven (specifieke knaagdier carcinogenen) en vals negatieven (exclusief humane carcinogenen) ${ }^{12}$. Zowel in vitro als in vivo genotoxiciteits- en carcinogeniteitstesten gaan dus gepaard met een hoog aantal vals positieven. Wegens het gebrek aan nauwkeurige en gevoelige testen, die in staat zijn om de genotoxische en (nietgenotoxisch) carcinogene potentiaal van chemische stoffen in humane risicobeoordeling te evalueren, is er een grote vraag naar betrouwbare alternatieve systemen en assays ${ }^{13}$.

De focus van de humane risicobeoordeling is daarom verschoven naar een benadering die meer is gericht is op de systeembiologie ${ }^{14-16}$. Het basis principe achter deze nieuwe strategie is 
dat door het verkrijgen van een beter begrip van de biologische mechanismen die ten grondslag liggen aan genotoxiciteit en carcinogeniteit, in een meer nauwkeurige en gevoelige voorspelling kan worden voorzien, welke bruikbaar is voor de in vivo humane risicobeoordeling ${ }^{17}$. Het vakgebied van toxicogenomics, waarbij veelal op -omics gebaseerde high-throughput technologieën worden toegepast, lijkt veelbelovend voor het ontwikkelen van dergelijke alternatieve systemen ${ }^{18}$. Vooral met behulp van de integratie van meerdere lagen van biologische systemen kan meer inzicht worden verkregen wat kan bijdragen tot het beter begrijpen van de onderliggende carcinogene mechanismen ${ }^{19}$. Processen die de transcriptie (d.w.z. DNA-methylatie) en de translatie (d.w.z. regulatie door korte niet-coderende microRNA's) reguleren, zouden hierbij in beschouwing moeten worden genomen 20-22. Bovendien zou de identificatie van belangrijke regulatoire genen en processen die een rol spelen in zogenoemde "Adverse Outcome Pathways (AOPs)" 17, relevant kunnen zijn voor voorspellingsdoeleinden binnen de humane risicobeoordeling ${ }^{20-22}$. Veel van deze regulatoire mechanismen zijn afwijkend in het geval van ziekte, bv. in HCC. Sommige microRNA's met een tumor-onderdrukkende functie worden omlaag gereguleerd binnen $\mathrm{HCC}^{23-29}$. In een groot aantal studies naar HCC is aangetoond dat de promotor van specifieke tumor-onderdrukkende genen vaak een DNA-hypermethylatie vertonen, alsmede een globale genoom DNAhypomethylatie ${ }^{30-37}$. De wisselwerking tussen de verschillende regulatoire lagen lijkt een grote invloed te hebben op het transcriptoom waarmee het lot van de cel wordt bepaald ${ }^{38}$. Chemische carcinogeen blootstelling lijkt ook deze regulatoire netwerken te beïnvloeden en kunnen zelfs, indien de effecten persisteren, voorspellend zijn voor de vroege tekenen van de ziekte, zoals $\mathrm{HCC}^{21}$. Het doel van dit proefschrift was dan ook om de rol van microRNA's en epigenetische veranderingen op het transcriptoom in deze regulatoire netwerken verder te ontrafelen na blootstelling aan GTX- en NGTX-verbindingen in de in vitro lever.

\section{Identificatie van een robuust in vitro lever model voor genotoxiciteit en carcinogeniciteitsbepalingen}

Bij de ontwikkeling van een nauwkeuriger in vitro systeem voor het testen van genotoxiciteit en carcinogeniteit, is het erg belangrijk dat een robuust model wordt gekozen, gebaseerd op zijn relevantie voor humane risicobeoordeling. Aangezien een groot aantal van de genotoxiciteitsen carcinogeniciteitstestresultaten afkomstig zijn van knaagdieren, is het belangrijk dat er een zekere mate van conservering van de toxische respons na blootstelling aan chemische stoffen is tussen knaagdieren en mensen. In hoofdstuk $\mathbf{2}$ is de robuuste geconserveerde genotoxische respons, tussen drie veelgebruikte in vitro modellen, na blootstelling aan een prototypische GTX-verbinding, cisplatine, verder onderzocht ${ }^{39}$. Transcriptoom resultaten, van cisplatine blootgestelde primaire muis hepatocyten $(\mathrm{PMH})$, muis embryonale stamcellen (mES) en humane hepatocellulaire carcinoma cellijn (HepG2) voor 24 uur en 48 uur, werden vergeleken. Vierenveertig geconserveerde differentieel tot expressie gekomen genen (DEGs) konden worden geïdentificeerd. Deze genen bleken te zijn betrokken bij karakteristieke GTXgerelateerde processen (bv. apoptose, celcyclus, DNA-schade respons en DNA-herstel). Het belang van deze geconserveerde genen werd verder ondersteund toen ze werden vergeleken met transcriptomics resultaten van GTX-blootgestelde primaire humane hepatocyten $(\mathrm{PHH})$. De regulatie van de vastgestelde DEGs zijn dus geconserveerd tussen de drie in vitro modellen afkomstig van muis en mens. De vier transcriptiefactoren (TFs) HNF4- $\alpha$, SP-1, c-MYC en p53, bleken in staat ongeveer vijftig procent van DEGs te reguleren voor alle modellen en blootstellingsperiodes. Uit hoofdstuk 2 kunnen we concluderen dat de robuuste genotoxische respons vooral werd bepaald door een complex geconserveerd regulatoir netwerk van 4 TFs. Daarnaast, als gevolg van een lage reactie op het transcriptoom niveau in het niet-lever model mES en de verminderde metabole capaciteit van HepG2 cellen, werd het PMH model gekozen 
als een geschikt model voor onze verdere blootstellingsonderzoeken met GTX- en NGTXverbindingen.

\section{Begrip van de regulerende netwerken die ten grondslag liggen aan het werkingmechanisme van GTXC- en NGTXC-verbindingen}

Het basisprincipe van het ontwikkelen van een verbeterd in vitro model voor genotoxiciteit en carcinogeniteitstesten is dat het GTXC moet kunnen onderscheiden van NGTXC. Om dit te bewerkstelligen zullen de regulatoire mechanismen die ten grondslag liggen aan de toxische en carcinogene effecten van GTXC en NGTXC, in ons geval leidend tot HCC, verder moeten worden onderzocht. Voor deze doeleinden is het robuuste in vitro lever model PMH, toegepast in hoofdstuk 2, opnieuw gebruikt in hoofdstuk 3. In deze studie werden PMH blootgesteld voor 24 en 48 uur aan drie prototypische GTXC- (d.w.z. aflatoxine B1, benzo [a] pyreen en cisplatine) en drie NGTXC (2,3,7,8-tetrachloordibenzodioxine, cyclosporine A en Wy-14643) verbindingen met verschillende werkingsmechanismen ${ }^{40}$. Op basis van transcriptoom resultaten van deze blootgestelde PMH konden onderscheidende sets van genen en microRNA's en kenmerkende processen worden verkregen. $\mathrm{Na} 48$ uur blootstelling, waren deze waarnemingen het meest duidelijk. Een microRNA-target gen interactie, in het bijzonder mmu-miR-503-5p met cycline D2, bleek duidelijk onderscheidend te zijn tussen PMH behandeld met GTXC- of NGTXC-verbindingen. Deze interactie lijkt belangrijk te zijn binnen de p53 signaleringsroute en zou een rol kunnen spelen in metabolisme-gerelateerde processen. Speciaal voor PMH behandeld met NGTXC-verbindingen is deze interactie relevant, omdat het naar verwachting een oncogene functie zal hebben door het stimuleren van Ccnd2. Dit kan leiden tot een tumorigene celcyclus progressie. Voor PMH behandeld met GTXCverbindingen, konden voornamelijk verbindingsspecifieke microRNA-target gen interacties worden geïdentificeerd. De interactie mmu-miR-301b-3p met Papss2 leek relevant te zijn in PMH behandeld met aflatoxine B1, terwijl mmu-miR-29b-3P met Col4a2 en mmu-miR-24-3p met FLNA meer prominent bleken te zijn na benzo(a)pyreen blootstelling. Deze interacties participeren voornamelijk in de celcyclus en dragen bij aan de remming van het DNAschadeherstel, welke belangrijke processen zijn in GTX-geïnduceerde carcinogenese. Uit de verkregen resultaten van hoofdstuk 3 concluderen wij dat microRNA's, naast de TFs van hoofdstuk 2 , weer een ander relevant intracellulair regulerend niveau vertegenwoordigen in de chemisch-geïnduceerde carcinogenese door GTXC- en NGTXC-verbindingen.

\section{De bruikbaarheid van microRNA expressie profielen van de blootgestelde PMH voor genotoxiciteits- of carcinogeniciteitsbepalingen}

Voor de ontwikkeling van een nauwkeuriger en gevoeliger alternatief, op lever-gebaseerd in vitro-systeem voor genotoxiciteits- en carcinogeniteitbepalingen, is het belangrijk dat het in staat is om onderscheid te maken tussen de verbindingen met verschillende eigenschappen. Huidige in vitro-testen genereren een groot aantal vals positieven; dit geeft aan dat de verbinding GTX is in vitro, maar niet in de in vivo situatie. Bovendien zijn de huidige testen niet in staat om NGTXC te detecteren. Ten gevolge daarvan, is er een grote vraag naar de ontwikkeling van alternatieve assays die meer betrouwbaar zijn in het voorspellen van de humane genotoxische en carcinogene potentiaal van verbindingen. In hoofdstuk 4 hebben we ons gericht op de ontwikkeling van een alternatief in vitro-model dat in staat is om nauwkeurig een onderscheid te maken tussen PMH behandeld met GTX-verbindingen van cellen behandeld met NGTXverbindingen. Een tweede voorspellend model werd gegenereerd om een betrouwbaar onderscheid te maken tussen PMH behandeld met NGTXC verbindingen en cellen blootgesteld aan niet-carcinogene stoffen (NC). Naar aanleiding van de resultaten beschreven in hoofdstuk 3, lijken microRNA's een belangrijke rol te spelen in regulatoire netwerken, 
essentieel in de chemische carcinogenese geïnduceerd door GTX- en NGTX-verbindingen. We veronderstelden om deze reden, dat de reactie-patronen van de veranderde microRNA's gebruikt kunnen worden voor voorspellingsdoeleinden. Daarom hebben we de lijst van stoffen die worden gebruikt in hoofdstuk 3 uitgebreid met vijftien additionele prototypisch modelverbindingen van ofwel de GTX of de NGTX toxiciteitsklassen. Om de bruikbaarheid van microRNA-expressie profielen voor classificatie doeleinden te bepalen, werden de resultaten van de microRNA-gebaseerde benadering vergeleken met mRNA-gebaseerde classificatieresultaten. Om te voorkomen dat de selectie van kandidaat-mRNA of -microRNA afhankelijk zou zijn van de samenstelling van de training- en de testset, werden tien verschillende sets van training- en testverbindingen gegenereerd. Daarnaast werd een leaveone-out-gebaseerde voorselectie uitgevoerd om te voorkomen dat de identificatie van kandidaat-mRNA of -microRNA afhankelijk zou zijn van verbindingsspecifieke effecten. Statistisch significante mRNA's (DEGs) en microRNA's (DE-miRs), die overlappend waren tussen de verschillende sets van de training- en testverbindingen, werden vervolgens geïdentificeerd. Met slechts deze specifieke kandidaten werd de voorspellingsanalyse uitgevoerd met behulp van de tien verschillende training- en testsetcomposities, door toepassing van de zogenoemde "nearest-shrunken-centroid"-methode binnen het "Prediction Analysis for Microarrays (PAM)"-programma. Helaas waren we niet in staat om overlappende DE-miRs te identificeren die geschikt waren om GTXC of NGTXC in vivo nauwkeurig te kunnen voorspellen. Daarentegen, in het bijzonder na de 48-urige blootstellingsperiode, zijn twee kandidaat-DEGs geïdentificeerd die in staat zijn in vivo GTX te voorspellen. Een gemiddelde nauwkeurigheid van $93 \%$ (een gevoeligheid van $75 \%$ en een specificiteit van $100 \%$ ) kon worden bereikt voor het voorspellen van de verbindingen in de testset. Tributylinoxide (TBTO), die een onbeslist GTX karakter heeft, werd foutief geclassificeerd. Ook voor het voorspellen van in vivo NGTXC, werd een voorspellend model op basis van mRNA-expressie profielen ontwikkeld. Drie DEGs konden worden geïdentificeerd na 48 uur blootstelling, wat resulteerde in een model dat NGTXC kan voorspellen met een nauwkeurigheid van 88\% (een gevoeligheid van 83\% en een specificiteit van 93\%). Ondanks de relatief hoge nauwkeurigheid en gevoeligheid van het voorspellende model, werd Wy-14643 (Wy) foutief geclassificeerd. De carcinogene potentiaal van Wy in PMH is twijfelachtig in de afwezigheid van naburige Kupffercellen en kan dus worden gemist binnen het momenteel toegepaste in vitro-lever model. Uit de bevindingen in hoofdstuk 4 hebben we geconcludeerd dat de mRNA-gebaseerde voorspelling van GTX en NGTXC beter presteert dan de gelijkwaardige microRNAgebaseerde voorspelling. Met de momenteel gebruikte set van verbindingen, is de microRNAgebaseerde voorspelling van deze eigenschappen niet mogelijk, echter een breder scala van prototypische verbindingen zouden moeten worden onderzocht om deze negatieve bevindingen verder te valideren.

\section{Regulatoire interactie netwerken in relatie tot GTX-blootstelling en geassocieerd met HCC}

Een andere vereiste voor de ontwikkeling van een alternatief in vitro-systeem is dat het relevant is voor de humane in vivo situatie. De beste optie voor een dergelijke robuust testsysteem is het gebruik van primaire humane hepatocyten $(\mathrm{PHH})^{41,42}$. Vaak bestaan er echter inter-individuele verschillen tussen verschillende hepatocyt donoren en daarom is het uiterst ingewikkeld om uit deze resultaten algemene conclusies te trekken die relevant zijn voor humane risicobeoordeling. Een oplossing voor dit probleem is het kweken van hepatocyten van verschillende donoren samen in een petrischaal om een algemenere GTX-respons te genereren die relevanter zou kunnen zijn voor humane chemische risicobeoordeling van carcinogene stoffen. Aflatoxine B1 (AFB1), geproduceerd door Aspergillus schimmels, is een van de meest 
potente GTX-hepatocarcinogenen, die is staat om AFB1-adducten te induceren met bv. DNA en daarbij mutaties te initiëren die uiteindelijk kunnen leiden tot de ontwikkeling van HCC. Minder is bekend over de mogelijke epigenetische veranderingen die GTXC, specifiek AFB1, zouden kunnen veroorzaken in deze regulatoire netwerken, waarbij ook microRNA's een rol spelen die kunnen leiden tot de inductie van HCC. Veranderingen van epigenetische DNAmethylatie patronen (bevestiging of verwijdering van specifieke methylgroepen op het DNA die genexpressie kunnen beïnvloeden) kunnen in combinatie met microRNA-expressie veranderingen kenmerkend zijn voor een bepaald type GTX-blootstelling. Specifiek veranderde regulatoire genen of netwerken kunnen ook worden gebruikt als potentiële kandidaten voor genotoxiciteits- en carcinogeniciteitstesten. In hoofdstuk $\mathbf{5}$ werd een kweekpool van gecryopreserveerde PHH behandeld met twee doses (laag: 0,3 $\mu \mathrm{M}$ en matige: 1 $\mu \mathrm{M}$ ) van de prototypische GTXC-verbinding, aflatoxine B1 (AFB1). Na een vijfdaagse herhaalde blootstelling werden de PHH geoogst en microRNA- en mRNA-expressie veranderingen gemeten tezamen met DNA-methylatie veranderingen in het promotor gebied van de respectievelijke genen. Om de relevantie van de in vitro waargenomen transcriptoom veranderingen, ten opzichte van het risico op HCC in vivo te evalueren, werden de resultaten vergeleken met een kenmerkend genexpressie profiel gebaseerd op data van HCC patiënten ${ }^{43}$. Alsmede werden de resultaten vergeleken met een lijst van bekende AFB1-responsieve genen, verkregen uit de zogeheten "Comparative Toxicogenomics Database (CTD)" opmerkelijke bevinding was dat $\mathrm{PHH}$ blootgesteld gedurende vijf dagen aan 0,3 $\mu \mathrm{M}$ AFB1 meer veranderingen lieten zien op het DNA methylatieniveau in vergelijking met cellen blootgesteld aan $1 \mu \mathrm{M}$ AFB1. In het algemeen werden er meer DNAhypomethylatieveranderingen gemeten als gevolg van AFB1 behandeling dan DNAhypermethylatieveranderingen. Bij het vergelijken van PHH blootgesteld aan $0,3 \mu \mathrm{M}$ en $1 \mathrm{uM}$ AFB1 werd een grote reeks aan genen geïdentificeerd die gemeenschappelijk waren veranderd op het DNA-methylatieniveau tussen de twee doses. Zodra de methylatieresultaten werden gecombineerd met de transcriptoomdata, bleef er een veel kleinere set van veranderde genen over, wat aangeeft dat veranderingen op het DNA-methylatieniveau niet altijd rechtstreeks van invloed zijn op genexpressie niveaus. Toen AFB1-geïnduceerde DEGS, die veranderd waren op het DNA-methylatie niveau, werden gecombineerd met DE-miRs konden HCCgerelateerde biologische processen zoals metabolisme, nuclënezuurmetabolisme, DNA-herstel, celcyclus, apoptose, immuunrespons en ionentransport worden vastgesteld. Specifieke pathways binnen deze AFB1-geïnduceerde biologische processen bleken betrokken bij signaaltransductie cascades. Hierbij konden een aantal microRNA-target genen worden geïdentificeerd bestaande uit ARHGAP35, ILK, PPP2R1A, RB1, TFAP2A, HIST1H4D, RhoA, CDK4 en UBC. Deregulering van deze genen en signaaltransductie cascades zijn belangrijke kenmerken van HCC ${ }^{45}$. De bevindingen uit hoofdstuk $\mathbf{5}$ dragen bij aan een beter begrip van GTX-geïnduceerde veranderingen in regulatoire netwerken die zouden kunnen leiden tot de ontwikkeling van HCC.

\section{Persistentie van de GTX-geïnduceerde veranderingen in de regulatoire netwerken die relevant zijn voor de initiatie van $\mathrm{HCC}$}

Om te concluderen of in vitro-carcinogeen-geïnduceerde veranderingen in regulatoire netwerken relevant zijn voor de in vivo ziektetoestand en daarom bruikbaar zijn voor humane risicobeoordeling, is het essentieel dat deze veranderingen persistent en blijvend onomkeerbaar zijn wanneer de carcinogene behandeling wordt beëindigd. In hoofdstuk $\mathbf{6}$ hebben we daarom onderzocht of de veranderingen op het transcriptoom- en het microRNAoomniveau, die zijn waargenomen in een kweekpool van gecryopreserveerde PHH na een vijfdaagse challenge met $1 \mu \mathrm{M}$ AFB1, blijven bestaan nadat de carcinogene behandeling is gestopt (= uitwasperiode). 
Ook in hoofdstuk 6 en hoofdstuk 7 werden veranderingen in het transcriptoom vergeleken met een signatuur HCC-expressie profiel, verkregen uit de lever transcriptoomgegevens van HCC-patiënten, en een lijst van bekende AFB1-induceerbare genen. Een specifiek regulatoir netwerk, gestuurd door de twee persistent tot expressie gekomen en HCC-gerelateerde microRNA's hsa-miR-34b-5 $\mathrm{p}^{46,47}$ en hsa-miR-222-3 $\mathrm{p}^{25}$, lijkt een rol te spelen in een aantal karakteristieke AFB1-geïnduceerde processen, refererend aan metabolisme veranderingen geassocieerd met kanker en regulatie van genexpressie. Bovendien lijken hsa-miR-34a-5p, hsamiR-96-5p en hsa-miR-30a-3p, die alleen na beëindiging van de $1 \mu \mathrm{M}$ AFB1-behandeling differentieel tot expressie komen, persistent tot expressie gekomen HCC-gerelateerde genen te reguleren. Daarnaast werden persisterende veranderingen in $\mathrm{PHH}$ veroorzaakt door $0,3 \mu \mathrm{M}$ AFB1-behandeling op het methyloom-, microRNAoom- en transcriptoomniveau verder onderzocht in hoofdstuk 7, wanneer de blootstelling aan de carcinogene stof werd beëindigd. $\mathrm{Na}$ blootstelling aan deze lagere AFB1 dosis, konden geen DE-miRs worden geïdentificeerd die nog werden aangetroffen na de driedaagse uitwasperiode. Daarentegen werden, na het combineren van transcriptoomdata met resultaten van DNA-methylatie, diverse persistent veranderde hyper- en hypo-gemethyleerde genen geïdentificeerd, die ook blijvend veranderd waren op het transcriptoomniveau. De zes geïdentificeerde genen, die blijvend hypogemethylateerd en omhoog gereguleerd waren (d.w.z. TXNRD1, PCNA, CCNK, DIAPH3, RAB27A en HIST1H2BF), konden in verband worden gebracht met carcinogene processen. De bevindingen uit hoofdstuk 6 en hoofdstuk 7 kunnen daarom bijdragen aan een beter begrip van blijvende microRNA en epigenetische veranderingen, geïnduceerd na GTX blootstelling, binnen regulatoire netwerken, die voorspellend kunnen zijn voor het uiteindelijke ontstaan van HCC.

\section{Conclusie}

In het algemeen is het doel om de rol van microRNA en epigenetica in transcriptoom veranderingen, geïnduceerd door GTX- en NGTX-verbindingen, in diverse in vitrolevermodellen verder te verhelderen, bereikt. We zijn in staat om robuuste responsen, na blootstelling aan de GTX-verbinding cisplatine (hoofdstuk 2), tussen verschillende in vitromodellen te identificeren. Deze robuuste respons werd hoofdzakelijk bepaald door een complex geconserveerd regulatoir netwerk van 4 TFs. Hiermee wordt het belang van het bestuderen van regulatoire mechanismen in de respons op GTX-blootstelling versterkt. Veranderingen in TF regulatoire netwerken zijn al eerder onderzocht, omdat wordt aangenomen dat deze tijdens de evolutie zijn geconserveerd. Bovendien is er aangetoond dat deze regulatoire netwerken een belangrijke rol spelen bij de reactie op het transcriptoomniveau in HepG2-cellen die werden blootgesteld aan de GTX-verbinding benzo(a)pyreen ${ }^{48,49}$. Deze kennis over de rol van regulatoire netwerken in de chemische carcinogenese uit hoofdstuk 2 is uitgebreid met twee additionele studies waarin meer chemische verbindingen werden gebruikt, om zo de robuuste respons te bestuderen in het in vitro-levermodel PMH. MicroRNA- en mRNA-expressie veranderingen zijn hierbij in beschouwing genomen. In de eerste studie (hoofdstuk 3), waarbij PMH zijn onderzocht na blootstelling aan 3 GTXC- en 3 NGTXCverbindingen, werd vooral mmu-microRNA-503-5p gevonden, die een verschillende rol uitoefent binnen de twee groepen van carcinogenen. In deze studie werden echter voornamelijk verbindingsspecifieke microRNA-veranderingen waargenomen. In de tweede, meer uitgebreide studie (hoofdstuk 4) konden we daarom ook concluderen dat, aangezien de meeste van de microRNA-expressie veranderingen die optreden na kortetermijn-blootstelling verbindingspecifiek zijn, deze niet succesvol gebruikt kunnen worden voor de voorspelling van eigenschappen van verbindingen alsook in in vivo genotoxiciteit of carcinogeniciteitstesten. Deze bevindingen werden bevestigd door in vivo-resultaten, verkregen door Melis en collega's ${ }^{50}$, 
die niet in staat waren om een nauwkeurig onderscheid te maken tussen GTXC- en NGTXCverbindingen, op basis van microRNA-expressie profielen afkomstig van muizen, die alvorens zeven dagen waren blootgesteld. MicroRNA-expressie veranderingen zijn echter van toegevoegde waarde, als het gaat om het bestuderen van het werkingsmechanisme van NGTXC-verbindingen, zoals beschreven in hoofdstuk 3. Uit deze studie werd geconcludeerd dat het onderscheid tussen PMH behandeld met GTX-verbindingen van cellen behandeld met NGTX-verbindingen en NGTXC-behandelde PMH van NC-behandelde cellen, het meest succesvol is wanneer genexpressie profielen worden gebruikt. Relevante discriminerende DEmRNA zijn geïdentificeerd die een duidelijke connectie hadden met GTX- en carcinogeneblootstellingen. MicroRNA-expressie veranderingen zijn niet bruikbaar als voorspellende kandidaten voor in vivo genotoxiciteit- en carcinogeniciteitstesten. De analyses van carcinogeen-geïnduceerde veranderingen op het microRNA-niveau, in combinatie met hun targetgenen, zijn wel erg belangrijk als het gaat om nieuwe inzichten in de biologische mechanismen van bv. NGTX-carcinogenese. Bv. als het gaat om de tumorigene progressie van de celcyclus via mmu-miR-503-5p en cycline D2, die een rol kunnen spelen binnen de ontwikkeling van HCC op de langere termijn. Dit is in overeenstemming met de hypothese dat chemisch-geïnduceerde microRNA-expressiealteraties representatief kunnen zijn voor de optredende veranderingen in $\mathrm{HCC}^{22}$. MicroRNA-expressie veranderingen in combinatie met expressiemodificaties van de targetgenen, weergegeven als microRNA-gestuurde regulatoire netwerken, kunnen derhalve bruikbaar zijn bij de ontwikkeling van zogenoemde AOPs belangrijk binnen de humane risicoanalyse. Uit de studies met PHH (hoofdstuk 5-7) concluderen wij dat specifieke veranderingen op het microRNA-expressieniveau naast transcriptoomveranderingen persistent kunnen zijn wanneer de behandeling met een GTXCverbinding wordt beëindigd (hoofdstuk 6). Dit is voornamelijk het geval in PHH die werden behandeld met $1 \mu \mathrm{M}$ van de model GTXC-verbinding AFB1. Naast veranderingen op het microRNA-niveau, lijken ook persistente DNA-methylatieveranderingen op te treden nadat de $0,3 \mu \mathrm{M}$ AFB1-blootstelling is beëindigd. Hierdoor kunnen ook genexpressie veranderingen van verschillende genen optreden, die kunnen bijdragen aan een patroon kenmerkend voor een bepaalde ziekte, zoals HCC (hoofdstuk 7). Evalueren van genexpressieresultaten met een bepaalde, reeds bestaande signatuurprofiel, gebaseerd op HCC-patiëntendata, is daarbij een cruciale stap. De bevinding dat HCC-gerelateerde persistente veranderingen in vitro optreden in $\mathrm{PHH}$, na beëindiging van de carcingene behandeling op het niveau van het transcriptoom, microRNAoom en/of methyloom, is nieuw in het vakgebied van toxicogenomics. Vooral deze persistente, epigenetisch veranderde genen en microRNA's zijn de meest relevante potentiële biomarker kandidaten voor chemisch-geïnduceerde (hepato-) carcinogenese. Daarom kan deze specifieke mechanistische informatie over deze HCC-gerelateerde, persistente veranderde, epigenetisch- en microRNA-gestuurde regulatoire netwerken, gebruikt worden voor de ontwikkeling van AOP, en daarmee bijdragen aan de toekomstige risicoanalyse van humane carcinogenen.

\section{Beperkingen en toekomstige aanbevelingen}

Veelbelovende resultaten zijn verkregen, die wellicht meer inzicht kunnen verschaffen in de rol van epigenetische- en microRNA-gestuurde regulatoire mechanismen, die op hun beurt van belang zijn voor (hepato-) carcinogenese, geïnduceerd door GTXC- en NGTXC-verbindingen. Deze kennis kan, op de lange termijn, worden gebruikt voor de ontwikkeling van AOPs, die belangrijk zijn in de humane risicobeoordeling van chemische stoffen, geneesmiddelen en voedingsstoffen. Deze mechanistische resultaten, over de rol van microRNA's en epigenetica in de chemische carcinogenese, zijn preliminair en kunnen daarom de basis vormen voor toekomstige studies. Toekomstig onderzoek zou zich vooral moeten richten op het valideren 
van de verkregen resultaten aangaande het gebruik van microRNA-expressieniveau voor voorspellingsdoeleinden, bij voorkeur met behulp van $\mathrm{PHH}$, door het evalueren een groter aantal verbindingen. Dit onderzoek zou dan na moeten gaan of de microRNAexpressiepatronen inderdaad niet waardevol zijn voor voorspellingsdoeleinden. Daarnaast zouden andere methodes kunnen worden toegepast die zich meer richten op een netwerkgebaseerde voorselectie of het positioneren van relevante microRNA's voor het voorspellen van genotoxiciteit en niet-genotoxische carcinogeniteit in vivo. Recentelijk is er een dergelijke methode ontwikkeld voor de rangschikking van microRNA's op basis van hun relevantie in een bepaalde ziekte ${ }^{51}$. Bij voorkeur GTXC-, NGTXC- en NC-verbindingen zouden moeten worden gebruikt en hun verschillende werkingsmechanismen zouden in beschouwing moeten worden genomen, bv. voor read-across initiatieven, zoals bij zogenoemde "connectivity mapping"-methode ${ }^{52,53}$. Voorts dient persistentie van de verkregen effecten te worden bekeken, omdat deze bijzonder relevant is voor de ontwikkeling van HCC $^{21,54}$. Daarom zouden er goed ontworpen tijdreeks studies moeten worden uitgevoerd, die zich richten op de reeks van gebeurtenissen die plaatsvinden na carcinogene behandeling. Op deze manier kan informatie worden verkregen, met betrekking to de reactie tijd van microRNA's in PHH, na blootstelling aan verschillende GTXC- en NGTXC-verbindingen. Bovendien moet de invloed van de microRNA-target gen-interacties worden bevestigd op het eiwitniveau, speciek in PHH, om zo de daadwerkelijke invloed van de microRNA-suppressie op genexpressieniveau te meten ${ }^{55}$. Daarnaast zullen de resultaten die in PHH verkregen zijn, moeten worden uitgebreid met meer verbindingen, van verschillende toxiciteitsklassen, om zo meer informatie te genereren met betrekking tot de epigenetische- en microRNA-gestuurde regulatoire netwerken, die zijn veranderd na blootstelling aan GTXC- of NGTXCverbindingen. Om de epigenetische mechanismen volledig te dekken, zullen ook histonacetyleringsveranderingen moeten worden gemeten. Specifieke, kenmerkende epigenetische- en microRNA-gestuurde genexpressie profielen kunnen worden verkregen, die vervolgens de basis kunnen vormen voor de ontwikkeling van mechanistisch-gebaseerde AOPs. Daarom moeten de verkregen resultaten worden gekoppeld aan toxicologische eindpunten (d.w.z. fenotypische verankering) ${ }^{56}$, die kenmerkend zijn voor een bepaalde klasse van verbindingen, zoals bv. DNA-adduct vorming of de aanwezigheid van zuurstof radicalen. Bovendien moeten meer hepatocyt-donoren worden gebruikt, die de verkregen resultaten verder zouden kunnen ondersteunen en die bovendien meer representatief zouden kunnen zijn voor de humane situatie. Andere alternatieve systemen, zoals driedimensionale organotypische lever-organoïde en -sferoïde ${ }^{57}$ celkweeksystemen zoals bv. pluripotente stamcellen afgeleide hepatocytes $^{58}$ of hepatocyt co-culturen zouden kunnen worden gebruikt, die meer representatief zijn voor de humane in vivo lever ${ }^{59}$. Deze driedimensionale lever-organoïde/sferoïde celculturen worden geprefereerd, gezien hun herstelde extracellulaire matrix, en daardoor verbeterde hepatocyt-celpolariteit. Door het herstel van deze kenmerken van de lever lijken de cellen hun metabolische competentie te behouden en kunnen daarom gedurende een langere periode worden gekweekt ${ }^{59}$. Vooral de co-culturen van humane hepatocyten met bv. Kupffercellen (macrofagen), sinusoïdale endotheelcellen en stellaatcellen (stromale cellen) blijken succesvol in het nabootsen van de in vivo humane situatie ${ }^{59}$. Een dergelijk cocultuurmodel van hepatocyten, die in kweek kan worden gehouden voor een langere periode, kan verder helpen bij het bestuderen van de waargenomen effecten van persistente carcinogene blootstelling.

De huidige bevindingen van dit proefschrift, in combinatie met additionele cross-omics gebaseerde toekomstige experimenten, kunnen nieuwe inzichten verschaffen in de regulatoire netwerken, die bijdragen aan het werkingsmechanismse van carcinogene chemische stoffen, welke een voorspellende waarde zouden kunnen geven bij de humane risicobeoordeling. 


\section{$\underline{\text { Referenties }}$}

1. Ferlay, J. et al. Cancer incidence and mortality worldwide: sources, methods and major patterns in GLOBOCAN 2012. Int J Cancer 136, E359-86 (2015).

2. Kew, M.C. Aflatoxins as a cause of hepatocellular carcinoma. J Gastrointestin Liver Dis 22, 305-10 (2013).

3. Ashby, J. Use of short-term tests in determining the genotoxicity or nongenotoxicity of chemicals. IARC Sci Publ, 135-64 (1992).

4. Silva Lima, B. \& Van der Laan, J.W. Mechanisms of nongenotoxic carcinogenesis and assessment of the human hazard. Regul Toxicol Pharmacol 32, 135-43 (2000).

5. Ames, B.N., Lee, F.D. \& Durston, W.E. An improved bacterial test system for the detection and classification of mutagens and carcinogens. Proc Natl Acad Sci US A 70, 782-6 (1973).

6. Kirkland, D., Reeve, L., Gatehouse, D. \& Vanparys, P. A core in vitro genotoxicity battery comprising the Ames test plus the in vitro micronucleus test is sufficient to detect rodent carcinogens and in vivo genotoxins. Mutat Res 721, 27-73 (2011).

7. Matsushima, T. et al. Validation study of the in vitro micronucleus test in a Chinese hamster lung cell line (CHL/IU). Mutagenesis 14, 569-80 (1999).

8.

Moller, P. Genotoxicity of environmental agents assessed by the alkaline comet assay. Basic Clin Pharmacol Toxicol 96 Suppl 1, 1-42 (2005).

9. Shelby, M.D. \& Witt, K.L. Comparison of results from mouse bone marrow chromosome aberration and micronucleus tests. Environ Mol Mutagen 25, 302-13 (1995).

10. Kirkland, D. et al. How to reduce false positive results when undertaking in vitro genotoxicity testing and thus avoid unnecessary follow-up animal tests: Report of an ECVAM Workshop. Mutat Res 628, 31-55 (2007).

11. Hernandez, L.G., van Steeg, H., Luijten, M. \& van Benthem, J. Mechanisms of non-genotoxic carcinogens and importance of a weight of evidence approach. Mutat Res 682, 94-109 (2009).

12. Ennever, F.K. \& Lave, L.B. Implications of the lack of accuracy of the lifetime rodent bioassay for predicting human carcinogenicity. Regul Toxicol Pharmacol 38, 52-7 (2003).

13. Kirkland, D. Improvements in the reliability of in vitro genotoxicity testing. Expert Opin Drug Metab Toxicol 7, 1513-20 (2011).

14. Kitano, H. Computational systems biology. Nature 420, 206-10 (2002).

15. Kleinjans, J. Toxicogenomics-based cellular models : alternatives to animal testing for safety assessment, xviii, 348 p. (Elsevier/AP, Amsterdam, 2014).

16. Piersma, A.H. et al. A critical appraisal of the process of regulatory implementation of novel in vivo and in vitro methods for chemical hazard and risk assessment. Crit Rev Toxicol 44, 876-94 (2014).

17. Ankley, G.T. et al. Adverse outcome pathways: a conceptual framework to support ecotoxicology research and risk assessment. Environ Toxicol Chem 29, 730-41 (2010).

18. National Research Council (U.S.). Committee on Applications of Toxicogenomic Technologies to Predictive Toxicology. Applications of toxicogenomic technologies to predictive toxicology and risk assessment, xxii, 275 p. (National Academies Press, Washington, D.C., 2007).

19. Olden, K. Toxicogenomics--a new systems toxicology approach to understanding of gene-environment interactions. Ann N Y Acad Sci 1076, 703-6 (2006).

20. Goodman, J.I. et al. What do we need to know prior to thinking about incorporating an epigenetic evaluation into safety assessments? Toxicol Sci 116, 375-81 (2010).

21. Mirbahai, L. \& Chipman, J.K. Epigenetic memory of environmental organisms: a reflection of lifetime stressor exposures. Mutat Res Genet Toxicol Environ Mutagen 764-765, 10-7 (2014).

22. Izzotti, A. \& Pulliero, A. The effects of environmental chemical carcinogens on the microRNA machinery. International Journal of Hygiene and Environmental Health 217, 601-627 (2014).

23. Giordano, S. \& Columbano, A. MicroRNA's: new tools for diagnosis, prognosis, and therapy in hepatocellular carcinoma? Hepatology 57, 840-7 (2013).

24. Gramantieri, L. et al. MicroRNA involvement in hepatocellular carcinoma. J Cell Mol Med 12, 2189-204 (2008).

25. Karakatsanis, A. et al. Expression of microRNA's, miR-21, miR-31, miR-122, miR-145, miR-146a, miR200c, miR-221, miR-222, and miR-223 in patients with hepatocellular carcinoma or intrahepatic cholangiocarcinoma and its prognostic significance. Mol Carcinog 52, 297-303 (2013).

26. Li, J., Wang, Y., Yu, W., Chen, J. \& Luo, J. Expression of serum miR-221 in human hepatocellular carcinoma and its prognostic significance. Biochem Biophys Res Commun 406, 70-3 (2011).

27. Pineau, P. et al. miR-221 overexpression contributes to liver tumorigenesis. Proc Natl Acad Sci U S A 107, 264-9 (2010).

28. Sun, J., Lu, H., Wang, X. \& Jin, H. MicroRNA's in hepatocellular carcinoma: regulation, function, and clinical implications. ScientificW orldJournal 2013, 924206 (2013). 
29. Xiong, Y. et al. Effects of microRNA-29 on apoptosis, tumorigenicity, and prognosis of hepatocellular carcinoma. Hepatology 51, 836-45 (2010).

30. Baylin, S.B., Herman, J.G., Graff, J.R., Vertino, P.M. \& Issa, J.P. Alterations in DNA methylation: a fundamental aspect of neoplasia. Adv Cancer Res 72, 141-96 (1998).

31. Bird, A. DNA methylation patterns and epigenetic memory. Genes Dev 16, 6-21 (2002).

32. Bollati, V. et al. Exposure to metal-rich particulate matter modifies the expression of candidate microRNA's in peripheral blood leukocytes. Environ Health Perspect 118, 763-8 (2010).

33. Cheng, Y. et al. Correlation of $\mathrm{CpG}$ island methylator phenotype with poor prognosis in hepatocellular carcinoma. Exp Mol Pathol 88, 112-7 (2010).

34. Hernandez-Vargas, H. et al. Hepatocellular Carcinoma Displays Distinct DNA Methylation Signatures with Potential as Clinical Predictors. PLoS ONE 5, e9749 (2010).

35. Lee, Y.W. et al. Carcinogenic nickel silences gene expression by chromatin condensation and DNA methylation: a new model for epigenetic carcinogens. Mol Cell Biol 15, 2547-57 (1995).

36. Shen, J. et al. Genome-wide aberrant DNA methylation of microRNA host genes in hepatocellular carcinoma. Epigenetics 7, 1230-7 (2012).

37. Zhang, Y.J. et al. Global hypomethylation in hepatocellular carcinoma and its relationship to aflatoxin B(1) exposure. World J Hepatol 4, 169-75 (2012).

38. Osella, M., Riba, A., Testori, A., Cora, D. \& Caselle, M. Interplay of microRNA and epigenetic regulation in the human regulatory network. Front Genet 5, 345 (2014).

39. Rieswijk, L., Lizarraga, D., Brauers, K.J., Kleinjans, J.C. \& van Delft, J.H. Characterisation of cisplatininduced transcriptomics responses in primary mouse hepatocytes, HepG2 cells and mouse embryonic stem cells shows conservation of regulating transcription factor networks. Mutagenesis 29, 17-26 (2014).

40. Rieswijk, L. et al. Evaluating microRNA profiles reveals discriminative responses following genotoxic or non-genotoxic carcinogen exposure in primary mouse hepatocytes. Mutagenesis (2015).

41. Lecluyse, E.L. \& Alexandre, E. Isolation and culture of primary hepatocytes from resected human liver tissue. Methods Mol Biol 640, 57-82 (2010).

42. Hewitt, N.J. et al. Primary hepatocytes: current understanding of the regulation of metabolic enzymes and transporter proteins, and pharmaceutical practice for the use of hepatocytes in metabolism, enzyme induction, transporter, clearance, and hepatotoxicity studies. Drug Metab Rev 39, 159-234 (2007).

43. Caiment, F., Tsamou, M., Jennen, D. \& Kleinjans, J. Assessing compound carcinogenicity in vitro using connectivity mapping. Carcinogenesis 35, 201-7 (2014).

44. Davis, A.P. et al. The Comparative Toxicogenomics Database: update 2013. Nucleic Acids Res 41, D1104-14 (2013).

45. Hermeking, H. The miR-34 family in cancer and apoptosis. Cell Death Differ 17, 193-9 (2010).

$\mathrm{Xu}$, Y. et al. A potentially functional polymorphism in the promoter region of miR-34b/c is associated with an increased risk for primary hepatocellular carcinoma. Int J Cancer 128, $412-7$ (2011).

49. $\quad$ vactors: function, expression and evolution. Nat Rev Genet 10, 252-63 (2009). network of transcription factors regulates the effects on functional gene sets. Toxicol Sci 117, 381-92 (2010).

50. Melis, J.P. et al. In vivo murine hepatic microRNA and mRNA expression signatures predicting the (non)genotoxic carcinogenic potential of chemicals. Arch Toxicol (2014).

Le, D.H. Network-based ranking methods for prediction of novel disease associated microRNA's. Comput Biol Chem 58, 139-148 (2015).

Caiment, F., Tsamou, M., Jennen, D. \& Kleinjans, J. Assessing compound carcinogenicity in vitro using connectivity mapping. Carcinogenesis Advance Access published August 12, 2013, doi:10.1093/carcin/bgt278 (2013).

Lamb, J. et al. The Connectivity Map: using gene-expression signatures to connect small molecules, genes, and disease. Science 313, 1929-35 (2006).

Heijmans, B.T. et al. Persistent epigenetic differences associated with prenatal exposure to famine in humans. Proc Natl Acad Sci US A 105, 17046-9 (2008).

Selbach, M. et al. Widespread changes in protein synthesis induced by microRNA's. Nature 455, 58-63 (2008).

Waters, M.D. \& Fostel, J.M. Toxicogenomics and systems toxicology: aims and prospects. Nat Rev Genet 5 , 936-948 (2004).

Hynds, R.E. \& Giangreco, A. Concise review: the relevance of human stem cell-derived organoid models for epithelial translational medicine. Stem Cells 31, 417-22 (2013).

59. Soldatow, V.Y., LeCluyse, E.L., Griffith, L.G. \& Rusyn, I. In vitro models for liver toxicity testing. Toxicology research 2, 23-39 (2013). 
C



0

O



00200

$\mathrm{O}$

() Valorization

O

O

0

C O

O

O

O

00

O

()

○

()

0

()

)

)

c

0

00

00

()

000

○

0

()

O 


\section{Valorization}

\section{Relevance}

The overall aim of this thesis was to investigate what regulatory roles microRNAs and epigenetic changes (i.e. DNA methylation) have in transcriptomic responses induced by genotoxic and non-genotoxic carcinogens in cell models relevant for liver carcinogenicity. Furthermore, the application of the expression patterns of these regulatory molecules (i.e. microRNAs and DNA methylation changes), for the purpose of discriminating between different classes of chemical compounds (i.e. genotoxic carcinogens (GTXC), non-genotoxic carcinogens (NGTXC) and non-carcinogens (NC)), was evaluated in the context of genotoxicity and carcinogenicity testing. Cancer is the number one cause of death worldwide ${ }^{1}$. According to the World Health Organization, liver cancer, specifically hepatocellular carcinoma (HCC) is the sixth most prevalent and the second most lethal type of cancer worldwide 2 . Exposure to hazardous agents (e.g. chemicals, food-additives or pharmaceuticals) appears to be of major influence in the induction of human cancers. For this reason, it is extremely important that the carcinogenic potential of new agents is accurately predicted. Traditionally, carcinogens are divided based on their mode of action (MOA) into DNAreactive genotoxic (GTX) carcinogens and non DNA-reactive or non-genotoxic (NGTX) carcinogens $^{3}$. Avoidance of exposure to such carcinogens may prevent $30 \%$ of all HCC cases ${ }^{4}$. The safe use of these agents and accurate testing of their toxic effects is of economical as well as societal concern.

Valorization of scientific knowledge is defined as the process of value-creation out of knowledge, by making this knowledge suitable and available for economic or societal utilization and to translate this into high-potential products, services, processes and industrial activity.

\section{Economic relevance}

Economic utilization of the current obtained scientific knowledge should be focused on developing alternative, improved and less expensive in vitro tests which are able to better predict the in vivo genotoxic and carcinogenic potential of novel hazardous agents (e.g. chemicals, food-additives or pharmaceuticals). Development, application and commercialization of improved test methods in human risk assessment by industry leads to products which are safer leading to a higher competitive value and are thus more profitable.

\section{Societal relevance}

Societal utilization of the scientific knowledge originating from this current thesis should be dedicated to follow-up research focused on the development of so called adverse outcome pathways (AOP) by the global organization, Organization for Economic Co-operation and Development (OECD) $)^{5}$. The OECD actually launched a program in 2012 on the development of AOPs that should contribute to an improved risk assessment of human carcinogens. The definition of an AOP, as meant by the OECD, is an analytical concept that describes a linear sequence of causally related key events (KE) within the different molecular layers of the biological organization; starting with a so called molecular initiation event (MIE) that ultimately lead to an adverse outcome (AO), represented by a health or toxicological effect such as the development of HCC. The linkage between the events is described by key event relationships (KER). Ideally these AOPs, and information on these related events, should all be collected 
within an open-source web-based interface, namely the AOP Knowledge Base (AOP KB) (https://aopkb.org/). The project focused on collecting this type of information is led by the OECD, the U.S. Environmental Protection Agency (EPA) and the European Commission's Joint Research Center (JRC). AOPs are the central element of a toxicological knowledge framework being built to support chemical risk assessment based on mechanistic reasoning. Such AOPs should also be included in the guidance documents developed by an initiative of the European Union through the Registration, Evaluation, Authorization and restrictions of Chemicals $(\mathrm{REACH})^{6}$.

In addition the generation of improved novel alternative in vitro test methods could on the long run, replace the use of animals for genotoxicity and carcinogenicity testing, within human risk assessment, thereby complying with the societal demand for reducing animal-based testing.

\section{Target groups}

\section{Industry}

The mechanistic knowledge obtained in this current thesis might be used within future translational research aimed at developing in vitro applications that accurately predict the carcinogenic potential of a novel agent developed by chemical, food or pharmaceutical industry. Especially specific response profiles of genes or other biomarkers may be utilized for developing such alternative test systems. This may fulfill the economic demand of creating products which are safer to use thereby generating more competitive products.

\section{Regulatory organizations}

In Europe, specialized organizations such as the European Chemical Association (ECHA) European Food Safety Authority (EFSA) ${ }^{7}$ and the European Medicines Agency/U.S. Food and Drug Administration (EMA/FDA) ${ }^{8,9}$ are responsible for the safe use of chemicals, foodadditives and pharmaceuticals, respectively. Regulations and recommendations are provided by global initiatives such as the guidelines developed by the $\mathrm{OECD}^{5}$ or by the European Union through $\mathrm{REACH}^{6}$.

New chemicals, food-additives or drugs first need to be extensively tested for their potential hazards and risks to human health and the environment, before they enter the public market. Human risk assessment is based on toxicological tests performed with short-term in vitro assays and (sub-) chronic animal experiments. The animal experiments, mainly using mice and rats, are used to estimate the human cancer risks, but are very expensive and time consuming as they require many animals and a large amount of test compound during a period of usually 2 years. The effectiveness, reliability and relevance of these in vivo animal experiments are questioned, especially with regards to the extrapolation of data from animal to humans and from short-term experiments in animals to long-term real-life exposure in $\operatorname{man}^{10}$. Due to inconsistencies within the translation of testing results from rodents to humans, certain undetected carcinogenic compounds may enter the market while other compounds are incorrectly withdrawn from the market due to their exclusive carcinogenic potential in rodents ${ }^{11}$.

Withdrawal of a novel agent from the public market may have tremendous financial consequences for industry. Therefore, the demand for alternative testing methods, for better predicting human cancer risks, is increasing and new high throughput in vitro methods need to 
be developed. Numerous relatively cheap in vitro tests are available (e.g. bacterial Ames test, mouse lymphoma test, micronucleus test and the chromosomal aberration test) to determine if a compound or drug has carcinogenic potential, however these are characterized by a high false positive rate (falsely predicted to be carcinogenic when compared with in vivo data) ${ }^{12,13}$. Many compounds that are tested positively in vitro therefore need additional animal tests, which thus may be unnecessary. Further development of reliable standardized in vitro tests is therefore requested by the OECD and REACH as well as industry which correctly predict human cancer risks ${ }^{6}$. The focus of the development of these alternative models may very well be on liverbased systems. The liver is responsible for the processing of chemical compounds to potential carcinogens and is also a major target organ. In the last decade the development of new alternative test systems has focused on the application of novel technologies, thereby enabling complete monitoring of the effects of carcinogens in a cell which allows us to better understand why these compounds cause cancer in the long run. The field of toxicogenomics, in which this so called systems biology approach may be applied, can be extremely helpful in improving the translation of the in vitro setting to the in vivo situation ${ }^{14-16}$.

Furthermore, toxicogenomics-based alternative in vitro assays may help in a better understanding of the regulatory mechanisms (e.g. epigenetic events such as DNA methylation or via non-coding microRNAs) underlying the carcinogenic potential of certain types of agents. The findings from Chapter 3 might be useful in developing a cross-omics based AOP for GTXC versus NGTXC, in which the roles of microRNAs in chemically-induced carcinogenesis leading towards HCC may provide an added value. Especially the findings, presented in Chapter 5, 6 and 7, regarding the persistent effects on the microRNA and gene expression and DNA methylation level after, in this case, termination of AFB1 treatment in primary human hepatocytes $(\mathrm{PHH})$ may add a new perspective on AOPs for carcinogenicity in particular and toxicity in general. An increased understanding of chemically-induced carcinogenesis and especially the persistency of the induced effects due to carcinogen treatment, may lead towards an improved risk assessment of chemical carcinogens within humans by contributing to a controlled regulatory environment in which the safe use of products and processes is guaranteed. This may ultimately lead to an improvement of health of patients and consumers.

The development of AOPs may therefore facilitate in a better prediction of for example the onset of HCC ${ }^{17}$. In the AOP Knowledge Base wiki (https://aopkb.org/aopwiki/index.php/Main Page) there is currently an AOP under review named "AFB1: Mutagenic Mode-of-Action leading to Hepatocellular Carcinoma (HCC)" developed by the working group of OECD Project 2.8 focused on developing AOPs for Mutagenic Modes of Action for Cancer. Our obtained regulatory networks could provide added value to this AOP and may therefore be uploaded to the AOP Knowledge Base wiki and reviewed by the OECD. The epigenetic- and microRNA-directed regulatory networks obtained within this current study might therefore be used for further development of AOPs characteristic for a particular type of exposure but could also be useful in generating AOPs representative for more general responses (GTX versus NGTX carcinogen exposure) ${ }^{18}$.

\section{Activities or products}

\section{Commercialized in vitro test system}

mRNA expression-based in vivo and in vitro tests have already proven to be successful in separating, specifically, human cells or rats treated with GTX from NGTX carcinogens 19-21. 
The gene expression found within Chapter 4 could therefore be used for the development of an alternative in vitro test system which could be detected by a dedicated PCR- or microarraybased test system within PMH. Such an alternative test system should then be accompanied with a standard operating procedure (SOP) concerning: 1) how to effectively culture PMH and 2) how to design a sophisticated experiment. This concept could then be commercially sold as a quick and standardized ready-to-go test system for the evaluation of carcinogenic properties of novel chemical compounds.

\section{Adverse Outcome Pathways (AOP)}

AOPs might be developed representing a set of characteristic responsive molecules and their behavior or expression within a cell, in response to a given exposure with a particular compound. By defining particular classes of toxicity, using prototypical model compounds, chemical AOPs may be established which can be used for a better mechanistic understanding and prediction of toxicity of new compounds, food-additives or pharmaceuticals. Generation of AOPs representing important events in genotoxicity and carcinogenicity should be included in regulatory guidance documents for the testing of novel agents. Therefore, the obtained persistently affected genes (non-methylated/methylated and/or differentially up/down regulated) and microRNAs, which were found in this current thesis after termination of AFB1 treatment in $\mathrm{PHH}$ (Chapter $5-7$ ), could be form the basis for the development of such an AOP.

\section{Innovation}

\section{Dedicated gene expression platforms}

The main innovative value of the obtained results is to be found in the applicability of a gene expression pattern, more specifically the differentially expressed genes, within an commercialized test system which may improve the in vitro-based prediction of in vivo genotoxicity and carcinogenicity. In Chapter 4 we initially aimed at developing a similar test system which was preferably based on microRNA expression profiles; however, we were not successful. Instead, we were able to establish such a predictive signature for genotoxicity and, specifically, non-genotoxic carcinogenicity which was based on gene expression profiles. Such an expression profile based on mRNA data has already been proven successful in predicting in vivo genotoxicity with the use of in vitro cultured HepG2 cells by Magkoufopoulou and colleagues ${ }^{19}$. This in vitro-based expression profile has been patented and licensed out to the spin off company ToxGenSolutions (http://www.toxgensolutions.eu/) to develop a commercialized PCR-based platform. A comparable platform may thus be developed focused on an improved prediction of in vivo genotoxicity or non-genotoxic carcinogenicity. Our robust gene expression profile could be applied which is based on in vitro transcriptomics data originating from exposed primary mouse hepatocytes. The availability of a more accurate in vitro test system may greatly benefit big partners in industry since such a method would lead to the production of safer and more competitive products with a higher market value.

\section{Improvement of mechanistic understanding}

Furthermore, within this thesis we were able to establish the basis for the development of AOPs, important within genotoxicity and carcinogenicity related events, using a set of prototypical model compounds. These AOPs could improve the current understanding of 
how chemicals provoke toxic events leading to a pathological outcome. Therefore the results of this thesis improve the current knowledge with regards to mechanism of toxicity.

\section{Implementation}

The development of an appropriate AOP for chemically-induced carcinogenesis could improve the mechanistic understanding of toxicological endpoints related with certain pathologies. Replacing animal experiments with an improved and more mechanistically relevant alternative test system may generate major societal as well as economic impact. Furthermore, better insights into carcinogenic mechanisms might lead to more efficient and cost-effective future experiments.

\section{Economic implementation}

Therefore, in order to further validate the use of potential biomarker genes or microRNAs for the development of an alternative in vitro test system, the current results from this thesis need to be further expanded with more test chemicals. Also for the development of potential biomarkers of genotoxicity or carcinogenicity this is relevant in order to further develop the training set of compounds. The training set of compounds is used to establish the chemical specific biomarkers. When the training set is sufficiently large and accurate biomarkers are established, the tests needs to be reviewed and validated by an independent laboratory with a group of test compounds. The data from these extended experiments then need to be used within appropriate prediction software programs that are able to accurately predict the toxic potential of the tested compound. For achieving economic impact, preferably, the biomarkers need to be patented. This may be commercialized by developing a standardized dedicated analytical platform as well as software for the analysis of the data.

\section{Societal implementation}

Furthermore, in order to get the new test generally accepted in chemical safety assessment, recognition by regulatory authorities is required. Validation of this new method therefore needs to be reviewed by the European Centre for the Validation of Alternative Methods $(\text { ECVAM })^{22}$ in order to evaluate the reproducibility and the robustness of this assay. When the new tests finds acceptance by ECVAM it may be implemented as an EU test method and included in the EU Test Methods Regulation or as an OECD Test Guideline ${ }^{5}$. The OECD Test Guidelines are a collection of internationally agreed test methods used by government, industry and independent laboratories. They are used to determine the safety of chemicals and chemical preparations, including pesticides and industrial chemicals.

Other ways of valorization by then also include instructing and informing personal from governmental institutes, industry and independent laboratories about these new guidelines in order to allow correct application of the new test method.

The results from this thesis may form the preliminary basis for developing such AOP- or biomarker-focused alternative tests systems thereby contributing to a more accurate prediction of carcinogenicity for human risk assessment. 


\section{References}

1. Stewart, B.W., Wild, C., International Agency for Research on Cancer \& World Health Organization. World cancer report 2014, xiv, 630 pages (International Agency for Research on Cancer; WHO Press, Lyon, France; Geneva, Switzerland, 2014).

2. Ferlay, J. et al. Cancer incidence and mortality worldwide: sources, methods and major patterns in GLOBOCAN 2012. Int J Cancer 136, E359-86 (2015).

3. Ashby, J. Use of short-term tests in determining the genotoxicity or nongenotoxicity of chemicals. $I A R C$ Sci Publ, 135-64 (1992).

4. World Health Organization. National cancer control programmes : policies and managerial guidelines, xxiii, $180 \mathrm{p}$. (World Health Organization, Geneva, 2002).

5. Organisation for Economic Co-operation and Development (OECD). OECD Guidelines for the Testing of Chemicals http://www.oecd.org/chemicalsafety/testing/oecdguidelinesforthetestingofchemicals.htm, (accessed on July 15, 2015) (2015).

6. European Chemicals Agency (ECHA). Registration, Evaluation, Authorisation and Restriction of Chemicals (REACH). http://echa.europa.eu/regulations/reach, (accessed on July 15, 2015) (2015). European Food Safety Authority (EFSA). http://www.efsa.europa.eu/, (accessed on July 15, 2015) (2015). European Medicines Agency (EMA). http://www.ema.europa.eu/ema/, (accessed on July 15, 2015) (2015).

9. U.S. Food and Drug Administration (FDA). http://www.fda.gov/, (accessed on July 15, 2015) (2015).

10. Walmsley, R.M. \& Billinton, N. How accurate is in vitro prediction of carcinogenicity? British Journal of Pharmacology 162, 1250-1258 (2011).

11. Ennever, F.K. \& Lave, L.B. Implications of the lack of accuracy of the lifetime rodent bioassay for predicting human carcinogenicity. Regul Toxicol Pharmacol 38, 52-7 (2003).

12. Kirkland, D. \& Speit, G. Evaluation of the ability of a battery of three in vitro genotoxicity tests to discriminate rodent carcinogens and non-carcinogens III. Appropriate follow-up testing in vivo. Mutat Res 654, 114-32 (2008).

13. Kirkland, D. et al. How to reduce false positive results when undertaking in vitro genotoxicity testing and thus avoid unnecessary follow-up animal tests: Report of an ECVAM Workshop. Mutat Res 628, 31-55 (2007).

14. National Research Council (U.S.). Committee on Applications of Toxicogenomic Technologies to Predictive Toxicology. Applications of toxicogenomic technologies to predictive toxicology and risk assessment, xxii, 275 p. (National Academies Press, Washington, D.C., 2007).

15. Kleinjans, J. Toxicogenomics-based cellular models : alternatives to animal testing for safety assessment, xviii, $348 \mathrm{p}$. (Elsevier/AP, Amsterdam, 2014).

16. Piersma, A.H. et al. A critical appraisal of the process of regulatory implementation of novel in vivo and in vitro methods for chemical hazard and risk assessment. Crit Rev Toxicol 44, 876-94 (2014).

17. Ankley, G.T. et al. Adverse outcome pathways: a conceptual framework to support ecotoxicology research and risk assessment. Environ Toxicol Chem 29, 730-41 (2010).

18. Mirbahai, L. \& Chipman, J.K. Epigenetic memory of environmental organisms: a reflection of lifetime stressor exposures. Mutat Res Genet Toxicol Environ Mutagen 764-765, 10-7 (2014).

19. Magkoufopoulou, C. et al. A transcriptomics-based in vitro assay for predicting chemical genotoxicity in vivo. Carcinogenesis (2012).

20. Fielden, M.R. et al. Development and evaluation of a genomic signature for the prediction and mechanistic assessment of nongenotoxic hepatocarcinogens in the rat. Toxicol Sci 124, 54-74 (2011).

21. Ellinger-Ziegelbauer, H., Stuart, B., Wahle, B., Bomann, W. \& Ahr, H.J. Comparison of the expression profiles induced by genotoxic and nongenotoxic carcinogens in rat liver. Mutat Res 575, 61-84 (2005).

22. European Union Reference Laboratory for Alternatives to Animal Testing (EURL-ECVAM). https://eurlecvam.jrc.ec.europa.eu/, (accessed on July 15, 2015) (2015). 
C

00

0

O

Agdendum III

00000

0 D Dankwwoord

O

$\bigcirc$

$0(0$

() $\mathrm{O}$

0

○

○

○

00

○

()

()

0

Q

$0 \quad 0$

C)

O

()

O

00

00

()

000

O

0

() 


\section{Dankwoord}

Nou daar zit ik dan, met de laatste puntjes op de $\mathrm{i}$ is er nu toch echt een einde gekomen aan mijn promotietraject. En wat voor een. Ik denk wel dat ik kan zeggen dat ik alle aspecten van een $\mathrm{PhD}$ heb mogen ervaren; inclusief de ups en downs. Al met al ligt er nu wel een mooi afgerond proefschrift op tafel en mag ik trots zijn dat ik dit heb voltooid. Ik had dit natuurlijk nooit kunnen afmaken zonder de hulp van een grote groep van mensen en met behulp van dit dankwoord wil ik die mensen dan ook in het bijzonder bedanken.

Allereerst natuurlijk het promotie team. Een uiterst dynamisch team kan ik wel stellen. Waar ik mijn promotietraject begon met een promotor, een copromotor en een begeleider, eindig ik met eenzelfde promotor maar met een totaal andere copromotor.

Ten eerste wil ik graag professor Kleinjans bedanken. Jos, de eerste paar jaar van mijn promotietraject was ons contact erg sporadisch. Dit veranderde echter na het plotselinge vertrek van zowel mijn begeleider als copromotor tijdens het derde jaar van mijn promotietraject. Ik wil je graag bedanken voor het feit dat je, hoe druk je het ook had, altijd wel tijd wist vrij te maken om opbouwende en doelgerichte kritiek op mijn manuscripten te geven. Bovendien wist je veelvuldig effectief te snijden in mijn veelal ellenlange verhalen. Bedankt ook voor de vele discussies die we in het laatste jaar hebben gevoerd omtrent de uitvoering van mijn experimenten en het schrijven van de nodige papers. Waar ik af en toe nog wel eens wat pessimistisch uit de hoek kon komen wist jij altijd wel het positieve te benadrukken. Even leek het af en toe alsof er helemaal niets meer goed kon gaan, maar gelukkig hebben we met de nodige vakkundigheid en bovendien een doelgerichte aanpak het geheel op een goede manier kunnen afronden.

Ten tweede wil ik ook graag mijn copromotor, Dr. van Breda, bedanken. Simone, ontzettend bedankt dat je in het laatste jaar hebt besloten om mij in deze kritieke fase te begeleiden. Specifiek in de fase waarin ik het helemaal niet meer zag zitten, kon ik gelukkig bij jou terecht om mijn hart te luchten. Mede dankzij jouw stap voor stap benadering hebben we stukje bij beetje alles weten af te ronden. Bedankt ook voor jouw expertise op het gebied van DNA methylatie analyses en je input bij de lastige experimenten met de humane hepatocyten. Mede dankzij jouw hulp hebben we dit allemaal tot een goed einde kunnen brengen en heb ik daar uiteindelijk drie hoofdstukken aan kunnen wijden.

Zoals gezegd begon ik mijn promotietraject met een andere copromotor. Hij hoort officieel dan ook niet meer bij mijn promotieteam maar bij deze wil ik ook Dr. van Delft bedanken. Joost, dankjewel voor de nodige discussies aan het begin van mijn promotietraject betreffende nieuwe experimenten en de nodige analyses. Alhoewel je op dit moment niet meer werkzaam bent binnen de wetenschap wil ik je wel graag bedanken voor de kans die je mij hebt gegeven om dit promotietraject te mogen volgen. Ik wens je heel veel succes met je nieuwe loopbaan binnen de ouderenzorg in het Verenigd Koninkrijk.

Ook wil ik graag Dr. Lizarraga bedanken voor het begeleiden van mijn promotietraject. Thanks Daneida for your critical view on new experiments and analyses and your help with the mouse hepatocytes studies. Without your help I could never have done these analyses and two chapters of my thesis would not have been there. I hope that it has been fruitful starting a new life with your daughter and husband in the U.S. 
Uiteraard wil ik ook graag mijn beoordelingscommissie bedanken, bestaande uit de voorzitter Prof. dr. E.C.M. Mariman en de overige leden Prof. dr. H. Van Loveren, Dr. H. Vrieling en Prof. dr. B. van de Water voor het evalueren van mijn proefschrift en hun bereidwilligheid om zitting te nemen in de corona tijdens de openbare verdediging van mijn promotieonderzoek. In het kader van de oppositie wil ik ook graag de overige leden Prof. dr. I.M.C.M. Rietjens, Prof. dr. C.T.A. Evelo, Prof. dr. A.H. Piersma en Prof. dr. G.T.J. van der Horst bedanken.

Natuurlijk moet ik ook mijn collega's bij als eerste Gezondheids Risico Analyse en Toxicologie en later Toxicogenomics bedanken voor de ontzettende fijne en gezellige werksfeer! Met name de fijne samenwerkingen, leuke uitstapjes en activiteiten georganiseerd door het T.G.X. animatie team hebben dit mogelijk gemaakt!

Allereerst wil ik graag de mensen bedanken die het met mij op de kamer hebben volgehouden. Joost, Dennie en Marieke met jullie ben ik begonnen bij GRAT. Wat hebben we een lol gehad in deze eerste periode van mijn promotieonderzoek. Met name de eigenaardige attributen die Dennie in de loop der jaren had verzameld hielden de gemoederen flink bezig. Zo hing er in het begin een gigantische opblaasbare uil in het midden van onze kamer en vlogen er geregeld de nodige elastiekjes door de kamer. Af en toe vielen daar ook slachtoffers bij. Al gauw moest Joost plaats maken voor Lize. Wat hebben we gelachen om de toch wel grote verschillen tussen de Nederlandse en de Vlaamse taal. De daarop volgende jaren heb ik nog met een groot aantal mensen op de kamer gezeten die ik ook uiteraard wil bedanken voor hun collegialiteit en gezelligheid. Bedankt hiervoor/ Thanks for this Jian, Penny, Karen, Julian, Almudena, Heloise, Wim en Peter!

Uiteraard wil ook in het bijzonder de technische staf bedanken die het mede mogelijk hebben gemaakt dat er nu een mooi proefschrift ligt met de nodige resultaten. Als eerste wil ik graag Sandra bedanken voor haar hulp en expertise bij een groot aantal van mijn experimenten en met name die met de humane hepatocyten. Het was niet altijd even makkelijk, maar met jouw kennis van zaken en de nodige volharding zijn we er toch nog in geslaagd om een mooi resultaat neer te zetten. Ontzettend bedankt voor je hulp bij de kweek van de cellen maar ook bij de hele methylatie pipeline. Zonder jouw hulp was dit nooit mogelijk geweest! Bedankt hiervoor! Ook wil ik graag Karen bedanken voor met name haar hulp bij de vele ondernomen pogingen om de Exiqon analyse pipeline op te zetten in Maastricht en ook bij de $\gamma \mathrm{H} 2 \mathrm{AX}$ kleuringen. Helaas waren we niet staat om de Exiqon arrays succesvol te draaien in Maastricht maar we hebben in ieder geval een hoop geleerd over wat allemaal fout kan gaan. Ook dit is uiteindelijk allemaal goed gekomen en daarom wil ik je in ieder geval bedanken voor je kennis en hulp hierbij! Ook wil ik graag Marcel bedanken voor het draaien van de nodige Agilent en Affymetrix arrays.

Nadat alle experimenten in het lab waren afgerond, moest de data nog uitgebreid geanalyseerd worden. Dit was nooit gelukt zonder de hulp van Charly, Stan, Maarten, Daniel en Danyel. Bedankt voor jullie hulp bij de analyses en jullie geduld om alles in begrijpelijke taal uit te leggen!

Uiteraard waren er ook nog een hoop andere mede AIOs die ik graag wil bedanken voor de leuke tijd! Daarom ook Nicole, Marlon, Kimberly, Marten, Terezinha, Jarno, Marcha, Eveline, Anke, Christina, Kevin, Hilda en Joyce bedankt! Naast de AIOs wil ik ook graag de post-docs en de andere onderzoekers bedanken voor de fijne tijd bij TGX zoals Maria, Ariane, Leen, Florian, Rachel, Jan, Diana, Twan, Jacco, Theo, Tilman en Harm-Jan. 
Naast mijn wetenschappelijke collega's wil ik ook graag de mensen van het management team bedanken voor alle administratieve en financiele hulp tijdens mijn promotietraject. Dank gaat hierbij uit naar Rob, Rene, Christa, Anneloes en Sandrien. Anneloes bedankt voor al je hulp gedurende jouw loopbaan bij TGX en voor je vriendschap in de periode erna. Rene bedankt voor je bijzondere opmerkzaamheid en fascinatie voor mijn veranderende haarkleur en jouw grote verhalen over bijvoorbeeld jouw vorige leven als muzikant. Christa jou wil ik graag in het bijzonder bedanken voor je hulp bij het schrijven van de nodige brieven die voor het afronden van het promotietraject vereist waren.

Naast de mensen van de Universiteit Maastricht willen ik ook graag de onderzoekers bedanken die samen met mij aan het NTC project hebben gewerkt. In het bijzonder wil ik graag de mensen van WP1 bedanken voor de levendige discussies en opbouwende kritiek bij de velen meetings die hebben plaatsgevonden binnen deze periode. Dank gaat ook uit naar Prof. dr. O. Bekers en zijn naaste collega's van het Centraal Diagnostisch Laboratorium van het MUMC+ voor het analyseren van de samples voor de primaire humane hepatocyt studies.

Aangezien mijn promotietraject wat langer duurde als normaal wil ik ook graag de mensen van mijn nieuwe werk, als post-doc onderzoeker bij de vakgroep van Bioinformatica/BiGCaT, bedanken voor hun interesse in mijn promotieonderzoek. In het bijzonder wil ik Dr. Egon Willighagen en Prof. dr. Chris Evelo bedanken dat zij mij de mogelijkheid hebben gegeven om mijn promotietraject in een relatief kort tijdbestek succesvol af te ronden. Ook wil ik mijn collega's Susan, Martina, Freddie, Nuno, Jonathan, Ryan, Bart, Anwesha, Elisa, Ingrid en Lars bedanken voor de afwisselende pauzes en het gezellige dagje uit wat we recent ondernomen hebben!

Buiten de universiteit om wil ik ook graag een aantal mensen bedanken die, de afgelopen jaren, voor de nodige afwisseling in de vorm van gezellige avonden en uitstapjes, tijdens de tijd dat ik nu in Maastricht woon, hebben gezorgd.

Ellen, wat ontzettend leuk dat we elkaar na onze studie in Wageningen weer in Maastricht zijn tegengekomen. Ik vond het ook echt een eer om bij zowel jouw $\mathrm{PhD}$ defense als jouw bruiloft aanwezig te mogen zijn!

Marije, wat hebben we een leuke tijd gehad als huisgenoten in ons "horror" huis aan de Haspengouw. Bedankt voor jouw vriendschap en de leuke avondjes stappen in het Maastrichtse uitgaansleven!

In het bijzonder wil ik julie, Ellen en Marije, bedanken voor het feit dat jullie mijn paranimfen willen zijn tijdens mijn verdediging. Ik vind het ontzettend fijn dat jullie mij in deze spannende tijd willen bijstaan!

Ook wil ik graag Anneloes en Zoe bedanken voor hun vriendschap en de gezellige avondjes in Maastricht. Ik hoop daar binnenkort ook zeker meer tijd voor te kunnen hebben!

Naast mijn vrienden in Maastricht wil ik ook graag wat mensen bedanken in het verre Hoevelaken, Amersfoort en Utrecht. Marjolein en Annika, bedankt voor de gezellige etentjes en uitstapjes die we de afgelopen jaren hebben gemaakt. Ik vind het erg fijn dat, ondanks de afstand, we toch nog altijd vriendinnen zijn gebleven! Ook wil ik Tineke, Dietrix en Rianne bedanken voor de gezelligheid van de afgelopen jaren! Ondanks dat we elkaar niet zo heel vaak 
zien, is het toch altijd erg gezellig met jullie. Graag wil ik jullie bedanken voor de leuke film avondjes en etentjes en jullie luisterende oor in moeilijke tijden!

In de tijd dat ik nu in Maastricht woon heb ik er ook een hele nieuwe schoonfamilie bijgekregen. Graag wil ik Margo, Herman en Myrna bedanken voor de gezellige avonden, verjaardagen en feestjes van de afgelopen drie jaar. Ik vond onze bezoekjes in Parijs ook altijd erg leuk Myrna! Leon, Git en Marc ook wil ik jullie bedanken voor jullie interesse in mijn werk en bijvoorbeeld ons leuke uitstapje naar Disneyland Parijs!

Naast mijn schoonfamilie wil ik ook graag mijn eigen familie bedanken voor hun steun en interesse in mijn werk en hun bemoedigende woorden als het wat minder ging. Bedankt lieve tante Anneke en ome Gerrit. Natuurlijk hoort de verre familie in Frankrijk/Zwitserland daar ook bij! Daarnaast wil ik ook graag mijn broer Mark en zijn vriendin Angelique bedanken voor de gezelligheid en interesse in mijn werk van de afgelopen jaren. Ook al wonen we een stukje van elkaar vandaan. Ook wil mijn lieve ouders bedanken, Ria en Eef, voor hun onvoorwaardelijke steun en vertrouwen in alles wat er de afgelopen jaren is gebeurd. Bedankt dat jullie mij de kans hebben gegeven om verder te studeren, eerst in Wageningen, toen een tijdje in Newcastle en uiteindelijk in Maastricht. Bedankt ook voor de vele bezoekjes aan het mooie zuiden de afgelopen jaren.

Kevin, lieverd, eindelijk is het dan toch echt af. Buiten het feit dat er nu een mooi boekje voor mij ligt heb ik ook jou overgehouden aan mijn tijd hier in Maastricht. Zonder jouw steun en toeverlaat was het mij nooit gelukt om dit succesvol af te ronden. Bedankt dat je het de laatste drie jaar met mij hebt uitgehouden en ook wil ik je graag bedanken voor je bemoedigende woorden als het weer eens wat minder ging. Ik denk dat het niet altijd even makkelijk was om met iemand te moeten samenwonen die bezig was met promoveren. Bedankt ook dat je er altijd voor me was en voor de nodige reizen die we de afgelopen jaren hebben gemaakt. Bovendien wil ik je graag bedanken voor je vakkundigheid bij het maken van de Nederlandstalige samenvatting. Vanaf nu zal er meer tijd zijn om samen door te brengen en leuke reisjes te maken! Dankjewel lieverd! 
C

0

0

0

0

0

${ }^{\circ}$ Addendum IV

0

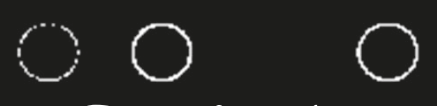

O

Curriculum Vitag

$O C$

C)

0

O

0

00

O

()

O

()

0

()

0

0

c

0

0

00

00

()

000

0

0

()

0 


\section{Curriculum Vitae}

Linda Rieswijk was born on January $7^{\text {th }} 1986$ in Amsterdam, the Netherlands. After finishing her pre-university education in the discipline "Nature and Health" at the Meridiaan College Het Nieuwe Eemland in Amersfoort, she started her bachelor study "Nutrition and Health" in 2004 at the Wageningen University. During her studies she chose the specialization of Molecular Nutrition and wrote her bachelor thesis on "Guthormones and the metabolic syndrome".

After her graduation in 2007, she continued her

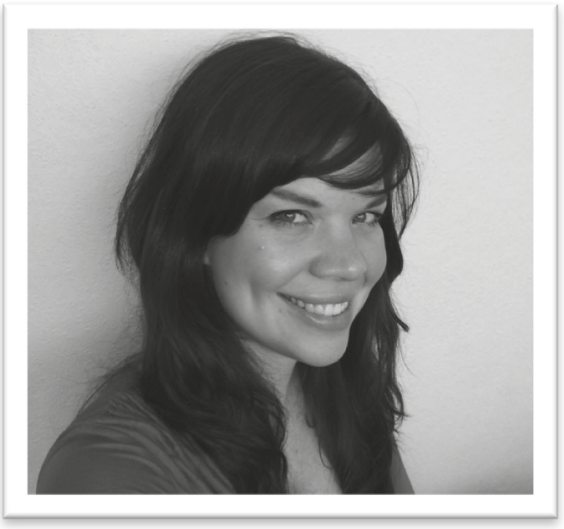
studies with a master in the same field and obtained her degree in September 2009. During her master she did a thesis for 6 months on "Antioxidative characteristics of selenium in peripheral blood mononuclear cells (PBMCs)" within the framework of the Selenium and Prostate cancer (SePros) study.

After she finished her master thesis, she went to the United Kingdom for 4.5 months to do her internship at the Human Nutrition Research Centre of the Newcastle University. Her internship covered the study of "The tissue specific effect of maternal folate depletion on the epigenetic gene regulation in mice offspring”.

On the $1^{\text {st }}$ of January 2010 she started working as a $\mathrm{PhD}$ student in the area of toxicogenomics at the Department of Health Risk Analysis and Toxicology, now also known as the Department of Toxicogenomics, of Maastricht University within a major EU financed research program of the Netherlands Toxicogenomics Centre entitled: "An applied system biology approach to predict chemical safety". Under supervision of Prof. Dr. J. Kleinjans, Dr. J. van Delft, Dr. D. Lizarraga and Dr. S. van Breda she performed the research presented in this thesis entitled: "MicroRNAs and epigenetics in chemical carcinogenesis - An integrative toxicogenomics-based approach." On the 1 ${ }^{\text {st }}$ of February 2015 she started working as a postdoctoral fellow on the eNanoMapper project within the Department of Bioinformatics (BiGCaT) at Maastricht University. Within this project she mainly focusses on the development of comprehensive ontologies for the nanosafety domain. 
C

00

0

0

0

Afddendum V

0

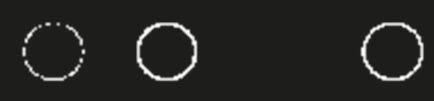

O

C) $O$

List of publications

00

O

O

O

00

O

O

0

0

()

0

0

$c$

0

0

00

00

(3)

000

O

0

0 


\section{$\underline{\text { List of publications }}$}

\section{Full research papers}

Rieswijk, L., Lizarraga, D., Brauers, K.J.J., Kleinjans, J.C.S. and van Delft, J.H.M. (2014) Characterisation of cisplatin-induced transcriptomics responses in primary mouse hepatocytes, HepG2 cells and mouse embryonic stem cells shows conservation of regulating transcription factor networks. Mutagenesis, 29 (1), 17-26.

Rieswijk, L., Brauers, K.J.J., Coonen, M.L.J., van Breda, S.G.J., Jennen, D.G.J., Kleinjans, J.C.S. (2015) Evaluating microRNA profiles reveals discriminative responses following genotoxic or non-genotoxic carcinogen exposure in primary mouse hepatocytes. Mutagenesis.

Rieswijk, L., Brauers, K.J.J., Coonen, M.L.J., van Breda, S.G.J., Jennen, D.G.J., Kleinjans, J.C.S. Improving the predictability of in vivo genotoxicity and carcinogenicity by exploiting microRNA and mRNA profiles generated in vitro from exposed primary mouse hepatocytes. In preparation

Rieswijk, L., Claessen, S.M.H., Bekers, O., van Herwijnen, M., Theunissen, D.H.J., Jennen, D.G.J., de Kok, T.M.C.M., Kleinjans, J.C.S., van Breda, S.G.J. Aflatoxin B1 exposure induces epigenetic mechanisms in primary human hepatocytes revealing novel biological processes associated with hepatocellular carcinoma. Submitted

Rieswijk, L., Claessen, S.M.H., Bekers, O., van Herwijnen, M., Theunissen, D.H.J., Jennen, D.G.J., de Kok, T.M.C.M., Kleinjans, J.C.S., van Breda, S.G.J. Persistent microRNA-gene networks in primary human hepatocytes upon withdrawal of aflatoxin B1 exposure are related to hepatocellular carcinoma. Submitted

Rieswijk, L., Claessen, S.M.H., Bekers, O., van Herwijnen, M., Theunissen, D.H.J., Jennen, D.G.J., de Kok, T.M.C.M., Kleinjans, J.C.S., van Breda, S.G.J. Aflatoxin B1 induces persistent epigenomic effects in primary human hepatocytes associated with hepatocellular carcinoma. Submitted

\section{Abstracts}

Rieswijk, L.; Lizárraga, D.; Brauers, K.J.J.; Gaj, S.; Kleinjans, J.C.S. and van Delft, J.H.M. Predicting in vivo genotoxicity using mRNA and miRNA data from two in vitro liver models. Poster presentation at GROW Science Day, November 2013, Maastricht, The Netherlands.

Rieswijk, L.; Lizárraga, D.; Brauers, K.J.J.; Gaj, S.; van Delft, J.H.M. and Kleinjans, J.C.S. Predicting in vivo genotoxicity using mRNA and miRNA data from two in vitro liver models. Poster presentation at $49^{\text {th }}$ Congress of the European Societies of Toxicology (EUROTOX), September 2013, Interlaken, Switzerland.

Rieswijk, L.; Lizárraga, D.; Brauers, K.J.J.; Gaj, S.; Kleinjans, J.C.S. and van Delft, J.H.M. miRNA-mRNA-TF interaction networks in response to various toxic agents using primary mouse hepatocytes. Oral presentation at the Annual Meeting/PhD days of the Dutch Society of Toxicology, June 2013, Zeist, The Netherlands. 
Rieswijk, L.; Lizárraga, D.; Brauers, K.J.J.; Gaj, S.; Kleinjans, J.C.S. and van Delft, J.H.M. Identify mechanisms of toxicity for true genotoxins, non-genotoxins and false positive genotoxins by the integration of microRNA and mRNA profiles using integrative pathway analysis in HepG2 cells and primary mouse hepatocytes. Oral presentation at the 4th Annual meeting of the Netherlands Toxicogenomics Centre, January 2013, Amsterdam, The Netherlands.

Rieswijk, L.; Lizárraga, D.; Brauers, K.J.J.; Gaj, S.; Kleinjans, J.C.S. and van Delft, J.H.M. Identify mechanisms of toxicity for true genotoxins, non-genotoxins and false positive genotoxins by the integration of microRNA and mRNA profiles using integrative pathway analysis in HepG2 cells and primary mouse hepatocytes. Oral presentation at the Grow Science Day, November 2012, Maastricht, The Netherlands.

Rieswijk, L.; Lizárraga, D.; Brauers, K.J.J.; Gaj, S.; Kleinjans, J.C.S. and van Delft, J.H.M. Identify mechanisms of toxicity for true genotoxins, non-genotoxins and false positive genotoxins by the integration of microRNA and mRNA profiles using integrative pathway analysis in HepG2 cells and primary mouse hepatocytes. Poster presentation at the 42nd European Environmental Mutagen Society, September 2012, Warsaw, Poland.

Rieswijk, L.; Lizárraga, D.; Brauers, K.J.J.; Gaj, S.; van Delft, J.H.M. and Kleinjans, J.C.S. Identify common mechanisms of toxicity for true genotoxins, non-genotoxins and false positive-genotoxins in HepG2 cells by the integration of mRNA and miRNA profiles. Poster presentation at the Annual Meeting/PhD days of the Dutch Society of Toxicology, June 2012, Zeist, The Netherlands.

Rieswijk, L.; Lizárraga, D.; Brauers, K.J.J.; Gaj, S.; van de Water, Bob; Vrieling, Harry; Kleinjans, J.C.S. and van Delft, J.H.M. Characterization of the conserved CDDP-induced transcriptomic-based DNA damage response in primary mouse hepatocytes, HepG2 cells and mouse embryonic stem cells. Poster presentation at the Annual Meeting/PhD days for the Dutch Society of Toxicology, June 2011, Zeist, The Netherlands.

Rieswijk, L.; Lizárraga, D.; Brauers, K.J.J.; Gaj, S.; van de Water, B.; Vrieling, H.; Kleinjans, J.C.S. and van Delft, J.H.M. Cisplatin-induced genotoxic stress in primary mouse hepatocytes, mouse embryonic stem cells and HepG2 cells; based on mRNA and microRNA expression profiles. Poster presentation at the $50^{\text {th }}$ Annual Meeting of the Society of Toxicology, March 2011,Washington D.C., United States.

Rieswijk, L.; Lizárraga, D.; Kleinjans, J. C.S. and van Delft, J.H.M. The role of microRNA and epigenetics in DNA damage responses. Poster presentation at the Annual Meeting/PhD days of the Dutch Society of Toxicology, June 2011, Zeist, The Netherlands.

\section{Patents}

In Vitro Method For Predicting Whether A Compound Is Genotoxic In Vivo (Europe 213 EPP0)

Inventors: Daneida Lizarraga Lopez, Linda Rieswijk, Joseph H.M. van Delft and Joseph C.S. Kleinjans 


\section{Achievements}

Travel grant awarded by the Dutch Society of Toxicology for attending the 42nd European Environmental Mutagen Society, September 2012, Warsaw, Poland.

Price for best poster during Annual Meeting/PhD days of the Dutch Society of Toxicology, June 2012, Zeist, The Netherlands. 